\title{
Model Reference Adaptive Control of Piecewise Linear Systems with Applications to Aircraft Flight Control
}

\author{
A Dissertation \\ Presented to \\ the faculty of the School of Engineering and Applied Science \\ University of Virginia
}

In Partial Fulfillment

of the requirements for the Degree

Doctor of Philosophy (Electrical Engineering)

by

QIAN SANG

May 2012 


\section{APPROVAL SHEET}

The dissertation is submitted in partial fulfillment of the requirements for the degree of

Doctor of Philosophy (Electrical Engineering)

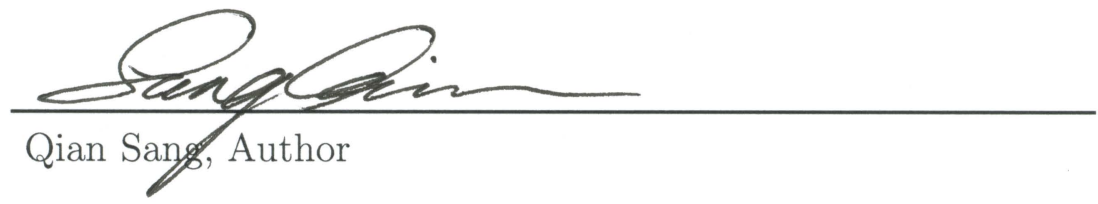

This dissertation has been read and approved by the examining committee:
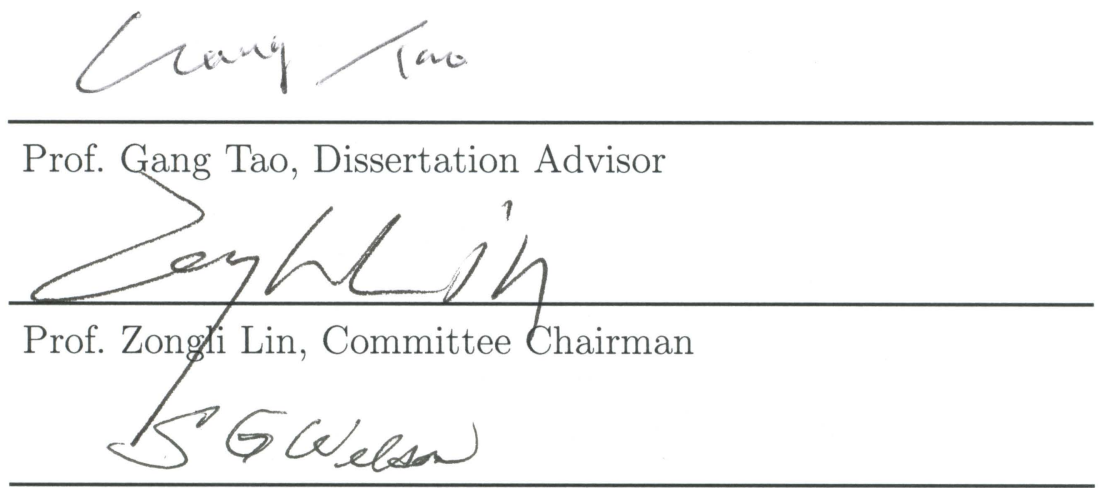

Prof. Stephen G. Wilson

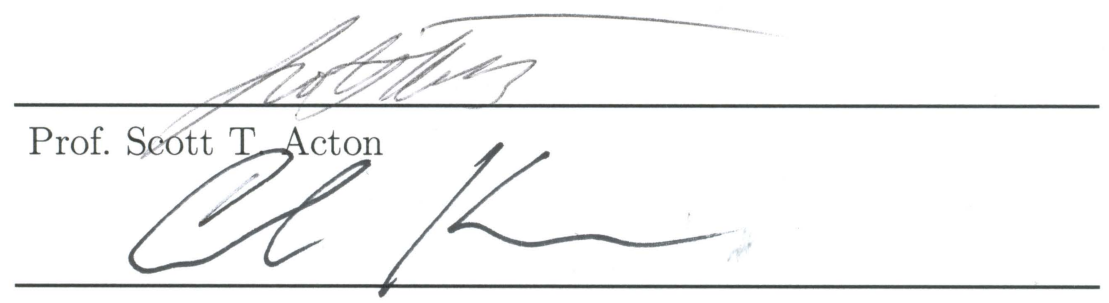

Prof. Carl R. Knospe, Mechanical and Aerospace Engineering

Accepted for the School of Engineering and Applied Science:
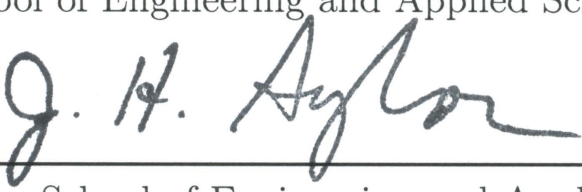

Dean, School of Engineering and Applied Science

May, 2012 


\begin{abstract}
As an effort to establish a stability and performance metric for adaptive control systems and an attempt to expand nonlinear system operating range with linearizationbased designs in the presence of uncertainties, this dissertation focuses on the gain margin (GM) of adaptive control systems and the development of novel adaptive control schemes for piecewise linear systems. The contributions are a systematic gain margin analysis for a variety of adaptive control systems, and a framework of solutions to the open problems in adaptive control of uncertain piecewise linear systems.

The gain margin of adaptive control systems is defined as the specification of the parameter range of a control gain matrix in a designed adaptive control system for maintaining the desired closed-loop signal boundedness and asymptotic tracking performance. A systematic gain margin analysis is conducted for continuous- and discrete-time, direct and indirect model reference adaptive control (MRAC) systems, adaptive state feedback control systems, and sampled-data adaptive control systems. The derived gain margin results are applicable to systems with adaptive nonlinear or pole placement control designs. Methods for enlarging the GM by proper choices of design parameters are presented. This gain margin study has established guidelines for designing control systems with stability and tracking guarantees in the presence of uncertain control gain variations. Furthermore, the derived gain margin results are applied to solve the problem of system performance robustness with respect to reduced actuator effectiveness.

The adaptive control approach to piecewise linear systems is largely unexploited, despite the tremendous growth of research interest in stability analysis and control design for such systems over the past two decades. This dissertation focuses on the development of model reference adaptive control designs for piecewise linear systems to
\end{abstract}


achieve closed-loop stability (signal boundedness) and system state or output tracking as close as possible, in the presence of structural and parametric uncertainties and repetitive system mode switches. State feedback for state tracking (SFST), state feedback for output tracking (SFOT), and output feedback for output tracking (OFOT) MRAC designs are presented. It is shown that under a slow system mode switching condition, closed-loop stability and a small tracking error in the mean-square sense are achieved. For the SFST design, asymptotic state tracking performance is restored under certain persistency of excitation (P.E.) condition, and the slow switching condition can be relaxed to arbitrary system mode switches, when a common Lyapunov function exists for the constituent system modes. For the SFOT design, asymptotic tracking performance is ensured for arbitrary system mode switches, in addition to closed-loop stability, under an additional plant-model matching condition. Effectiveness of the proposed adaptive control schemes are demonstrated by simulation studies on linearized and nonlinear aircraft models (NASA generic transport model (GTM)). 


\section{Acknowledgments}

I would like to express my deepest and sincerest gratitude and appreciation to my advisor, Professor Gang Tao, for his guidance, persistent encouragement, and continuous support throughout my Ph.D. study that has led to this dissertation. Professor Tao introduced me to this brand new field of adaptive control. He has been always readily available for research discussions and has guided me through the challenges of my research. He has set an example to me with his professionalism, the breadth and depth of his knowledge, and his impeccable attitude towards research.

I am grateful to Dr. Suresh M. Joshi, NASA Langley Research Center, for his helpful motivation, comments, and suggestions on my research work.

I would also like to thank Professor Zongli Lin, Professor Stephen G. Wilson, Professor Scott T. Acton, and Professor Carl R. Knospe for serving on my Ph.D. advisory committee.

It is a blessing to be surrounded by many colleagues and friends at the University of Virginia. I am particularly grateful to Jiaxing Guo, Yu Liu, Xinmin Liu, Simon Mushi, Houbing Song, Parinya Anantachaisilp for their stimulating discussions and help, and for making my life at U.Va. enjoyable.

I gratefully acknowledge the financial support provided by NSF under grant ECS0601475 and by NASA Langley Research Center under grant NNX08AB99A.

I want to thank my wife Ran Zheng, my son Brandon, my parents and my parentsin-law, without whom this dissertation would not have been possible and meaningful. This dissertation is dedicated to my family.

QIAN SANG

Charlottesville, Virginia 


\section{Contents}

1 Introduction 2

1.1 Research Motivation ................... 4

1.2 Literature Overview . . . . . . . . . . . . . . . . . 7

1.3 Dissertation Outline . . . . . . . . . . . . . . . . . . 10

2 Basic Problem Formulation and Background 13

2.1 Basic Problem Formulation . . . . . . . . . . . . . . 13

2.1.1 Gain Margins of Adaptive Control Systems . . . . . . . . . . . 13

2.1.2 Performance Robustness of MRAC with respect to Reduced Actuator Effectiveness . . . . . . . . . . . . . 15

2.1.3 Adaptive Control of Piecewise Linear Systems . . . . . . . . . 16

2.2 Background . . . . . . . . . . . . . . . . . 19

2.2.1 Adaptive Control Methodology . . . . . . . . . . . . . 19

2.2.2 LDU and LDS Decompositions of a Gain Matrix $K_{p}$. . . . . 23

3 Gain Margins of Adaptive Control Systems 24

3.1 Problem Statement . . . . . . . . . . . . . . 25

3.2 LDU Decomposition of $K_{p} K \ldots \ldots \ldots \ldots$. . . . . . . . 28

3.3 Gain Margins of Continuous-Time MRAC Systems . . . . . . . . . 30

3.3.1 Model Reference Control System . . . . . . . . . . . . . . . . 31 
3.3 .2 Design for SISO Systems . . . . . . . . . . . . . . . . . . 33

3.3.3 LDS Decomposition Based Design for MIMO Systems . . . . 36

3.3.4 Extensions to Other Adaptive Control Systems _ . . . . . . . 39

3.4 Gain Margins of Discrete-Time MRAC Systems . . . . . . . . . 42

3.4.1 Design for SISO Systems . . . . . . . . . . . . . . . . 42

3.4.2 LDS Decomposition Based Design for MIMO Systems . . . . . 45

3.4.3 Gain Margin Bounds for $M=2 \ldots \ldots \ldots \ldots$

3.5 Gain Margins of Indirect MRAC Systems . . . . . . . . . . . . 51

3.5.1 Design for Continuous-Time SISO Systems . . . . . . . . . 51

3.5.2 Design for Discrete-Time SISO Systems . . . . . . . . . . . 54

3.5.3 Design for A Class of Multivariable Systems . . . . . . . . 56

3.6 Gain Margins of Sampled-Data Adaptive Control Systems _ . . . . . 61

3.6.1 MRAC of Sampled-Data Systems . . . . . . . . . . . 61

3.6 .2 Gain Margin Analysis . . . . . . . . . . . . . . . 62

3.7 Simulation Study . . . . . . . . . . . . . . . . . . . . 65

4 Performance Robustness of MRAC with respect to Reduced Actu$\begin{array}{ll}\text { ator Effectiveness } & \mathbf{7 0}\end{array}$

4.1 Performance Robustness Issue for MRAC with respect to Reduced Actuator Effectiveness . . . . . . . . . . . . . . . 71

4.2 Continuous-Time MIMO Direct MRAC . . . . . . . . . . . 73

4.3 Discrete-Time MIMO Direct MRAC . . . . . . . . . . . 74

4.3.1 Performance Robustness of the LDS Based Design . . . . . . 75

4.3.2 Performance Robustness of the LDU Based Design _ . . . . 78

4.4 Indirect MRAC Designs $\ldots \ldots \ldots \ldots \ldots$

4.5 Simulation Study . . . . . . . . . . . . . . . . . . . 82 
5 Adaptive Control of Piecewise Linear Systems with State Feedback for State Tracking $\quad 86$

5.1 Problem Statement . . . . . . . . . . . . . . . . . . . . . . . 87

5.2 Time-Varying Reference Model Systems _ . . . . . . . . . . . 88

5.2.1 Piecewise Linear Reference Model System . . . . . . . . . 88

5.2.2 Stability of Piecewise Linear Systems _ . . . . . . . . . . 88

5.3 Design for Single-Input Systems . . . . . . . . . . . . . . 90

5.3 .1 Controller Structure _. . . . . . . . . . . . . . . . . . 90

5.3 .2 Adaptive Laws . . . . . . . . . . . . . . . . . . . . . . . 91

5.3 .3 Stability and Tracking Properties _. . . . . . . . . . 92

5.3.4 Adaptation with Sufficiently Rich Reference Input . . . . . . . 95

5.3.5 Simulation Study . . . . . . . . . . . . . . . . . . 96

5.4 Design for Multiple-Input Systems . . . . . . . . . . . . . . . 97

5.4.1 Reference Model System . . . . . . . . . . . . . . . . . . . 99

5.4 .2 Controller Structure _. . . . . . . . . . . . . . . . 100

5.4 .3 Adaptive Laws . . . . . . . . . . . . . . . . . . . . . 101

5.4.4 Simulation on NASA GTM _ . . . . . . . . . . . 105

5.5 Extensions to State-Dependent Switches _. . . . . . . . . . . 109

5.5.1 Problem Statement . . . . . . . . . . . . . . . . . . 113

5.5 .2 Adaptive Control Design . . . . . . . . . . . . . . . . . 114

6 Adaptive Control of Piecewise Linear Systems with State Feedback for Output Tracking $\quad 122$

6.1 Design for SISO Piecewise Linear Systems _ . . . . . . . . . . 123

6.1.1 Problem Statement . . . . . . . . . . . . . . . . 123

6.1.2 Nominal Control Scheme . . . . . . . . . . . . . . . 125 
6.1 .3 Adaptive Control Design . . . . . . . . . . . . . . . . . . . 129

6.1.4 Recovery of Asymptotic Tracking Performance . . . . . . . . . 134

6.1.5 Advantages over Conventional MRAC . . . . . . . . . 135

6.2 Design for MIMO Piecewise Linear Systems . . . . . . . . . . 137

6.2 .1 Problem Statement . . . . . . . . . . . . . . . . . . . 137

6.2 .2 Nominal Control Scheme . . . . . . . . . . . . . . . . . . 140

6.2.3 Adaptive Control Scheme . . . . . . . . . . . . . . . . . . 142

6.2.4 Recovery of Asymptotic Tracking Performance . . . . . . . . 147

6.2.5 Advantages over Conventional MRAC . . . . . . . . . . . . 149

6.3 Simulation Study . . . . . . . . . . . . . . . . . . . . . 151

6.3.1 SISO Piecewise Linear Systems in CCF . . . . . . . . . . . 152

6.3.2 Linearized Lateral-Directional NASA GTM Model . . . . . . . 154

7 Adaptive Control of Piecewise Linear Systems with Output Feedback for Output Tracking $\quad 162$

7.1 Problem Statement . . . . . . . . . . . . . . . . . . . . . . 163

7.2 Nominal Control Design _ . . . . . . . . . . . . . . . . 166

7.2.1 Controller Structure _. . . . . . . . . . . . . . 166

$7.2 .2 \quad$ Stability Properties . . . . . . . . . . . . . 168

7.3 Adaptive Control Design . . . . . . . . . . . . . . . . . . . 170

7.3.1 Controller Structure . . . . . . . . . . . . . . . . 170

7.3 .2 Adaptive Law Design . . . . . . . . . . . . . . . . . . . 172

7.3.3 Stability Properties . . . . . . . . . . . . . . . . . . 174

7.4 Advantages over Conventional MRAC . . . . . . . . . . . 176

7.5 Simulation Study . . . . . . . . . . . . . . . 178 
8 Conclusions $\quad 184$

9 Appendix 188

9.1 Derivations of $(3.4 .15)-(3.4 .18) \ldots \ldots \ldots \ldots$

9.2 Proof of Lemma $5.2 .1 \ldots \ldots \ldots$. . . . . . . . . . . . . . 190

9.3 Proof of Theorem 5.3.1 . . . . . . . . . . . . . . . . . . . 192

9.4 Proof of Theorem 5.3.5 . . . . . . . . . . . . . . . . . . 194

$\begin{array}{ll}\text { References } & 198\end{array}$ 


\section{List of Figures}

2.1 Adaptive control system with a gain matrix $K \ldots \ldots \ldots \ldots$

3.1 Adaptive control system with a gain matrix $K \ldots \ldots \ldots \ldots$

3.2 High frequency gain $k_{p}$ and GM upper bound $k^{0}$ versus T. . . . . 64

3.3 Output tracking error $\boldsymbol{e}(t)=\left[e_{1}(t), e_{2}(t)\right]^{\top}$ for $k_{1}=0.9 \in(0,1.2]$ and

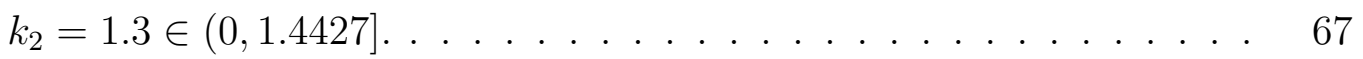

3.4 Output tracking error $\boldsymbol{e}(t)=\left[e_{1}(t), e_{2}(t)\right]^{\top}$ for $k_{1}=0.9 \in(0,1.2]$ and $k_{2}=1.8 \notin(0,1.4427] \ldots \ldots \ldots \ldots \ldots \ldots \ldots \ldots \ldots \ldots$

3.5 Output tracking error $\boldsymbol{e}(t)=\left[e_{1}(t), e_{2}(t)\right]^{\top}$ for $k_{1}=0.9 \in(0,1.2]$ and

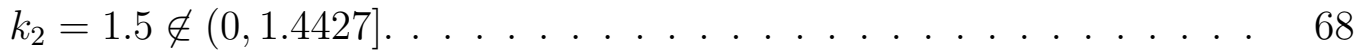

4.1 Adaptive control system with reduction in actuator effectiveness. . . . 72

4.2 Tracking error $e(t)$ for $K=\operatorname{diag}\{0.4,0.6\} \ldots \ldots \ldots \ldots$

4.3 Tracking error $e(t)$ for $K=\operatorname{diag}\{0.1,1.0\} \ldots \ldots \ldots \ldots$

4.4 Tracking error $e(t)$ for $K=\operatorname{diag}\{0.25,0.65\} \ldots \ldots \ldots \ldots$

5.1 State tracking error $\boldsymbol{e}(t)$ for $r(t)=2 \sin (0.01 \pi t)$ and $T_{0}=1000 \mathrm{~s} \ldots \ldots 97$

5.2 State tracking error $\boldsymbol{e}(t)$ for $r(t)=2 \sin (0.01 \pi t)+2 \sin (0.02 \pi t)+$ $2 \sin (0.005 \pi t)$ (sufficiently rich) and $T_{0}=1000 \mathrm{~s} \ldots \ldots \ldots 98$

5.3 Simulations for linear system: state tracking error $\boldsymbol{e}(t)$ for the proposed control scheme (above) and for the 60\% fixed control law (below). . . 110 
5.4 GTM simulation: $\boldsymbol{e}(t)$ for the proposed control scheme in Section 5.4.3.1 (above) and for the 60\% fixed control law (below). . . . . . . . 111

5.5 GTM simulation: control input $\boldsymbol{u}(t)=\left[\delta_{e}, \delta_{T}\right]^{\top}$ for the control scheme in Section 5.4.3.1 (above) and for the 60\% fixed control law (below). . 112

5.6 Mass-spring-damper system with a one-sided spring. . . . . . . . . . . 119

5.7 State tracking error $e(t)$ for Simulation I: the LTI reference model system in (5.5.15). . . . . . . . . . . . . . . . . . 121

5.8 State tracking error $e(t)$ for Simulation II: the piecewise linear reference model system (5.5.2) with parameter matrices as in (5.5.16). . . . . . 121

6.1 Tracking error $e(t)$ with $r(t)=2$ : the proposed scheme (top) vs. the conventional MRAC scheme (bottom). . . . . . . . . . . . . . 153

6.2 Tracking error $e(t)$ with $r(t)=2 \sin (0.5 t)$ : the proposed scheme (top) vs. the conventional MRAC scheme (bottom). . . . . . . . . . . . . 154

6.3 Simulation I: tracking performance of the proposed scheme. . . . . . 157

6.4 Simulation I: control input $\boldsymbol{u}(t)$ of the proposed scheme. . . . . . . 158

6.5 Simulation I: tracking performance of the conventional MRAC scheme. 158

6.6 Simulation I: control input $\boldsymbol{u}(t)$ of the conventional MRAC scheme. . 159

6.7 Simulation II: tracking performance of the proposed scheme. . . . . . 159

6.8 Simulation II: control input $\boldsymbol{u}(t)$ of the proposed scheme. . . . . . . . 160

6.9 Simulation II: tracking performance of the conventional MRAC scheme. 160

6.10 Simulation II: control input $\boldsymbol{u}(t)$ of the conventional MRAC scheme. . 161

7.1 Output tracking performance and the control input $\delta_{e}$ with $r(t)=$ $0.2 \sin (\pi t / 200)$ : the proposed scheme. . . . . . . . . . . . . . 181

7.2 Output tracking performance and the control input $\delta_{e}$ with $r(t)=$ $0.2 \sin (\pi t / 200)$ : the conventional MRAC scheme. . . . . . . . . . . . 181 
7.3 Output tracking performance and the control input $\delta_{e}$ with $r(t)=0.2$ : the proposed scheme. . . . . . . . . . . . . . . . . . . . . . 182

7.4 Output tracking performance and the control input $\delta_{e}$ with $r(t)=0.2$ : the conventional MRAC scheme. . . . . . . . . . . . . . . . . . . 182 


\section{Chapter 1}

\section{Introduction}

The design of control systems for performance-critical systems, such as aircraft flight control systems, to make the controlled outputs track desired trajectories despite parametric, structural, or environmental uncertainties, is of both theoretical and practical interests. Their ability to automatically adjust a controller by adaptive laws to deal with such uncertainties to achieve desired system performance makes adaptive control designs attractive for performance-critical system applications. Recently there has been considerably increased effort in research on adaptive control for aircraft flight systems in the presence of uncertainties and failures. As a main and matured approach of adaptive control, model reference adaptive control (MRAC) is capable of making the outputs of the controlled system to track the outputs of a reference model system in addition to closed-loop stability. The study of multivariable MRAC systems expands to some important new research topics including gain margin (GM) specification and adaptive control of piecewise linear systems, of interest to such applications.

The gain margin concept, originally defined for linear time-invariant (LTI) control systems to specify a necessary and sufficient range (relative to a chosen value) of a control gain for stability, is also important for adaptive control systems to charac- 
terize their relative stability and performance robustness properties with respect to control gain uncertainties. In this dissertation, the study of gain margin for MRAC systems is initialized based on their stability properties, and the research effort has been extended to a systematic investigation of the gain margins for commonly used adaptive control systems, including some popular adaptive nonlinear control designs such as those using feedback linearization or backstepping.

By a piecewise linear system (also called a "switched linear system" in the literature by many researchers), we mean a dynamical system whose dynamics switches among a set of continuous-time subsystems according to certain switching criteria. For instance, the continuous state space (e.g., $\mathbb{R}^{n}$ ) is partitioned by some hyperplanes into several operating regions, each corresponding to a subsystem describing the system dynamics when the state falls into that region, and the system dynamics switches when its state trajectory crosses the hyperplane that separates two adjacent operating regions ("state-dependent switch"); the system dynamics switches according to a certain time sequence, which may or may not be known a priori ("time-dependent switch"). There has been increased research effort in the stability analysis and control design of such systems over the past two decades (see [1,2] and the references therein). Although the stability conditions derived and control designs developed have inherent robustness to a certain extent with regard to system parameter uncertainties, precise knowledge of each constituent subsystem is usually assumed to be available, and the tracking control problem in the presence of system structural and parametric uncertainties is rarely dealt with explicitly in the literature. The goals of this dissertation are to develop novel adaptive control designs that can effectively handle large structural and parametric uncertainties and repetitive switches in piecewise linear systems, and to establish a systematic framework of the adaptive control 
approach to piecewise linear systems. The key feature of such an adaptive control approach is for piecewise linear systems to achieve desired state or output tracking performance, in addition to closed-loop stability (signal boundedness).

In the reminder of this chapter, the motivation for this research is discussed, followed by an overview of the related work in the existing literature. The organization of this dissertation is outlined at the end of the chapter.

\section{$1.1 \quad$ Research Motivation}

Adaptive control theory and its application to aircraft flight control has been a focus in the study of control systems. A major advantage of the adaptive control approach over classical control and gain-scheduling techniques is its capability of effectively accommodating large system parametric and structural uncertainties. Despite the remarkable advances in adaptive control theory and successful experience in its applications to aircraft flight control, the lack of verification and validation methods has been a major obstacle to the implementation of adaptive control on performancecritical systems. The development of a suitable, quantitative stability and performance metric for adaptive control systems motivates this study of gain margin for adaptive control systems.

Gain margin (GM) is a well accepted quantitative metric of relative stability for classical control systems, and has been extensively studied in the literature. It is defined for linear time-invariant systems as a range of a forward-loop control gain within which the closed-loop system is stable. For adaptive control systems, a suitable metric for stability and performance such as gain margin is desirable as a performance measure useful in evaluating and comparing adaptive controllers and maturing adaptive control approaches for implementation on performance-critical systems. Typical con- 
trol objectives for an adaptive control system are closed-loop stability (signal boundedness) and asymptotic tracking performance. Therefore, in the context of adaptive control and resembling its counterpart in classical linear control theory, the GM is defined as the specification of the parameter range of a control gain matrix in a designed adaptive control system for maintaining the desired asymptotic tracking performance, in addition to closed-loop signal boundedness.

A second motivation for this research on the gain margin concept of adaptive control systems lies in its applicability to the investigation of robustness properties of the system closed-loop stability and asymptotic tracking performance with respect to reduced actuator effectiveness in aircraft flight control systems. Actuators are driven by control signals to move the control surfaces (ailerons, rudders, elevators, etc.) to generate aerodynamic forces and moments on the aircraft so as to achieve the desired control objectives. Uncertain actuator failures and damages can occur, including reduction in effectiveness ("loss-of-effectiveness," in some research papers), floating, lock-in-place, and hard-over. Lack of appropriate compensation can severely deteriorate system performance, or even lead to instability and cause catastrophic accidents. Reduction in actuator effectiveness is the type of actuator failure in which partial control surface is damaged and the effectiveness reduces to an uncertain fraction of the normal level (that without reduction in effectiveness). It is modeled in the literature as an (uncertain) actuator effectiveness matrix at the system control inputs, based on which several adaptive control designs have been proposed for compensation. However, no investigation has been conducted into the inherent performance robustness properties of adaptive control systems to such actuator failures. It is clear that such a problem formulation fits in the context of the gain margin study, so that the derived gain margin results for adaptive control systems can be directly applicable 
to its solution.

The gain margin study is meaningful in establishing guidelines for designing control systems with stability and tracking guarantees in the presence of uncertain control gain variations.

The motivation to study the adaptive control problem for piecewise linear systems is two-fold. On the one hand, as will be presented in Chapter 2, a nonlinear system may be modeled as a piecewise linear system for control design, which is expected to be capable of expanding the system operating range. A common practice in control of a nonlinear system is to consider its linearized model at some operating point, within a neighborhood of which it can accurately describe the nonlinear system behavior, and a wealth of linearization-based design techniques can be applied to achieve the control objective. This linearized model is often taken to be time-invariant, and the neighborhood may be small, thus the applicability of a linearization-based design may be limited. This limitation motivates a piecewise linear system model as an approximation of the nonlinear system for control design, which consists of a set of linear time-invariant (LTI) subsystems, each being a valid model of the nonlinear system within a neighborhood of an operating point. These neighborhoods are pieced together such that the operating range of interest is covered, and transitions of operating points are modeled as "switches" between the corresponding subsystems.

On the other hand, the research motivation to study piecewise linear systems comes from the fact that many practical systems are of a hybrid nature and require several dynamical subsystems to describe their behavior [3], e.g., the motion of an automobile subject to a manual or an automatic transmission [4], biological systems and chemical processes [5], and power electronics [6].

The challenge in control of piecewise linear systems lies in the fact that prop- 
erties of each individual subsystem are not necessarily inherited by the piecewise linear system, such that traditional control theory developed for continuous-time linear time-invariant or time-varying systems may not be directly applicable to piecewise linear systems [1]. For example, it is well known that the stability of an autonomous piecewise linear system is not guaranteed under fast subsystem switches in spite of the fact that each subsystem is asymptotically stable. The control task is even more challenging in the presence of large system structural and parametric uncertainties. Therefore, it is of both theoretical and practical value to develop effective control schemes for piecewise linear systems such that desired objectives and stability properties can be ensured. For such a control problem, the adaptive control approach is a suitable solution with its capacity to effectively accommodate large system parametric and structural uncertainties.

\subsection{Literature Overview}

Relative stability, i.e., the gain margin (GM) and phase margin (PM) concepts, is an important issue and has been studied extensively for classical control systems $[7,8]$. Although the stability properties of a wide class of adaptive control systems have been studied and analyzed extensively [9-15], there is a lack of literature about stability margins for adaptive control systems. Stability margins in the existing literature are either defined for certain specific adaptive control systems, or the results are conservative as compared with those obtained from numerical simulations [16-18]. The challenge to the study of a time-delay margin (phase margin) is the lack of an analytical tool to perform such an analysis. In this dissertation, the focus is on the characterization of the relative stability of adaptive control system by gain margin, which specifies a sufficient parameter range of a control gain matrix at the 
inputs of the controlled system for maintaining closed-loop stability and asymptotic tracking performance. Such a characterization is general enough so that various MRAC systems, adaptive state feedback control systems, and nonlinear adaptive control systems using feedback linearization or backstepping designs can be studied.

The study of piecewise linear systems has attracted increasing research effort since the 1990s and the field has witnessed a tremendous growth of interest, as can been seen from the monographs $[1,2]$, the survey papers [19-24], special issues of major journals [25-28], and the references therein. It is well known that a piecewise linear system may not necessarily inherit stability properties from its constituent subsystems. For instance, fast subsystem switches can destabilize the system even if all subsystems are asymptotically stable. On the other hand, a piecewise linear system may be stabilized under certain switching sequences even if all subsystems are unstable. Based on these observations, two problems are of great interest and of particular relevance to the research task in this dissertation: (1) Under what conditions is asymptotic (or exponential) stability of the piecewise system guaranteed for arbitrary system subsystem switches? (2) What conditions are needed for ensuring asymptotic (or exponential) stability of the piecewise linear system if it cannot be guaranteed for arbitrary subsystem switches? The work in [29-37] was devoted to the former question by searching for a common Lyapunov function for the piecewise linear system, while $[19,38]$ proposed the so-called "dwell-time" switching strategy to characterize a sufficient, slow-switching condition for asymptotic stability of piecewise linear system, which was then extended to the "average dwell-time" conditions [39-44].

Considerable research effort has been devoted to developing analysis tools and control strategies for stability and stabilization of piecewise linear systems [43-54]. However, not much effort has been made in the adaptive control literature to address 
parametric and structural uncertainties in piecewise linear systems. When adaptive control schemes designed for LTI systems are applied to piecewise linear systems, in addition to degraded tracking performance, closed-loop stability may be lost for fast subsystem switches. Some modifications are made to the standard adaptive control schemes, aiming at an improved tracking performance of piecewise linear system. In [55], a switching control scheme is presented to deal with control of systems with abruptly jumping parameters, and a stability condition on the frequency of the parameter discontinuities is derived. A multiple model switching and tuning approach is considered in [56] which is effective in reducing transient tracking error of a piecewise linear system. The adaptive control scheme proposed in [57] for piecewise linear systems achieved closed-loop stability and small tracking error in the mean-square sense provided that switches occur over sufficiently large time intervals. However, for all these designs, asymptotic tracking performance cannot be achieved due to repetitive system switches. An adaptive control scheme was presented in $[58,59]$ for bimodal piecewise linear systems, but the assumption of canonical forms limits its applicability to system with more general structures.

An effective adaptive state feedback control scheme that achieves closed-loop stability and asymptotic state tracking was proposed in [60], where the reference model is chosen to be LTI. Such a choice imposes stringent structural requirements on the constituent subsystems; that is, each constituent subsystem has to match the same LTI reference model through some (unknown) nominal controller parameters, which may not be feasible in practical applications. In this dissertation (see Chapter 5), we will present a reference model system which is also piecewise linear and study the related stability problems. Such a time-varying reference model system is investigated based on the properties of piecewise linear systems $[1,19,39]$. 
While the gain margin study is initialized on MRAC systems, a systematic gain margin analysis is conducted on various adaptive control systems, direct and indirect, continuous-time and discrete-time, also including sampled-data adaptive control systems and some adaptive nonlinear control systems. In the research of the adaptive control approach to piecewise linear systems, the focus is placed on continuous-time direct MRAC designs in the presence of structural and parametric uncertainties.

\subsection{Dissertation Outline}

This dissertation establishes the gain margin concept, and conducts a thorough investigation of the gain margins for a wide variety of adaptive control systems. The results are then applied for a solution of the performance robustness problem of MRAC under reduction in actuator effectiveness. For adaptive control of piecewise linear system$\mathrm{s}$, the dissertation presents the design conditions, adaptive control designs, stability properties and tracking performance evaluations with simulation studies for effectiveness demonstration. The major results in this dissertation have been published in the following journal and conference papers: [61-63] (Chapter 3), [64] (Chapter 4), [65-68] (Chapter 5), [69-71] (Chapter 6), [72] (Chapter 7).

The dissertation is organized as follows.

In Chapter 2, the basic problems considered in this dissertation are formulated. Some background on the adaptive control methodology and multivariable adaptive control is briefly reviewed.

In Chapter 3, a systematic gain margin study is presented for many commonly used adaptive control systems, including continuous- and discrete-time direct MRAC schemes applied to multi-input, multi-output (MIMO) linear time-invariant (LTI) systems, some indirect multivariable MRAC systems, and systems with adaptive non- 
linear or pole placement control designs. Methods for enlarging the GM by proper choices of design parameters are presented. Moreover, the effect of sample time on the GM of sampled-data adaptive control systems is studied, which establishes the relationship between the continuous-time and discrete-time GM results. Simulation examples are provided to illustrate some of the theoretical results.

In Chapter 4, the problem of performance robustness of MRAC schemes with respect to reduction in actuator effectiveness (loss-of-effectiveness) is studied for direct multivariable MRAC, as well as indirect MRAC, for both continuous-time and discrete-time schemes. The reduction is modeled by an uncertain gain matrix representing the actuator effectiveness at the control inputs. MRAC systems are analyzed to determine the robustness of the desired closed-loop performance of stability and asymptotic tracking with respect to such reductions.

In Chapter 5, direct adaptive state feedback for state tracking (SFST) MRAC designs for piecewise linear systems are developed. Piecewise linear reference model systems are used for generating desired state trajectories and their stability properties are studied. It is shown that exponential tracking performance can be achieved if the reference input is sufficiently rich and subsystem switches are sufficiently slow. Simulation results with the proposed adaptive SFST scheme applied to the linearized and the nonlinear NASA generic transport model (GTM) are presented.

In Chapter 6, direct adaptive state feedback for output tracking (SFOT) MRAC designs are presented for piecewise linear systems to achieve closed-loop stability and a small output tracking error (in the mean square sense). Asymptotic tracking performance is shown to be achievable under additional plant-model matching conditions. Simulation results on linearized NASA GTM models are presented to demonstrate the substantial performance improvement by the proposed schemes, over conventional 
MRAC schemes.

In Chapter 7, the study of adaptive control of piecewise linear systems is further applied to output feedback designs for output tracking (OFOT). An existing MRAC controller structure in the literature for such systems is reparameterized, and a new adaptive control scheme is proposed. A distinguishing feature of this scheme is the avoidance of controller parameter swapping, which is undesirable because of the additional modeling error introduced in the estimation error signal, used for adaptive law design. Effectiveness of the proposed scheme is validated by simulation results on linearized NASA GTM models.

Chapter 8 concludes the dissertation, and recommendations for future research topics are provided. 


\section{Chapter 2}

\section{Basic Problem Formulation and Background}

\subsection{Basic Problem Formulation}

In this chapter, the basic problems considered in this dissertation are formulated, including the gain margins of adaptive control systems, the performance robustness of model reference adaptive control (MRAC) systems with respect to reduced actuator effectiveness, and adaptive control of piecewise linear systems. Some background on the adaptive control methodology and multivariable adaptive control is provided.

\subsubsection{Gain Margins of Adaptive Control Systems}

The gain margin (GM) concept was originally defined for linear time-invariant (LTI) control systems to specify a necessary and sufficient range (relative to a chosen value) of a control gain for stability $[7,8]$. As a suitable metric for stability and performance, it is also important for adaptive control systems, useful in evaluating and comparing adaptive controllers and maturing adaptive control designs for implementation on performance-critical systems.

Consider an $M$-input, $M$-output linear time-invariant system with a transfer ma- 
trix representation as follows:

$$
\boldsymbol{y}(t)=\boldsymbol{G}(D)[\boldsymbol{u}](t)
$$

where $\boldsymbol{u}(t), \boldsymbol{y}(t) \in \mathbb{R}^{M}$ are the system input and output vectors, $\boldsymbol{G}(D)=\boldsymbol{Z}(D) \boldsymbol{P}^{-1}(D)$ is strictly proper and full rank, and $\boldsymbol{Z}(D), \boldsymbol{P}(D) \in \mathbb{R}^{M \times M}$ are right coprime polynomial matrices with $\boldsymbol{P}(D)$ being column proper. The symbol $D$ is used, in the continuoustime case, as the time-differentiation operator: $D[x](t)=\dot{x}(t), t \in[0,+\infty)$, or in the discrete-time case, as the time-advance operator: $D[x](t)=x(t+1), t \in\{0,1,2, \ldots\}$.

Figure 2.1 depicts the scenario we are considering in the gain margin study of adaptive control systems. An uncertain positive definite and diagonal gain matrix

$$
\boldsymbol{K}=\operatorname{diag}\left\{k_{1}, k_{2}, \ldots, k_{M}\right\}, \quad k_{i}>0, \quad i=1,2, \ldots, M
$$

is present in the forward loop between the controlled plant $\boldsymbol{G}(D)$ and the adaptive controller, denoted as the blocks $\boldsymbol{C}_{1}(D)$ and $\boldsymbol{C}_{2}(D)$. The adaptive controller is designed for $\boldsymbol{K}=\boldsymbol{I}_{M}$ (the $M \times M$ identity matrix) to ensure closed-loop stability (signal boundedness) and asymptotic output tracking performance; that is, $\boldsymbol{y}(t)$ asymptotically tracks a desired trajectory $\boldsymbol{y}_{m}(t)$ generated from a reference model system

$$
\boldsymbol{y}_{m}(t)=\boldsymbol{W}_{m}(D)[\boldsymbol{r}](t)
$$

where $\boldsymbol{W}_{m}(D) \in \mathbb{R}^{M \times M}$ is a rational transfer matrix and $\boldsymbol{r}(t) \in \mathbb{R}^{M}$ is a bounded, piecewise continuous reference input signal.

The gain margin problem for adaptive control systems is formulated as the problem of specifying the stability ranges of $k_{i}$ in (2.1.2), such that under any initial conditions, the designed adaptive control scheme still ensures the closed-loop signal boundedness and output tracking when $\boldsymbol{K}$ is within this range. 


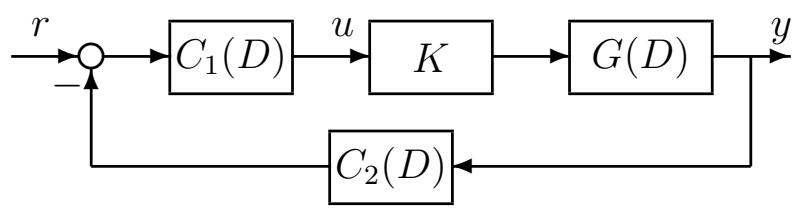

Figure 2.1: Adaptive control system with a gain matrix $K$.

In this dissertation, the gain margins of adaptive control systems with a constant positive definite gain matrix $\boldsymbol{K}$ are studied. The gain margin problem is addressed in Chapter 3.

\subsubsection{Performance Robustness of MRAC with respect to Re- duced Actuator Effectiveness}

In the study of the performance robustness properties of MRAC, the reduction in actuator effectiveness is modeled by an uncertain gain matrix $\boldsymbol{K}$ representing the actuator effectiveness at the control inputs.

Consider Figure 2.1, where $\boldsymbol{G}(D)$ is the controlled plant (2.1.1), for which an adaptive controller $\left(\boldsymbol{C}_{1}(D), \boldsymbol{C}_{2}(D)\right)$ has been designed for the plant output $\boldsymbol{y}(t)$ to asymptotically track a reference trajectory $\boldsymbol{y}_{m}(t)$ generated from (2.1.3), for $\boldsymbol{K}=\boldsymbol{I}_{M}$, i.e., without reduction in actuator effectiveness.

Our objective is to study whether or not the designed adaptive controller still ensures the desired closed-loop performance in the presence of reduction in actuator effectiveness, i.e., in the presence of

$$
\boldsymbol{K}=\operatorname{diag}\left\{k_{1}, \ldots, k_{M}\right\}, 0<k_{i} \leq 1, \quad i=1,2, \ldots, M
$$

It is clear that the gain margin (GM) problem formulation in Section 2.1.1 is similar to that of the performance robustness problem considered here, with the 
difference in that the control gain variations are not bounded from top by 1, i.e., $k_{i}>0, i=1,2, \ldots, M$ in (2.1.2). In Chapter 4 , the gain margin results are applied to address the performance robustness problem.

\subsubsection{Adaptive Control of Piecewise Linear Systems}

To motivate the adaptive control problem for piecewise linear systems, we first present the linearization and approximation of a nonlinear system at multiple operating points by a piecewise linear system.

Consider a nonlinear system

$$
\dot{\boldsymbol{x}}(t)=\boldsymbol{f}(\boldsymbol{x}(t), \boldsymbol{u}(t))
$$

where $\boldsymbol{x}(t) \in \mathbb{R}^{n}$ is the state vector, $\boldsymbol{u}(t) \in \mathbb{R}^{M}$ is the control input vector, and $\boldsymbol{f}(\cdot)$ is an $n$-dimensional nonlinear vector function. Let $\Omega \subset \mathbb{R}^{n+m}$ be the operating region of interest for all possible system state and control vector $(\boldsymbol{x}, \boldsymbol{u})$, and denote $\boldsymbol{x}_{0 i}$ and $\boldsymbol{u}_{0 i}, i \in \mathcal{I} \triangleq\{1,2, \ldots, l\}$, as a set of equilibrium operating points located at some representative (and properly separated) points inside $\boldsymbol{\Omega}$. Introduce a set of $l$ regions $\boldsymbol{\Omega}_{i}$ centered at the chosen operating points $\left(\boldsymbol{x}_{0 i}, \boldsymbol{u}_{0 i}\right)$, and denote their interiors as $\boldsymbol{\Omega}_{i 0}, i \in \mathcal{I}$, such that $\boldsymbol{\Omega}_{j 0} \cap \boldsymbol{\Omega}_{k 0}=\{\emptyset\}$ for all $j \neq k$, and $\cup_{i=1}^{l} \boldsymbol{\Omega}_{i}=\boldsymbol{\Omega}$. With $\boldsymbol{x}_{i}(t)=\boldsymbol{x}(t)-\boldsymbol{x}_{0 i}$ and $\boldsymbol{u}_{i}(t)=\boldsymbol{u}(t)-\boldsymbol{u}_{0 i}$, a set of LTI subsystem models can be obtained, i.e., for $i \in \mathcal{I}$, we have

$$
\dot{\boldsymbol{x}}_{i}(t)=\boldsymbol{A}_{i} \boldsymbol{x}_{i}(t)+\boldsymbol{B}_{i} \boldsymbol{u}_{i}(t), \quad(\boldsymbol{x}(t), \boldsymbol{u}(t)) \in \boldsymbol{\Omega}_{i}
$$

with $\boldsymbol{A}_{i}=\left.\frac{\partial \boldsymbol{f}}{\partial \boldsymbol{x}}\right|_{\left(\boldsymbol{x}_{0 i}, \boldsymbol{u}_{0 i}\right)}, \boldsymbol{B}_{i}=\left.\frac{\partial \boldsymbol{f}}{\partial \boldsymbol{u}}\right|_{\left(\boldsymbol{x}_{0 i}, \boldsymbol{u}_{0 i}\right)}$. Note that at each time instant $t,(\boldsymbol{x}(t), \boldsymbol{u}(t))$ belongs to one and only one $\boldsymbol{\Omega}_{i}$.

To formulate a piecewise linear system model for the nonlinear system (2.1.4), we rewrite (2.1.5) as

$$
\dot{\boldsymbol{x}}(t)=\boldsymbol{A}_{i} \boldsymbol{x}(t)+\boldsymbol{B}_{i} \boldsymbol{u}(t)-\boldsymbol{A}_{i} \boldsymbol{x}_{0 i}-\boldsymbol{B}_{i} \boldsymbol{u}_{0 i}
$$


for $(\boldsymbol{x}(t), \boldsymbol{u}(t)) \in \boldsymbol{\Omega}_{i}$, leading to

$$
\dot{\boldsymbol{x}}(t)=\boldsymbol{A}(t) \boldsymbol{x}(t)+\boldsymbol{B}(t) \boldsymbol{u}(t)-\boldsymbol{A}(t) \boldsymbol{x}_{0}(t)-\boldsymbol{B}(t) \boldsymbol{u}_{0}(t)
$$

where $\boldsymbol{A}(t)=\boldsymbol{A}_{i}, \boldsymbol{B}(t)=\boldsymbol{B}_{i}, \boldsymbol{x}_{0}(t)=\boldsymbol{x}_{0 i}, \boldsymbol{u}_{0}(t)=\boldsymbol{u}_{0 i}$ for $(\boldsymbol{x}(t), \boldsymbol{u}(t)) \in \boldsymbol{\Omega}_{i}$. Note that $\boldsymbol{x}(t)$ is a "global" (instead of perturbed) state vector which is continuous and $\boldsymbol{u}(t)$ is a control input signal to be generated from a control law based on the piecewise linear system model (2.1.6). It can be seen that the parameters in $\boldsymbol{A}(t), \boldsymbol{B}(t)$, and the dynamics offset term $\boldsymbol{f}_{0}(t)=-\boldsymbol{A}(t) \boldsymbol{x}_{0}(t)-\boldsymbol{B}(t) \boldsymbol{u}_{0}(t)$ vary in a piecewise constant pattern; that is, depending on $(\boldsymbol{x}(t), \boldsymbol{u}(t)) \in \boldsymbol{\Omega}_{i}$, they take on different values as specified by the parameter matrix sets $\left(\boldsymbol{A}_{i}, \boldsymbol{B}_{i}\right)$ and operating points $\left(\boldsymbol{x}_{0 i}, \boldsymbol{u}_{0 i}\right)$, where $\boldsymbol{A}_{i}$ and $\boldsymbol{B}_{i}$ are unknown but constant parameter matrices representing the controlled system dynamics at the operating point $\left(\boldsymbol{x}_{0 i}, \boldsymbol{u}_{0 i}\right)$.

To use a linearization-based method for controlling the nonlinear system (2.1.4), it is crucial to design effective control schemes for the piecewise linear system model (2.1.6). Note that every possible state trajectory of the piecewise linear system with state-dependent subsystem switches in (2.1.6) is also a solution of the piecewise linear system in the form of (2.1.6) with time-dependent subsystem switches for a certain switching time sequence [1], such that the latter can be regarded as more generalized than the former in this sense. Furthermore, in the context of model reference adaptive control, the piecewise constant dynamics offset term $\boldsymbol{f}_{0}(t)$ can be taken care of under certain matching conditions with modifications to the adaptive control scheme. Therefore, in this dissertation we will focus on the development of MRAC designs for the following piecewise linear system with time-dependent subsystem switches:

$$
\begin{aligned}
& \dot{\boldsymbol{x}}(t)=\boldsymbol{A}(t) \boldsymbol{x}(t)+\boldsymbol{B}(t) \boldsymbol{u}(t), \quad \boldsymbol{x}\left(t_{0}\right)=\boldsymbol{x}_{0} \\
& \boldsymbol{y}(t)=\boldsymbol{C}^{\boldsymbol{\top}} \boldsymbol{x}(t)
\end{aligned}
$$


where $\boldsymbol{x}(t) \in \mathbb{R}^{n}$ is the state vector, $\boldsymbol{y}(t) \in \mathbb{R}^{M}$ is the system output, $\boldsymbol{u}(t) \in \mathbb{R}^{M}$ is the control input to be specified by an adaptive control law, and $\boldsymbol{A}(t) \in \mathbb{R}^{n \times n}$ and $\boldsymbol{B}(t) \in \mathbb{R}^{n \times M}$ are unknown time-varying parameter matrices that vary in a piecewise constant pattern; that is, during different time periods, $(\boldsymbol{A}(t), \boldsymbol{B}(t))$ take on different values as specified by the parameter matrix sets $\left(\boldsymbol{A}_{i}, \boldsymbol{B}_{i}\right)$, where $\boldsymbol{A}_{i}, \boldsymbol{B}_{i}$ are unknown but constant parameter matrices, and $l$ is the total number of subsystems. The indicator functions are introduced to characterize such a time-varying behavior of the system.

Indicator functions. The indicator functions $\chi_{i}(t)$, which contain the knowledge of the system parameter discontinuities, are assumed to be known and defined as

$$
\chi_{i}(t)= \begin{cases}1, & \text { if }(\boldsymbol{A}(t), \boldsymbol{B}(t))=\left(\boldsymbol{A}_{i}, \boldsymbol{B}_{i}\right) \\ 0, & \text { otherwise }\end{cases}
$$

Since at each specific time instant $t$, only one subsystem is active, the indicator functions defined as above have the following properties:

$$
\sum_{i=1}^{l} \chi_{i}(t)=1, \quad \chi_{j}(t) \chi_{k}(t)=0, \quad j \neq k, \quad j, k \in \mathcal{I}
$$

The indicator functions contain knowledge of the durations of time the system resides in each subsystem and the time instants at which subsystem switches occur, which is useful for adaptive control design. It is assumed that the switches can be detected instantaneously; that is, although $\chi_{i}(t)$ may not be known a priori, they are available during system operation.

With the indicator functions, the system parameter matrices can be expressed as

$$
\boldsymbol{A}(t)=\sum_{i=1}^{l} \boldsymbol{A}_{i} \chi_{i}(t), \quad \boldsymbol{B}(t)=\sum_{i=1}^{l} \boldsymbol{B}_{i} \chi_{i}(t)
$$

The control objective is to design an adaptive state (or output) feedback control law $\boldsymbol{u}(t)$ for closed-loop stability and state (or output) tracking by the piecewise linear 
system state $\boldsymbol{x}(t)$ (output $\boldsymbol{y}(t))$ of a reference trajectory $\boldsymbol{x}_{m}(t)\left(\boldsymbol{y}_{m}(t)\right.$ ), generated from a reference model system.

In Chapters 5-7, adaptive state feedback for state tracking, state feedback for output tracking, and output feedback for output tracking MRAC designs are developed for piecewise linear systems.

\subsection{Background}

A brief introduction to the adaptive control methodology is presented in this section, followed by some background on gain matrix decompositions for multivariable adaptive control designs.

\subsubsection{Adaptive Control Methodology}

Motivated by the autopilot design for high performance aircraft systems in the 1950s, the research in adaptive control has experienced significant theoretical advances and achieved remarkable successes in its application to process control, aircraft control, vehicle control, power systems, and robotic manipulators [9-15]. Adaptive control methodology provides adaptation mechanisms that automatically adjust controller parameters based on closed-loop system performance for a system under parametric, structural, or environmental uncertainties to achieve desired system stability and tracking performance.

An adaptive controller structure is motivated from the nominal control law with parameters of the controlled system assumed to be known. A parameter estimator (called adaptive laws) provides the estimates of the unknown parameters to replace the (unknown) nominal parameters for online control implementation. Depending on what parameter estimates are generated, adaptive control designs can be classified into direct adaptive control and indirect adaptive control. The difference lies in that 
the estimated parameters are either those of the controller (direct) or those of the controlled system (indirect). The way the nominal control law is designed gives rise to two different control design methods: model reference adaptive control (MRAC) and adaptive pole placement control (APPC). The APPC is derived based on the regulation problem and pole placement control, and is applicable to nonminimum-phase systems. As a main and matured approach of adaptive control, the MRAC is used for minimum-phase systems, in which a reference model system is specified that generates desired system output trajectories, and the controller parameters are adaptively updated so that the closed-loop system outputs follow the reference trajectories.

To help understand the basic ideas and the typical design procedure of a model reference adaptive control system, we consider a first-order linear time-invariant system

$$
\dot{y}(t)=a y(t)+u(t), \quad y(0)=y_{0}
$$

where $a, y(t), u(t) \in \mathbb{R}$ are respectively an unknown constant parameter, the controlled output, and the system input. The control objective is to design a feedback control law $u(t)$ such that $y(t)$ tracks a reference trajectory $y_{m}(t) \in \mathbb{R}$ asymptotically, which is generated by the reference model system

$$
\dot{y}_{m}(t)=a_{m} y_{m}(t)+r(t), \quad y_{m}(0)=y_{m 0}
$$

where $a_{m}<0$ is a known constant and $r(t) \in \mathbb{R}$ is a piecewise continuous and bounded reference input, i.e., $r(t) \in \mathcal{L}^{\infty}$. With $a_{m}<0$, the reference model system (2.2.2) is stable and represents the desired dynamic behavior for the controlled system (2.2.1) to follow. Note that (2.2.1) may be unstable.

Nominal control design. If the system parameter a were known, the nominal control law

$$
u(t)=k^{*} y(t)+r(t), \quad k^{*}=a_{m}-a
$$


will result in the closed-loop system

$$
\dot{y}(t)=a_{m} y(t)+r(t)
$$

The equation $k^{*}=a_{m}-a$ is called a matching condition, because under such a condition the closed-loop system (2.2.4) is "matched" to the reference model system (2.2.2). With the definition of the tracking error $e(t)=y(t)-y_{m}(t)$ and its initial value $e(0)=y_{0}-y_{m 0}$, subtracting (2.2.2) from (2.2.4) leads to the error dynamics:

$$
\dot{e}(t)=a_{m} e(t), \quad e(0)=y_{0}-y_{m 0}
$$

whose solution is $e(t)=e^{a_{m} t} e(0)$. We can see that the tracking error decays to zero exponentially.

Adaptive control design. Since the parameter $a$ is unknown, the nominal control law (2.2.3) cannot be implemented because the feedback gain $k^{*}$ is not available. In adaptive control, an estimate $k(t)$ is used to replace $k^{*}$ in (2.2.3) to form the adaptive control law

$$
u(t)=k(t) y(t)+r(t)
$$

which results in the closed-loop system

$$
\dot{y}(t)=a_{m} y(t)+r(t)+\tilde{k}(t) y(t), \quad \tilde{k}=k(t)-k^{*}
$$

where $\tilde{k}(t)$ is the parameter estimation error. Subtracting (2.2.2) from (2.2.6) leads to the closed-loop error dynamics

$$
\dot{e}(t)=a_{m} e(t)+\tilde{k}(t) y(t)
$$

which is linear in the tracking error $e(t)$ and parameter estimation error $\tilde{k}(t)$. The task now is to choose an adaptive law to update $k(t)$ online such that closed-loop 
stability (signal boundedness) and asymptotic tracking are ensured with $a$ unknown. For this purpose, consider a Lyapunov function

$$
V(t)=e^{2}(t)+\frac{1}{\gamma} \tilde{k}^{2}(t), \quad \gamma>0
$$

whose time-derivative along the error dynamics $(2.2 .7)$ is

$$
\begin{aligned}
\dot{V}(t) & =2 e(t) \dot{e}(t)+\frac{2}{\gamma} \tilde{k}(t) \dot{\tilde{k}}(t) \\
& =2 a_{m} e^{2}(t)+2 e(t) \tilde{k}(t) y(t)+\frac{2}{\gamma} \tilde{k}(t) \dot{k}(t) \quad\left(\dot{\tilde{k}}(t)=\dot{k}(t)-\dot{k}^{*}=\dot{k}(t)\right)
\end{aligned}
$$

To make $\dot{V}(t) \leq 0$ for $t \geq 0$, we choose the following adaptive law to update $k(t)$ in $(2.2 .5)$ :

$$
\dot{k}(t)=-\gamma e(t) y(t)
$$

where $\gamma>0$ is the adaptation gain and may be chosen arbitrarily. Such an adaptive law leads to

$$
\dot{V}(t)=2 a_{m} e^{2}(t) \leq 0
$$

since $a_{m}<0$. It follows that $V(t) \in \mathcal{L}^{\infty}$, which implies that $e(t), k(t) \in \mathcal{L}^{\infty}$. Since the reference trajectory $y_{m}(t)$ is bounded due to the stability of the reference model system (2.2.2), we can conclude that $y(t)=y_{m}(t)+e(t) \in \mathcal{L}^{\infty}$. Thus all closedloop signals $y(t), y_{m}(t), k(t)$, and $u(t)$ are bounded, such that the closed-loop system stability is ensured. Furthermore, integrating both sides of (2.2.9) from $t=0$ to $t=\infty$, we have

$$
\int_{0}^{\infty} e^{2}(t) d t=\frac{1}{2 a_{m}}(V(\infty)-V(0))
$$

which is finite because of $V(t) \in \mathcal{L}^{\infty}, \forall t \geq 0$. Thus $e(t) \in \mathcal{L}^{2}$, which together with the fact that $\dot{e}(t) \in \mathcal{L}^{\infty}$ (see $\left.(2.2 .7)\right)$, results in asymptotic tracking, i.e., $\lim _{t \rightarrow \infty} e(t)=0$ [15, Lemma 2.14]. 
Compared with exponential tracking of the nominal case (in which precise knowledge of system parameters is required), the MRAC scheme achieves asymptotic tracking but effectively deals with system parametric uncertainties.

\subsubsection{LDU and LDS Decompositions of a Gain Matrix $K_{p}$}

Suppose that $\boldsymbol{K}_{p} \in \mathbb{R}^{M \times M}$ is a nonsingular matrix, and its leading principle minors are denoted as $\Delta_{i}, i=1,2, \ldots, M$. With $\Delta_{i} \neq 0$ and the sign information of $\Delta_{i}$, $\operatorname{sign}\left[\Delta_{i}\right]$, available, the LDU decomposition [15,73] of $\boldsymbol{K}_{p}$ is $\boldsymbol{K}_{p}=\boldsymbol{L} \boldsymbol{D}^{*} \boldsymbol{U}$ for some $M \times M$ unity lower triangular matrix $\boldsymbol{L}$, unity upper triangular matrix $\boldsymbol{U}$, and diagonal matrix

$$
\boldsymbol{D}^{*}=\operatorname{diag}\left\{d_{1}^{*}, d_{2}^{*}, \ldots, d_{M}^{*}\right\}=\operatorname{diag}\left\{\Delta_{1}, \frac{\Delta_{2}}{\Delta_{1}}, \ldots, \frac{\Delta_{M}}{\Delta_{M-1}}\right\}
$$

Its LDS decomposition, $\boldsymbol{K}_{p}=\boldsymbol{L}_{s} \boldsymbol{D}_{s} \boldsymbol{S}$, follows with $\boldsymbol{L}_{s}=\boldsymbol{L} \boldsymbol{D}_{s}\left(\boldsymbol{U}^{\top}\right)^{-1} \boldsymbol{D}_{s}^{-1}, \boldsymbol{S}=$ $\boldsymbol{U}^{\top} \boldsymbol{D}_{s}^{-1} \boldsymbol{D}^{*} \boldsymbol{U}$, and

$$
\boldsymbol{D}_{s}=\operatorname{diag}\left\{\operatorname{sign}\left[\Delta_{1}\right] \gamma_{1}, \operatorname{sign}\left[\frac{\Delta_{2}}{\Delta_{1}}\right] \gamma_{2}, \ldots, \operatorname{sign}\left[\frac{\Delta_{M}}{\Delta_{M-1}}\right] \gamma_{M}\right\}
$$

with $\gamma_{i}>0, i=1,2, \ldots, M$, which can be arbitrary.

In this chapter, we have formulated the basic control problems to be addressed in this dissertation. Some preliminaries are reviewed for a better understanding of the content in subsequent chapters. 


\section{Chapter 3}

\section{Gain Margins of Adaptive Control Systems}

This chapter presents a systematic study of the gain margins for many commonly used adaptive control schemes, based on their stability properties [9-15]. Before presenting the analytical results, it is important to note the difference between the GM of adaptive control systems and that of classical control systems. Adaptive control systems are nonlinear in essence, and the required control performance is asymptotic tracking in addition to stability (signal boundedness). For adaptive control systems, the gain margin defines a range of a control gain variation with which both asymptotic tracking and stability are ensured under any initial conditions. For some values of the gain variation outside the range, the system may still have the desired properties for some initial conditions. While in a non-adaptive control system, a gain variation in the gain margin range can lead to deterioration of system tracking performance, and a gain variation outside the range can make the control system unstable for almost all initial conditions.

This chapter is organized as follows. In Section 3.1, the gain margin problem for adaptive control systems is stated, and a summary of analytical GMs for various adaptive control systems is given. In Sections 3.3 and 3.4, the GM problem is studied 
for direct MRAC schemes, for the continuous-time case and the discrete-time case, respectively, applied to single input, single output (SISO) and multiple input, multiple output (MIMO) systems. As an extension, the GM results of adaptive state feedback control schemes and some adaptive control schemes applied to nonlinear plants are given. The GM of indirect MRAC systems is presented in Section 3.5, and sampleddata adaptive control systems are considered in Section 3.6. An illustrative simulation study is provided in Section 3.7.

\subsection{Problem Statement}

The gain margin problem is stated in this section, followed by a summary of the analytical GM results for different adaptive control designs. The standard multivariable MRAC assumptions, including those based on the high frequency gain matrix decompositions, are also presented.

Consider Figure 3.1, in which $\boldsymbol{G}(D)$ is the transfer matrix of the $M$-input, $M$ output linear time-invariant controlled system:

$$
\boldsymbol{y}(t)=\boldsymbol{G}(D)[\boldsymbol{u}](t)
$$

where $\boldsymbol{u}(t), \boldsymbol{y}(t) \in \mathbb{R}^{M}$ are the system input and output vectors, $\boldsymbol{G}(D)=\boldsymbol{Z}(D) \boldsymbol{P}^{-1}(D)$ is strictly proper and full rank, and $\boldsymbol{Z}(D), \boldsymbol{P}(D) \in \mathbb{R}^{M \times M}$ are right coprime polynomial matrices with $\boldsymbol{P}(D)$ being column proper. The symbol $D$ is used, in the continuoustime case, as the time-differentiation operator: $D[x](t)=\dot{x}(t), t \in[0,+\infty)$, or in the discrete-time case, as the time-advance operator: $D[x](t)=x(t+1), t \in\{0,1,2, \ldots\}$.

An uncertain constant, positive definite and diagonal gain matrix

$$
\boldsymbol{K}=\operatorname{diag}\left\{k_{1}, k_{2}, \ldots, k_{M}\right\}, \quad k_{i}>0, \quad i=1,2, \ldots, M
$$

is present in the forward loop between the controlled plant $\boldsymbol{G}(D)$ and the adaptive 
controller, denoted as the blocks $\boldsymbol{C}_{1}(D)$ and $\boldsymbol{C}_{2}(D)$. The adaptive controller is designed for $\boldsymbol{K}=\boldsymbol{I}_{M}$ (the $M \times M$ identity matrix) to ensure closed-loop stability (signal boundedness) and asymptotic output tracking performance; that is, $\boldsymbol{y}(t)$ asymptotically tracks a desired trajectory $\boldsymbol{y}_{m}(t)$ generated from a reference model system

$$
\boldsymbol{y}_{m}(t)=\boldsymbol{W}_{m}(D)[\boldsymbol{r}](t)
$$

where $\boldsymbol{W}_{m}(D) \in \mathbb{R}^{M \times M}$ is a rational transfer matrix and $\boldsymbol{r}(t) \in \mathbb{R}^{M}$ is a bounded, piecewise continuous reference input signal.

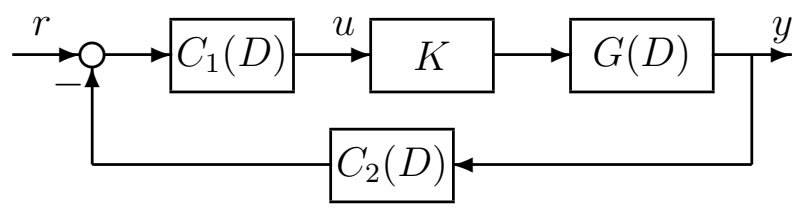

Figure 3.1: Adaptive control system with a gain matrix $K$.

The gain margin problem for adaptive control systems is formulated as the problem of specifying the stability ranges of $k_{i}$ in (3.1.2), such that under any initial conditions, the designed adaptive control scheme still ensures the closed-loop signal boundedness and output tracking when $\boldsymbol{K}$ is within this range.

The analytical gain margin results are summarized in Table 3.1 for both continuoustime (CT) and discrete-time (DT) cases. The results of multivariable direct MRAC are derived for the designs based on the LDS decomposition of the system high frequency gain matrix $\boldsymbol{K}_{p}$, and those of multivariable state feedback for state tracking (SFST) scheme are for the design based on the LDU parametrization. For GM results of single input, single output (SISO) cases with $\boldsymbol{K}=k, k_{p}^{0}$ and $k_{p 0}$ are the upper and lower bounds of the magnitude of system high frequency gain $k_{p}$, respectively, used in the design of the adaptive control schemes [15,62]. 
Table 3.1: Gain margins of adaptive control systems

\begin{tabular}{ll}
\hline \hline Adaptive control scheme & Gain margin \\
\hline CT MIMO direct MRAC & $k_{i} \in(0,+\infty), i=1,2, \ldots, M$ \\
CT SISO direct MRAC & $k \in(0,+\infty)$ \\
DT MIMO direct MRAC & $k_{i} \in\left(0, k_{i}^{0}\right], \mathrm{a}, i=1,2, \ldots, M$ \\
DT SISO direct MRAC & $k \in\left(0, \frac{k_{p}^{0}}{\left|k_{p}\right|}\right], 0<\left|k_{p}\right| \leq k_{p}^{0}$ \\
MIMO indirect MRAC & $k_{i} \in\left[k_{i 0},+\infty\right), i=1,2, \ldots, M$ \\
SISO indirect MRAC & $k \in\left[\frac{k_{p 0}}{\left|k_{p}\right|},+\infty\right), 0<k_{p 0} \leq\left|k_{p}\right|$ \\
CT MIMO SFST & $k_{i} \in(0,+\infty), i=1,2, \ldots, M$ \\
CT SISO SFST & $k \in(0,+\infty)$ \\
CT SISO SFOT & $k \in(0,+\infty)$ \\
DT SISO SFOT & $k \in\left(0, \frac{k_{p}^{0}}{\left|k_{p}\right|}\right], 0<\left|k_{p}\right| \leq k_{p}^{0}$ \\
\hline
\end{tabular}

a The stability ranges of the gains $k_{i}, i=1,2, \ldots, M$, have some finite upper bounds which depend on some upper bounds of the system high frequency gain matrix (used in adaptive laws for parameter adaptation), as well as on some coupling terms of $K_{p}$.

b The stability ranges of the gains $k_{i}, i=1,2, \ldots, M$, have some positive lower bounds which depend on some lower bounds of the system high frequency gain matrix $K_{p}$ (used in adaptive laws for plant identification).

The high frequency gain matrix of the controlled system is defined as

$$
\boldsymbol{K}_{p}=\lim _{D \rightarrow \infty} \boldsymbol{\xi}_{m}(D) \boldsymbol{G}(D)
$$

with $\boldsymbol{\xi}_{m}(D)$ being the modified interactor matrix of $\boldsymbol{G}(D)$. For MRAC designs based on decompositions of $\boldsymbol{K}_{p}$, the reference model transfer matrix $\boldsymbol{W}_{m}(D)$ in (3.1.3) is chosen to be $\boldsymbol{W}_{m}(D)=\boldsymbol{\xi}_{m}^{-1}(D)$.

MRAC Assumptions. For MRAC designs, the standard assumptions are:

(A3.1-1) All zeros of $\boldsymbol{G}(D)$ are stable;

(A3.1-2) The observability index $\nu$ of $\boldsymbol{G}(D)$ is known; 
(A3.1-3) A modified interactor matrix $\boldsymbol{\xi}_{m}(D)$, which has a stable inverse, of $\boldsymbol{G}(D)$ is known; and

(A3.1-4) All leading principal minors of $\boldsymbol{K}_{p}, \Delta_{i}, i=1,2, \ldots, M$, are nonzero with known signs.

Besides, for discrete-time designs, we assume:

(A3.1-4D) The upper bounds $d_{i}^{0}$ of $\left|d_{i}^{*}\right|=\left|\Delta_{i} / \Delta_{i-1}\right|$ with $\Delta_{0}=1$, such that $0<$ $\left|d_{i}^{*}\right| \leq d_{i}^{0}, i=1,2, \ldots, M$, are known.

\subsection{LDU Decomposition of $K_{p} K$}

With Assumption (A3.1-4), the LDU decomposition of $\boldsymbol{K}_{p}, \boldsymbol{K}_{p}=\boldsymbol{L} \boldsymbol{D}^{*} \boldsymbol{U}$ and its LDS decomposition, $\boldsymbol{K}_{p}=\boldsymbol{L}_{s} \boldsymbol{D}_{s} \boldsymbol{S}$, have been presented as in Section 2.2.2. In the presence of $\boldsymbol{K} \neq \boldsymbol{I}_{M}$, the controlled system becomes

$$
\boldsymbol{y}(t)=\boldsymbol{G}(D) \boldsymbol{K}[\boldsymbol{u}](t)
$$

and its high frequency gain matrix is $\boldsymbol{K}_{p} \boldsymbol{K}$. The following lemma establishes the connection between the LDU decomposition of $\boldsymbol{K}_{p}$ and that of $\boldsymbol{K}_{p} \boldsymbol{K}$, which is key to the gain margin analysis for both continuous-time and discrete-time multivariable MRAC systems designed based on the LDS decomposition of $\boldsymbol{K}_{p}$.

Lemma 3.2.1. The gain matrix $\boldsymbol{K}_{p} \boldsymbol{K} \in \mathbb{R}^{M \times M}$, where $\boldsymbol{K}_{p}$ is nonsingular with nonzero leading principle minors and $\boldsymbol{K}=\operatorname{diag}\left\{k_{1}, k_{2}, \ldots, k_{M}\right\}>\mathbf{0}$, has a unique $L D U$ decomposition

$$
\boldsymbol{K}_{p} \boldsymbol{K}=\overline{\boldsymbol{L}} \overline{\boldsymbol{D}}^{*} \overline{\boldsymbol{U}}, \quad \overline{\boldsymbol{L}}=\boldsymbol{L}, \quad \overline{\boldsymbol{D}}^{*}=\boldsymbol{D}^{*} \boldsymbol{K}, \overline{\boldsymbol{U}}=\boldsymbol{K}^{-1} \boldsymbol{U} \boldsymbol{K}
$$

where $\boldsymbol{K}_{p}=\boldsymbol{L} \boldsymbol{D}^{*} \boldsymbol{U}$ is the $L D U$ decomposition of $\boldsymbol{K}_{p}$. 
Proof: Suppose the matrix $\boldsymbol{K}_{p}$ is represented as $K_{p}=\left[k_{p i j}\right], i, j=1,2, \ldots, M$, and its nonzero leading principle minors are $\Delta_{i}, i=1,2, \ldots, M$. With the diagonal and positive definite gain matrix $\boldsymbol{K}$ in (3.1.2), we have the leading principle minors of $\boldsymbol{K}_{p} \boldsymbol{K}$ as:

$$
\bar{\Delta}_{i}=\Delta_{i} \prod_{j=1}^{i} k_{j}, i=1,2, \ldots, M
$$

from which and $\boldsymbol{D}^{*}$ in $(2.2 .10)$, we can obtain

$$
\overline{\boldsymbol{D}}^{*}=\operatorname{diag}\left\{\bar{\Delta}_{1}, \frac{\bar{\Delta}_{2}}{\bar{\Delta}_{1}}, \ldots, \frac{\bar{\Delta}_{M}}{\bar{\Delta}_{M-1}}\right\}=\boldsymbol{D}^{*} \boldsymbol{K}
$$

Therefore, based on the fact that

$$
\boldsymbol{K}_{p} \boldsymbol{K}=\boldsymbol{L} \boldsymbol{D}^{*} \boldsymbol{U} \boldsymbol{K}=\overline{\boldsymbol{L}} \boldsymbol{D}^{*} \boldsymbol{K} \overline{\boldsymbol{U}}
$$

we conclude that $\overline{\boldsymbol{L}}=\boldsymbol{L}$ and $\overline{\boldsymbol{U}}=\boldsymbol{K}^{-1} \boldsymbol{U} \boldsymbol{K}$, where $\overline{\boldsymbol{L}}$ and $\overline{\boldsymbol{U}}$ are unity lower triangular and unity upper triangular matrices, respectively. Furthermore, this decomposition is unique because of the uniqueness of matrix LDU decomposition.

From Lemma 3.2.1, we conclude that the leading principle minors of $\boldsymbol{K}_{p} \boldsymbol{K}$ are also nonzero, and their sign information is the same as that of the leading principle minors of $\boldsymbol{K}_{p}$. This result will be useful in clarifying the GM of both continuous-time and discrete-time multivariable MRAC systems.

In the reminder of this chapter, the gain margins of various adaptive control systems are analyzed. We first show by proof that the GM for continuous-time direct MRAC systems is infinity. While this GM result is intuitively understandable in the sense that MRAC can handle large parameter uncertainties, the GM analysis of discrete-time direct MRAC systems is technically more involved and provides more insight into the gain margin concept for adaptive control systems; in particular, the GM for such systems is finite with an upper bound. These GM results are applicable 
to both output feedback and state feedback designs for output tracking (for state tracking as well in the continuous-time case). They are also applicable to nonlinear adaptive control systems using feedback linearization designs [74] or backstepping designs [13]. In addition, by considering the GM for sampled-data systems, we investigate the effect of sample time on GM, and we will show that as the sample time approaches zero, the GM tends to infinity, which relates the GM results for both continuous-time and discrete-time MRAC systems. For indirect MRAC of multivariable systems, the gain margin problem is formulated in terms of the nonsingular estimation problem of a system high frequency gain matrix using system input and output measurements. The GM for such adaptive control systems turns out to have a lower bound. This situation is also true for an indirect adaptive pole placement design. As it will be shown later, the upper or lower bound for the GM depends on some design parameters in the adaptive control scheme, and thus can be enlarged by a proper choice of these design parameters.

\subsection{Gain Margins of Continuous-Time MRAC Sys- tems}

For a continuous-time direct MRAC system, its gain margin can be derived based on the adaptive control scheme applied. We will present the GM results for MRAC designs for both SISO and MIMO controlled systems. The result of the multivariable case is for the design based on the LDS decomposition of $\boldsymbol{K}_{p}$. As a comparison, the non-adaptive multivariable model reference control problem and the corresponding GM analysis are presented first. 


\subsubsection{Model Reference Control System}

When the parameters of the plant in (3.1.1) are known, the above stated MRAC problem reduces to model reference control (MRC), i.e., the non-adaptive control problem.

The model reference controller

$$
\boldsymbol{u}(t)=\boldsymbol{\Theta}_{1}^{* \boldsymbol{T}} \boldsymbol{\omega}_{1}(t)+\boldsymbol{\Theta}_{2}^{* \top} \boldsymbol{\omega}_{2}(t)+\boldsymbol{\Theta}_{20}^{*} \boldsymbol{y}(t)+\boldsymbol{\Theta}_{3}^{*} \boldsymbol{r}(t)
$$

with controller parameters computed from the plant-model transfer matrix matching equation

$$
\boldsymbol{\Theta}_{1}^{* \top} \boldsymbol{A}(D) \boldsymbol{P}(D)+\left(\boldsymbol{\Theta}_{2}^{* \top} \boldsymbol{A}(D)+\Lambda(D) \boldsymbol{\Theta}_{20}^{*}\right) \boldsymbol{Z}(D)=\Lambda(D)\left(\boldsymbol{P}(D)-\boldsymbol{\Theta}_{3}^{*} \boldsymbol{\xi}_{m}(D) \boldsymbol{Z}(D)\right)
$$

achieves closed-loop signal boundedness and asymptotic output tracking, where

$$
\begin{aligned}
\boldsymbol{\omega}_{1}(t) & =\boldsymbol{F}(D)[\boldsymbol{u}](t), \quad \boldsymbol{\omega}_{2}(t)=\boldsymbol{F}(D)[\boldsymbol{y}](t) \\
\boldsymbol{F}(D) & =\frac{\boldsymbol{A}(D)}{\Lambda(D)}, \quad \boldsymbol{A}(D)=\left[\boldsymbol{I}_{M}, D \boldsymbol{I}_{M}, \ldots, D^{\nu-2} \boldsymbol{I}_{M}\right]^{\top}
\end{aligned}
$$

for a stable monic polynomial $\Lambda(D)$ of degree $\nu-1$, and $\Theta_{i}^{*}, i=1,2,20,3$, are of appropriate dimensions.

$$
\begin{aligned}
& \text { With } \boldsymbol{\Theta}_{3}^{*}=\boldsymbol{K}_{p}^{-1} \text { and } \boldsymbol{K}_{p}=\boldsymbol{L}_{s} \boldsymbol{D}_{s} \boldsymbol{S},(3.3 .2) \text { leads to } \\
& \boldsymbol{D}_{s} \boldsymbol{S}\left(\boldsymbol{u}(t)-\boldsymbol{\Theta}_{1}^{* \boldsymbol{\top}} \boldsymbol{\omega}_{1}(t)-\boldsymbol{\Theta}_{2}^{* \boldsymbol{\top}} \boldsymbol{\omega}_{2}(t)-\boldsymbol{\Theta}_{20}^{*} \boldsymbol{y}(t)-\boldsymbol{\Theta}_{3}^{*} \boldsymbol{r}(t)\right)=\boldsymbol{L}_{s}^{-1} \boldsymbol{\xi}_{m}(D)\left[\boldsymbol{y}-\boldsymbol{y}_{m}\right](t)
\end{aligned}
$$

Hence, by applying the model reference control (3.3.1) to the plant (3.1.1), we obtain $\boldsymbol{\xi}_{m}(D)\left[\boldsymbol{y}-\boldsymbol{y}_{m}\right](t)=0$; that is, $\lim _{t \rightarrow \infty}\left(\boldsymbol{y}(t)-\boldsymbol{y}_{m}(t)\right)=0$ exponentially.

We briefly study the gain margin problem for this MRC design. When the gain matrix $\boldsymbol{K} \neq \boldsymbol{I}_{M}$, the new controlled plant in Fig. 3.1 becomes

$$
\boldsymbol{y}(t)=\boldsymbol{G}(D) \boldsymbol{K}[u](t), \quad \boldsymbol{G}(D)=\boldsymbol{Z}(D) \boldsymbol{P}^{-1}(D)
$$


After multiplying both sides of (3.3.2) by $\frac{1}{\Lambda(D)} \boldsymbol{P}^{-1}(D) \boldsymbol{K}$ and applying it on $\boldsymbol{u}(t)$, we obtain

$$
\begin{aligned}
& \left(\left(\boldsymbol{K} \boldsymbol{\Theta}_{1}^{* \top} \boldsymbol{F}(D) \boldsymbol{K}^{-1}-\boldsymbol{\Theta}_{1}^{* \top} \boldsymbol{F}(D)\right) \boldsymbol{P}(D) \boldsymbol{Z}^{-1}(D)\right. \\
& \left.+\left(\boldsymbol{K}-\boldsymbol{I}_{M}\right)\left(\boldsymbol{\Theta}_{2}^{* \top} \boldsymbol{F}(D)+\boldsymbol{\Theta}_{20}^{*}\right)-\boldsymbol{\Theta}_{3}^{*} \boldsymbol{W}_{m}^{-1}(D)\right)[\boldsymbol{y}](t) \\
= & -\boldsymbol{K} \boldsymbol{\Theta}_{3}^{*} \boldsymbol{W}_{m}^{-1}(D)\left[\boldsymbol{y}_{m}\right](t)
\end{aligned}
$$

As can be seen from (3.3.5), the mismatch between the plant (3.3.4) and the reference model (3.1.3) for all $\boldsymbol{K} \neq \boldsymbol{I}_{M}$ results in the loss of the asymptotic output tracking property. Therefore, the gain margin is 1 for closed-loop tracking; that is, for any gain matrix $\boldsymbol{K} \neq \boldsymbol{I}_{M}$, asymptotic output tracking cannot be achieved with a model reference controller designed for $\boldsymbol{K}=\boldsymbol{I}_{M}$. However, if the gain matrix $\boldsymbol{K}$ is such that the left-hand-side operator on $\boldsymbol{y}(t)$ in (3.3.5) is stable, we have $\boldsymbol{y}(t) \in \mathcal{L}^{\infty}$, and by (3.3.4) and (3.3.5), $\boldsymbol{u}(t) \in \mathcal{L}^{\infty}$, thus $\boldsymbol{\omega}_{1}(t) \in \mathcal{L}^{\infty}$ and $\boldsymbol{\omega}_{2}(t) \in \mathcal{L}^{\infty}$. In other words, all signals in the closed-loop system are bounded, and the system is stable.

Remark 3.3.1. For non-adaptive control systems, closed-loop stability is robust with respect to some gain variations. However, tracking requires exact knowledge of the plant parameters, any variations of which can corrupt the desired tracking performance. In other words, in terms of tracking, non-adaptive control systems are not robust with regard to gain parameter changes. In contrast, MRAC systems can deal with this kind of changes, not surprisingly. More specifically, a continuous-time MIMO direct MRAC system has an infinite gain margin, while its discrete-time counterpart has a finite gain margin, to be specific, it has an upper bound for gain margin. Furthermore, indirect MRAC systems have lower bounds for their gain margins. 


\subsubsection{Design for SISO Systems}

The continuous-time plant model is (3.1.1) with $D$ replaced by $s$, i.e.,

$$
y(t)=G(s)[u](t), \quad G(s)=k_{p} \frac{Z(s)}{P(s)}
$$

where $k_{p}$ is the plant high frequency gain, $y(t) \in \mathbb{R}$ is the plant output, $u(t) \in \mathbb{R}$ is the plant input, and

$$
Z(s)=s^{m}+z_{m-1} s^{m-1}+\cdots+z_{1} s+z_{0}, \quad P(s)=s^{n}+p_{n-1} s^{n-1}+\cdots+p_{1} s+p_{0}
$$

are polynomials in $s$ with $z_{i}, i=0,1, \ldots, m-1$, and $p_{j}, j=0,1, \ldots, n-1, n>m$, being unknown but constant parameters.

The reference model system is (3.1.3), where the stable transfer function $W_{m}(s)=$ $P_{m}^{-1}(s)$ with $P_{m}(s)$ being a monic stable polynomial, $y_{m}(t) \in \mathbb{R}$ is the desired reference output, and $r(t) \in \mathbb{R}$ is an external reference input signal.

MRAC Assumptions. For continuous-time SISO MRAC designs, Assumptions (A3.1-1)-(A3.1-4) reduce to:

(A3.3-1) $Z(s)$ is a stable polynomial;

(A3.3-2) An upper bound $\bar{n} \geq n$ for the degree $n$ of $P(s)$ is known;

(A3.3-3) The degree of $P_{m}(s)$ is $n^{*}=n-m>0$; and

(A3.3-4) The sign of $k_{p}$ is known.

Controller Structure. The following adaptive control law is applied:

$$
u(t)=\boldsymbol{\theta}_{1}^{\boldsymbol{\top}}(t) \boldsymbol{\omega}_{1}(t)+\boldsymbol{\theta}_{2}^{\boldsymbol{\top}}(t) \boldsymbol{\omega}_{2}(t)+\theta_{20}(t) y(t)+\theta_{3}(t) r(t)
$$

with $\boldsymbol{\theta}_{1}(t), \boldsymbol{\theta}_{2}(t) \in \mathbb{R}^{\bar{n}-1}, \theta_{20}(t), \theta_{3}(t) \in \mathbb{R}$ to be updated from an adaptive law, and $\boldsymbol{\omega}_{1}(t), \boldsymbol{\omega}_{2}(t)$ defined as

$$
\boldsymbol{\omega}_{1}(t)=\frac{\boldsymbol{a}(s)}{\Lambda(s)}[u](t), \quad \boldsymbol{\omega}_{2}(t)=\frac{\boldsymbol{a}(s)}{\Lambda(s)}[y](t)
$$


where $\boldsymbol{a}(s)=\left[1, s, \ldots, s^{\bar{n}-2}\right]^{\top}$ and $\Lambda(s)$ is a monic stable polynomial of degree $\bar{n}-1$.

If the plant parameters were known, the control objective can be achieved by implementing (3.3.7) with $\boldsymbol{\theta}_{i}(t)=\boldsymbol{\theta}_{i}^{*}, i=1,2,20,3$, where $\boldsymbol{\theta}_{i}^{*}$ are computed from the plant-model matching equation [15]:

$$
\boldsymbol{\theta}_{1}^{* \mathrm{~T}} \boldsymbol{a}(s) P(s)+\left(\boldsymbol{\theta}_{2}^{* \mathrm{~T}} \boldsymbol{a}(s)+\theta_{20}^{*} \Lambda(s)\right) k_{p} Z(s)=\Lambda(s)\left(P(s)-k_{p} \theta_{3}^{*} Z(s) P_{m}(s)\right)
$$

On the other hand, with plant parameters unknown, adaptive laws are designed to update $\boldsymbol{\theta}_{i}(t)$, the time-varying estimates of $\boldsymbol{\theta}_{i}^{*}$, and the adaptive control law (3.3.7) is applied to the plant (3.3.6).

By applying both sides of (3.3.8) on $y(t)$, and substituting (3.3.6) into the resulting equation, after ignoring the initial condition related exponentially decaying term, we can express (3.3.7) as

$$
u(t)=\boldsymbol{\theta}_{1}^{* \top} \boldsymbol{\omega}_{1}(t)+\boldsymbol{\theta}_{2}^{* \top} \boldsymbol{\omega}_{2}(t)+\theta_{20}^{*} y(t)+\theta_{3}^{*} P_{m}(s)[y](t)
$$

In view of the adaptive control law (3.3.7), the tracking error equation follows:

$$
\begin{aligned}
e(t) & =y(t)-y_{m}(t)=\frac{k_{p}}{P_{m}(s)}\left[\tilde{\boldsymbol{\theta}}^{\boldsymbol{\top}} \boldsymbol{\omega}\right](t) \\
& =-k_{p}\left(\boldsymbol{\theta}^{* \boldsymbol{\top}} \frac{1}{P_{m}(s)}[\boldsymbol{\omega}](t)-\frac{1}{P_{m}(s)}\left[\boldsymbol{\theta}^{\boldsymbol{\top}} \boldsymbol{\omega}\right](t)\right)
\end{aligned}
$$

where $\boldsymbol{\theta}(t)=\left[\boldsymbol{\theta}_{1}^{\boldsymbol{\top}}(t), \boldsymbol{\theta}_{2}^{\boldsymbol{\top}}(t), \theta_{20}(t), \theta_{3}(t)\right]^{\boldsymbol{\top}}$ is the time-varying estimate of the nominal controller parameter vector $\boldsymbol{\theta}^{*}=\left[\boldsymbol{\theta}_{1}^{* \top}, \boldsymbol{\theta}_{2}^{* \top}, \theta_{20}^{*}, \theta_{3}^{*}\right]^{\top}, \tilde{\boldsymbol{\theta}}(t)=\boldsymbol{\theta}(t)-\boldsymbol{\theta}^{*}$, and $\boldsymbol{\omega}(t)=$ $\left[\boldsymbol{\omega}_{1}^{\top}(t), \boldsymbol{\omega}_{2}^{\top}(t), y(t), r(t)\right]^{\top}$.

Error Model. Motivated by the tracking error equation (3.3.9), the estimation error signal is defined as

$$
\epsilon(t)=e(t)+\rho(t) \xi(t), \quad \xi(t)=\boldsymbol{\theta}^{\boldsymbol{\top}}(t) \boldsymbol{\zeta}(t)-\frac{1}{P_{m}(s)}\left[\boldsymbol{\theta}^{\boldsymbol{\top}} \boldsymbol{\omega}\right](t), \quad \boldsymbol{\zeta}(t)=\frac{1}{P_{m}(s)}[\boldsymbol{\omega}](t)
$$


where $\rho(t)$ is the estimate of $\rho^{*}=k_{p}$. By substituting (3.3.9) into the above equation, we can obtain the estimation error model as follows:

$$
\epsilon(t)=\tilde{\rho}(t) \xi(t)+\rho^{*} \tilde{\boldsymbol{\theta}}^{\top}(t) \boldsymbol{\zeta}(t), \quad \tilde{\rho}(t)=\rho(t)-\rho^{*}, \quad \tilde{\boldsymbol{\theta}}(t)=\boldsymbol{\theta}(t)-\boldsymbol{\theta}^{*}
$$

Adaptive Law. In view of the estimation error model and by introducing a normalizing signal $m(t)=\sqrt{1+\boldsymbol{\zeta}^{\top}(t) \boldsymbol{\zeta}(t)+\xi^{2}(t)}$, the gradient adaptive laws are applied to update $\boldsymbol{\theta}(t)$ and $\rho(t)$ :

$$
\begin{aligned}
\dot{\boldsymbol{\theta}}(t) & =-\frac{\operatorname{sign}\left[k_{p}\right] \boldsymbol{\Gamma} \epsilon(t) \boldsymbol{\zeta}(t)}{m^{2}(t)}, \quad \boldsymbol{\Gamma}=\boldsymbol{\Gamma}^{\boldsymbol{\top}}>0 \\
\dot{\rho}(t) & =-\frac{\gamma \epsilon(t) \xi(t)}{m^{2}(t)}, \quad \gamma>0
\end{aligned}
$$

For $k=1$, the adaptive control law (3.3.7) with parameters updated by the adaptive laws (3.3.10) ensures that all the signals in the closed-loop system are bounded, and the tracking error reduces to zero asymptotically with time: $\lim _{t \rightarrow \infty}(y(t)-$ $\left.y_{m}(t)\right)=0[15]$.

Gain Margin Analysis. The same closed-loop stability properties and asymptotic tracking performance also hold for any control gain variation $k>0$; that is, we have the following gain margin result:

Proposition 3.3.2. A continuous-time SISO direct MRAC system has gain margin $(0,+\infty)$

Proof: To see this result, we can absorb the gain $k$ into the high frequency gain of the plant, which in this case is a scalar $k_{p}$, and the high frequency gain becomes $k_{p} k$. The parameter $\rho(t)$ becomes the estimate of $\rho^{*}=k_{p} k$ when $k$ deviates from 1 . The only assumption on $k_{p}$ is the known $\operatorname{sign}\left[k_{p}\right]$, and $k>0$ does not violate this assumption. Due to the fact that the adaptive laws (3.3.10) allow any initial estimates of $\boldsymbol{\theta}^{*}$ and $\rho^{*}$, the desired closed-loop performance (signal boundedness and output tracking) is 
still ensured for any constant gain $k>0$; that is, the adaptive control system's GM is $(0,+\infty)$.

\subsubsection{LDS Decomposition Based Design for MIMO Systems}

If the parameters of system $\boldsymbol{G}(s)$ in (3.1.1), i.e.,

$$
\boldsymbol{y}(t)=\boldsymbol{G}(s)[u](t), \quad \boldsymbol{G}(s)=\boldsymbol{Z}(s) \boldsymbol{P}^{-1}(s)
$$

are known, the model reference controller

$$
\boldsymbol{u}(t)=\boldsymbol{\Theta}_{1}^{* \top} \boldsymbol{\omega}_{1}(t)+\boldsymbol{\Theta}_{2}^{* \top} \boldsymbol{\omega}_{2}(t)+\boldsymbol{\Theta}_{20}^{*} \boldsymbol{y}(t)+\boldsymbol{\Theta}_{3}^{*} \boldsymbol{r}(t)
$$

with $\Theta_{i}^{*}, i=1,2,20,3$, computed from the plant-model matching equation

$$
\boldsymbol{\Theta}_{1}^{* \top} \boldsymbol{A}(s) \boldsymbol{P}(s)+\left(\boldsymbol{\Theta}_{2}^{* \top} \boldsymbol{A}(s)+\Lambda(s) \boldsymbol{\Theta}_{20}^{*}\right) \boldsymbol{Z}(s)=\Lambda(s)\left(\boldsymbol{P}(s)-\boldsymbol{\Theta}_{3}^{*} \boldsymbol{\xi}_{m}(s) \boldsymbol{Z}(s)\right)
$$

achieves closed-loop signal boundedness and asymptotic output tracking, where

$$
\begin{aligned}
\boldsymbol{\omega}_{1}(t) & =\boldsymbol{F}(s)[\boldsymbol{u}](t), \quad \boldsymbol{\omega}_{2}(t)=\boldsymbol{F}(s)[\boldsymbol{y}](t) \\
\boldsymbol{F}(s) & =\boldsymbol{A}(s) / \Lambda(s), \quad \boldsymbol{A}(s)=\left[\boldsymbol{I}_{M}, s \boldsymbol{I}_{M}, \ldots, s^{\nu-2} \boldsymbol{I}_{M}\right]^{\top}
\end{aligned}
$$

for a stable monic polynomial $\Lambda(s)$ of degree $\nu-1$, and $\boldsymbol{\Theta}_{i}^{*}, i=1,2,20,3$, are of appropriate dimensions.

$$
\begin{aligned}
& \text { With } \boldsymbol{\Theta}_{3}^{*}=\boldsymbol{K}_{p}^{-1} \text { and } \boldsymbol{K}_{p}=\boldsymbol{L}_{s} \boldsymbol{D}_{s} \boldsymbol{S},(3.3 .12) \text { leads to } \\
& \boldsymbol{D}_{s} \boldsymbol{S}\left(\boldsymbol{u}(t)-\boldsymbol{\Theta}_{1}^{* \top} \boldsymbol{\omega}_{1}(t)-\boldsymbol{\Theta}_{2}^{* \top} \boldsymbol{\omega}_{2}(t)-\boldsymbol{\Theta}_{20}^{*} \boldsymbol{y}(t)-\boldsymbol{\Theta}_{3}^{*} \boldsymbol{r}(t)\right)=\boldsymbol{L}_{s}^{-1} \boldsymbol{\xi}_{m}(s)\left[\boldsymbol{y}-\boldsymbol{y}_{m}\right](t)
\end{aligned}
$$

Hence, by applying the model reference control (3.3.11) to the system (3.1.1), we obtain $\boldsymbol{\xi}_{m}(s)\left[\boldsymbol{y}-\boldsymbol{y}_{m}\right](t)=\mathbf{0}$; that is, $\lim _{t \rightarrow \infty}\left(\boldsymbol{y}(t)-\boldsymbol{y}_{m}(t)\right)=\mathbf{0}$ exponentially.

Controller Structure. When the plant parameters are unknown, the following MRAC control law is applied:

$$
\boldsymbol{u}(t)=\boldsymbol{\Theta}_{1}^{\top}(t) \boldsymbol{\omega}_{1}(t)+\boldsymbol{\Theta}_{2}^{\top}(t) \boldsymbol{\omega}_{2}(t)+\boldsymbol{\Theta}_{20}(t) \boldsymbol{y}(t)+\boldsymbol{\Theta}_{3}(t) \boldsymbol{r}(t)
$$


where $\boldsymbol{\Theta}_{i}(t)$ are the time-varying estimates of $\boldsymbol{\Theta}_{i}^{*}$ in $(3.3 .11), i=1,2,20,3$, to be updated from some adaptive laws.

Error Model. As in [73], substituting (3.3.14) into (3.3.13) yields

$$
\boldsymbol{\xi}_{m}(s)\left[\boldsymbol{y}-\boldsymbol{y}_{m}\right](t)+\boldsymbol{\Theta}_{0}^{*} \boldsymbol{\xi}_{m}(s)\left[\boldsymbol{y}-\boldsymbol{y}_{m}\right](t)=\boldsymbol{D}_{s} \boldsymbol{S} \tilde{\boldsymbol{\Theta}}^{\top}(t) \boldsymbol{\omega}(t)
$$

where $\boldsymbol{\Theta}^{*}=\left[\boldsymbol{\Theta}_{1}^{* \top}, \boldsymbol{\Theta}_{2}^{* \top}, \Theta_{20}^{*}, \Theta_{3}^{*}\right]^{\top}, \boldsymbol{\Theta}(t)=\left[\boldsymbol{\Theta}_{1}^{\top}(t), \Theta_{2}^{\top}(t), \boldsymbol{\Theta}_{20}(t), \boldsymbol{\Theta}_{3}(t)\right]^{\top}, \tilde{\boldsymbol{\Theta}}(t)=$ $\boldsymbol{\Theta}(t)-\boldsymbol{\Theta}^{*}, \boldsymbol{\omega}(t)=\left[\boldsymbol{\omega}_{1}^{\top}(t), \boldsymbol{\omega}_{2}^{\top}(t), \boldsymbol{y}(t), \boldsymbol{r}(t)\right]^{\top}$, and $\boldsymbol{\Theta}_{0}^{*}=\boldsymbol{L}_{s}^{-1}-\boldsymbol{I}_{M}$ has a special form:

$$
\boldsymbol{\Theta}_{0}^{*}=\left[\begin{array}{ccccc}
0 & 0 & 0 & \ldots & 0 \\
\theta_{21}^{*} & 0 & 0 & \cdots & 0 \\
\theta_{31}^{*} & \theta_{32}^{*} & 0 & \cdots & 0 \\
\vdots & \vdots & \vdots & \vdots & \vdots \\
\theta_{M-11}^{*} & \vdots & \theta_{M-1 M-2}^{*} & 0 & 0 \\
\theta_{M 1}^{*} & 0 & \theta_{M M-2}^{*} & \theta_{M M-1}^{*} & 0
\end{array}\right]
$$

Denote the parameter vectors consisting of the nonzero parameters in each row of $\boldsymbol{\Theta}_{0}^{*}$ as $\boldsymbol{\theta}_{i}^{*}=\left[\theta_{i 1}^{*}, \ldots, \theta_{i i-1}^{*}\right]^{\top} \in \mathbb{R}^{i-1}$, and their estimates as $\boldsymbol{\theta}_{i}(t)=\left[\theta_{i 1}(t), \ldots, \theta_{i i-1}(t)\right]^{\top} \in$ $\mathbb{R}^{i-1}, i=2,3, \ldots, M$. Introduce the estimation error as

$$
\boldsymbol{\epsilon}(t)=\overline{\boldsymbol{e}}(t)+\left[0, \boldsymbol{\theta}_{2}^{\top}(t) \boldsymbol{\eta}_{2}(t), \ldots, \boldsymbol{\theta}_{M}^{\top}(t) \boldsymbol{\eta}_{M}(t)\right]^{\top}+\boldsymbol{\Psi}(t) \boldsymbol{\xi}(t)
$$

where $\overline{\boldsymbol{e}}(t)=\boldsymbol{\xi}_{m}(s) \frac{1}{f(s)}\left[\boldsymbol{y}-\boldsymbol{y}_{m}\right](t)=\left[\bar{e}_{1}(t), \ldots, \bar{e}_{M}(t)\right]^{\top}$ with $f(s)$ being a chosen stable monic polynomial of the same degree as the maximum degree of $\boldsymbol{\xi}_{m}(s), \boldsymbol{\eta}_{i}(t)=$ $\left[\bar{e}_{1}(t), \ldots, \bar{e}_{i-1}(t)\right]^{\top}, i=2,3, \ldots, M, \boldsymbol{\Psi}(t)$ is the estimate of $\boldsymbol{\Psi}^{*}=\boldsymbol{D}_{s} \boldsymbol{S}$, and $\boldsymbol{\xi}(t)=$ $\boldsymbol{\Theta}^{\top}(t) \boldsymbol{\zeta}(t)-h(s)\left[\boldsymbol{\Theta}^{\top} \boldsymbol{\omega}\right](t)$ with $\boldsymbol{\zeta}(t)=h(s)[\boldsymbol{\omega}](t)$. The following error equation is obtained

$$
\boldsymbol{\epsilon}(t)=\left[0, \tilde{\boldsymbol{\theta}}_{2}^{\top}(t) \boldsymbol{\eta}_{2}(t), \ldots, \tilde{\boldsymbol{\theta}}_{M}^{\top}(t) \boldsymbol{\eta}_{M}(t)\right]^{\top}+\boldsymbol{D}_{s} \boldsymbol{S} \tilde{\boldsymbol{\Theta}}^{\top}(t) \boldsymbol{\zeta}(t)+\tilde{\boldsymbol{\Psi}}(t) \boldsymbol{\xi}(t)
$$

with $\tilde{\boldsymbol{\theta}}_{i}(t)=\boldsymbol{\theta}_{i}(t)-\boldsymbol{\theta}_{i}^{*}$ and $\tilde{\boldsymbol{\Psi}}(t)=\boldsymbol{\Psi}(t)-\boldsymbol{\Psi}^{*}$. 
Adaptive Law. Based on the error model (3.3.15), the following gradient adaptive laws are chosen to update $\boldsymbol{\theta}_{i}(t), \boldsymbol{\Theta}(t)$, and $\boldsymbol{\Psi}(t)$ :

$$
\begin{aligned}
\dot{\boldsymbol{\theta}}_{i}(t) & =-\frac{\boldsymbol{\Gamma}_{\theta i} \epsilon_{i}(t) \boldsymbol{\eta}_{i}(t)}{m^{2}(t)}, \quad \boldsymbol{\Gamma}_{\theta i}=\boldsymbol{\Gamma}_{\theta i}^{\boldsymbol{\top}}>\mathbf{0}, \quad i=2,3, \ldots, M \\
\dot{\boldsymbol{\Theta}}^{\top}(t) & =-\frac{\boldsymbol{D}_{s} \boldsymbol{\epsilon}(t) \boldsymbol{\zeta}^{\top}(t)}{m^{2}(t)} \\
\dot{\boldsymbol{\Psi}}(t) & =-\frac{\boldsymbol{\Gamma} \boldsymbol{\epsilon}(t) \boldsymbol{\xi}^{\top}(t)}{m^{2}(t)}, \quad \boldsymbol{\Gamma}=\boldsymbol{\Gamma}^{\boldsymbol{\top}}>\mathbf{0}
\end{aligned}
$$

where $\boldsymbol{\epsilon}(t)=\left[\epsilon_{1}(t), \epsilon_{2}(t), \ldots, \epsilon_{M}(t)\right]^{\top}$ and $m^{2}(t)$ is the normalizing signal

$$
m^{2}(t)=1+\boldsymbol{\zeta}^{\boldsymbol{\top}}(t) \boldsymbol{\zeta}(t)+\boldsymbol{\xi}^{\boldsymbol{\top}}(t) \boldsymbol{\xi}(t)+\sum_{i=2}^{M} \boldsymbol{\eta}_{i}^{\boldsymbol{\top}}(t) \boldsymbol{\eta}_{i}(t)
$$

For $\boldsymbol{K}=\boldsymbol{I}_{M}$, the adaptive controller (3.3.14) with the adaptive laws (3.3.16) ensures closed-loop signal boundedness and asymptotic output tracking [15], i.e.,

$$
\lim _{t \rightarrow \infty}\left(\boldsymbol{y}(t)-\boldsymbol{y}_{m}(t)\right)=\mathbf{0}
$$

Gain Margin Analysis. The desired closed-loop properties of signal boundedness and asymptotic output tracking hold for $\boldsymbol{K}=\operatorname{diag}\left\{k_{1}, k_{2}, \ldots, k_{M}\right\} \neq \boldsymbol{I}_{M}$ with $k_{i}>0$ being constant; that is, we have the gain margin result:

Proposition 3.3.3. A continuous-time multivariable direct MRAC system, designed based on the LDS decomposition of $\boldsymbol{K}_{p}$, has gain margins $(0,+\infty)$ for $k_{i}$ of the input control gain variation matrix $\boldsymbol{K}$ in (3.1.2).

Proof: To prove that the closed-loop system with the adaptive controller designed for $\boldsymbol{K}=\boldsymbol{I}_{M}$ retains the desired performance for $\boldsymbol{K} \neq \boldsymbol{I}_{M}$, we need to prove that the assumptions under which the adaptive controller is designed for $\boldsymbol{K}=\boldsymbol{I}_{M}$ are satisfied.

Since $k_{i}>0$, from $(3.2 .2)$, we have $\bar{\Delta}_{i} \neq 0$, and $\operatorname{sign}\left[\bar{\Delta}_{i}\right]=\operatorname{sign}\left[\Delta_{i}\right]$; that is, the presence of $\boldsymbol{K}$ does not violate the assumptions of nonzero leading principle 
minors and the knowledge of their signs of the high frequency gain matrix, based on which the MRAC scheme is designed for $\boldsymbol{K}=\boldsymbol{I}_{M}$. To be precise, the design parameter $\boldsymbol{D}_{s}$ in (3.3.16) is not affected by a gain matrix $\boldsymbol{K} \neq \boldsymbol{I}_{M}$. Therefore, for $k_{i} \in(0,+\infty), i=1, \ldots, M$, the controller (3.3.14) with the adaptive laws (3.3.16) still ensures the desired system performance, and the MRAC system has gain margin $(0,+\infty)$

Remark 3.3.4. The conclusion in Proposition 3.3.3 reduces to the SISO case when $M=1$ (see Proposition 3.3.2); that is, a continuous-time SISO direct MRAC system has gain margin $(0,+\infty)$.

\subsubsection{Extensions to Other Adaptive Control Systems}

A similar analysis procedure can be applied to other continuous-time direct adaptive control schemes such as state feedback MRAC and nonlinear adaptive control, for either state tracking or output tracking.

State Feedback Designs. For state feedback MRAC designs, the system state variables are used for feedback control and the controlled system (3.1.1) is in the state-space form

$$
\begin{aligned}
& \dot{\boldsymbol{x}}(t)=\boldsymbol{A} \boldsymbol{x}(t)+\boldsymbol{B} \boldsymbol{u}(t) \\
& \boldsymbol{y}(t)=\boldsymbol{C} \boldsymbol{x}(t)
\end{aligned}
$$

with unknown parameter matrices $\boldsymbol{A} \in \mathbb{R}^{n \times n}, \boldsymbol{B} \in \mathbb{R}^{n \times M}$ and $\boldsymbol{C} \in \mathbb{R}^{M \times n}$, and the state vector $\boldsymbol{x}(t) \in \mathbb{R}^{n}$ is available for measurement. The state feedback controller structure is

$$
\boldsymbol{u}(t)=\boldsymbol{K}_{1}^{\top}(t) \boldsymbol{x}(t)+\boldsymbol{K}_{2}(t) \boldsymbol{r}(t)
$$

where $\boldsymbol{K}_{1}(t) \in \mathbb{R}^{n \times M}$ and $\boldsymbol{K}_{2}(t) \in \mathbb{R}^{M \times M}$ are updated from some adaptive laws.

For a state tracking MRAC design, the system state vector signal $\boldsymbol{x}(t)$ is to be controlled to asymptotically track a reference signal $\boldsymbol{x}_{m}(t)$ generated from a chosen 
reference system

$$
\dot{\boldsymbol{x}}_{m}(t)=\boldsymbol{A}_{m} \boldsymbol{x}_{m}(t)+\boldsymbol{B}_{m} \boldsymbol{r}(t)
$$

with $\boldsymbol{A}_{m} \in \mathbb{R}^{n \times n}$ being stable and $\boldsymbol{B}_{m} \in \mathbb{R}^{n \times M}$. Under the restrictive matching conditions that

$$
\boldsymbol{A}+\boldsymbol{B} \boldsymbol{K}_{1}^{* \top}=\boldsymbol{A}_{m}, \quad \boldsymbol{B} \boldsymbol{K}_{2}^{*}=\boldsymbol{B}_{m}
$$

for some constant matrices $\boldsymbol{K}_{1}^{*} \in \mathbb{R}^{n \times M}$ and $\boldsymbol{K}_{2}^{*} \in \mathbb{R}^{M \times M}$, such an adaptive control problem can be solved [15].

For an output tracking design [75], the matching conditions are relaxed to

$$
\boldsymbol{C}\left(s \boldsymbol{I}-\boldsymbol{A}-\boldsymbol{B} \boldsymbol{K}_{1}^{* \boldsymbol{T}}\right)^{-1} \boldsymbol{B} \boldsymbol{K}_{2}^{*}=\boldsymbol{W}_{m}(s), \quad \boldsymbol{K}_{2}^{*-1}=\boldsymbol{K}_{p}
$$

for some constant matrices $\boldsymbol{K}_{1}^{*} \in \mathbb{R}^{n \times M}$ and $\boldsymbol{K}_{2}^{*} \in \mathbb{R}^{M \times M}$, which can be satisfied under the same assumptions as those needed for the output feedback MRAC design. It can be seen that a state feedback for output tracking MRAC design has a much simpler controller structure than the output feedback controller structure (3.3.14).

It can also be verified that the gain margin result of Proposition 3.3.3 holds for the mentioned state feedback MRAC designs as well (for either state or output tracking); that is, the GM is $(0,+\infty)$ for such MRAC systems.

Nonlinear Feedback Designs for Nonlinear Systems. Furthermore, the above gain margin results can be extended to adaptive nonlinear control systems with the controlled systems being nonlinear:

$$
\begin{aligned}
& \dot{\boldsymbol{x}}=\boldsymbol{f}(\boldsymbol{x})+\boldsymbol{g}(\boldsymbol{x}) \boldsymbol{u} \\
& \boldsymbol{y}=\boldsymbol{h}(\boldsymbol{x})
\end{aligned}
$$

Some popular adaptive nonlinear control designs have been developed over the past years, such as those using feedback linearization [74] or backstepping [13], for state tracking or output tracking. Such adaptive control systems can also handle parameter 
uncertainties including control gain variations, to ensure the desired closed-loop system stability and tracking properties in the presence of a positive gain $k$ (or a positive definite diagonal gain matrix $\boldsymbol{K}$ for some special multivariable cases) at input; that is, their gain margin is $(0,+\infty)$.

Remark 3.3.5. If a parameter projection algorithm using the knowledge of either an upper bound or a lower bound of the plant high frequency gain matrix $\boldsymbol{K}_{p}$, then the GM has either an upper bound or a lower bound, different from the above GM results derived for MRAC systems designed using an output error method, in which such an upper or lower bound of $\boldsymbol{K}_{p}$ is not used. For a MRAC system designed using an input error method [10,76], an upper bound of $\boldsymbol{K}_{p}$ is used, while for a prediction model based adaptive control system [77] (developed mainly in discrete time; see next subsection), a lower bound of $\boldsymbol{K}_{p}$ is used, with an advantage that both have less parameters to estimate. This clarification also applies to nonlinear adaptive control systems.

While the above GM results (that the GM is $(0,+\infty)$ for continuous-time adaptive control systems) are understandable in the sense that MRAC can handle large parameter uncertainties, the multivariable GM results are derived based on Lemma 3.2.1 (for multivariable nonlinear dynamic systems, adaptive control designs have not been developed as thoroughly as that for multivariable linear systems, so that more work is needed for adaptive control of multivariable nonlinear systems). We should note that the GM analysis of discrete-time MRAC systems is more involved than that for continuous-time systems, as shown next. 


\subsection{Gain Margins of Discrete-Time MRAC Sys- tems}

Discrete-time multivariable MRAC shares the same controller structure and matching conditions as its continuous-time counterpart. However, it has different stability characterization, leading to different signal filters, adaptation gains, design conditions, as well as stability and robust analysis. For a comparison with the GM analysis of the continuous-time design, we present the GM result for the discrete-time MRAC

design based on the LDS decomposition of $\boldsymbol{K}_{p}$, in the presence of a diagonal gain matrix $\boldsymbol{K}$ at the control input.

\subsubsection{Design for SISO Systems}

The discrete-time plant model is (3.1.1) with $D$ replaced by $z$, i.e.,

$$
y(t)=G(z)[u](t), \quad G(z)=k_{p} \frac{Z(z)}{P(z)}
$$

where $k_{p}$ is the plant high frequency gain, $y(t) \in \mathbb{R}$ is the plant output, $u(t) \in \mathbb{R}$ is the plant input, and

$$
Z(z)=z^{m}+z_{m-1} z^{m-1}+\cdots+z_{1} z+z_{0}, \quad P(z)=z^{n}+p_{n-1} z^{n-1}+\cdots+p_{1} z+p_{0}
$$

are polynomials in $z$ with $z_{i}, i=0,1, \ldots, m-1$, and $p_{j}, j=0,1, \ldots, n-1, n>m$, being unknown but constant parameters.

MRAC Assumptions. For discrete-time SISO MRAC designs, the Assumptions (A3.1-1)-(A3.1-3) and (A3.1-4D) reduce to:

(A3.4-1) $Z(z)$ is a stable polynomial;

(A3.4-2) The degree $n$ of $P(z)$ is known;

(A3.4-3) The relative degree $n^{*}=n-m>0$ is known; and 
(A3.4-4) The sign of $k_{p}$ is known, and $\left|k_{p}\right| \leq k_{p}^{0}$ for some constant $k_{p}^{0}>0$.

The reference model transfer function in (3.1.3) is chosen as $W_{m}(z)=z^{-n^{*}}$, such that $y_{m}\left(t+n^{*}\right)=r(t)$.

Controller Structure. The following adaptive control law is applied:

$$
u(t)=\boldsymbol{\theta}_{1}^{\top}(t) \boldsymbol{\omega}_{1}(t)+\boldsymbol{\theta}_{2}^{\top}(t) \boldsymbol{\omega}_{2}(t)+\theta_{3}(t) y_{m}\left(t+n^{*}\right)
$$

where $\boldsymbol{\theta}_{1}(t) \in \mathbb{R}^{n-1}, \boldsymbol{\theta}_{2}(t) \in \mathbb{R}^{n}, \theta_{3}(t) \in \mathbb{R}$ are controller parameters to be updated from an adaptive law, and the filtered vector signals are defined as

$$
\boldsymbol{\omega}_{1}(t)=\boldsymbol{a}_{\lambda}(z)[u](t), \quad \boldsymbol{\omega}_{2}(t)=\boldsymbol{b}_{\lambda}(z)[y](t)
$$

with $\boldsymbol{a}_{\lambda}(z)=\left[z^{-n+1}, \ldots, z^{-1}\right]^{\top}$, and $\boldsymbol{b}_{\lambda}(z)=\left[a_{\lambda}^{\top}(z), 1\right]^{\top}$.

If the plant parameters are known, the control objective can be achieved by implementing the control law (3.4.2) with $\boldsymbol{\theta}_{i}(t)=\boldsymbol{\theta}_{i}^{*}, i=1,2,3$, where $\boldsymbol{\theta}_{1}^{*}$ and $\boldsymbol{\theta}_{2}^{*}$ are computed from the plant-model matching equation:

$$
\theta_{1}^{* \top} \boldsymbol{a}_{\lambda}(z) P(z)+\theta_{2}^{* \top} \boldsymbol{b}_{\lambda}(z) k_{p} Z(z)=P(z)-Z(z) z^{n^{*}}, \quad \theta_{3}^{*}=k_{p}^{-1}
$$

This implementation ensures closed-loop signal boundedness and exact output tracking after $n^{*}$ steps, i.e., $y\left(t+n^{*}\right)-y_{m}\left(t+n^{*}\right)=0$ for $t=0,1,2, \ldots$.

For plant with unknown parameters, adaptive laws are developed to update the time-varying estimates of $\boldsymbol{\theta}_{i}^{*}, \boldsymbol{\theta}_{i}(t), i=1,2,3$. Multiplying both sides of (3.4.3) by $\frac{1}{P(z)}$, and applying the equation on $u(t)$ yields

$$
u(t)=\boldsymbol{\theta}_{1}^{* \mathrm{~T}} \boldsymbol{\omega}_{1}(t)+\boldsymbol{\theta}_{2}^{* \mathrm{~T}} \boldsymbol{\omega}_{2}(t)+\theta_{3}^{*} y\left(t+n^{*}\right)
$$

By introducing $\rho^{*}=k_{p}, \boldsymbol{\omega}(t)=\left[\boldsymbol{\omega}_{1}^{\top}(t), \boldsymbol{\omega}_{2}^{\top}(t), y_{m}\left(t+n^{*}\right)\right]^{\top}, \boldsymbol{\theta}(t)=\left[\boldsymbol{\theta}_{1}^{\top}(t), \boldsymbol{\theta}_{2}^{\top}(t), \theta_{3}(t)\right]^{\top}$, $\boldsymbol{\theta}^{*}=\left[\boldsymbol{\theta}_{1}^{* \top}, \boldsymbol{\theta}_{2}^{* \top}, \theta_{3}^{*}\right]^{\top}, e(t)=y(t)-y_{m}(t), \tilde{\boldsymbol{\theta}}(t)=\boldsymbol{\theta}(t)-\boldsymbol{\theta}^{*}$, and substituting (3.4.4) into (3.4.2), the tracking error equation follows

$$
e(t)=-\rho^{*}\left(\boldsymbol{\theta}^{* \boldsymbol{\top}} \boldsymbol{\omega}\left(t-n^{*}\right)-\boldsymbol{\theta}^{\boldsymbol{\top}}\left(t-n^{*}\right) \boldsymbol{\omega}\left(t-n^{*}\right)\right)
$$


Error Model. Define the estimation error as

$$
\epsilon(t)=e(t)+\rho(t) \xi(t), \quad \xi(t)=\boldsymbol{\theta}^{\top}(t) \boldsymbol{\omega}\left(t-n^{*}\right)-\boldsymbol{\theta}^{\top}\left(t-n^{*}\right) \boldsymbol{\omega}\left(t-n^{*}\right)
$$

where $\rho(t)$ is an estimate of $\rho^{*}$. In view of (3.4.5), we can obtain the error model

$$
\epsilon(t)=\rho^{*} \tilde{\boldsymbol{\theta}}^{\top}(t) \boldsymbol{\omega}\left(t-n^{*}\right)+\tilde{\rho}(t) \xi(t)
$$

where $\tilde{\rho}(t)=\rho(t)-\rho^{*}$.

Adaptive Law. The gradient adaptive laws are applied to update $\boldsymbol{\theta}(t)$ and $\rho(t)$ :

$$
\begin{aligned}
& \boldsymbol{\theta}(t+1)=\boldsymbol{\theta}(t)-\frac{\operatorname{sign}\left[k_{p}\right] \boldsymbol{\Gamma} \epsilon(t) \boldsymbol{\omega}\left(t-n^{*}\right)}{m^{2}(t)}, \quad 0<\boldsymbol{\Gamma}=\boldsymbol{\Gamma}^{\boldsymbol{\top}}<\frac{2}{k_{p}^{0}} \boldsymbol{I}_{2 n} \\
& \rho(t+1)=\rho(t)-\frac{\gamma \epsilon(t) \xi(t)}{m^{2}(t)}, \quad 0<\gamma<2
\end{aligned}
$$

where $m(t)=\sqrt{1+\boldsymbol{\omega}^{\top}\left(t-n^{*}\right) \boldsymbol{\omega}\left(t-n^{*}\right)+\xi^{2}(t)}$.

For $k=1$, the controller (3.4.2) with the adaptive laws (3.4.6) ensures that for any bounded initial conditions, all signals in the closed-loop system are bounded and the output $y(t)$ tracks the reference signal $y_{m}(t)$ asymptotically, i.e., $\lim _{t \rightarrow \infty}(y(t)-$ $\left.y_{m}(t)\right)=0[15]$

Gain Margin Analysis. The same stability properties and asymptotic tracking performance hold for any constant $k$ within a specified range; that is, we have the gain margin result:

Proposition 3.4.1. A discrete-time SISO direct MRAC system has gain margin $\left(0, \frac{k_{p}^{0}}{\left|k_{p}\right|}\right]$, where $\left|k_{p}\right| \leq k_{p}^{0}$ such that $\mathbf{0}<\boldsymbol{\Gamma}=\boldsymbol{\Gamma}^{\boldsymbol{\top}}<\frac{2}{k_{p}^{0}} \boldsymbol{I}_{2 n}$ for the adaptive laws (3.4.6).

Proof: The adaptive laws (3.4.6) are designed for the plant when the constant gain is $k=1$. When $k \neq 1$, the system high frequency gain becomes $k_{p} k, \rho(t)$ becomes the estimate of $\rho^{*}=k_{p} k$, and the controller parameter $\boldsymbol{\theta}(t)$ is adjusted accordingly. 
For $k=1$, the design condition is $\mathbf{0}<\boldsymbol{\Gamma}=\boldsymbol{\Gamma}^{\boldsymbol{\top}}<\frac{2}{k_{p}^{0}} \boldsymbol{I}_{2 n}$, where $\left|k_{p}\right| \leq k_{p}^{0}$, while the stability condition is $\mathbf{0}<\boldsymbol{\Gamma}=\boldsymbol{\Gamma}^{\boldsymbol{\top}}<\frac{2}{\left|k_{p}\right|} \boldsymbol{I}_{2 n}$ which is implied by the design condition. For $k \neq 1$, the adaptive controller (3.4.2) still ensures desired closed-loop system performance if $\left|k_{p} k\right| \leq k_{p}^{0}$, that is, $k \in\left(0, \frac{k_{p}^{0}}{\left|k_{p}\right|}\right]$, because the stability condition now is $\mathbf{0}<\boldsymbol{\Gamma}=\boldsymbol{\Gamma}^{\boldsymbol{\top}}<\frac{2}{\left|k_{p}\right| k} \boldsymbol{I}_{2 n}$ which is still ensured by the design condition. Therefore, the adaptive control system has gain margin $\left(0, \frac{k_{p}^{0}}{\left|k_{p}\right|}\right]$.

Remark 3.4.2. The specified range of the constant gain $k$ in Proposition 3.4 .1 is a sufficient condition for ensuring desired closed-loop system performance; that is, there may exist some $k \in\left(\frac{k_{p}^{0}}{\left|k_{p}\right|},+\infty\right)$ that results in a stable closed-loop system under some initial conditions, whereas as long as $k$ falls into $\left(0, \frac{k_{p}^{0}}{\left|k_{p}\right|}\right]$, the system is stable for any initial conditions and the plant output asymptotically follows a desired trajectory generated by the reference model system.

Remark 3.4.3. With the conclusion that the discrete-time SISO direct MRAC scheme has a finite gain margin, the question how to increase the gain margin is of interest. From Proposition 3.4.1, one way we can increase the gain margin is to increase $k_{p}^{0}$, i.e., in the system design process, assume a higher upper bound on $\left|k_{p}\right|$. However, from the constraint on the adaptive law (3.4.6), the adaptation gain matrix $\boldsymbol{\Gamma}$ has to satisfy $\mathbf{0}<\boldsymbol{\Gamma}=\boldsymbol{\Gamma}^{\boldsymbol{\top}}<\frac{2}{k_{p}^{0}} \boldsymbol{I}_{2 n}$ for closed-loop signal boundedness and output tracking. This constraint will result in a slower adaptation speed for $\boldsymbol{\theta}(t)$. Therefore, to increase the gain margin of the system, we assume a higher $k_{p}^{0}$, while choosing the adaptation gain matrix $\boldsymbol{\Gamma}$ such that $\mathbf{0}<\boldsymbol{\Gamma}=\boldsymbol{\Gamma}^{\boldsymbol{\top}}<\frac{2}{k_{p}^{0}} \boldsymbol{I}_{2 n}$.

\subsubsection{LDS Decomposition Based Design for MIMO Systems}

As described in Section 3.3, for $\boldsymbol{K}=\boldsymbol{I}_{M}$, when the system parameters in $\boldsymbol{P}(z)$ and $\boldsymbol{Z}(z)$, the polynomial $\Lambda(z)$, and the modified interactor matrix $\boldsymbol{\xi}_{m}(z)$ are known, the 
controller (3.3.11) with parameters $\boldsymbol{\Theta}_{i}^{*}, i=1,2,20,3$, computed from the matching equation (3.3.12) with $s=z$, can be applied to achieve the control objective.

Without knowledge of the plant parameters, by following a similar procedure of development as in Section 3.3 with $s=z$, we can obtain the estimation error model (3.3.15), based on which the following gradient adaptive laws are chosen:

$$
\begin{aligned}
\boldsymbol{\theta}_{i}(t+1)-\boldsymbol{\theta}_{i}(t) & =-\frac{\boldsymbol{\Gamma}_{\theta i} \epsilon_{i}(t) \boldsymbol{\eta}_{i}(t)}{m^{2}(t)}, i=2, \ldots, M \\
\boldsymbol{\Theta}^{\top}(t+1)-\boldsymbol{\Theta}^{\top}(t) & =\frac{\boldsymbol{D}_{s} \boldsymbol{\epsilon}(t) \boldsymbol{\zeta}^{\top}(t)}{m^{2}(t)} \\
\boldsymbol{\Psi}(t+1)-\boldsymbol{\Psi}(t) & =-\frac{\boldsymbol{\Gamma} \boldsymbol{\epsilon}(t) \boldsymbol{\xi}^{\top}(t)}{m^{2}(t)}
\end{aligned}
$$

where $\mathbf{0}<\boldsymbol{\Gamma}_{\theta i}=\boldsymbol{\Gamma}_{\theta i}^{\top}<2 \boldsymbol{I}_{i-1}, \mathbf{0}<\boldsymbol{\Gamma}=\boldsymbol{\Gamma}^{\top}<2 \boldsymbol{I}_{M}$, and

$$
\boldsymbol{D}_{s}=\operatorname{diag}\left\{\operatorname{sign}\left[\Delta_{1}\right] \gamma_{1}, \operatorname{sign}\left[\frac{\Delta_{2}}{\Delta_{1}}\right] \gamma_{2}, \ldots, \operatorname{sign}\left[\frac{\Delta_{M}}{\Delta_{M-1}}\right] \gamma_{M}\right\}
$$

with $\gamma_{i}>0$, is chosen to satisfy

$$
\mathbf{0}<\boldsymbol{D}_{s} \boldsymbol{U}^{\top} \boldsymbol{D}_{s}^{-1} \boldsymbol{D}^{*} \boldsymbol{U} \boldsymbol{D}_{s}<2 \boldsymbol{I}_{M}
$$

that is, $\gamma_{i} \in\left(0, \gamma_{i}^{0}\right)$ for some $\gamma_{i}^{0}>0, i=1,2, \ldots, M$.

Remark 3.4.4. The choice of the design parameters $\gamma_{i}$ is not straightforward from (3.4.8). To proceed, we propose a numerical method. The adaptation gain matrix $\boldsymbol{D}_{s}$ in (3.4.7) is first decomposed as $\boldsymbol{D}_{s}=\boldsymbol{L}_{d} \boldsymbol{D}^{*}$ for some positive definite and diagonal matrix $\boldsymbol{L}_{d}$. Then (3.4.8) is equivalent to

$$
\mathbf{0}<\boldsymbol{L}_{d} \boldsymbol{D}^{*} \boldsymbol{U}^{\top} \boldsymbol{L}_{d}^{-1} \boldsymbol{U} \boldsymbol{L}_{d} \boldsymbol{D}^{*}<2 \boldsymbol{I}_{M}
$$

which by Schur complement results in

$$
\left[\begin{array}{cc}
2 \boldsymbol{I}_{M} & \boldsymbol{L}_{d} \boldsymbol{D}^{*} \boldsymbol{U}^{\top} \\
\boldsymbol{U} \boldsymbol{D}^{*} \boldsymbol{L}_{d} & \boldsymbol{L}_{d}
\end{array}\right]>\mathbf{0}
$$


Thus the problem is converted to the solution of an LMI problem for a diagonal matrix $\boldsymbol{L}_{d}$, which can be handled numerically, e.g., by MATLAB. Note that knowledge of $\boldsymbol{D}^{*}$ and $\boldsymbol{U}$ are required.

If $\gamma_{i}^{0}$ is needed for a choice of $\gamma_{i}$ such that $\gamma_{i} \in\left(0, \gamma_{i}^{0}\right)$, a linear cost function can be constructed and optimized subject to the LMI constraints (3.4.9) and $\boldsymbol{L}_{d}>\mathbf{0}$.

For $\boldsymbol{K}=\boldsymbol{I}_{M}$, the adaptive control law (3.3.14) with the adaptive laws (3.4.7) ensures closed-loop signal boundedness and asymptotic output tracking [15], i.e.,

$$
\lim _{t \rightarrow \infty}\left(\boldsymbol{y}(t)-\boldsymbol{y}_{m}(t)\right)=\mathbf{0}
$$

Gain Margin Analysis. In the presence of $\boldsymbol{K}=\operatorname{diag}\left\{k_{1}, k_{2}, \ldots, k_{M}\right\} \neq \boldsymbol{I}_{M}$ with $k_{i}>0$ being constant, the same desired closed-loop system performance holds if $k_{i} \leq k_{i}^{0}$ for some $k_{i}^{0}>0$; that is, we have the gain margin result:

Proposition 3.4.5. A discrete-time multivariable direct MRAC system, designed based on the LDS decomposition of $\boldsymbol{K}_{p}$, has gain margins $\left(0, k_{i}^{0}\right]$ for $k_{i}$ of the input control gain variation matrix $\boldsymbol{K}=\operatorname{diag}\left\{k_{1}, k_{2}, \ldots, k_{M}\right\}$, where $\boldsymbol{K}^{0}=\operatorname{diag}\left\{k_{1}^{0}, k_{2}^{0}, \ldots, k_{M}^{0}\right\}$ satisfies

$$
\mathbf{0}<\boldsymbol{D}_{s} \boldsymbol{K}^{0} \boldsymbol{U}^{\top}\left(\boldsymbol{K}^{0}\right)^{-1} \boldsymbol{D}_{s}^{-1} \boldsymbol{D}^{*} \boldsymbol{U} \boldsymbol{K}^{0} \boldsymbol{D}_{s}<2 \boldsymbol{I}_{M}
$$

for $\boldsymbol{D}_{\text {s }}$ chosen to meet the condition (3.4.8).

Proof: From Lemma 3.2.1, the presence of a gain matrix $\boldsymbol{K} \neq \boldsymbol{I}_{M}$ leads to the new high frequency gain matrix $\boldsymbol{K}_{p} \boldsymbol{K}$ with its LDU decomposition as $\boldsymbol{K}_{p} \boldsymbol{K}=\overline{\boldsymbol{L}} \overline{\boldsymbol{D}}^{*} \overline{\boldsymbol{U}}$ for $\overline{\boldsymbol{D}}^{*}=\boldsymbol{D}^{*} \boldsymbol{K}$ from the LDU decomposition of $\boldsymbol{K}_{p}, \boldsymbol{K}_{p}=\boldsymbol{L} \boldsymbol{D}^{*} \boldsymbol{U}$. We can see that the sign information of the leading principle minors of $\boldsymbol{K}_{p} \boldsymbol{K}$ is the same as that of $\boldsymbol{K}_{p}$ so that the adaptive law with $\boldsymbol{D}_{s}$ chosen for $\boldsymbol{K}=\boldsymbol{I}_{M}$ can still be used for $\boldsymbol{K} \neq \boldsymbol{I}_{M}$.

However, to ensure the closed-loop signal boundedness and asymptotic output tracking, the new condition for $\boldsymbol{K} \neq \boldsymbol{I}_{M}$, similar to (3.4.8) for $\boldsymbol{K}=\boldsymbol{I}_{M}$, is $\mathbf{0}<$ 
$\boldsymbol{D}_{s} \overline{\boldsymbol{U}}^{\top} \boldsymbol{D}_{s}^{-1} \overline{\boldsymbol{D}}^{*} \overline{\boldsymbol{U}} \boldsymbol{D}_{s}<2 \boldsymbol{I}_{M}$, which needs to be satisfied for the chosen $\boldsymbol{D}_{s}$ for (3.4.8). From Lemma 3.2.1, this condition is equivalent to

$$
\mathbf{0}<\boldsymbol{D}_{s} \boldsymbol{K} \boldsymbol{U}^{\top} \boldsymbol{K}^{-1} \boldsymbol{D}_{s}^{-1} \boldsymbol{D}^{*} \boldsymbol{U} \boldsymbol{K} \boldsymbol{D}_{s}<2 \boldsymbol{I}_{M}
$$

It can be seen that for $k_{i}$, there is an upper bound $k_{i}^{0}>0$, which depends on $\gamma_{j}$, $k_{j}, j=1,2, \ldots, i-1, d_{j}^{*}, d_{j}^{0}, j=1,2, \ldots, i$, and the nonzero elements of $\boldsymbol{U}$, with $\boldsymbol{K}^{0}=\operatorname{diag}\left\{k_{1}^{0}, k_{2}^{0}, \ldots, k_{M}^{0}\right\}$ satisfying (3.4.10) such that the closed-loop performance is still achieved with the MRAC design for $\boldsymbol{K}=\boldsymbol{I}_{M}$. In other words, the gain margins are $\left(0, k_{i}^{0}\right], i=1,2, \ldots, M$.

It is desirable to have an explicit expression for $\boldsymbol{K}^{0}$ in (3.4.10). The results for $M=2$ are presented later in this subsection. For a more general case, such an explicit solution may not exist because of the coupling of $\gamma_{i}$ with each other and the unknown form of $\boldsymbol{U}$. A numerical method is proposed as follows.

With $\boldsymbol{D}_{\boldsymbol{s}}$ chosen to satisfy (3.4.8), to derive the GM, we need to solve (3.4.11) for a range of $\boldsymbol{K}$. With $\boldsymbol{D}_{s}=\boldsymbol{L}_{d} \boldsymbol{D}^{*}$ for some $\boldsymbol{L}_{d}>0$ and by the Schur Complement, (3.4.11) is equivalent to

$$
\left[\begin{array}{cc}
2 \boldsymbol{I}_{M} & \boldsymbol{L}_{d} \boldsymbol{D}^{*} \boldsymbol{K} \boldsymbol{U}^{\top} \\
\boldsymbol{U} \boldsymbol{K} \boldsymbol{D}^{*} \boldsymbol{L}_{d} & \boldsymbol{K} \boldsymbol{L}_{d}
\end{array}\right]>\mathbf{0}
$$

We can then construct a linear cost function, and optimize it subject to the LMI constraints (3.4.12) and $\boldsymbol{K}>0$. The range of $\boldsymbol{K}$ can thus be obtained.

Remark 3.4.6. The gain margin result in Proposition 3.4 .5 reduces to the SISO case when $M=1$, that is, a discrete-time SISO direct MRAC system has gain margin $\left(0, \frac{k_{p}^{0}}{\left|k_{p}\right|}\right]$, where $k_{p}^{0}$ is known such that $0<\left|k_{p}\right| \leq k_{p}^{0}$ (see Proposition 3.4.1). This result can be shown briefly as follows.

For $M=1$, the high frequency gain matrix $\boldsymbol{K}_{p}$ becomes a scalar $k_{p}, \boldsymbol{D}^{*}=k_{p}$, $\boldsymbol{L}=\boldsymbol{U}=1$, and $\boldsymbol{D}_{s}=\operatorname{sign}\left[k_{p}\right] \gamma$. The design condition (3.4.8) yields $0<\gamma<$ 
$\frac{2}{\operatorname{sign}\left[k_{p}\right] k_{p}}=\frac{2}{\left|k_{p}\right|}$, which is satisfied if $0<\gamma<\frac{2}{k_{p}^{0}}$. When the gain $\boldsymbol{K}=k \neq 1$, due to the fact $\overline{\boldsymbol{D}}^{*}=k_{p} k$ and $\operatorname{sign}[\bar{\Delta}]=\operatorname{sign}\left[k_{p}\right]$, it follows that (3.4.11) yields $0<k\left|k_{p}\right| \gamma<2$, which is satisfied if $0<k\left|k_{p}\right| \gamma<k\left|k_{p}\right| \frac{2}{k_{p}^{0}} \leq 2$, from which we obtain the gain margin $\left(0, \frac{k_{p}^{0}}{\left|k_{p}\right|}\right]$ for SISO direct MRAC systems.

Remark 3.4.7. When $\boldsymbol{K}_{p}$ is lower triangular, from its LDU decomposition, $\boldsymbol{K}_{p}=$ $\boldsymbol{L} \boldsymbol{D}^{*} \boldsymbol{U}$, we have $\boldsymbol{U}=\boldsymbol{I}_{M}$, and (3.4.8) is equivalent to $\mathbf{0}<\boldsymbol{D}^{*} \boldsymbol{D}_{s}<2 \boldsymbol{I}_{M}$, that is, $\mathbf{0}<\operatorname{diag}\left\{\left|d_{1}^{*}\right| \gamma_{1},\left|d_{2}^{*}\right| \gamma_{2}, \ldots,\left|d_{M}^{*}\right| \gamma_{M}\right\}<2 \boldsymbol{I}_{M}$. It is satisfied if

$$
\operatorname{diag}\left\{\gamma_{1}, \gamma_{2}, \ldots, \gamma_{M}\right\}<\operatorname{diag}\left\{\frac{2}{d_{1}^{0}}, \frac{2}{d_{2}^{0}}, \ldots, \frac{2}{d_{M}^{0}}\right\}
$$

For $\boldsymbol{K} \neq \boldsymbol{I}_{M}$, the stability condition in (3.4.11) yields

$$
\mathbf{0}<\operatorname{diag}\left\{\left|d_{1}^{*}\right| k_{1} \gamma_{1},\left|d_{2}^{*}\right| k_{2} \gamma_{2}, \ldots,\left|d_{M}^{*}\right| k_{M} \gamma_{M}\right\}<2 \boldsymbol{I}_{M}
$$

which, from (3.4.13), is satisfied if

$$
\mathbf{0}<\operatorname{diag}\left\{k_{1}, k_{2}, \ldots, k_{M}\right\} \leq \operatorname{diag}\left\{\frac{d_{1}^{0}}{\left|d_{1}^{*}\right|}, \frac{d_{2}^{0}}{\left|d_{2}^{*}\right|}, \ldots, \frac{d_{M}^{0}}{\left|d_{M}^{*}\right|}\right\}
$$

Therefore, we have the gain margin result

$$
k_{i} \in\left(0, \frac{d_{i}^{0}}{\left|d_{i}^{*}\right|}\right], \quad i=1,2, \ldots, M,
$$

where $d_{i}^{0}, i=1,2, \ldots, M$, are from Assumption (A3.1-4D).

Remark 3.4.8. For a discrete-time direct $M R A C$ system, the $G M$ is $\left(0, k_{i}^{0}\right]$, where $k_{i}^{0}$ can be made large by reducing the adaptation gain. This can been seen from (2.2.11) and (3.4.11). As $\boldsymbol{D}^{*}$ and $\boldsymbol{U}$ are constant matrices, we can increase $k_{i}^{0}$ by reducing the design parameters $\gamma_{i}>0$, which reduces the adaptation speed for $\boldsymbol{\Theta}(t)$ in (3.4.7). Therefore, the GM can be enlarged by assuming larger $d_{i}^{0}, i=1,2, \ldots, M$, in the system design process, while at the same time maintaining the inequality in (3.4.11). This becomes straightforward for the case when $\boldsymbol{U}=\boldsymbol{I}_{M}$. 
The same results can be derived for a discrete-time version of the state feedback for output tracking MRAC system [75]; that is, the GM is: $k_{i} \in\left(0, k_{i}^{0}\right]$ for some $k_{i}^{0}>0$. For the SISO case, its GM is finite with a finite upper bound, i.e., $\left(0, \frac{k_{p}^{0}}{\left|k_{p}\right|}\right]$, where $k_{p}^{0}$ is the known upper bound of $\left|k_{p}\right|$. An input error MRAC design can also be derived for a discrete-time multivariable LTI system. For such an adaptive control system, some upper bound information of $\boldsymbol{K}_{p}$ is used, so that the GM also has an upper bound which directly depends on the upper bound used in parameter projection for the adaptive law. A prediction model based adaptive control design [77] uses a lower bound of $\boldsymbol{K}_{p}$, and its GM has a lower bound (instead of an upper bound for the above output error method based MRAC systems).

\subsubsection{Gain Margin Bounds for $M=2$}

The explicit GM bounds for $M=2$ are provided here as a demonstration of the GM result in Proposition 3.4.5, and the derivation is included in Section 9.1.

Denote the $(1,2)$ element of the $2 \times 2$ unity upper triangular matrix $\boldsymbol{U}$ as $a$. For $a=0$, the design parameters $\gamma_{1}$ and $\gamma_{2}$ must be chosen to satisfy

$$
0<\gamma_{1}<\frac{2}{d_{1}^{0}}, \quad 0<\gamma_{2}<\frac{2}{d_{2}^{0}}
$$

For $a \neq 0$, the same range for $\gamma_{1}$ in (3.4.15) holds, and

$$
0<\gamma_{2}<\frac{\alpha\left(\gamma_{1}\right) d_{2}^{0}+\sqrt{\alpha\left(\gamma_{1}\right) \beta\left(\gamma_{1}\right)}}{4 a^{2} d_{1}^{0}}
$$

where $d_{1}^{0}$ and $d_{2}^{0}$, assumed to be known, are the upper bounds of $\left|d_{1}^{*}\right|$ and $\left|d_{2}^{*}\right|$, and $\alpha\left(\gamma_{1}\right)=\gamma_{1}\left(d_{1}^{0} \gamma_{1}-2\right), \beta\left(\gamma_{1}\right)=\alpha\left(\gamma_{1}\right)\left(d_{2}^{0}\right)^{2}-16 a^{2} d_{1}^{0}$.

The gain margin results for $a=0$ are:

$$
0<k_{1} \leq \frac{d_{1}^{0}}{\left|d_{1}^{*}\right|}, \quad 0<k_{2} \leq \frac{d_{2}^{0}}{\left|d_{2}^{*}\right|}
$$


For $a \neq 0$, the same range for $k_{1}$ in (3.4.17) holds, and

$$
0<k_{2} \leq \frac{d_{1}^{0}}{\left|d_{1}^{*}\right|} \frac{\bar{\alpha}\left(k_{1} \gamma_{1}\right)\left|d_{2}^{*}\right|+\sqrt{\bar{\alpha}\left(k_{1} \gamma_{1}\right) \bar{\beta}\left(k_{1} \gamma_{1}\right)}}{\alpha\left(\gamma_{1}\right) d_{2}^{0}+\sqrt{\alpha\left(\gamma_{1}\right) \beta\left(\gamma_{1}\right)}}
$$

where $\bar{\alpha}\left(k_{1} \gamma_{1}\right)=k_{1} \gamma_{1}\left(\left|d_{1}^{*}\right| k_{1} \gamma_{1}-2\right)$, and $\bar{\beta}\left(k_{1} \gamma_{1}\right)=\bar{\alpha}\left(k_{1} \gamma_{1}\right)\left|d_{2}^{*}\right|^{2}-16 a^{2}\left|d_{1}^{*}\right|$ (see Section 9.1 for details).

\subsection{Gain Margins of Indirect MRAC Systems}

Indirect multivariable MRAC schemes are of interest because there are less parameters to be estimated and less filters to be implemented than direct MRAC. Moreover, the system parameters carry more physical meanings than controller parameters, and it is more natural and practical to expect a priori knowledge about system parameters than controller parameters, which further reduces computational burden. Next, we present the design of continuous-time and discrete-time indirect MRAC schemes applied to SISO systems, followed by the GM analysis. A multivariable design for a class of MIMO systems with its GM analysis is presented in a unified framework for both continuous- and discrete-time cases.

\subsubsection{Design for Continuous-Time SISO Systems}

Consider the continuous-time plant model (3.3.6), i.e.,

$$
y(t)=G(s)[u](t), \quad G(s)=k_{p} \frac{Z(s)}{P(s)}
$$

where $k_{p}$ is the plant high frequency gain, $y(t) \in \mathbb{R}$ is the plant output, $u(t) \in \mathbb{R}$ is the plant input, and

$$
Z(s)=s^{m}+z_{m-1} s^{m-1}+\cdots+z_{1} s+z_{0}, \quad P(s)=s^{n}+p_{n-1} s^{n-1}+\cdots+p_{1} s+p_{0}
$$

are polynomials in $s$ with $z_{i}, i=0,1, \ldots, m-1$, and $p_{j}, j=0,1, \ldots, n-1, n>m$, being unknown but constant parameters. It can be parameterized as follows by 
choosing stable polynomials $\Lambda_{e}(s)=s^{n}+\lambda_{n-1}^{e} s^{n-1}+\cdots+\lambda_{1}^{e} s+\lambda_{0}^{e}$ and $\Lambda_{n-1}(s)=$ $\lambda_{n-1}^{e} s^{n-1}+\cdots+\lambda_{1}^{e} s+\lambda_{0}^{e}:$

$$
y(t)-\frac{\Lambda_{n-1}(s)}{\Lambda_{e}(s)}[y](t)=\boldsymbol{\theta}_{p}^{* \top} \boldsymbol{\phi}(t)
$$

where $\boldsymbol{\theta}_{p}^{*}=\left[k_{p} z_{0}, \ldots, k_{p} z_{m-1}, k_{p},-p_{0}, \ldots,-p_{n-1}\right]^{\top}$ is the parameter vector, and

$$
\begin{aligned}
\phi(t)= & {\left[\frac{1}{\Lambda_{e}(s)}[u](t), \frac{s}{\Lambda_{e}(s)}[u](t), \ldots, \frac{s^{m}}{\Lambda_{e}(s)}[u](t),\right.} \\
& \left.\frac{1}{\Lambda_{e}(s)}[y](t), \frac{s}{\Lambda_{e}(s)}[y](t), \ldots, \frac{s^{n-1}}{\Lambda_{e}(s)}[y](t)\right]^{\top}
\end{aligned}
$$

is the regressor vector.

MRAC Assumptions. The indirect MRAC schemes share the same design assumptions as their continuous-time direct MRAC counterparts (see Section 3.3.2) except for

(A3.5-1) The lower bound $k_{p 0}$ of $\left|k_{p}\right|$, such that $0<k_{p 0} \leq\left|k_{p}\right|$, is known, and so is $\operatorname{sign}\left[k_{p}\right]$, the sign of $k_{p}$.

The transfer function of the reference model (3.1.3) is chosen to be $W_{m}(s)=$ $P_{m}^{-1}(s)$ with the monic stable polynomial $P_{m}(s)$ of degree $n-m$.

Error Model and Adaptive Law. Denoting $\boldsymbol{\theta}_{p}(t)$ as the estimate of $\boldsymbol{\theta}_{p}^{*}$, we define the estimation error

$$
\epsilon(t)=\boldsymbol{\theta}_{p}^{\top}(t) \boldsymbol{\phi}(t)-y(t)+\frac{\Lambda_{n-1}(s)}{\Lambda_{e}(s)}[y](t), \quad t \geq t_{0}
$$

based on which the gradient adaptive law with parameter projection is applied to update $\boldsymbol{\theta}_{p}(t)$ :

$$
\dot{\boldsymbol{\theta}}_{p}(t)=-\frac{\boldsymbol{\Gamma} \boldsymbol{\phi}(t) \epsilon(t)}{m^{2}(t)}+\boldsymbol{f}(t), \quad \boldsymbol{\theta}_{p}\left(t_{0}\right)=\boldsymbol{\theta}_{0}, \quad t \geq t_{0}
$$

where $\boldsymbol{\Gamma}=\operatorname{diag}\left\{\boldsymbol{\Gamma}_{1}, \gamma_{m+1}, \boldsymbol{\Gamma}_{2}\right\}$ with $\boldsymbol{\Gamma}_{1} \in \mathbb{R}^{m \times m}, \boldsymbol{\Gamma}_{1}=\boldsymbol{\Gamma}_{1}^{\boldsymbol{\top}}>0, \gamma_{m+1}>0$, and $\boldsymbol{\Gamma}_{2} \in$ $\mathbb{R}^{n \times n}, \boldsymbol{\Gamma}_{2}=\boldsymbol{\Gamma}_{2}^{\boldsymbol{\top}}>0, \boldsymbol{\theta}_{0}$ is an initial estimate of $\boldsymbol{\theta}_{p}^{*}$, and $m(t)=\sqrt{1+\kappa \boldsymbol{\phi}^{\top}(t) \boldsymbol{\phi}(t)}$ with 
$\kappa>0$. The parameter projection term is $\boldsymbol{f}(t)=\left[\mathbf{0}_{1 \times m}, f_{m+1}(t), \mathbf{0}_{1 \times n}\right]^{\top}$ with $f_{m+1}(t)$ designed to ensure $\left|\theta_{p m+1}(t)\right|=\left|\hat{k}_{p}\right| \in\left[k_{p 0},+\infty\right)$, where $\theta_{p m+1}(t)$ is the $(m+1)$ th component of $\boldsymbol{\theta}_{p}(t)$, and $k_{p 0} \in\left(0,\left|k_{p}\right|\right]$ is a constant which is assumed to be known for implementing a parameter projection algorithm.

Controller Structure. The following control law

$$
u(t)=\boldsymbol{\theta}_{1}^{\boldsymbol{\top}}(t) \boldsymbol{\omega}_{1}(t)+\boldsymbol{\theta}_{2}^{\boldsymbol{\top}}(t) \boldsymbol{\omega}_{2}(t)+\theta_{20}(t) y(t)+\theta_{3}(t) r(t)
$$

is applied to the plant with the controller parameters $\boldsymbol{\theta}_{1}, \boldsymbol{\theta}_{2} \in \mathbb{R}^{n-1}, \theta_{20}, \theta_{3} \in \mathbb{R}$ calculated from the design equation

$$
\boldsymbol{\theta}_{1}^{\top} \boldsymbol{a}(s) \hat{P}(s, \hat{p})+\left(\boldsymbol{\theta}_{2}^{\boldsymbol{\top}} \boldsymbol{a}(s)+\theta_{20} \Lambda_{c}(s)\right) \hat{k}_{p} \hat{Z}(s, \hat{z})=\Lambda_{c}(s)\left(\hat{P}(s, \hat{p})-\hat{k}_{p} \theta_{3} \hat{Z}(s, \hat{z}) P_{m}(s)\right)
$$

where $\hat{P}(s, \hat{p})=s^{n}+\hat{p}_{n-1} s^{n-1}+\cdots+\hat{p}_{0}$, and $\hat{Z}(s, \hat{z})=s^{m}+\hat{z}_{m-1} s^{m-1}+\cdots+\hat{z}_{0}$ are constructed from $\boldsymbol{\theta}_{p}^{\top}(t)=\left[\widehat{k_{p} z_{0}}(t), \ldots, \widehat{k_{p} z_{m-1}}(t), \hat{k}_{p}(t),-\hat{p}_{0}(t), \ldots,-\hat{p}_{n-1}(t)\right]$, and $\hat{z}_{i}(t)=\frac{\widehat{k_{p}} z_{i}(t)}{\hat{k}_{p}(t)}, i=0,1, \ldots, m-1$, and $\Lambda_{c}(s)$ is a monic stable polynomial of degree $n-1$.

For $k=1$, the controller (3.5.5) with $\boldsymbol{\theta}_{i}(t)=\boldsymbol{\theta}_{i}, i=1,2,20,3$, adaptive law (3.5.4) and the design equation (3.5.6) for the plant (3.5.1) ensures that all signals in the closed-system are bounded and $\lim _{t \rightarrow \infty}\left(y(t)-y_{m}(t)\right)=0[15]$.

Gain Margin Analysis. The same closed-loop stability properties and asymptotic tracking performance hold for any constant $k>0$ within a specified range; that is, we have the gain margin result:

Proposition 3.5.1. A continuous-time SISO indirect MRAC system has gain margin $\left[\frac{k_{p 0}}{\left|k_{p}\right|},+\infty\right)$, where $k_{p 0}>0$ is the lower bound of $\left|k_{p}\right|$, used in the adaptive law (3.5.4).

Proof: When the constant gain $k$ is different from 1, the plant's high frequency gain becomes $k_{p} k$, and the parameter $\boldsymbol{\theta}_{p}(t)$ from the adaptive law (3.5.4) becomes the 
estimate of the new parameter vector

$$
\boldsymbol{\theta}_{p k}^{*}=\left[k_{p} k z_{0}, \ldots, k_{p} k z_{m-1}, k_{p} k,-p_{0}, \ldots,-p_{n-1}\right]^{\top}
$$

The controller (3.5.5) with the controller parameters computed from (3.5.6) still ensures desired closed-loop performance if $\left|k_{p} k\right| \geq k_{p 0}>0$, i.e., $k \geq \frac{k_{p 0}}{\left|k_{p}\right|}$, which is derived from the assumption based on which (3.5.4) is designed for $k=1$. Therefore, the continuous-time indirect MRAC design has gain margin $\left[\frac{k_{p 0}}{\left|k_{p}\right|},+\infty\right)$.

\subsubsection{Design for Discrete-Time SISO Systems}

Consider the discrete-time plant model (3.3.6), i.e.,

$$
y(t)=G(z)[u](t), \quad G(z)=k_{p} \frac{Z(z)}{P(z)}
$$

where $k_{p}$ is the plant high frequency gain, $y(t) \in \mathbb{R}$ is the plant output, $u(t) \in \mathbb{R}$ is the plant input, and

$$
Z(z)=z^{m}+z_{m-1} z^{m-1}+\cdots+z_{1} z+z_{0}, \quad P(z)=z^{n}+p_{n-1} z^{n-1}+\cdots+p_{1} z+p_{0}
$$

are polynomials in $z$ with $z_{i}, i=0,1, \ldots, m-1$, and $p_{j}, j=0,1, \ldots, n-1, n>m$, being unknown but constant parameters. It can be parameterized as $y(t)=\boldsymbol{\theta}_{p}^{* \top} \boldsymbol{\phi}(t)$, $t \in\{0,1, \ldots\}$ where $\boldsymbol{\theta}_{p}^{*}$ is as in (3.5.2), and the regressor vector is $\phi(t)=[u(t-n), u(t-n+1), \ldots, u(t-n+m), y(t-n), y(t-n+1), \ldots, y(t-1)]^{\top}$

Similar to the continues-time design, Assumption (A3.5-1) is needed, in addition to the standard MRAC assumptions (see Section 3.4.1). The reference model transfer function in (3.1.3) is taken to be $W_{m}(z)=z^{-n^{*}}$ such that $y_{m}\left(t+n^{*}\right)=r(t)$.

Error Model and Adaptive Law. Let $\boldsymbol{\theta}_{p}(t)$ be the estimate of $\boldsymbol{\theta}_{p}^{*}$, based on the estimation error model

$$
\epsilon(t)=\boldsymbol{\theta}_{p}^{\top}(t) \phi(t)-y(t)
$$


the following adaptive law is applied to update $\boldsymbol{\theta}_{p}(t)$ :

$$
\boldsymbol{\theta}_{p}(t+1)=\boldsymbol{\theta}_{p}(t)-\frac{\boldsymbol{\Gamma} \phi(t) \epsilon(t)}{m^{2}(t)}+\boldsymbol{f}(t), \boldsymbol{\theta}_{p}(0)=\boldsymbol{\theta}_{0}, t \in\{0,1, \ldots\}
$$

where the adaptation gain matrix $\boldsymbol{\Gamma}=\operatorname{diag}\left\{\boldsymbol{\Gamma}_{1}, \gamma_{m+1}, \boldsymbol{\Gamma}_{2}\right\}$ with $\boldsymbol{\Gamma}_{1} \in \mathbb{R}^{m \times m}, 0<$ $\boldsymbol{\Gamma}_{1}=\boldsymbol{\Gamma}_{1}^{\boldsymbol{\top}}<2 \boldsymbol{I}_{m}, 0<\gamma_{m+1}<2$, and $\boldsymbol{\Gamma}_{2} \in \mathbb{R}^{n \times n}, 0<\boldsymbol{\Gamma}_{2}=\boldsymbol{\Gamma}_{2}^{\boldsymbol{\top}}<2 \boldsymbol{I}_{n}, \boldsymbol{\theta}_{0}$ is an initial estimate of $\boldsymbol{\theta}_{p}^{*}$, and $m(t)=\sqrt{\kappa+\boldsymbol{\phi}^{\top}(t) \boldsymbol{\phi}(t)}$ with $\kappa>0$. The parameter projection term is $\boldsymbol{f}(t)=\left[\mathbf{0}_{1 \times m}, f_{m+1}(t), \mathbf{0}_{1 \times n}\right]^{\top}$ with $f_{m+1}(t)$ designed to ensure $\left|\theta_{p m+1}(t)\right|=$ $\left|\hat{k}_{p}\right| \in\left[k_{p 0},+\infty\right.$ ) (using the knowledge of $k_{p 0} \leq\left|k_{p}\right|$ ), where $\theta_{p m+1}(t)$ is the $(m+1)$ th component of $\boldsymbol{\theta}_{p}(t)$.

Controller Structure. The control law (3.4.2), i.e.,

$$
u(t)=\boldsymbol{\theta}_{1}^{\top}(t) \boldsymbol{\omega}_{1}(t)+\boldsymbol{\theta}_{2}^{\top}(t) \boldsymbol{\omega}_{2}(t)+\theta_{3}(t) y_{m}\left(t+n^{*}\right)
$$

is applied to the plant (3.5.8) with the controller parameters $\boldsymbol{\theta}_{i}(t)=\boldsymbol{\theta}_{i}, i=1,2,3$, computed from the design equation

$$
\boldsymbol{\theta}_{1}^{\top} \boldsymbol{a}_{\lambda}(z) \hat{P}(z, \hat{p})+\boldsymbol{\theta}_{2}^{\top} \boldsymbol{b}_{\lambda}(z) \hat{k}_{p} \hat{Z}(z, \hat{z})=\hat{P}(z, \hat{p})-\hat{k}_{p} \theta_{3} \hat{Z}(z, \hat{z}) z^{n^{*}}
$$

where $\hat{P}(z, \hat{p})$ and $\hat{Z}(z, \hat{z})$ are in the same form as in (3.5.6) with $s$ replaced by $z$.

For $k=1$, the controller (3.5.11) with the design equation (3.5.6) and the adaptive law (3.5.10) ensures closed-loop signal boundedness and $\lim _{t \rightarrow \infty}\left(y(t)-y_{m}(t)\right)=0$ [15].

Gain Margin Analysis. The same properties hold for any constant $k$ within a specified range; that is, we have the gain margin result:

Proposition 3.5.2. A discrete-time SISO indirect MRAC system has gain margin $\left[\frac{k_{p 0}}{\left|k_{p}\right|},+\infty\right)$, where $k_{p 0}>0$ is the lower bound of $\left|k_{p}\right|$, used in the adaptive law (3.5.10).

Proof: When the positive constant gain $k$ is not equal to 1 , the high frequency gain of the plant becomes $k_{p} k$, and $\boldsymbol{\theta}_{p}(t)$ becomes the estimate of the new parameter vector 
as in (3.5.7). The control law (3.5.11) still ensures desired closed-loop performance if $\left|k_{p} k\right| \geq k_{p 0}>0$, i.e., $k \geq \frac{k_{p 0}}{\left|k_{p}\right|}$, which is derived from the assumption based on which (3.5.10) is designed for $k=1$. Therefore, the discrete-time indirect MRAC design has gain margin $\left[\frac{k_{p 0}}{\left|k_{p}\right|},+\infty\right)$.

Remark 3.5.3. Comparing the conclusion with that of the discrete-time SISO direct $M R A C$ design, we can claim that if a larger system gain margin is desired, indirect MRAC scheme can be considered as an alternative.

Remark 3.5.4. Similar to Remark 3.4.2, some $k \in\left(0, \frac{k_{p 0}}{\left|k_{p}\right|}\right)$ may result in a stable system under some initial conditions, and the range of $k$ in Propositions 3.5 .1 and 3.5.2 are only sufficient conditions for closed-loop stability and asymptotic output tracking of the adaptive control systems.

\subsubsection{Design for A Class of Multivariable Systems}

Elliott and Wolovich's Algorithm. In [78], Elliott and Wolovich used the left coprime polynomial matrix decomposition of the system transfer matrix in developing indirect adaptive control strategies, because this parametrization can be estimated by input and output data. In this case, the system is expressed as

$$
\boldsymbol{y}(t)=\boldsymbol{G}(D)[\boldsymbol{u}](t), \quad \boldsymbol{G}(D)=\boldsymbol{P}_{l}^{-1}(D) \boldsymbol{Z}_{l}(D)
$$

where $\boldsymbol{Z}_{l}(D)$ and $\boldsymbol{P}_{l}(D)$ are left coprime polynomial matrices in $D$ with $\boldsymbol{P}_{l}(D)$ being row reduced.

Assume the observability indices of $\boldsymbol{G}(D)$, denoted as $\nu_{i}, i=1,2, \ldots, M$, are known, and let $\nu=\max _{1 \leq i \leq M} \nu_{i}$, the observability index of $\boldsymbol{G}(D)$. Without loss of generality, assume the row degrees of $\boldsymbol{P}_{l}^{\top}(D)$ are $\partial_{c i}\left(\boldsymbol{P}_{l}^{\top}(D)\right)=\nu_{i}$, and the matrix $\boldsymbol{P}_{\nu} \in \mathbb{R}^{M \times M}$, containing the coefficients of the $D^{\nu_{i}}$ term in each column of $\boldsymbol{P}_{l}(D)$, is 
unity lower triangular. By filtering the input-output equation of the system model, a parametrization linear in the unknown plant parameters contained in $\boldsymbol{Z}_{l}(D)$ and $\boldsymbol{P}_{l}(D)$ can be obtained, based on which standard adaptive estimation techniques can be used for estimation of the plant parameters.

The transfer matrix $\boldsymbol{W}_{m}(D)$ of the reference model is chosen to be $\boldsymbol{W}_{m}(D)=$ $\boldsymbol{\xi}_{m}^{-1}(D)$, and the controller structure is in the form of (3.3.14), i.e.,

$$
\boldsymbol{u}(t)=\boldsymbol{\Theta}_{1}^{\top} \boldsymbol{\omega}_{1}(t)+\boldsymbol{\Theta}_{2}^{\top} \boldsymbol{\omega}_{2}(t)+\boldsymbol{\Theta}_{20} \boldsymbol{y}(t)+\boldsymbol{\Theta}_{3} \boldsymbol{r}(t)
$$

with the parameters obtained from the design equation:

$$
\boldsymbol{I}_{M}-\boldsymbol{\Theta}_{1}^{\top} \boldsymbol{F}(D)-\left(\boldsymbol{\Theta}_{2}^{\top} \boldsymbol{F}(D)+\boldsymbol{\Theta}_{20}\right) \hat{\boldsymbol{G}}(D)=\boldsymbol{\Theta}_{3} \boldsymbol{\xi}_{m}(D) \hat{\boldsymbol{G}}(D)
$$

where $\hat{\boldsymbol{G}}(D)=\hat{\boldsymbol{P}}_{l}^{-1}(D) \hat{\boldsymbol{Z}}_{l}(D)$ with $\hat{\boldsymbol{P}}_{l}(D)$ and $\hat{\boldsymbol{Z}}_{l}(D)$ being the estimates of $\boldsymbol{P}_{l}(D)$ and $\boldsymbol{Z}_{l}(D)$, and $\boldsymbol{F}(D)$ is defined as in Section 3.1 together with $\boldsymbol{\omega}_{1}(t)$ and $\boldsymbol{\omega}_{2}(t)$.

To solve the plant-model matching equation (3.5.14) online, we need to assume that $\hat{\boldsymbol{G}}(D)$ at each time instant $t$ has the same interactor matrix $\boldsymbol{\xi}_{m}(D)$, and the corresponding estimated high frequency gain matrix $\hat{\boldsymbol{K}}_{p}$ is nonsingular. However, since it is a row-by-row estimation, an algorithm ensuring a nonsingular $\hat{\boldsymbol{K}}_{p}$ is not available. Thus here we consider a special case, which is an expansion from the SISO indirect MRAC algorithms (see Sections 3.4.1 and 3.4.2).

Design for A Class of Multivariable Systems. We make the assumptions that the highest order coefficient matrix of $\boldsymbol{P}_{l}(D)$ is the identity matrix $\boldsymbol{I}_{M}$, and that of $\boldsymbol{Z}_{l}(D)$ is lower triangular with nonzero diagonal elements; that is,

$$
\begin{aligned}
& \boldsymbol{P}_{l}(D)=\operatorname{diag}\left\{D^{\nu_{1}}, \ldots, D^{\nu_{M}}\right\} \boldsymbol{P}_{\nu}+\text { lower degree terms } \\
& \boldsymbol{Z}_{l}(D)=\operatorname{diag}\left\{D^{\nu_{1}-1}, \ldots, D^{\nu_{M}-1}\right\} \boldsymbol{Z}_{\nu-1}+\text { lower degree terms }
\end{aligned}
$$

where $\boldsymbol{P}_{\nu}=\boldsymbol{I}_{M}$, and $\boldsymbol{Z}_{\nu-1}$ is lower triangular with the diagonal elements $z_{d i} \neq 0$, $i=1, \ldots, M$. Therefore, the plant transfer matrix $\boldsymbol{G}(D)$ has an interactor matrix $\boldsymbol{\xi}_{m}(D)=D \cdot \boldsymbol{I}_{M}$, and the plant high frequency gain matrix is $\boldsymbol{K}_{p}=\boldsymbol{Z}_{\nu-1}$. 
Note that $\operatorname{det}\left(\boldsymbol{Z}_{\nu-1}\right)=\prod_{i=1}^{M} z_{d i}$. To ensure a nonsingular estimation of $\boldsymbol{K}_{p}=$ $\boldsymbol{Z}_{\nu-1}$, we need to make the following assumption for $z_{d i}, i=1,2, \ldots, M$ :

(A3.5-2) the sign of $z_{d i}, \operatorname{sign}\left[z_{d i}\right]$, is known, and so is the lower bound $z_{d i 0}$ of $\left|z_{d i}\right|$ such that $0<z_{d i 0} \leq\left|z_{d i}\right|$.

Plant model parametrization. To obtain a parametrization of the plant model (3.5.12) with parameter matrices in (3.5.15), we filter both sides of (3.5.12) with $\frac{1}{\Lambda_{e}(D)}$ for a chosen monic stable polynomial $\Lambda_{e}(D)$ of degree $\nu$. After ignoring exponentially decaying terms, we can obtain

$$
\begin{aligned}
\overline{\boldsymbol{y}}(t) & \triangleq \operatorname{diag}\left\{D^{\nu_{1}}, \ldots, D^{\nu_{M}}\right\} \frac{1}{\Lambda_{e}(D)}[\boldsymbol{y}](t) \\
& =\left(-\boldsymbol{P}_{l}(D)+\operatorname{diag}\left\{D^{\nu_{1}}, \ldots, D^{\nu_{M}}\right\}\right) \frac{1}{\Lambda_{e}(D)}[\boldsymbol{y}](t)+\boldsymbol{Z}_{l}(D) \frac{1}{\Lambda_{e}(D)}[\boldsymbol{u}](t) \\
& =\left[\boldsymbol{\theta}_{1}^{* \top} \boldsymbol{\zeta}_{1}(t), \ldots, \boldsymbol{\theta}_{M}^{* \top} \boldsymbol{\zeta}_{M}(t)\right]^{\top}
\end{aligned}
$$

where $\boldsymbol{\theta}_{i}^{*} \in \mathbb{R}^{M\left(2 \nu_{i}-1\right)+i}$ contains the plant parameters; that is, the constant coefficients from the polynomial matrices $\boldsymbol{P}_{l}(D)$ and $\boldsymbol{Z}_{l}(D)$, and $\boldsymbol{\zeta}_{i}(t) \in \mathbb{R}^{M\left(2 \nu_{i}-1\right)+i}$ are the filtered vector signals of $\boldsymbol{u}(t)$ and $\boldsymbol{y}(t)$. Letting $\boldsymbol{\theta}_{i}(t)$ be the estimate of $\boldsymbol{\theta}_{i}^{*}$, and introducing the estimation errors $\epsilon_{i}(t)=\boldsymbol{\theta}_{i}^{\top}(t) \boldsymbol{\zeta}_{i}(t)-\bar{y}_{i}(t)$ for $\overline{\boldsymbol{y}}(t)=\left[\bar{y}_{1}(t), \ldots, \bar{y}_{M}(t)\right]^{T}$, we have the error equations

$$
\epsilon_{i}(t)=\tilde{\boldsymbol{\theta}}_{i}^{\top}(t) \boldsymbol{\zeta}_{i}(t), \quad \tilde{\boldsymbol{\theta}}_{i}(t)=\boldsymbol{\theta}_{i}(t)-\boldsymbol{\theta}_{i}^{*}
$$

Error model and adaptive law. Based on the error equations (3.5.16), we choose the gradient adaptive laws with parameter projection to update $\boldsymbol{\theta}_{i}(t)$ :

$$
\left.\begin{array}{r}
\dot{\boldsymbol{\theta}}_{i}(t) \\
\boldsymbol{\theta}_{i}(t+1)-\boldsymbol{\theta}_{i}(t)
\end{array}\right\}=-\frac{\boldsymbol{\Gamma}_{i} \boldsymbol{\zeta}_{i}(t) \epsilon_{i}(t)}{m^{2}(t)}+\boldsymbol{f}_{i}(t), \quad i=1,2, \ldots, M
$$

where $m^{2}(t)=1+\sum_{i=1}^{M} \boldsymbol{\zeta}_{i}^{\top}(t) \boldsymbol{\zeta}_{i}(t)$, and the adaptive gain matrix $\boldsymbol{\Gamma}_{i}=\operatorname{diag}\left\{\boldsymbol{\Gamma}_{i 1}, \gamma_{i}, \boldsymbol{\Gamma}_{i 2}\right\}$ with $\boldsymbol{\Gamma}_{i 1} \in \mathbb{R}^{M \nu_{i} \times M \nu_{i}}, \gamma_{i} \in \mathbb{R}, \boldsymbol{\Gamma}_{i 2} \in \mathbb{R}^{\left(M\left(\nu_{i}-1\right)+i-1\right) \times\left(M\left(\nu_{i}-1\right)+i-1\right)}$. For continuoustime case, $\boldsymbol{\Gamma}_{i 1}=\boldsymbol{\Gamma}_{i 1}^{\top}>\mathbf{0}, \boldsymbol{\Gamma}_{i 2}=\boldsymbol{\Gamma}_{i 2}^{\top}>\mathbf{0}, \gamma_{i}>0$, while for discrete-time case, 
$\mathbf{0}<\boldsymbol{\Gamma}_{i 1}=\boldsymbol{\Gamma}_{i 1}^{\top}<2 \boldsymbol{I}_{M}, \mathbf{0}<\boldsymbol{\Gamma}_{i 2}=\boldsymbol{\Gamma}_{i 2}^{\top}<2 \boldsymbol{I}_{\nu}$, and $0<\gamma_{i}<2$. The parameter projection term $\boldsymbol{f}_{i}(t)$ has the form $\boldsymbol{f}_{i}(t)=\left[\mathbf{0}_{1 \times M \nu_{i}}, f_{i, M \nu_{i}+1}(t), \mathbf{0}_{1 \times M\left(\nu_{i}-1\right)+i-1}\right]^{\top}$ with $f_{i, M \nu_{i}+1}(t)$ designed to ensure the estimate of the $\left(M \nu_{i}+1\right)$ th component of $\boldsymbol{\theta}_{i}(t), \hat{z}_{d i}$, to be away from zero; that is, $\left|\boldsymbol{\theta}_{i, M \nu_{i}+1}(t)\right|=\left|\hat{z}_{d i}\right| \in\left[z_{d i 0},+\infty\right)$ (using the knowledge of $\left.z_{d i 0} \leq\left|z_{d i}\right|, i=1,2, \ldots, M\right)$.

With the adaptive laws (3.5.17), the design equation (3.5.14), the control law (3.3.14) applied to the system (3.5.12) can achieve closed-loop signal boundedness and asymptotic output tracking.

Remark 3.5.5. With some minor modifications to the system parametrization, the indirect $M R A C$ design presented above can be readily extended to the case when $\boldsymbol{Z}_{\nu-1}$ in (3.5.15) is upper triangular. A similar design follows as well if (3.5.15) is modified based on some knowledge of the highest row degrees of $\boldsymbol{Z}_{l}(D)$, if available. Further extensions to the cases when the parameter matrices $\boldsymbol{Z}_{\nu-1}$ and $\boldsymbol{P}_{\nu}$ are of a more general form requires an adaptive estimation scheme that can ensure a nonsingular estimation of $\boldsymbol{K}_{p}$, which is still an open issue to be addressed.

Gain Margin Analysis. The same desired closed-loop performance holds for the case when a positive definite gain matrix $\boldsymbol{K}>0$ as in (3.1.2) is present at the control input, as long as the gains $k_{i}$ are within some lower-bounded ranges; that is, we have the GM result:

Proposition 3.5.6. The closed-loop system, consisting of the system (3.5.12) and the controller (3.5.13) with the adaptive laws (3.5.17) and design equation (3.5.14), in either continuous or discrete time, has gain margins $\left[\frac{z_{d i 0}}{\left|z_{d i}\right|},+\infty\right)$ for $k_{i}$ of $\boldsymbol{K}=$ $\operatorname{diag}\left\{k_{1}, k_{2}, \ldots, k_{M}\right\}$, where $z_{d i 0}$, used in the adaptive law (3.5.17), are the known lower bounds $f\left|z_{d i}\right|$ such that $0<z_{d i 0} \leq\left|z_{d i}\right|, i=1,2, \ldots, M$. 
Proof: In the presence of $\boldsymbol{K}$, the controlled system is $\boldsymbol{y}(t)=\boldsymbol{G}(D) \boldsymbol{K}[\boldsymbol{u}](t)$, and its high frequency gain matrix is $\boldsymbol{K}_{p} \boldsymbol{K}=\boldsymbol{Z}_{\nu-1} \boldsymbol{K}$, which needs to satisfy the assumptions under which the adaptive laws (3.5.17) are designed for $\boldsymbol{K}=\boldsymbol{I}_{M}$, in order for closedloop stability and asymptotic tracking to be achieved. That is, for $\boldsymbol{K} \neq \boldsymbol{I}_{M}$, the diagonal entries of $\boldsymbol{Z}_{\nu-1} \boldsymbol{K}$ must be greater than the assumed lower bounds $z_{d i 0}$, i.e., $\left|z_{d i} k_{i}\right| \geq z_{d i 0}$, from which we can obtain $k_{i} \in\left[\frac{z_{d i 0}}{\left|z_{d i}\right|},+\infty\right), i=1,2, \ldots, M$. Therefore, we have the stated GM result.

Remark 3.5.7. When $M=1$, the MIMO system (3.5.12) reduces to the SISO system $P(D)[y](t)=k_{p} Z(D)[u](t)$. For indirect SISO MRAC design, we make the assumption that the lower bound $k_{p 0}$ of $\left|k_{p}\right|$ such that $0<k_{p 0} \leq\left|k_{p}\right|$ is known, and have derived the gain margin for $k$ to be $\left[\frac{k_{p 0}}{\left|k_{p}\right|},+\infty\right)$ (see Sections 3.4.1 and 3.4.2). We can see that the GM result in Proposition 3.5.6 is a direct extension from the SISO result.

Remark 3.5.8. This result is similar to that for the prediction model based adaptive (one-step-ahead) control scheme [77] (see Section 3.4.2), whose gain margin has an infinite upper bound but with a finite lower bound determined by the lower bound on $\left|k_{p}\right|$ (for the SISO case) used in parameter projection for ensuring a nonsingular control implementation.

Remark 3.5.9. For both continuous-time and discrete-time indirect MRAC design$s$, the gain margin is $\left[k_{i 0},+\infty\right)$, where $k_{i 0}>0$ can be made small by reducing the parameter lower bound used in parameter projection of the adaptive laws (for plant identification) for avoiding control singularity. 


\subsection{Gain Margins of Sampled-Data Adaptive Con- trol Systems}

Sampled-data systems are a special class of discrete-time systems in that their dynamics is obtained by discretizing continuous-time system dynamics with sample-and-hold devices operating on the inputs. It is thus of interest to study the effect of sample time on the gain margin.

\subsubsection{MRAC of Sampled-Data Systems}

Consider a strictly proper continuous-time system

$$
\begin{aligned}
\dot{\boldsymbol{x}}(t) & =\boldsymbol{A} \boldsymbol{x}(t)+\boldsymbol{B} \boldsymbol{u}(t) \\
\boldsymbol{y}(t) & =\boldsymbol{C} \boldsymbol{x}(t)
\end{aligned}
$$

with unknown parameter matrices $\boldsymbol{A} \in \mathbb{R}^{n \times n}, \boldsymbol{B} \in \mathbb{R}^{n \times M}$ and $\boldsymbol{C} \in \mathbb{R}^{M \times n}$, and the system input and output vectors $\boldsymbol{u}(t), \boldsymbol{y}(t) \in \mathbb{R}^{M}$. Its transfer matrix representation is $\boldsymbol{G}(s)=\boldsymbol{C}(s \boldsymbol{I}-\boldsymbol{A})^{-1} \boldsymbol{B}$. The solution to the state equation in (3.6.1) is

$$
\boldsymbol{x}(t)=e^{\boldsymbol{A}\left(t-t_{0}\right)} \boldsymbol{x}\left(t_{0}\right)+\int_{t_{0}}^{t} e^{\boldsymbol{A}(t-\tau)} \boldsymbol{B} \boldsymbol{u}(\tau) d \tau, \quad t>t_{0} \geq 0
$$

To find a discrete-time model for this system, we consider the state transition within one sample period $T$. When the plant inputs are preceded by samplers and zero-order holds (ZOH), we have $\boldsymbol{u}(\tau)=\boldsymbol{u}(k T), k T \leq \tau<(k+1) T$, where $k$ is any nonnegative integer. Setting $t=(k+1) T$ and $t_{0}=k T$ in (3.6.2) leads to the sampled-data system in the state space form:

$$
\begin{aligned}
\boldsymbol{x}((k+1) T) & =\boldsymbol{A}_{d} \boldsymbol{x}(k T)+\boldsymbol{B}_{d} \boldsymbol{u}(k T) \\
\boldsymbol{y}(k T) & =\boldsymbol{C}_{d} \boldsymbol{x}(k T), \quad k=0,1,2, \ldots
\end{aligned}
$$

with $\boldsymbol{A}_{d}=e^{\boldsymbol{A} T}, \boldsymbol{B}_{d}=\int_{0}^{T} e^{\boldsymbol{A} \tau} d \tau \boldsymbol{B}$, and $\boldsymbol{C}_{d}=\boldsymbol{C}$; furthermore, the transfer matrix of the system is

$$
\boldsymbol{G}(z)=\boldsymbol{C}_{d}\left(z \boldsymbol{I}-\boldsymbol{A}_{d}\right)^{-1} \boldsymbol{B}_{d}
$$


To apply MRAC schemes to the sampled-data system (3.6.3), the respective design conditions in discrete time must be satisfied after sampling of the system (3.6.1), then direct (Section 3.4) or indirect (Section 3.5) designs can be applied. Note that for direct MRAC, a minimum-phase condition is required; however, it is well known that some minimum-phase continuous-time systems may become nonminimum-phase after sampling, especially when $T$ is small [79]. Moreover, the zero structure at infinity, characterized by the modified interactor matrix $\boldsymbol{\xi}_{m}(z)$, for choosing the reference model system, may not be fixed; that is, it depends on the sample time $T$ and may change for different $T$.

\subsubsection{Gain Margin Analysis}

For each value of $T$, the GM of the sampled-data adaptive control system can be computed based on Propositions 3.4.5 and 3.5.6. Here we explore the effect of sample time $T$ on the GM.

Direct MRAC. Recall that the GM for discrete-time direct MRAC systems is finite with upper bounds: $k_{i} \in\left(0, k_{i}^{0}\right]$, where $k_{i}^{0}$ depend on the system high frequency gain matrix $\boldsymbol{K}_{p}$, as well as some upper bounds of $\boldsymbol{K}_{p}$ that are used for parameter adaptation. In particular, the GM result reduces to $k \in\left(0, \frac{k_{p}^{0}}{\left|k_{p}\right|}\right]$ in the SISO case, where $k_{p}^{0}$ is the known upper bound of $\left|k_{p}\right|$ such that $0<\left|k_{p}\right| \leq k_{p}^{0}$. For sampled-data systems, $\boldsymbol{K}_{p}$ depends on $T$, and to study its effect on the GM, we focus on such systems with the minimum-phase property invariant under sampling with sample time T. Sufficient stability conditions exist for zeros of SISO [80] as well as MIMO sampled-data systems [81].

For simplicity we may assume a uniform modified interactor matrix, i.e., $\boldsymbol{\xi}_{m}(z)=$ $z^{n^{*}} \boldsymbol{I}_{M}$, where $n^{*}$ is the uniform vector relative degree of $\boldsymbol{G}(z)$ in (3.6.4). The high 
frequency gain matrix $\boldsymbol{K}_{p}$ can then be computed as

$$
\boldsymbol{K}_{p}=\lim _{z \rightarrow \infty} \boldsymbol{\xi}_{m}(z) \boldsymbol{G}(z)=\boldsymbol{C}_{d} \boldsymbol{A}_{d}^{n^{*}-1} \boldsymbol{B}_{d}
$$

Since $\boldsymbol{B}_{d}=\int_{0}^{T} e^{\boldsymbol{A} \tau} d \tau \boldsymbol{B}$, it is obvious that $\left\|\boldsymbol{B}_{d}\right\|$ decreases when $T$ is reduced. When $T$ becomes small, from (3.6.5) we can see that $\left\|\boldsymbol{K}_{p}\right\|$ decreases, which leads to an enlarging GM. In the limiting case, we have $\lim _{T \rightarrow 0}\left\|\boldsymbol{K}_{p}\right\|=0$, resulting in the GM being infinity, which can be readily verified by (3.4.14) for the case when $\boldsymbol{K}_{p}$ is lower triangular, and by (3.4.17) and (3.4.18) for the $M=2$ case. Note, however, the GM may not increase monotonously with decreasing $T$.

To illustrate the effect of $T$ on the GM, we consider a second-order SISO continuoustime system in (3.6.1) with transfer function $G(s)=(3 s+1) /[(s+4)(s+5)]$. It can be verified by (3.6.4) that after sampling, we have

$$
\begin{aligned}
G(z) & =\frac{k_{p}\left(z+z_{0}\right)}{\left(z-e^{-4 T}\right)\left(z-e^{-5 T}\right)} \\
k_{p} & =-\frac{8}{3}\left(1-e^{-3 T}\right)+\frac{11}{4}\left(1-e^{-4 T}\right)>0 \\
z_{0} & =\frac{1}{k_{p}}\left(-\frac{8}{3} e^{-4 T}+\frac{11}{4} e^{-3 T}-\frac{1}{12} e^{-7 T}\right)<1
\end{aligned}
$$

for all $T>0$; that is, the sampled-data system is minimum-phase with relative degree $n^{*}=1$. Thus $k_{p}$ is the high frequency gain with $\operatorname{sign}\left[k_{p}\right]>0$, and a modified interactor is $\xi_{m}(z)=z$. For direct MRAC design, the upper bound of $\left|k_{p}\right|$ is assumed to be known. If we pick $k_{p}^{0}=0.5$ in the design, the GM of the closed-loop system is $\left(0, \frac{0.5}{\left|k_{p}\right|}\right]$. The magnitude of $k_{p}$ and the upper bound of the GM are plotted in Fig. 3.2. Clearly, $\lim _{T \rightarrow 0}\left|k_{p}\right|=0$, and the upper bound on GM tends to $\infty$, which is exactly the GM for the corresponding continuous-time MRAC system. Note that the GM does not increase monotonously with decreasing $T$.

Indirect MRAC. Unlike direct MRAC, the GM for discrete-time indirect MRAC systems is infinite for upper bound but with finite lower bounds, that is, 

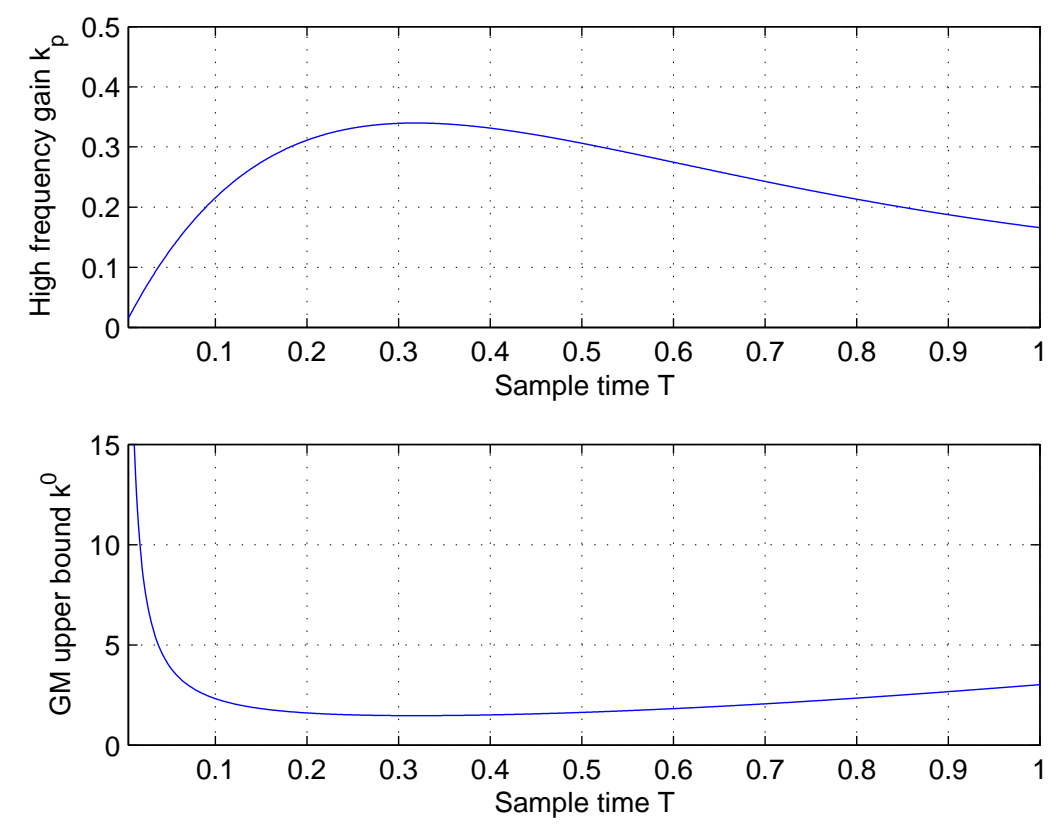

Figure 3.2: High frequency gain $k_{p}$ and GM upper bound $k^{0}$ versus $T$.

$k_{i} \in\left[k_{i 0},+\infty\right)$, where $k_{i 0}>0$ depend on the system high frequency gain matrix $\boldsymbol{K}_{p}$, as well as some lower bounds of $\boldsymbol{K}_{p}$ that are used for plant identification. In particular, the GM result reduces to $k \in\left[\frac{k_{p 0}}{\left|k_{p}\right|},+\infty\right)$ in the SISO case, where $k_{p 0}$ is the known lower bound of $\left|k_{p}\right|$ such that $0<k_{p 0} \leq\left|k_{p}\right|$. Since an algorithm for ensuring nonsingular estimation of $\boldsymbol{K}_{p}$ of a general plant in (3.5.12) is complicated, to show the effect of $T$ on the GM, we consider the class of sampled-data systems that can be represented by (3.5.15). Furthermore, we need to assume that the design conditions such as knowledge of the observability indices and the lower bound information stated in Assumption (A3.5-2) are invariant under sampling. For a system whose modified interactor matrix is $\boldsymbol{\xi}_{m}(z)=z \boldsymbol{I}_{M}$, the high frequency gain matrix is

$$
\boldsymbol{K}_{p}=\lim _{z \rightarrow \infty} \boldsymbol{\xi}_{m}(z) \boldsymbol{G}(z)=\boldsymbol{C}_{d} \boldsymbol{B}_{d}=\boldsymbol{Z}_{\nu-1}
$$

where $\boldsymbol{Z}_{\nu-1}$ is the highest order coefficient matrix of the numerator polynomial matrix 
in a left coprime decomposition of $\boldsymbol{G}(z)$. From (3.6.7) we can see that a decreasing $T$ causes $\left\|\boldsymbol{K}_{p}\right\|$ to decrease, and when $T$ becomes sufficiently small, the magnitude of the diagonal elements of $\boldsymbol{K}_{p},\left|z_{d i}\right|, i=1,2, \ldots, M$, decrease with $T$. This leads to a shrinking GM (its lower bounds are increasing), which vanishes in the limiting case as $T \rightarrow 0$.

The effect of $T$ on the GM can also be illustrated with the sampled-data system (3.6.6) if indirect MRAC is applied. The GM of this system is $\left[\frac{k_{p 0}}{\left|k_{p}\right|}, \infty\right)$. Figure 3.2 shows that $k_{p}$ decreases when $T$ becomes small, and the GM shrinks and vanishes as $T \rightarrow 0$.

\subsection{Simulation Study}

In this section we present an $M=2$ example to illustrate the gain margins of discretetime direct MRAC design.

We consider a controlled plant (3.1.1) with $\boldsymbol{u}(t), \boldsymbol{y}(t) \in \mathbb{R}^{2}$, and the transfer matrix

$$
\boldsymbol{G}(z)=\left[\begin{array}{cc}
\frac{1}{z-0.5} & \frac{0.6}{z+0.3} \\
\frac{1}{z+0.5} & \frac{1}{z+0.7}
\end{array}\right]
$$

which can be expressed as $\boldsymbol{G}(z)=\boldsymbol{Z}(z) \boldsymbol{P}^{-1}(z)$, where

$\boldsymbol{Z}(z)=\left[\begin{array}{cc}z+0.5 & 0.6(z+0.7) \\ z-0.5 & z+0.3\end{array}\right], \boldsymbol{P}(z)=\operatorname{diag}\{(z+0.5)(z-0.5),(z+0.3)(z+0.7)\}$

with observability index $\nu=2$. It can be verified that $\boldsymbol{G}(z)$ is minimum-phase, and $\boldsymbol{Z}(z)$ and $\boldsymbol{P}(z)$ are right coprime with $\boldsymbol{P}(z)$ column proper.

The modified interactor matrix and the associated high frequency gain matrix are

$$
\boldsymbol{\xi}_{m}(z)=\left[\begin{array}{cc}
z & 0 \\
0 & z
\end{array}\right], \quad \boldsymbol{K}_{p}=\left[\begin{array}{cc}
1 & 0.6 \\
1 & 1
\end{array}\right]
$$

from which we have $d_{1}^{*}=1, d_{2}^{*}=0.4, \operatorname{sign}\left[d_{1}^{*}\right]>0$ and $\operatorname{sign}\left[d_{2}^{*}\right]>0$. With $d_{1}^{0}=1.2$ and $d_{2}^{0}=1.0$, the standard MRAC Assumptions (A3.1-1)-(A3.1-4D) are all satisfied. 
Moreover, the unique LDU decomposition of $\boldsymbol{K}_{p}$ is

$$
\boldsymbol{L}=\left[\begin{array}{ll}
1 & 0 \\
1 & 1
\end{array}\right], \quad \boldsymbol{D}^{*}=\left[\begin{array}{cc}
1 & 0 \\
0 & 0.4
\end{array}\right], \quad \boldsymbol{U}=\left[\begin{array}{cc}
1 & 0.6 \\
0 & 1
\end{array}\right]
$$

The reference model is chosen to be (3.1.3) with the transfer matrix $\boldsymbol{W}_{m}(z)=$ $\boldsymbol{\xi}_{m}^{-1}(z)$ and the reference input $\boldsymbol{r}(t)=10[\cos (0.15 t), \sin (0.1 t)+2 \sin (0,2 t)]^{\top}$.

The controller structure is (3.3.14). With the specifications of $\Lambda(z)=z, \boldsymbol{\xi}_{m}(z)$, $\boldsymbol{P}(z)$, and $\boldsymbol{Z}(z)$ as above, its nominal parameter matrices $\boldsymbol{\Theta}_{1}^{*}, \boldsymbol{\Theta}_{2}^{*}, \boldsymbol{\Theta}_{20}^{*}, \boldsymbol{\Theta}_{3}^{*}=\boldsymbol{K}_{p}^{-1}$ can be computed from (3.3.2).

The adaptive laws are (3.4.7) with the adaptation gain (matrix) chosen as $\Gamma_{\theta_{2}}=1$ and $\boldsymbol{\Gamma}=\boldsymbol{I}_{2}$. From (3.4.15) and (3.4.16), $\gamma_{1}$ and $\gamma_{2}$ must be chosen such that $\gamma_{1} \in$ $(0,5 / 3)$ and $\gamma_{2} \in(0,0.9745)$. In the simulation, we pick $\gamma_{1}=1$ and $\gamma_{2}=0.8$, and thus we can obtain $\boldsymbol{L}_{s}=\boldsymbol{L} \boldsymbol{D}_{s}\left(\boldsymbol{U}^{\top}\right)^{-1} \boldsymbol{D}_{s}^{-1}, \boldsymbol{S}=\boldsymbol{U}^{\top} \boldsymbol{D}_{s}^{-1} \boldsymbol{D}^{*} \boldsymbol{U}$, and $\boldsymbol{\Psi}^{*}=\boldsymbol{D}_{s} \boldsymbol{S}$. Besides, $\theta_{2}^{*}$ can obtained from $\boldsymbol{\Theta}_{0}^{*}=\boldsymbol{L}_{s}^{-1}-\boldsymbol{I}_{2}$. The initial parameter estimates $\theta_{2}(0), \boldsymbol{\Theta}(0)$, and $\boldsymbol{\Psi}(0)$ are chosen as

$$
\theta_{2}(0)=0.95 \theta_{2}^{*}, \quad \Theta(0)=0.95 \Theta^{*}, \quad \Psi(0)=0.8 \Psi^{*}
$$

Note that the nominal parameters $\theta_{2}^{*}, \boldsymbol{\Theta}^{*}$, and $\boldsymbol{\Psi}^{*}$ are obtained for $\boldsymbol{K}=\boldsymbol{I}_{M}$ and depend on the design parameters in $\boldsymbol{D}_{s}$. For the gain matrix variations $\boldsymbol{K} \neq \boldsymbol{I}_{M}$, the transfer matrices of the plant and the reference model do not match each other with these parameters.

From the gain margin results in (3.4.17) and (3.4.18), we can obtain $k_{1} \in(0,1.2]$; the GM range of $k_{2}$ depends on $k_{1}$. With $k_{1}=0.9$, we have $k_{2} \in(0,1.4427]$. Simulations are performed for three cases, depending on different scenarios of gain matrix variation: (1) $\boldsymbol{K}=\operatorname{diag}\left\{k_{1}, k_{2}\right\}=\operatorname{diag}\{0.9,1.3\} ;$ (2) $\boldsymbol{K}=\operatorname{diag}\{0.9,1.8\} ;$ and (3) $\boldsymbol{K}=\operatorname{diag}\{0.9,1.5\}$. The output tracking error is plotted in Figures 3.3-3.5, respectively. 

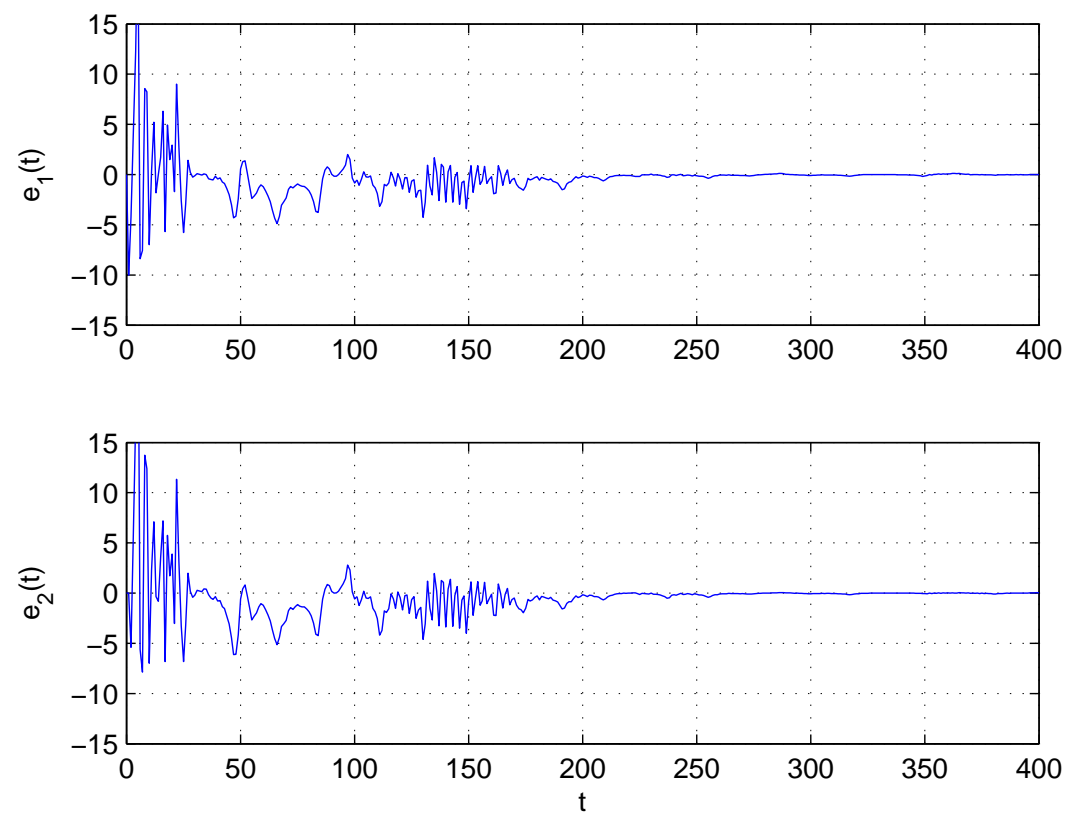

Figure 3.3: Output tracking error $\boldsymbol{e}(t)=\left[e_{1}(t), e_{2}(t)\right]^{\top}$ for $k_{1}=0.9 \in(0,1.2]$ and $k_{2}=1.3 \in(0,1.4427]$.
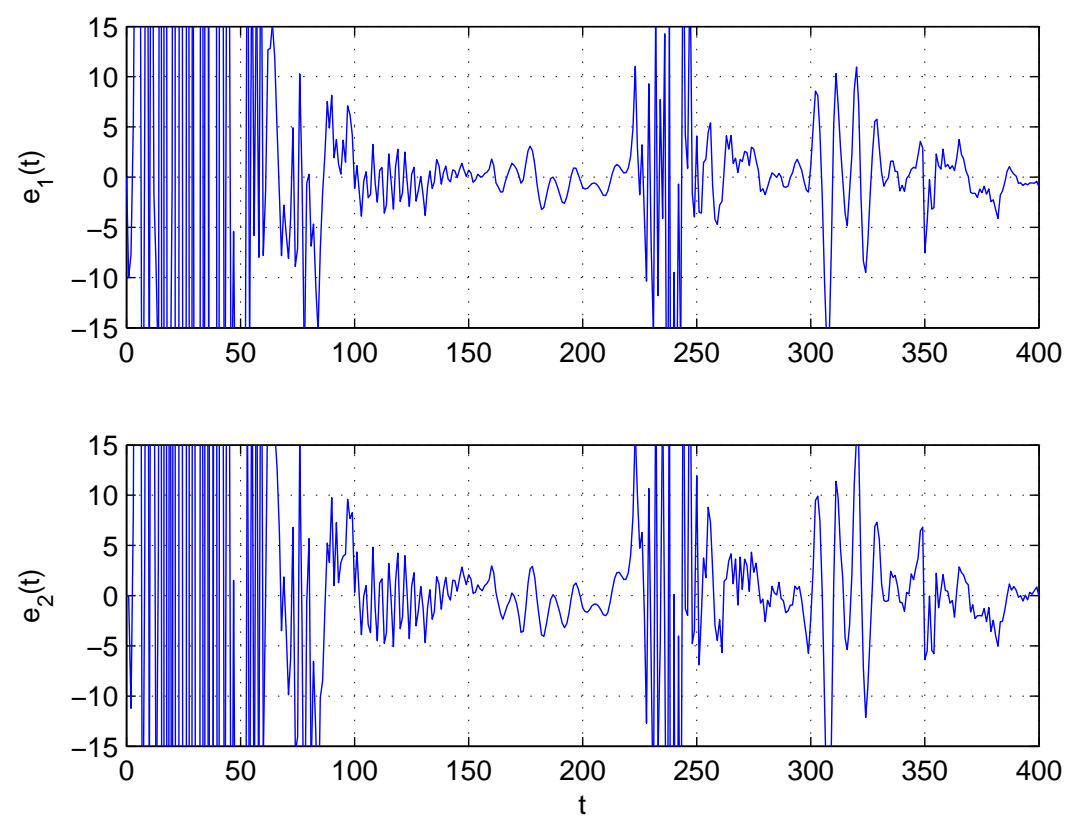

Figure 3.4: Output tracking error $\boldsymbol{e}(t)=\left[e_{1}(t), e_{2}(t)\right]^{\top}$ for $k_{1}=0.9 \in(0,1.2]$ and $k_{2}=1.8 \notin(0,1.4427]$. 

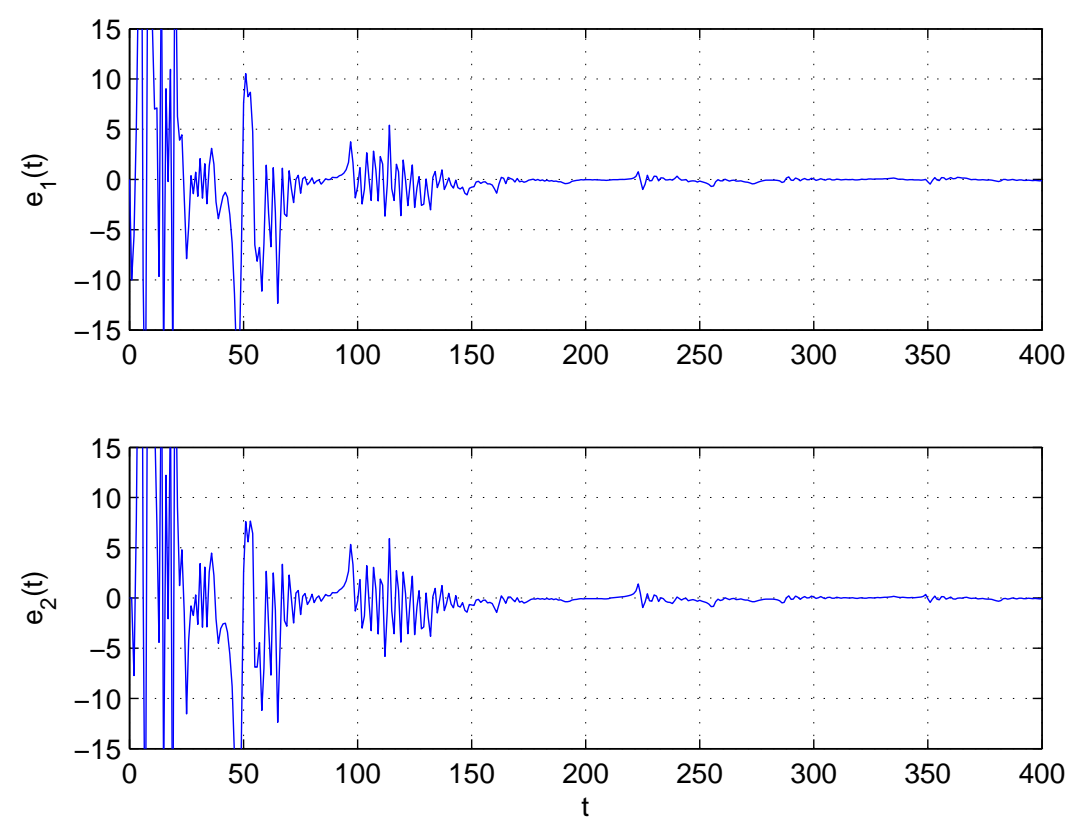

Figure 3.5: Output tracking error $\boldsymbol{e}(t)=\left[e_{1}(t), e_{2}(t)\right]^{\top}$ for $k_{1}=0.9 \in(0,1.2]$ and $k_{2}=1.5 \notin(0,1.4427]$.

From the simulation results, we can see that closed-loop signal boundedness and asymptotic tracking performance are achieved for gain matrix variation $\boldsymbol{K}$ inside the GM under any initial conditions (Fig. 3.3). For $\boldsymbol{K}$ outside the GM, the closed-loop system becomes unstable (Fig. 3.4), but if $\boldsymbol{K}$ is close to the GM upper bounds, the system can be stable under some initial conditions (Fig. 3.5). This example illustrates the main features of the gain margin of adaptive control systems: it is a sufficient range for control gain variation in which stability and asymptotic tracking performance can be maintained by the adaptive control design; when all initial conditions are considered, it is necessary as well.

In this chapter, the gain margin problem has been studied for several model reference adaptive control (MRAC) systems: those with direct or indirect, continuous-time or discrete-time designs. The obtained analytical gain margin results are also applica- 
ble to other adaptive control systems such as adaptive state feedback control systems and adaptive nonlinear control systems. For a direct continuous-time MRAC design, the gain margin is $(0,+\infty)$, while for a direct discrete-time design, the gain margin is finite with an upper bound that can be made large by reducing the adaptation gain. For indirect MRAC designs, in either continuous time or discrete time, the gain margin is infinity with a lower bound that can be made small by reducing the parameter lower bound used in parameter projection of adaptive laws (for plant identification) for avoiding control singularity. In other words, the gain margins of MRAC systems can be readily enlarged by choosing proper design parameters, while ensuring both signal boundedness and asymptotic tracking. The effect of sample time $T$ on the gain margin of sampled-data adaptive control systems is studied. It is shown that as $T \rightarrow 0$, for direct MRAC designs, the gain margin increases and coincides with the continuous-time result, while for indirect MRAC designs, the gain margin shrinks and vanishes. The gain margin results indicate that the use of a MRAC scheme has a significant advantage over a non-adaptive control scheme whose gain margin for signal boundedness may be limited and for asymptotic tracking may shrink to a fixed point (that is, any $\boldsymbol{K} \neq \boldsymbol{I}_{M}$ leads to a non-zero tracking error). On the other hand, it is interesting to compare the GM of adaptive control systems (especially those with a bounded GM range) with some non-adaptive control systems with well-developed control designs such as LQR, for the study of robustness of system stability (signal boundedness) with respect to control gain variations. 


\section{Chapter 4}

\section{Performance Robustness of MRAC with respect to Reduced Actuator Effectiveness}

In this chapter, the problem of performance robustness of model reference adaptive control (MARC) schemes with respect to reduced actuator effectiveness (loss-ofeffectiveness) is studied. Reduced actuator effectiveness is modeled by an uncertain gain matrix at the control inputs. MRAC systems are analyzed to determine the robustness of the desired closed-loop performance of stability and asymptotic tracking with respect to such reductions.

This chapter is organized as follows. In Section 4.1, the performance robustness problem is stated. We will show in Section 4.2 that the closed-loop performance of direct continuous-time multivariable MRAC designs is robust to arbitrary reduction in actuator effectiveness, while as proved in Section 4.3, that of their discrete-time counterparts is design-based. Indirect MRAC schemes are considered in Section 4.4. The main features of the performance robustness properties of the LDS based MRAC design are illustrated by simulation results in Section 4.5. 


\subsection{Performance Robustness Issue for MRAC with respect to Reduced Actuator Effectiveness}

Consider the $M$-input $M$-output linear time-invariant plant with transfer matrix representation:

$$
\boldsymbol{y}(t)=\boldsymbol{G}(D)\left[\boldsymbol{u}_{p}\right](t)
$$

where $\boldsymbol{u}_{p}(t), \boldsymbol{y}(t) \in \mathbb{R}^{M}$ are the plant input and output vectors, $\boldsymbol{G}(D)=\boldsymbol{Z}(D) \boldsymbol{P}^{-1}(D)$ is strictly proper and full rank, and $\boldsymbol{Z}(D), \boldsymbol{P}(D) \in \mathbb{R}^{M \times M}$ are right coprime polynomial matrices with $\boldsymbol{P}(D)$ being column proper. As in Chapter 3, the symbol $D$ is used, in the continuous-time case, as the time-differentiation operator: $D[x](t)=\dot{x}(t)$, $t \in[0,+\infty)$; or in the discrete-time case, as the time-advance operator: $D[x](t)=$ $x(t+1), t \in\{0,1,2,3, \ldots\}$.

Denoting $\boldsymbol{u}(t) \in \mathbb{R}^{M}$ as the control signal generated from an adaptive controller, and $\boldsymbol{K} \in \mathbb{R}^{M \times M}$ as the actuator effectiveness matrix that assumes the following form

$$
\boldsymbol{K}=\operatorname{diag}\left\{k_{1}, \ldots, k_{M}\right\}, 0<k_{i} \leq 1, \quad i=1,2, \ldots, M
$$

we have

$$
\boldsymbol{u}_{p}(t)=\boldsymbol{K} \boldsymbol{u}(t)
$$

In the absence of reduction in actuator effectiveness, $\boldsymbol{K}$ is an identity matrix $\boldsymbol{I}_{M} \in$ $\mathbb{R}^{M \times M}$, and $\boldsymbol{u}_{p}(t)=\boldsymbol{u}(t)$.

Figure 4.1 depicts the scenario we are considering in this chapter. The adaptive controller, denoted by $\boldsymbol{C}_{1}(D)$ and $\boldsymbol{C}_{2}(D)$, has been designed for $\boldsymbol{K}=\boldsymbol{I}_{M}$, and ensures closed-loop stability (signal boundedness) and asymptotic output tracking performance; that is, $\boldsymbol{y}(t)$ asymptotically tracks a desired trajectory $\boldsymbol{y}_{m}(t)$ generated from a reference model system

$$
\boldsymbol{y}_{m}(t)=\boldsymbol{W}_{m}(D)[\boldsymbol{r}](t)
$$




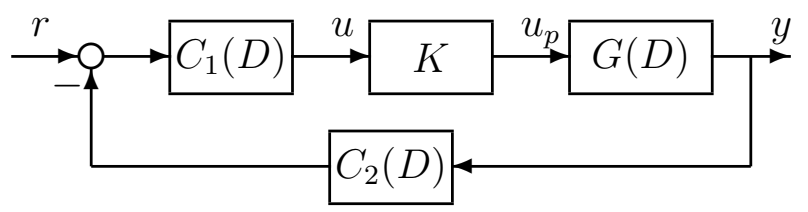

Figure 4.1: Adaptive control system with reduction in actuator effectiveness.

where $\boldsymbol{W}_{m}(D) \in \mathbb{R}^{M \times M}$ is a rational transfer matrix and $\boldsymbol{r}(t) \in \mathbb{R}^{M}$ is a bounded, piecewise continuous reference input signal. Our objective is to study whether or not the designed adaptive controller still ensures the desired closed-loop performance in the presence of reduction in actuator effectiveness, i.e., when $\mathbf{0}<\boldsymbol{K}<\boldsymbol{I}_{M}$.

The high frequency gain matrix is defined as $\boldsymbol{K}_{p}=\lim _{D \rightarrow \infty} \boldsymbol{\xi}_{m}(D) \boldsymbol{G}(D)$ with $\boldsymbol{\xi}_{m}(D)$ being the modified interactor matrix of $\boldsymbol{G}(D)$. For MRAC designs based on decompositions of $\boldsymbol{K}_{p}$, the reference model transfer matrix $\boldsymbol{W}_{m}(D)$ in (4.1.4) is chosen to be $\boldsymbol{W}_{m}(D)=\boldsymbol{\xi}_{m}^{-1}(D)$.

MRAC Assumptions. For MRAC designs, the standard assumptions are (A3.11)-(A3.1-4) for continuous-time designs, with an additional Assumption (A3.1-4D) for discrete-time designs (see Section 3.1).

Under these design conditions, we have presented in Chapter 3 direct and indirect MRAC schemes applied to both single-input, single-output (SISO) and multiinput, multi-output (MIMO) plants, in continuous time, as well as discrete time. The desirable closed-loop stability properties and asymptotic tracking performance are achieved for $\boldsymbol{K}=\boldsymbol{I}_{M}$.

For multivariable MRAC an important design condition is given for $\boldsymbol{K}_{p}$ in Assumption (A3.1-4). In the presence of reduction in actuator effectiveness, the high frequency gain matrix becomes $\boldsymbol{K}_{p} \boldsymbol{K}$ with $\boldsymbol{K}$ in (4.1.2). We note that Lemma 3.2.1 
(Section 3.1) is crucial for the performance robustness analysis of matrix decomposition based multivariable MRAC designs, as it relates the LDU decomposition of $\boldsymbol{K}_{p}$ with that of $\boldsymbol{K}_{p} \boldsymbol{K}$. From this lemma, we can conclude that the leading principle minors of $\boldsymbol{K}_{p} \boldsymbol{K}$ are also nonzero, and their sign information is the same as that of $\boldsymbol{K}_{p}$. In other words, the design conditions stated in Assumption (A3.1-4) are invariant in the presence of reduction in actuator effectiveness. Furthermore, Lemma 3.2.1 is crucial for obtaining the performance robustness conditions for direct discrete-time MRAC designs.

The GM results can be directly applied for solutions of the performance robustness problem with respect to reduced actuator effectiveness. As shown in the following sections, by comparing the GM results with the range of actuator effectiveness matrix defined in (4.1.2), we can determine whether or not the performance of an MRAC design is robust to and to what extent it can handle the reduction in actuator effectiveness.

\subsection{Continuous-Time MIMO Direct MRAC}

There are some well-known direct multivariable MRAC designs based on decompositions of $\boldsymbol{K}_{p}[15,73]$ (the LDS decomposition based design is presented in Section 3.3). To study their performance robustness with respect to reduced actuator effectiveness, the actuator effectiveness matrix $\boldsymbol{K}$, together with the plant (4.1.1) is treated as the new controlled plant.

The gain margins of continuous-time MRAC designs are infinity; that is, the closed-loop performance remains for any control gain at the control inputs, thus we have:

Proposition 4.2.1. The performance of direct continuous-time multivariable MRAC 
designs based on high frequency gain matrix decompositions is robust with respect to arbitrary reduction in actuator effectiveness.

Proof: From (4.1.1) and (4.1.3), we have

$$
\boldsymbol{y}(t)=\boldsymbol{G}(s) \boldsymbol{K}[\boldsymbol{u}](t)
$$

as the new controlled plant, and its high frequency gain matrix is $\boldsymbol{K}_{p} \boldsymbol{K}$. From (2.2.10) and (3.2.1), we can see that the presence of reduction in actuator effectiveness, i.e., $\mathbf{0}<\boldsymbol{K}<\boldsymbol{I}_{M}$ does not violate the assumptions of nonzero leading principle minors of the high frequency gain matrix under (A3.1-4), and their sign information is the same as that of $\boldsymbol{K}_{p}$. Therefore, the adaptive law for $\boldsymbol{K}=\boldsymbol{I}_{M}$ can still be used for $\mathbf{0}<\boldsymbol{K}<\boldsymbol{I}_{M}$ to achieve closed-loop performance of signal boundedness and asymptotic tracking. This is also true for direct continuous-time SISO MRAC designs.

The gain matrix decomposition based continuous-time MRAC designs can adaptively compensate for arbitrary reduction in actuator effectiveness to ensure desired closed-loop performance. However, for discrete-time designs, although the performance of SISO MRAC systems is robust with respect to reduction in actuator effectiveness, the performance robustness of multivariable MRAC systems are designbased, which is shown in the next section.

\subsection{Discrete-Time MIMO Direct MRAC}

We will first analyze the performance robustness properties of the MRAC design based on the LDS decomposition of $\boldsymbol{K}_{p}$ in this section. As a comparison, the LDU based design is analyzed to show that the performance robustness of the discrete-time MRAC systems under reduction in actuator effectiveness is design-based. 


\subsubsection{Performance Robustness of the LDS Based Design}

Among the gain matrix decomposition based MRAC designs, the LDS based design assumes a simple adaptive controller structure, and its error model parametrization and the auxiliary signal structures are simple for implementation, as shown in Section 3.4.2.

Similar to the analysis in Section 4.2 for continuous-time designs, the presence of reduction in actuator effectiveness does not violate the assumption of nonzero leading principle minors of the high frequency gain matrix, and their sign information is invariant with $\mathbf{0}<\boldsymbol{K}<\boldsymbol{I}_{M}$. However, for the aforementioned LDS based design to ensure the closed-loop signal boundedness and asymptotic tracking performance, the new condition for $\mathbf{0}<\boldsymbol{K}<\boldsymbol{I}_{M}$, similar to (3.4.8) for $\boldsymbol{K}=\boldsymbol{I}_{M}$, is

$$
\mathbf{0}<\boldsymbol{D}_{s} \overline{\boldsymbol{U}}^{\top} \boldsymbol{D}_{s}^{-1} \overline{\boldsymbol{D}}^{*} \overline{\boldsymbol{U}} \boldsymbol{D}_{s}<2 \boldsymbol{I}_{M}
$$

which needs to be satisfied for the chosen $D_{s}$ in (3.4.8). With (3.2.1), this new condition is equivalent to

$$
\mathbf{0}<\boldsymbol{D}_{s} \boldsymbol{K} \boldsymbol{U}^{\top} \boldsymbol{K}^{-1} \boldsymbol{D}_{s}^{-1} \boldsymbol{D}^{*} \boldsymbol{U} \boldsymbol{K} \boldsymbol{D}_{s}<2 \boldsymbol{I}_{M}
$$

from which it can be seen that for $k_{i}$ of $\boldsymbol{K}$, there is an upper bound which depends on $\gamma_{j}, k_{j}, j=1, \ldots, i-1, d_{j}^{*}, d_{j}^{0}, j=1, \ldots, i$ and the nonzero elements of $\boldsymbol{U}$, such that the closed-loop performance is still achieved. Thus we have:

Proposition 4.3.1. The stability and asymptotic tracking performance of direct discretetime multivariable $M R A C$ designs based on the LDS decomposition of $\boldsymbol{K}_{p}$ may not be robust with respect to some reduction in actuator effectiveness.

Proof: For clarity of presentation and without loss of generality, we consider the case for $M=2$. For $\boldsymbol{K}=\boldsymbol{I}_{2}$, to ensure the desired closed-loop performance, $\boldsymbol{D}_{s}=$ 
$\operatorname{diag}\left\{\operatorname{sign}\left[d_{1}^{*}\right] \gamma_{1}, \operatorname{sign}\left[d_{2}^{*}\right] \gamma_{2}\right\}$ in (3.4.7) is chosen to satisfy (3.4.8); that is, $\gamma_{1}$ and $\gamma_{2}$ are chosen such that

$$
\begin{aligned}
& 0<\gamma_{1}<\frac{2}{d_{1}^{0}} \\
& 0<\gamma_{2}<\frac{4}{\sqrt{\left(d_{2}^{0}\right)^{2}+\frac{16 a^{2}\left|d_{1}^{0}\right|}{\alpha\left(\gamma_{1}\right)}+\left|d_{2}^{0}\right|}}
\end{aligned}
$$

with $\alpha\left(\gamma_{1}\right)=-\gamma_{1}\left(d_{1}^{0} \gamma_{1}-2\right)$ and the constant $a$ being the nonzero off-diagonal element of the $2 \times 2$ unity upper triangular matrix $\boldsymbol{U}^{1}$.

In the presence of reduction in actuator effectiveness, the performance robustness condition (4.3.1) yields

$$
\begin{gathered}
\left|d_{1}^{*}\right| k_{1} \gamma_{1}-2<0 \\
\left(\left|d_{1}^{*}\right| k_{1} \gamma_{1}-2\right)\left(\left|d_{2}^{*}\right| k_{2} \gamma_{2}-2\right)>2 a^{2}\left|d_{1}^{*}\right| \frac{k_{2}^{2} \gamma_{2}^{2}}{k_{1} \gamma_{1}}
\end{gathered}
$$

which, together with $0<k_{i} \leq 1, i=1,2$, leads to

$$
\begin{aligned}
& k_{1} \in(0,1] \\
& k_{2} \in \begin{cases}\left(0, k_{2}^{0}\right), & \text { if } 0<k_{1} \leq k_{10} \\
(0,1], & \text { if } k_{10}<k_{1} \leq 1\end{cases} \\
& k_{2}^{0}=\frac{4}{\left(\sqrt{\left(d_{2}^{*}\right)^{2}+\frac{16 a^{2}\left|d_{1}^{*}\right|}{\bar{\alpha}\left(k_{1} \gamma_{1}\right)}}+\left|d_{2}^{*}\right|\right) \gamma_{2}}
\end{aligned}
$$

with $\bar{\alpha}\left(k_{1} \gamma_{1}\right)=-k_{1} \gamma_{1}\left(\left|d_{1}^{*}\right| k_{1} \gamma_{1}-2\right)$, and

$$
k_{10}=\frac{1-\sqrt{1-\frac{2\left(\left|d_{\mid}^{*}\right| a \gamma_{2}\right)^{2}}{2-\left|d_{2}^{*}\right| \gamma_{2}}}}{\gamma_{1}\left|d_{1}^{*}\right|}
$$

We can conclude from (4.3.4)-(4.3.7) that there exists an actuation level $k_{10}$ such that if $k_{10}<k_{1} \leq 1$, the LDS based design can handle arbitrary actuator effectiveness reduction in $k_{2}$. However, for $0<k_{1} \leq k_{10}$, an upper bound $k_{2}^{0}<1$ depending

\footnotetext{
${ }^{1}$ The inequalities (4.3.2) and (4.3.3) can be obtained by solving (3.4.8) with the facts that $\boldsymbol{D}^{*}=$ $\operatorname{diag}\left\{d_{1}^{*}, d_{2}^{*}\right\}, \operatorname{sign}\left[d_{i}^{*}\right] d_{i}^{*}=\left|d_{i}^{*}\right|$, and the knowledge of $d_{i}^{0}$ such that $0<\left|d_{i}^{*}\right| \leq d_{i}^{0}, i=1,2$, from Assumption (A3.1-4D).
} 
on $k_{1}$, in addition to other design and plant parameters, is imposed on $k_{2}$ to ensure closed-loop performance. Moreover, both $k_{10}$ and $k_{2}^{0}$ depend on the unknown parameters $a$ in $\boldsymbol{U}$, and $d_{1}^{*}, d_{2}^{*}$ in $\boldsymbol{D}^{*}$ given in (2.2.10). In other words, the reduction in actuator effectiveness the LDS based design can handle, depends on the knowledge of the leading principle minors of $\boldsymbol{K}_{p}$ and the nonzero elements of $\boldsymbol{U}$. When the needed condition (4.3.1) is violated, the adaptive control system may become unstable. Therefore, the performance of the LDS based design may not be robust to some patterns of reduction in actuator effectiveness.

Remark 4.3.2. In general, explicit ranges of $k_{i}$ in which the LDS based design can still ensure the desired performance is difficult to derive from (4.3.1). Numerical methods can be used. By the Schur complement, (4.3.1) is equivalent to

$$
\left[\begin{array}{cc}
2 \boldsymbol{I}_{M} & \boldsymbol{L}_{d} \boldsymbol{D}^{*} \boldsymbol{K} \boldsymbol{U}^{\top} \\
\boldsymbol{U} \boldsymbol{K} \boldsymbol{D}^{*} \boldsymbol{L}_{d} & \boldsymbol{K} \boldsymbol{L}_{d}
\end{array}\right]>\mathbf{0}
$$

with $\boldsymbol{D}_{s}=\boldsymbol{L}_{d} \boldsymbol{D}^{*}$ chosen to satisfy (3.4.8). A linear cost function can then be constructed, and the problem is converted to the optimization of the cost function subject to the LMIs in (4.3.8) and $\mathbf{0}<\boldsymbol{K}<\boldsymbol{I}_{M}$.

Remark 4.3.3. The performance robustness properties of the LDS based design becomes manifest when $\boldsymbol{U}=\boldsymbol{I}_{M}$, i.e., $\boldsymbol{K}_{p}$ is lower triangular. The matrix inequalities in (3.4.8) and (4.3.1) can thus be decomposed into simple scalar inequalities decoupled in $\gamma_{i}$ and $k_{i}, i=1,2, \ldots, M$, respectively. To be specific, with (2.2.10) and (2.2.11), the condition in (3.4.8) leads to $0<\gamma_{i}<\frac{2}{d_{i}^{0}}$. In the presence of reduction in actuator effectiveness, the condition in (4.3.1) yields $0<k_{i}<\frac{2}{\left|d_{i}^{*}\right| \gamma_{i}}$, and we have

$$
\frac{2}{\left|d_{i}^{*}\right| \gamma_{i}}>\frac{d_{i}^{0}}{\left|d_{i}^{*}\right|} \geq 1, \quad i=1,2, \ldots, M
$$

from which we conclude that the performance of the LDS based design is robust with respect to arbitrary reduction in actuator effectiveness if $\boldsymbol{K}_{p}$ is lower triangular. 
Remark 4.3.4. The advantage of online computational efficiency of the LDS based design is at the expenses of much design effort, as the adaptation gain matrix $\boldsymbol{D}_{s}$ for (3.4.7) should be carefully chosen such that (3.4.8), which is not in explicit form of $\gamma_{i}$ and requires knowledge of $\boldsymbol{U}$, is satisfied. The choices of the adaptation gains, $\gamma_{i}$, are not straightforward even for $M=2$, as shown in (4.3.2) and (4.3.3), and require the knowledge of the off-diagonal element a of $\boldsymbol{U}$. Furthermore, the choices affect the performance robustness properties of the design.

\subsubsection{Performance Robustness of the LDU Based Design}

The controller structure based on an LDU decomposition of $\boldsymbol{K}_{p}$ is derived from (3.3.14) based on an LDS based design.

With $\Theta_{3}^{*}=\boldsymbol{K}_{p}^{-1}$ and $\boldsymbol{K}_{p}=\boldsymbol{L} \boldsymbol{D}^{*} \boldsymbol{U}$, the matching equation (3.3.12) leads to

$$
\boldsymbol{D}^{*}\left(\boldsymbol{u}-\boldsymbol{\Phi}_{0}^{*} \boldsymbol{u}-\boldsymbol{\Phi}_{1}^{* \top} \boldsymbol{\omega}_{1}-\boldsymbol{\Phi}_{2}^{* \top} \boldsymbol{\omega}_{2}-\boldsymbol{\Phi}_{20}^{*} \boldsymbol{y}-\boldsymbol{\Phi}_{3}^{*} \boldsymbol{r}\right)=\boldsymbol{L}^{-1} \boldsymbol{\xi}_{m}(z)\left[\boldsymbol{y}-\boldsymbol{y}_{m}\right]
$$

with $\boldsymbol{\Phi}_{0}^{*}=\boldsymbol{I}-\boldsymbol{U}, \boldsymbol{\Phi}_{20}^{*}=\boldsymbol{U} \boldsymbol{\Theta}_{20}^{*}, \boldsymbol{\Phi}_{i}^{*}=\boldsymbol{U} \Theta_{i}^{*}, i=1,2,3$, which motivates the controller structure

$$
\boldsymbol{u}(t)=\boldsymbol{\Phi}_{0} \boldsymbol{u}(t)+\boldsymbol{\Phi}_{1}^{\top} \boldsymbol{\omega}_{1}(t)+\boldsymbol{\Phi}_{2}^{\top} \boldsymbol{\omega}_{2}(t)+\boldsymbol{\Phi}_{20} \boldsymbol{y}(t)+\boldsymbol{\Phi}_{3} \boldsymbol{r}(t)
$$

where $\boldsymbol{\Phi}_{i}, i=0,1,2,20,3$, is the estimate of $\boldsymbol{\Phi}_{i}^{*}$.

Error Model. Denoting $\tilde{\boldsymbol{\Phi}}(t)=\boldsymbol{\Phi}(t)-\boldsymbol{\Phi}^{*}$ with $\boldsymbol{\Phi}=\left[\boldsymbol{\Phi}_{0}, \boldsymbol{\Phi}_{1}^{\top}, \boldsymbol{\Phi}_{2}^{\top}, \boldsymbol{\Phi}_{20}, \boldsymbol{\Phi}_{3}\right]^{\top}$ being the estimate of $\boldsymbol{\Phi}^{*}=\left[\boldsymbol{\Phi}_{0}^{*}, \boldsymbol{\Phi}_{1}^{* \boldsymbol{\top}}, \boldsymbol{\Phi}_{2}^{* \boldsymbol{\top}}, \boldsymbol{\Phi}_{20}^{*}, \boldsymbol{\Phi}_{3}^{*}\right]^{\boldsymbol{\top}}$, we have from (4.3.9) that

$$
\boldsymbol{\xi}_{m}(z)\left[\boldsymbol{y}-\boldsymbol{y}_{m}\right](t)+\boldsymbol{\Theta}_{0}^{*} \boldsymbol{\xi}_{m}(z)\left[\boldsymbol{y}-\boldsymbol{y}_{m}\right](t)=\boldsymbol{D}^{*} \tilde{\boldsymbol{\Phi}}^{\top}(t) \overline{\boldsymbol{\omega}}(t)
$$

where $\boldsymbol{\Theta}_{0}^{*}=\boldsymbol{L}^{-1}-\boldsymbol{I}$, and $\overline{\boldsymbol{\omega}}(t)=\left[\boldsymbol{u}^{\top}(t), \boldsymbol{\omega}^{\top}(t)\right]^{\top}$ with $\boldsymbol{\omega}(t)=\left[\boldsymbol{\omega}_{1}^{\top}(t), \boldsymbol{\omega}_{2}^{\top}(t), \boldsymbol{y}(t), \boldsymbol{r}(t)\right]^{\top}$.

Introducing the filters $h_{i}(z)=\frac{1}{f_{i}(z)}, i=1,2, \ldots, M$, with $f_{i}(z)$ being a stable polynomial of degree equal to the maximum degree of the polynomials in the first $i$ rows of $\boldsymbol{\xi}_{m}(z)$ and containing $d_{i}(z)$ (the polynomial at the $(i, i)$ position of $\boldsymbol{\xi}_{m}(z)$ ) as 
a factor, letting $\boldsymbol{\phi}_{i}^{\top}(t)$ contain the nonzero elements of the $i$ th row of $\boldsymbol{\Phi}(t), \boldsymbol{\phi}_{i}^{* \top}(t)$ contain those of $\boldsymbol{\Phi}^{*}$, and $\boldsymbol{\chi}_{i}(t)$ be the parts of the regressor $\overline{\boldsymbol{\omega}}(t)$ such that $\tilde{\boldsymbol{\Phi}}^{\top} \overline{\boldsymbol{\omega}}=$ $\left[\left(\phi_{1}-\phi_{1}^{*}\right)^{\top} \chi_{1}, \ldots,\left(\phi_{M}-\phi_{M}^{*}\right)^{\top} \chi_{M}\right]^{\top}$, denoting the parameter vectors consisting of the nonzero parameters in each row of $\boldsymbol{\Theta}_{0}^{*}$ to be $\boldsymbol{\theta}_{i}^{*}=\left[\theta_{i 1}^{*}, \ldots, \theta_{i i-1}^{*}\right]^{\top}$ and letting their estimates to be $\boldsymbol{\theta}_{i}(t)=\left[\theta_{i 1}(t), \ldots, \theta_{i i-1}(t)\right]^{\top}, i=2,3, \ldots, M$, we define the auxiliary signals $\boldsymbol{\zeta}_{i}(t)=h_{i}(z)\left[\boldsymbol{\chi}_{i}\right](t), \xi_{i}(t)=\boldsymbol{\phi}_{i}^{\top}(t) \boldsymbol{\zeta}_{i}(t)-h_{i}(z)\left[\boldsymbol{\phi}_{i}^{\top} \boldsymbol{\chi}_{i}\right](t)$, and the estimation errors to be $\epsilon_{1}(t)=\bar{e}_{1}(t)+d_{1} \xi_{1}(t)$, and $\epsilon_{i}(t)=\bar{e}_{i}(t)+\boldsymbol{\theta}_{i}^{\top}(t) \boldsymbol{\eta}_{i}(t)+d_{i} \xi_{i}(t)$, $i=2,3, \ldots, M$, where $\bar{e}_{i}$ is from the filtered tracking error vector signal $\overline{\boldsymbol{e}}(t)=$ $\boldsymbol{H}(z) \boldsymbol{\xi}_{m}(z)\left[\boldsymbol{y}-\boldsymbol{y}_{m}\right](t)=\left[\bar{e}_{1}(t), \ldots, \bar{e}_{M}(t)\right]^{\top}, d_{i}(t)$ is the estimate of $d_{i}^{*}, i=1,2, \ldots, M$, and $\boldsymbol{\zeta}_{i}(t)=h_{i}(z)\left[\boldsymbol{e}_{\theta i}\right](t)$ with $\boldsymbol{e}_{\theta i}(t)=\left[e_{\xi 1}(t), \ldots, e_{\xi i-1}(t)\right]^{\top}$ and $\boldsymbol{e}_{\xi}(t)=\boldsymbol{\xi}_{m}(z)[\boldsymbol{y}-$ $\left.\boldsymbol{y}_{m}\right](t)=\left[e_{\xi 1}, \ldots, e_{\xi M}(t)\right]^{\top}, i=2,3, \ldots, M$.

We can obtain the following error model for $i=2,3, \ldots, M$ :

$$
\begin{aligned}
& \epsilon_{1}(t)=d_{1}^{*}(t)\left(\boldsymbol{\phi}_{1}(t)-\boldsymbol{\phi}_{1}^{*}\right)^{\top} \boldsymbol{\zeta}_{1}(t)+\left(d_{1}(t)-d_{1}^{*}\right) \xi_{1}(t) \\
& \epsilon_{i}(t)=\left(\boldsymbol{\theta}_{i}(t)-\boldsymbol{\theta}_{i}^{*}\right)^{\top} \boldsymbol{\eta}_{i}(t)+d_{i}^{*}\left(\boldsymbol{\phi}_{i}(t)-\boldsymbol{\phi}_{i}^{*}\right)^{\top} \boldsymbol{\zeta}_{i}(t)+\left(d_{i}(t)-d_{i}^{*}\right) \xi_{i}(t)
\end{aligned}
$$

Adaptive Law. Based on the error model (4.3.10), the following adaptive laws are applied to update $\boldsymbol{\theta}_{i}(t), \boldsymbol{\phi}_{i}(t)$, and $d_{i}(t)$ :

$$
\begin{aligned}
& \boldsymbol{\theta}_{i}(t+1)-\boldsymbol{\theta}_{i}(t)-\frac{\boldsymbol{\Gamma}_{\theta i} \epsilon_{i}(t) \boldsymbol{\eta}_{i}(t)}{m^{2}(t)}, i=2,3, \ldots, M \\
& \boldsymbol{\phi}_{i}(t+1)-\boldsymbol{\phi}_{i}(t)=-\frac{\operatorname{sign}\left[d_{i}^{*}\right] \boldsymbol{\Gamma}_{\phi i} \epsilon_{i}(t) \boldsymbol{\zeta}_{i}(t)}{m^{2}(t)} \\
& d_{i}(t+1)-d_{i}(t)=-\frac{\gamma_{i} \epsilon_{i}(t) \xi_{i}(t)}{m^{2}(t)}, i=1,2, \ldots, M
\end{aligned}
$$

where $\mathbf{0}<\boldsymbol{\Gamma}_{\theta i}=\boldsymbol{\Gamma}_{\theta i}^{\boldsymbol{\top}}<2 \boldsymbol{I}_{M}, \mathbf{0}<\boldsymbol{\Gamma}_{\phi i}=\boldsymbol{\Gamma}_{\phi i}^{\boldsymbol{\top}}<\frac{2}{d_{i}^{0}} \boldsymbol{I}_{M}$, and $0<\gamma_{i}<2$, with $d_{i}^{0}$ as in Assumption (A3.1-4D).

The adaptive controller (4.3.9) with the adaptive laws (4.3.11) ensures closed-loop signal boundedness and asymptotic output tracking, i.e., $\lim _{t \rightarrow \infty}\left(\boldsymbol{y}(t)-\boldsymbol{y}_{m}(t)\right)=\mathbf{0}$ $[15,73]$. 
For this LDU based design, the sign and upper bound information stated in Assumptions (A3.1-1)-(A3.1-4) and (A3.1-4D) are used explicitly for the choice of adaptation gains, in contrast to the LDS based design. The parametrization of the error dynamics and the adaptation mechanism of the LDU based design are an expansion in dimension from the well-known SISO MRAC design (see Section 3.4.1), and it shares the same performance robustness property with its SISO counterpart.

Proposition 4.3.5. The stability and tracking performance of direct discrete-time multivariable MRAC designs based on the LDU decomposition of high frequency gain matrix is robust with respect to reduction in actuator effectiveness.

Proof: According to (3.2.1) and $\overline{\boldsymbol{D}}^{*}=\operatorname{diag}\left\{\bar{d}_{1}^{*}, \ldots, \bar{d}_{M}^{*}\right\}$, we have $\bar{d}_{i}^{*}=d_{i}^{*} k_{i}$. It can be seen that $\bar{d}_{i}^{*} \neq 0$ and $\operatorname{sign}\left[\bar{d}_{i}^{*}\right]=\operatorname{sign}\left[d_{i}^{*}\right]$. For the LDU based design for $\boldsymbol{K}=\boldsymbol{I}_{M}$ to ensure stability and asymptotic tracking performance under reduction in actuator effectiveness, $\left|\bar{d}_{i}^{*}\right|$ needs to satisfy $\left|\bar{d}_{i}^{*}\right| \leq d_{i}^{0}$ (to ensure the stability condition on the adaptation gain matrix), so we have $k_{i} \in\left(0, \frac{d_{i}^{0}}{\left|d_{i}^{*}\right|}\right], i=1,2, \ldots, M$. Noting that $d_{i}^{0} \geq\left|d_{i}^{*}\right|$ from Assumption (A3.1-4D), the above range for $k_{i}$ contains $(0,1]$, which implies that the closed-loop performance of the LDU based design is robust with respect to reduced actuator effectiveness.

Compared with the LDS based design, the auxiliary signal structures for the LDU based design are more complex, which need extra signal manipulations and regrouping. This effort, however, eases the design procedure, especially for the discrete-time cases, in which the choices of adaptation gains are straightforward. Besides, as an extension from the SISO discrete-time MRAC design, the LDU based design can accommodate reduced actuator effectiveness while the LDS based design cannot, in general. 


\subsection{Indirect MRAC Designs}

For indirect multivariable MRAC, the problem of nonsingular estimation of a general plant high frequency gain matrix using system input and output measurements is still a problem to be solved. Thus, we consider the indirect MRAC design for SISO systems (see Sections 3.5.1).

One key assumption in indirect SISO MRAC design is the knowledge of the sign information of the plant high frequency gain $k_{p}, \operatorname{sign}\left[k_{p}\right]$, and a lower bound $k_{p 0}$ of $\left|k_{p}\right|$ such that $0<k_{p 0} \leq\left|k_{p}\right|$, for avoiding singularity estimation of the unknown plant parameters. Based on this assumption, we obtain the following performance robustness properties:

Proposition 4.4.1. The performance of indirect MRAC designs for SISO systems may not be robust with respect to some reduction in actuator effectiveness in the sense that for the reduction greater than some level, stability and asymptotic tracking performance may no longer hold.

Proof: In the presence of reduction in actuator effectiveness, i.e., with an actuator effectiveness gain $k \in(0,1]$ at the control input, the plant high frequency gain becomes $k_{p} k$. The indirect MRAC design for $k=1$ still ensures desired closed-loop

performance if the lower bound of $\left|k_{p} k\right|$ is no less than $k_{p 0}$, i.e., $k \geq \frac{k_{p 0}}{\left|k_{p}\right|}$, which is derived from the assumption based on which a parameter projection law is designed for $k=1$. Therefore, the indirect SISO MRAC design may not be able to compensate for the reduction in actuator effectiveness with $k \in\left(0, \frac{k_{p 0}}{\left|k_{p}\right|}\right)$.

Remark 4.4.2. The performance robustness result in Proposition 4.4 .1 applies directly to the class of indirect multivariable MRAC systems presented in Section 3.5; that is, lower bounds exist for the effectiveness gains of each control channel such 
that the indirect MRAC design can adaptively compensate for reduced effectiveness not below these bounds.

\subsection{Simulation Study}

In this section we present an $M=2$ example to illustrate the performance robustness properties of the discrete-time LDS based direct MRAC design.

We consider a controlled plant (4.1.1) with $\boldsymbol{u}_{p}(t), \boldsymbol{y}(t) \in \mathbb{R}^{2}$, and

$$
\boldsymbol{G}(z)=\left[\begin{array}{cc}
\frac{1}{z-0.5} & \frac{0.6}{z+0.3} \\
\frac{1}{z+0.5} & \frac{1}{z+0.7}
\end{array}\right]
$$

which can be expressed as $\boldsymbol{G}(z)=\boldsymbol{Z}(z) \boldsymbol{P}^{-1}(z)$, where

$\boldsymbol{Z}(z)=\left[\begin{array}{cc}z+0.5 & 0.6(z+0.7) \\ z-0.5 & z+0.3\end{array}\right], \boldsymbol{P}(z)=\operatorname{diag}\{(z+0.5)(z-0.5),(z+0.3)(z+0.7)\}$

with observability index $\nu=2$. It can be verified that $\boldsymbol{G}(z)$ is minimum-phase, and $\boldsymbol{Z}(z)$ and $\boldsymbol{P}(z)$ are right coprime with $\boldsymbol{P}(z)$ column proper.

The modified interactor matrix and the associated high frequency gain matrix are

$$
\boldsymbol{\xi}_{m}(z)=\left[\begin{array}{cc}
z & 0 \\
0 & z
\end{array}\right], \quad \boldsymbol{K}_{p}=\left[\begin{array}{cc}
1 & 0.6 \\
1 & 1
\end{array}\right]
$$

from which we have $d_{1}^{*}=1, d_{2}^{*}=0.4, \operatorname{sign}\left[d_{1}^{*}\right]>0$ and $\operatorname{sign}\left[d_{2}^{*}\right]>0$. With $d_{1}^{0}=1.2$ and $d_{2}^{0}=1.0$, the standard MRAC Assumptions (A3.1-1)-(A3.1-4D) are all satisfied. Moreover, the unique LDU decomposition of $\boldsymbol{K}_{p}$ is

$$
\boldsymbol{L}=\left[\begin{array}{ll}
1 & 0 \\
1 & 1
\end{array}\right], \quad \boldsymbol{D}^{*}=\left[\begin{array}{cc}
1 & 0 \\
0 & 0.4
\end{array}\right], \quad \boldsymbol{U}=\left[\begin{array}{cc}
1 & 0.6 \\
0 & 1
\end{array}\right]
$$

The reference model is chosen to be (4.1.4) with the transfer matrix $\boldsymbol{W}_{m}(z)=$ $\boldsymbol{\xi}_{m}^{-1}(z)$ and the reference input $r(t)=[10 \cos (0.15 t), 10 \sin (0.1 t)+20 \sin (0.2 t)]^{\top}$.

The controller structure is in (3.3.14). With the specifications of $\Lambda(z)=z, \boldsymbol{\xi}_{m}(z)$, $\boldsymbol{P}(z)$, and $\boldsymbol{Z}(z)$ as above, its nominal parameter matrices $\boldsymbol{\Theta}_{1}^{*}, \boldsymbol{\Theta}_{2}^{*}, \boldsymbol{\Theta}_{20}^{*}, \boldsymbol{\Theta}_{3}^{*}=\boldsymbol{K}_{p}^{-1}$ 
can be computed from (3.3.2):

$$
\begin{aligned}
\boldsymbol{\Theta}_{1}^{*} & =\left[\begin{array}{ll}
0.3 & -1 \\
1.5 & -2
\end{array}\right], \quad \Theta_{2}^{*}=\left[\begin{array}{ll}
-0.375 & 0.375 \\
-0.525 & 0.875
\end{array}\right] \\
\boldsymbol{\Theta}_{20}^{*} & =\left[\begin{array}{cc}
-0.5 & -1.8 \\
0.5 & 3
\end{array}\right], \quad \Theta_{3}^{*}=\left[\begin{array}{cc}
2.50 & -1.5 \\
-2.5 & 2.5
\end{array}\right]
\end{aligned}
$$

With (4.5.1), $\boldsymbol{D}_{s}=\boldsymbol{I}_{2}, \boldsymbol{L}_{s}=\boldsymbol{L} \boldsymbol{D}_{s}\left(\boldsymbol{U}^{\top}\right)^{-1} \boldsymbol{D}_{s}^{-1}$, and $\boldsymbol{S}=\boldsymbol{U}^{\top} \boldsymbol{D}_{s}^{-1} \boldsymbol{D}^{*} \boldsymbol{U}$, we have

$$
\boldsymbol{\Psi}^{*}=\boldsymbol{D}_{s} \boldsymbol{S}=\left[\begin{array}{cc}
1 & 0.6 \\
0.6 & 0.76
\end{array}\right], \quad \theta_{2}^{*}=-0.4
$$

The adaptive laws are (3.4.7) with $\boldsymbol{D}_{s}$ chosen to be $\boldsymbol{D}_{s}=\operatorname{diag}\left[\gamma_{1}, \gamma_{2}\right]=\boldsymbol{I}_{2}$ satisfying the design condition (3.4.8), and in the estimation error model, $f(z)$ is chosen as $f(z)=z$. The initial estimates of $\theta_{2}^{*}, \boldsymbol{\Theta}^{*}, \Psi^{*}$ are taken to be $\theta_{2}(0)=0.95 \theta_{2}^{*}$, $\boldsymbol{\Theta}(0)=0.95 \boldsymbol{\Theta}^{*}$, and $\boldsymbol{\Psi}(0)=0.8 \boldsymbol{\Psi}^{*}$.

From (4.3.7) we obtain $k_{10}=0.2584$, and by (4.3.5), we know that for a reduction in actuator effectiveness of the first control channel such that $k_{1}>k_{10}$, the performance is robust with respect to arbitrary $k_{2} \in(0,1]$. This is shown in Fig. 4.2 where the tracking error approaches zero asymptotically for $\boldsymbol{K}=\operatorname{diag}\{0.4,0.6\}$. Furthermore, for a $k_{1} \leq k_{10}$, the stability and asymptotic tracking performance may not hold for some $k_{2}$. As is shown in Fig. 4.3, with $k_{1}=0.1<k_{10}$, for $k_{2}=1.0$, the asymptotic tracking performance is lost (simulation was performed for a much longer time, and only the first 1000 steps are shown here for clarity). However, as shown in Fig. 4.4, asymptotic tracking is achieved for $\boldsymbol{K}=\operatorname{diag}\{0.25,0.65\}$, as $0.65<k_{2}^{0}=0.8043$ from (4.3.6).

The simulation results illustrate the main features of the performance robustness of the LDS based design.

This chapter focuses on the performance robustness properties of MRAC with respect to reduced actuator effectiveness, the type of actuator failure in which partial control surface is damaged and the actuator effectiveness reduces to a fraction 

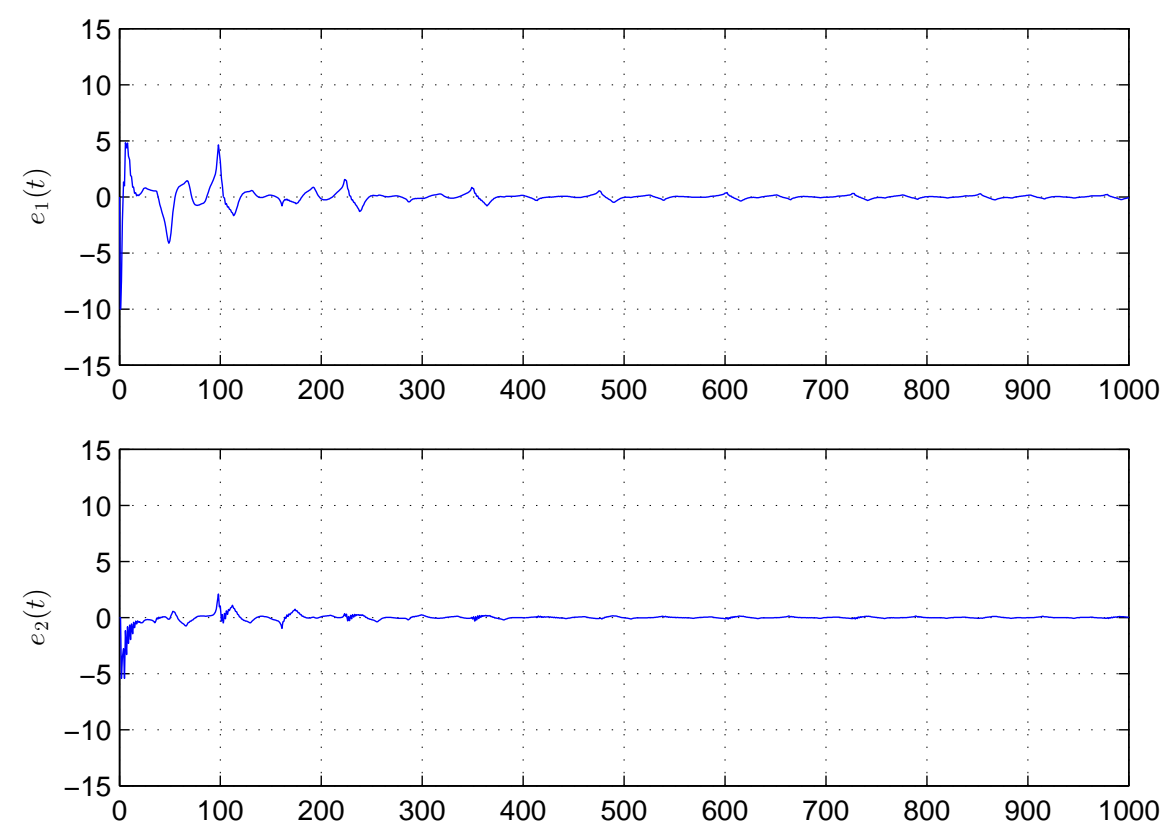

Figure 4.2: Tracking error $e(t)$ for $K=\operatorname{diag}\{0.4,0.6\}$.

of the normal level (that without reduction in effectiveness). We conduct the performance robustness analysis of some MRAC schemes with respect to reduced actuator effectiveness, which can be represented by an actuator effectiveness matrix at the control inputs. We determine whether or not an MRAC scheme designed for the normal case, i.e., that without reduction in actuator effectiveness, can still achieve the desired closed-loop stability and asymptotic tracking performance in the presence of reduction in effectiveness. The analytical gain margin results of MRAC systems in Chapter 3 are applied for solutions of the performance robustness problem in the presence of reduced actuator effectiveness. 

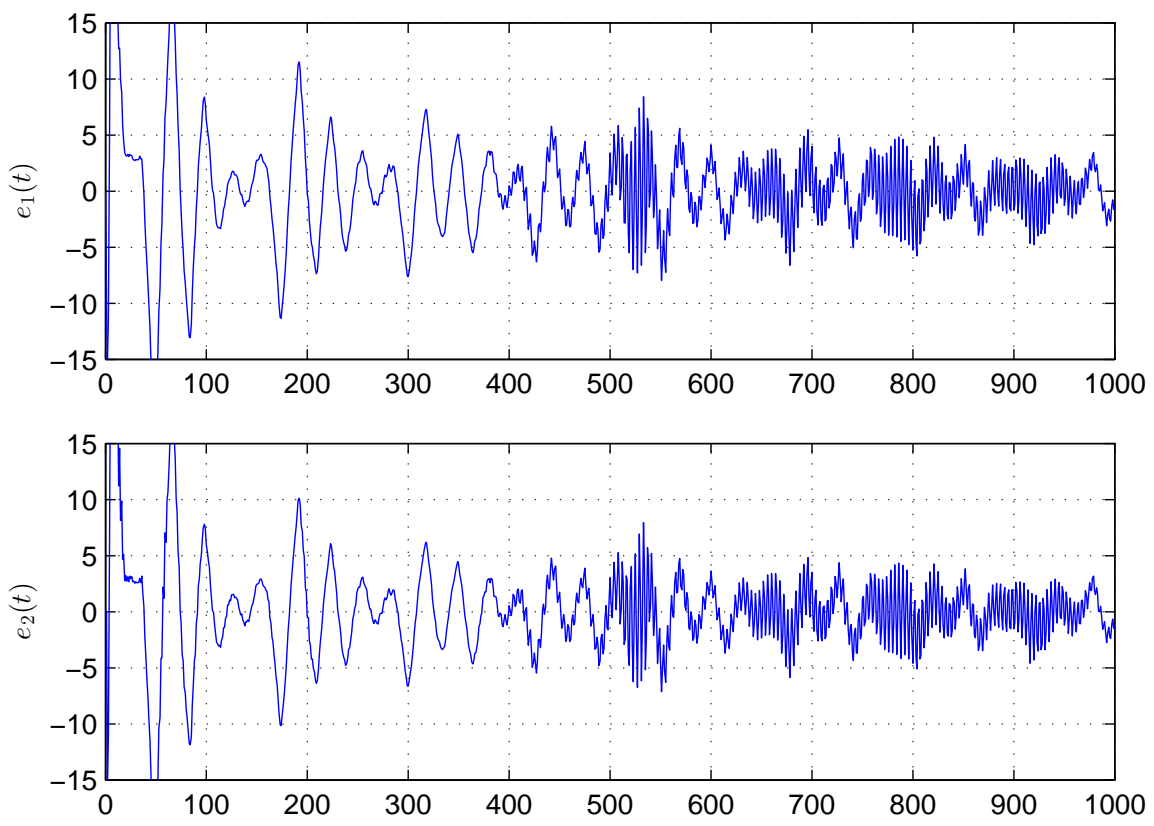

Figure 4.3: Tracking error $e(t)$ for $K=\operatorname{diag}\{0.1,1.0\}$.
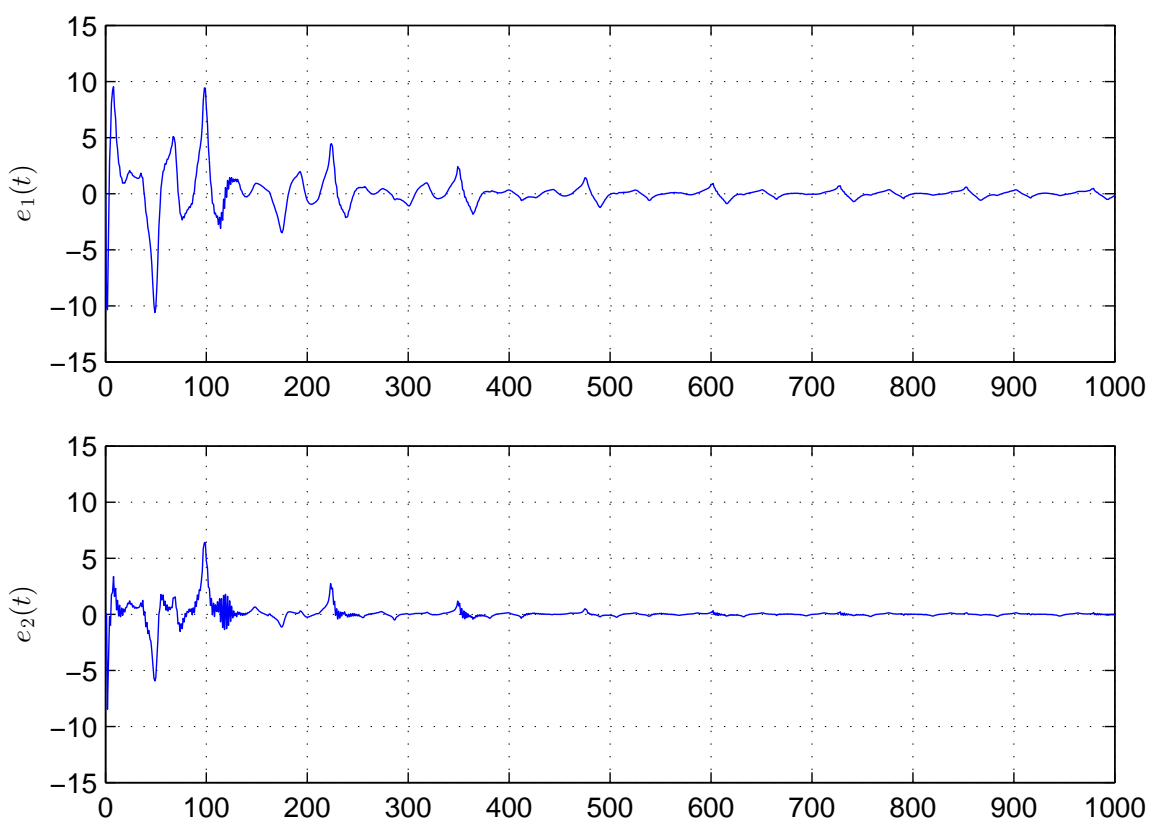

Figure 4.4: Tracking error $e(t)$ for $K=\operatorname{diag}\{0.25,0.65\}$. 


\section{Chapter 5}

\section{Adaptive Control of Piecewise Linear Systems with State Feedback for State Tracking}

In this chapter, the adaptive state feedback for state tracking (SFST) control problem is studied for piecewise linear systems. Piecewise linear reference model systems are used for generating desired state trajectories and their stability properties are investigated. Adaptive state feedback control schemes are developed, and their stability and tracking performance are analyzed and evaluated by simulation examples.

The chapter is organized as follows. The state tracking control problem for piecewise linear systems is stated in Section 5.1. Stability properties of piecewise linear reference model systems are studied in Section 5.2. Adaptive control designs for singleinput systems are proposed and analyzed in Section 5.3, and multivariable designs are presented in Section 5.4. Illustrative examples to demonstrate the effectiveness of the proposed adaptive control schemes are provided in Section 5.5. 


\subsection{Problem Statement}

Consider the piecewise linear system:

$$
\begin{aligned}
& \dot{\boldsymbol{x}}(t)=\boldsymbol{A}(t) \boldsymbol{x}(t)+\boldsymbol{B}(t) \boldsymbol{u}(t), \quad \boldsymbol{x}\left(t_{0}\right)=\boldsymbol{x}_{0} \\
& \boldsymbol{y}(t)=\boldsymbol{C}^{\boldsymbol{\top}} \boldsymbol{x}(t)
\end{aligned}
$$

where $\boldsymbol{x}(t) \in \mathbb{R}^{n}$ is the state vector, $\boldsymbol{y}(t) \in \mathbb{R}^{M}$ is the system output, $\boldsymbol{u}(t) \in \mathbb{R}^{M}$ is the control input to be specified by an adaptive control law. The plant parameter matrices $\boldsymbol{A}(t) \in \mathbb{R}^{n \times n}$ and $\boldsymbol{B}(t) \in \mathbb{R}^{n \times M}$ are unknown, time-varying (piecewise constant) that can be expressed as

$$
\boldsymbol{A}(t)=\sum_{i=1}^{l} \boldsymbol{A}_{i} \chi_{i}(t), \quad \boldsymbol{B}(t)=\sum_{i=1}^{l} \boldsymbol{B}_{i} \chi_{i}(t)
$$

where the unknown parameter matrix set $\left(\boldsymbol{A}_{i}, \boldsymbol{B}_{i}\right)$ describes the $i$ th subsystem, and $\chi_{i}(t), i=1,2, \ldots, M$, are the indicator functions that characterize subsystem switches and are defined as

$$
\chi_{i}(t)= \begin{cases}1, & \text { if }(\boldsymbol{A}(t), \boldsymbol{B}(t))=\left(\boldsymbol{A}_{i}, \boldsymbol{B}_{i}\right) \\ 0, & \text { otherwise }\end{cases}
$$

Since at each specific time instant $t$, only one subsystem is active, the indicator functions defined as above have the following properties

$$
\sum_{i=1}^{l} \chi_{i}(t)=1, \quad \chi_{j}(t) \chi_{k}(t)=0, \quad j \neq k, \quad j, k \in \mathcal{I}
$$

Control Objective. The control objective is to design an adaptive state feedback control law $\boldsymbol{u}(t)$ for closed-loop stability and asymptotic tracking by the piecewise linear system state $\boldsymbol{x}(t)$ of a reference trajectory $\boldsymbol{x}_{m}(t)$, generated from a reference model system. In this case, the system state $\boldsymbol{x}(t)$ is assumed to be available for measurement.

Next, we will first present in Section 5.3 a SFST MRAC design for the piecewise linear systems (5.1.1) with single input, i.e., $\boldsymbol{u}(t)=u(t) \in \mathbb{R}$. We then propose 
a multivariate adaptive state feedback design for the piecewise linear systems with dynamics offsets (2.1.6) in Section 5.4.

\subsection{Time-Varying Reference Model Systems}

A reference trajectory $\boldsymbol{x}_{m}(t)$ should be specified representing the desired system behaviors for each subsystem and the transitions in between. Such a reference trajectory may be specified locally for each subsystem, which is then pieced together to form $\boldsymbol{x}_{m}(t)$. Here, we present a reference model system which is also piecewise linear, for the single-input case. Conditions for its stability are also applicable to the multivariable case.

\subsubsection{Piecewise Linear Reference Model System}

We consider a piecewise linear reference model system

$$
\begin{aligned}
& \dot{\boldsymbol{x}}_{m}(t)=\boldsymbol{A}_{m}(t) \boldsymbol{x}_{m}(t)+\boldsymbol{b}_{m}(t) r(t) \\
& \boldsymbol{A}_{m}(t)=\sum_{i=1}^{l} \boldsymbol{A}_{m i} \chi_{i}(t), \quad \boldsymbol{b}_{m}(t)=\sum_{i=1}^{l} \boldsymbol{b}_{m i} \chi_{i}(t)
\end{aligned}
$$

where $\boldsymbol{A}_{m i} \in \mathbb{R}^{n \times n}$ are stable, $\boldsymbol{b}_{m i} \in \mathbb{R}^{n}, i \in \mathcal{I}$, and $r(t) \in \mathbb{R}$ is a bounded, piecewise continuous reference input signal. When the $i$ th subsystem, $\left(\boldsymbol{A}_{m i}, \boldsymbol{b}_{m i}\right)$, is active, as indicated by $\chi_{i}(t)=1$, the state trajectory $\boldsymbol{x}_{m}(t)$ is desirable for $\boldsymbol{x}(t)$ to follow.

\subsubsection{Stability of Piecewise Linear Systems}

The boundedness of $\boldsymbol{x}_{m}(t)$ is a prerequisite in a tracking problem. Let the increasing sequence $\left\{t_{k}\right\}_{k=1}^{\infty}$ denote the time instants at which subsystem switches. To generate $\boldsymbol{x}_{m}(t)$, the reference model system (5.2.1) switches at the same time, while in between two successive switches, it is governed by a time-invariant parameter set $\left(\boldsymbol{A}_{m i}, \boldsymbol{b}_{m i}\right)$. 
Exponential stability of its homogeneous system,

$$
\dot{\boldsymbol{z}}(t)=\boldsymbol{A}_{m}(t) \boldsymbol{z}(t), \quad \boldsymbol{A}_{m}(t)=\sum_{i=1}^{l} \boldsymbol{A}_{m i} \chi_{i}(t)
$$

is sufficient for stability of (5.2.1), which has been studied in $[19,29,39,47]$. It is well known that for the homogeneous system (5.2.2) with each $\boldsymbol{A}_{m i}$ stable to be exponentially stable, the time interval between two consecutive switches should be long enough. Let $T_{0}$ denote the minimum switching time interval, i.e., $T_{0}=$ $\min _{k \in \mathbb{Z}^{+}}\left\{t_{k}-t_{k-1}\right\}$, where $\mathbb{Z}^{+}$stands for all positive integers, and $\boldsymbol{P}_{m i}, \boldsymbol{Q}_{m i} \in \mathbb{R}^{n \times n}$ be symmetric, positive definite satisfying

$$
\boldsymbol{A}_{m i}^{\top} \boldsymbol{P}_{m i}+\boldsymbol{P}_{m i} \boldsymbol{A}_{m i}=-\boldsymbol{Q}_{m i}, \quad i \in \mathcal{I}
$$

Due to the stability of $\boldsymbol{A}_{m i}$, there exist $a_{m i}, \lambda_{m i}>0$ such that $\left\|e^{\boldsymbol{A}_{m i} t}\right\| \leq a_{m i} e^{-\lambda_{m i} t}$. Define $a_{m}=\max _{i \in \mathcal{I}} a_{m i}, \lambda_{m}=\min _{i \in \mathcal{I}} \lambda_{m i}$, and with $\lambda_{\min }[\cdot]$ and $\lambda_{\max }[\cdot]$ denoting the minimum and maximum eigenvalues of a matrix, $\alpha=\max _{i \in \mathcal{I}} \lambda_{\max }\left[\boldsymbol{P}_{m i}\right]$, $\beta=\min _{i \in \mathcal{I}} \lambda_{\min }\left[\boldsymbol{P}_{m i}\right]$. The following lemma gives a lower bound on $T_{0}$ that ensures exponential stability of the homogeneous system, thus the stability of (5.2.1):

Lemma 5.2.1. The system (5.2.2) is exponentially stable with decay rate $\sigma \in(0,1 / 2 \alpha)$ if $T_{0}$ satisfies

$$
T_{0} \geq \frac{\alpha}{1-2 \sigma \alpha} \ln \left(1+\mu \Delta_{\boldsymbol{A}_{m}}\right), \quad \mu=\frac{a_{m}^{2}}{\lambda_{m} \beta} \max _{i \in \mathcal{I}}\left\|\boldsymbol{P}_{m i}\right\|
$$

where $\Delta_{\boldsymbol{A}_{m}}$ stands for the largest difference between any two subsystem matrices of $\boldsymbol{A}_{m}(t)$, i.e., $\Delta_{\boldsymbol{A}_{m}}=\max _{i, j \in \mathcal{I}}\left\|\boldsymbol{A}_{m i}-\boldsymbol{A}_{m j}\right\|$.

Proof: The proof of this lemma is provided in the Appendix (see Section 9.2).

Remark 5.2.2. Unlike typical slow-switching conditions in the literature (cf. [38, Lemma 2]), (5.2.4) relates a lower bound of the minimum switching time interval 
required for the stability of (5.2.1) to the largest difference between any two subsystems, i.e., $\Delta_{\boldsymbol{A}_{m}}$. As $\Delta_{\boldsymbol{A}_{m}}$ reduces to zero, this lower bound decreases and approaches zero as well, which as shown next reverts to the results in [60]; that is, for arbitrary subsystem switches, the closed-loop stability and asymptotic state tracking performance follow, when all subsystem are matched to a linear time-invariant reference model system. The same conclusion can be drawn when there exists a common Lyapunov matrix $\boldsymbol{P}_{m}$ such that $\boldsymbol{A}_{m i}^{\top} \boldsymbol{P}_{m}+\boldsymbol{P}_{m} \boldsymbol{A}_{m i}<\mathbf{0}$.

\subsection{Design for Single-Input Systems}

A new adaptive state feedback controller structure is proposed for the piecewise linear system (5.1.1) with single input, i.e.,

$$
\begin{aligned}
\dot{\boldsymbol{x}}(t) & =\boldsymbol{A}(t) \boldsymbol{x}(t)+\boldsymbol{b}(t) u(t), \quad u(t) \in \mathbb{R} \\
\boldsymbol{A}(t) & =\sum_{i=1}^{l} \boldsymbol{A}_{i} \chi_{i}(t), \quad \boldsymbol{b}(t)=\sum_{i=1}^{l} \boldsymbol{b}_{i} \chi_{i}(t)
\end{aligned}
$$

where $\boldsymbol{A}_{i}$ and $\boldsymbol{b}_{i}$ are unknown, and $\chi_{i}(t)$ are the indicator functions, taking values of either 1 or 0 independent of $\boldsymbol{x}(t), u(t)$, and defined to satisfy (5.1.3) and (5.1.4).

\subsubsection{Controller Structure}

The following adaptive state feedback controller structure is applied:

$$
u(t)=\boldsymbol{k}_{x}^{\top}(t) \boldsymbol{x}(t)+k_{r}(t) r(t)
$$

where $r(t)$ is the reference input signal as defined in (5.2.1), and the parameter vectors $\boldsymbol{k}_{x}(t)=\sum_{i=1}^{l} \boldsymbol{k}_{x i}(t) \chi_{i}(t), k_{r}(t)=\sum_{i=1}^{l} k_{r i}(t) \chi_{i}(t)$ are the time-varying estimates of the nominal controller parameters $\boldsymbol{k}_{x}^{*}(t)=\sum_{i=1}^{l} \boldsymbol{k}_{x i}^{*} \chi_{i}(t)$ and $k_{r}^{*}(t)=\sum_{i=1}^{l} k_{r i}^{*} \chi_{i}(t)$, with $\boldsymbol{k}_{x i}^{*}, k_{r i}^{*}, i \in \mathcal{I}$, defined to satisfy the following assumption: 
(A5.3-1) Nominal parameter vectors $\boldsymbol{k}_{x i}^{*}, k_{r i}^{*}$ exist such that

$$
\boldsymbol{A}_{m i}=\boldsymbol{A}_{i}+\boldsymbol{b}_{i} \boldsymbol{k}_{x i}^{* \top}, \quad \boldsymbol{b}_{m i}=\boldsymbol{b}_{i} k_{r i}^{*}
$$

With (5.3.2) in (5.3.1) under Assumption (A5.3-1), the closed-loop system becomes

$$
\dot{\boldsymbol{x}}=\boldsymbol{A}_{m} \boldsymbol{x}+\boldsymbol{b}_{m} r+\sum_{i=1}^{l} \boldsymbol{b}_{i} \tilde{\boldsymbol{k}}_{x i}^{\top} \chi_{i} \boldsymbol{x}+\sum_{i=1}^{l} \boldsymbol{b}_{i} \tilde{k}_{r i} \chi_{i} r
$$

where $\tilde{\boldsymbol{k}}_{x}(t)=\boldsymbol{k}_{x}(t)-\boldsymbol{k}_{x}^{*}(t), \tilde{k}_{r}(t)=k_{r}(t)-k_{r}^{*}(t), \tilde{\boldsymbol{k}}_{x i}(t)=\boldsymbol{k}_{x i}(t)-\boldsymbol{k}_{x i}^{*}$, and $\tilde{k}_{r i}(t)=$ $k_{r i}(t)-k_{r i}^{*}$.

The equations in (5.3.3) are the plant-model matching conditions; that is, if the plant parameter matrices $\boldsymbol{A}_{i}, \boldsymbol{b}_{i}$ are known, the nominal control law

$$
u(t)=\boldsymbol{k}_{x}^{* \top}(t) \boldsymbol{x}(t)+k_{r}^{*}(t) r(t)
$$

leads to

$$
\dot{\boldsymbol{e}}(t)=\boldsymbol{A}_{m}(t) \boldsymbol{e}(t), \quad \boldsymbol{e}(t)=\boldsymbol{x}(t)-\boldsymbol{x}_{m}(t)
$$

with $\boldsymbol{e}(t)$ approaching zero exponentially, as a result of Lemma 5.2.1. In addition, all the signals are bounded, so that the control objective is achieved.

\subsubsection{Adaptive Laws}

To develop adaptive laws for updating the parameter estimates $\boldsymbol{k}_{x}(t), k_{r}(t)$, an error

equation in terms of $\boldsymbol{e}(t), \tilde{\boldsymbol{k}}_{x}(t)$, and $\tilde{k}_{r}(t)$ is needed. In view of (5.2.1) and (5.3.4), we have

$$
\dot{\boldsymbol{e}}=\sum_{i=1}^{l}\left[\boldsymbol{A}_{m i} \chi_{i} \boldsymbol{e}+\frac{1}{k_{r i}^{*}} \boldsymbol{b}_{m i}\left(\tilde{\boldsymbol{k}}_{x i}^{\top} \chi_{i} \boldsymbol{x}+\tilde{k}_{r i} \chi_{i} r\right)\right]
$$

based on which parameter projection adaptive laws are proposed next with stability and tracking performance analyzed. 
With the knowledge of lower and upper bounds of the parameters in $\boldsymbol{k}_{x i}^{*}, k_{r i}^{*}$, the parameter projection adaptive laws

$$
\begin{aligned}
& \dot{\boldsymbol{k}}_{x i}(t)=-\operatorname{sign}\left[k_{r i}^{*}\right] \boldsymbol{\Gamma}_{i} \boldsymbol{x}(t) \boldsymbol{e}^{\boldsymbol{\top}}(t) \boldsymbol{P}_{m i} \boldsymbol{b}_{m i} \chi_{i}(t)+\boldsymbol{f}_{x i}(t) \chi_{i}(t) \\
& \dot{k}_{r i}(t)=-\operatorname{sign}\left[k_{r i}^{*}\right] \gamma_{i} r(t) \boldsymbol{e}^{\boldsymbol{\top}}(t) \boldsymbol{P}_{m i} \boldsymbol{b}_{m i} \chi_{i}(t)+f_{r i}(t) \chi_{i}(t)
\end{aligned}
$$

are applied to update the controller parameters in (5.3.2), where $\boldsymbol{\Gamma}_{i}>\mathbf{0}$ and is diagonal, $\gamma_{i}>0$, and $\boldsymbol{P}_{m i}=\boldsymbol{P}_{m i}^{\top}>0, i \in \mathcal{I}$, satisfy (5.2.3) for some $\boldsymbol{Q}_{m i}=\boldsymbol{Q}_{m i}^{\top}>\mathbf{0}$. Here we assume:

(A5.3-2) $\operatorname{sign}\left[k_{r i}^{*}\right]$, the sign of $k_{r i}^{*}, i \in \mathcal{I}$, are known.

The initial estimate of each element in $\boldsymbol{k}_{x}(t), k_{r}(t)$ is chosen to be within its known bounds. The projection terms $\boldsymbol{f}_{x i}(t), f_{r i}(t)$ are so defined as to confine the parameter estimates inside these bounds for all time: parameter adaptation is active (i.e., $\boldsymbol{f}_{x i}(t)=\mathbf{0}, f_{r i}(t)=0$ ) when the estimates are within those bounds, while it is deactivated otherwise, and the estimates are left unchanged (i.e., $\boldsymbol{k}_{x i}(t)=\mathbf{0}$, $\left.\dot{k}_{r i}(t)=0\right)$.

\subsubsection{Stability and Tracking Properties}

Let $\left\{t_{k}\right\}_{k=1}^{\infty}$ denote the time instants at which (5.3.1) switches, and $T_{0}=\min _{k \in \mathbb{Z}^{+}}\left\{t_{k}-\right.$ $\left.t_{k-1}\right\}$ be the minimum switching time interval. With the definitions of $a_{m}, \lambda_{m}, \alpha, \beta$, $\mu, \Delta_{\boldsymbol{A}_{m}}$ in Lemma 5.2.1, we have the following stability and tracking properties:

Theorem 5.3.1. Consider the closed-loop system consisting of the piecewise linear system (5.3.1), the reference model system (5.2.1), and the controller (5.3.2) updated by the adaptive laws (5.3.6). If

$$
T_{0} \geq T_{d}=\alpha(1+\kappa) \ln \left(1+\mu \Delta_{\boldsymbol{A}_{m}}\right), \quad \kappa>0
$$


then all closed-loop signals are bounded, and the tracking error $\boldsymbol{e}(t)$ is small in the sense that

$$
\int_{t}^{t+T} \boldsymbol{e}^{\top}(\tau) \boldsymbol{e}(\tau) d \tau \leq \mu \Delta_{\boldsymbol{A}_{m}} c_{0} \frac{T}{T_{0}}+c_{1}, \quad \forall t \geq t_{0}, \quad \forall T>0
$$

with $c_{1}=\left(1+\mu \Delta_{\boldsymbol{A}_{m}}\right) c_{0}$, for some $c_{0}>0$.

Proof: The proof of this theorem is given in Section 9.3.

The parameter projection adaptive control scheme ensures signal boundedness and small tracking error in the mean-square sense provided that the switching time interval is large enough. Asymptotic tracking performance is restored if $\Delta_{\boldsymbol{A}_{m}}=0$, as stated in the following corollary:

Corollary 5.3.2. When $\Delta_{\boldsymbol{A}_{m}}=0$, that is, all subsystems are matched to an LTI reference model system according to (5.3.3) for $\boldsymbol{A}_{m i}=\boldsymbol{A}_{m}, i \in \mathcal{I}$, closed-loop stability and asymptotic tracking are achieved for arbitrary subsystem switches.

Proof: Closed-loop stability follows directly from Theorem 5.3.1 for $T_{d}=0$, i.e., arbitrary subsystem switches. As a result of (5.3.7), $\int_{0}^{\infty} \boldsymbol{e}^{\top}(\tau) \boldsymbol{e}(\tau) d \tau \leq c_{1}<\infty$, i.e., $\boldsymbol{e}(t) \in \mathcal{L}^{2}$. From (5.3.5) and signal boundedness, it follows that $\dot{\boldsymbol{e}}(t) \in \mathcal{L}^{\infty}$, and therefore, $\lim _{t \rightarrow \infty} \boldsymbol{e}(t)=\mathbf{0}$.

Subsystem switches lead to instantaneous increases (jumps) in the Lyapunov function $V: V\left(t_{k}\right)-V\left(t_{k}^{-}\right)$. To ensure that they do not lead to instability, parameter projection is indispensable in the adaptive laws (5.3.6). With parameter projection, a uniform bound $c_{p}$ (see Section 9.3), together with a sufficiently large switching time interval $\left(T_{0} \geq T_{d}\right)$, ensures $V$ be below $l c_{p}\left(1+\mu \Delta_{\boldsymbol{A}_{m}}\right)(1+\kappa) / \kappa$ or $V\left(t_{k-1}\right)$, before a subsequent switch occurs. A bound $c_{0}$ on $V$ can be established, and closed-loop stability follows. However, it is not necessary when additional conditions (persistency 
of excitation) are imposed on the system (studied in the next subsection), or when subsystem switches do not cause jumps in $V$, as summarized below:

Corollary 5.3.3. If a common Lyapunov matrix $\boldsymbol{P}_{m}$ exists for $\boldsymbol{A}_{m i}, i \in \mathcal{I}$, of the reference model system (5.2.1) such that

$$
\boldsymbol{A}_{m i}^{\top} \boldsymbol{P}_{m}+\boldsymbol{P}_{m} \boldsymbol{A}_{m i}<-\boldsymbol{Q}_{m}, \quad \boldsymbol{Q}_{m}^{\top}=\boldsymbol{Q}_{m}>\mathbf{0}
$$

then the closed-loop system with (5.3.2) updated by the adaptive laws

$$
\begin{gathered}
\dot{\boldsymbol{k}}_{x i}(t)=-\operatorname{sign}\left[k_{r i}^{*}\right] \boldsymbol{\Gamma}_{i} \boldsymbol{x}(t) \boldsymbol{e}^{\boldsymbol{\top}}(t) \boldsymbol{P}_{m} \boldsymbol{b}_{m i} \chi_{i}(t) \\
\dot{k}_{r i}(t)=-\operatorname{sign}\left[k_{r i}^{*}\right] \gamma_{i} r(t) \boldsymbol{e}^{\boldsymbol{\top}}(t) \boldsymbol{P}_{m} \boldsymbol{b}_{m i} \chi_{i}(t)
\end{gathered}
$$

ensures closed-loop signal boundedness and asymptotic state tracking performance, i.e., $\lim _{t \rightarrow \infty} \boldsymbol{e}(t)=\mathbf{0}$, for arbitrary subsystem switches.

$\underline{\text { Proof: }}$ With a common Lyapunov matrix $\boldsymbol{P}_{m}$, the piecewise Lyapunov function (9.3.1) reduces to

$$
V=\boldsymbol{e}^{\top} \boldsymbol{P}_{m} \boldsymbol{e}+\sum_{i=1}^{l} \frac{1}{\left|k_{r i}^{*}\right|}\left(\tilde{\boldsymbol{k}}_{x i}^{\top} \boldsymbol{\Gamma}_{i}^{-1} \tilde{\boldsymbol{k}}_{x i}+\gamma_{i}^{-1} \tilde{k}_{r i}^{2}\right)
$$

which is continuous, and its time derivative satisfies

$$
\dot{V} \leq-\boldsymbol{e}^{\top} \boldsymbol{Q}_{m} \boldsymbol{e}
$$

Hence $\boldsymbol{e}(t) \in \mathcal{L}^{2}$ and closed-loop stability can be concluded for arbitrary subsystem switches. From (5.3.5) and signal boundedness, it follows that $\dot{\boldsymbol{e}}(t) \in \mathcal{L}^{\infty}$, and therefore, $\lim _{t \rightarrow \infty} \boldsymbol{e}(t)=\mathbf{0}$.

Remark 5.3.4. The case for an LTI reference model system (Corollary 5.3.2) is a special case of the existence of a common $\boldsymbol{P}_{m}$ matrix for a piecewise linear reference model system (Corollary 5.3.2) with $\Delta_{\boldsymbol{A}_{m}}=0$, such that the parameter projection terms in (5.3.6) may be removed without affecting the closed-loop stability and tracking performance. 
It is known that only $\boldsymbol{A}_{m i}$ with special structures, e.g., those commuting pairwise [30] or being simultaneously transformable into triangular form [31], have a common $\boldsymbol{P}_{m}$ matrix. When no such $\boldsymbol{P}_{m}$ exists, additional conditions are needed to achieve asymptotic tracking performance, as studied next.

\subsubsection{Adaptation with Sufficiently Rich Reference Input}

The control law (5.3.2) with nominal parameters defined to satisfy the matching condition (5.3.3) results in exponential convergence of $\boldsymbol{e}(t)$ for subsystem switches that are sufficiently slow, due to the fact that each subsystem in closed loop is exponentially stable. In the adaptive control problem, if some of the subsystem with indices $i \in$ $\mathcal{I}^{*} \subset \mathcal{I}$ are no longer active after a finite time $T_{i} \geq t_{0}$, while other subsystem are active intermittently over infinitely many intervals, then under the persistency of excitation (P.E.) condition, the following stability and tracking properties follow for $t \geq T^{*}=\max _{i \in \mathcal{I}^{*}}\left\{T_{i}\right\}:$

Theorem 5.3.5. Consider the closed-loop system consisting of the piecewise linear system (5.3.1), the piecewise linear reference model system (5.2.1), and the controller (5.3.2) updated by the adaptive laws:

$$
\begin{aligned}
& \dot{\boldsymbol{k}}_{x i}(t)=-\operatorname{sign}\left[k_{r i}^{*}\right] \boldsymbol{\Gamma}_{i} \boldsymbol{x}(t) \boldsymbol{e}^{\top}(t) \boldsymbol{P}_{m i} \boldsymbol{b}_{m i} \chi_{i}(t) \\
& \dot{k}_{r i}(t)=-\operatorname{sign}\left[k_{r i}^{*}\right] \gamma_{i} r(t) \boldsymbol{e}^{\top}(t) \boldsymbol{P}_{m i} \boldsymbol{b}_{m i} \chi_{i}(t)
\end{aligned}
$$

Suppose the reference input signal $r(t)$ is sufficiently rich of order $n+1$ (i.e., $r(t)$ has at least $n+1$ different frequencies) and $\left(\boldsymbol{A}_{m i}, \boldsymbol{b}_{m i}\right)$ are controllable, $i \in \mathcal{I}$. If the switching time intervals are sufficiently large, then all closed-loop signals are bounded for $t \geq t_{0} ; \boldsymbol{e}(t), \tilde{\boldsymbol{k}}_{x i}(t), \tilde{k}_{r i}(t)$ converge to zero exponentially, $i \in \mathcal{I}-\mathcal{I}^{*}, t \geq T^{*}$; and $\boldsymbol{k}_{x i}(t)=\boldsymbol{k}_{x i}\left(T_{i}\right), k_{r i}(t)=k_{r i}\left(T_{i}\right), i \in \mathcal{I}^{*}, t \geq T_{i}$.

Proof: The proof of Theorem 5.3.5 is provided in Section 9.4. 
Remark 5.3.6. If the parameter projection adaptive laws (5.3.6) are applied instead of (5.3.8), the time derivative of the Lyapunov-like function in (9.3.1) becomes

$$
\dot{V}=\boldsymbol{z}^{\top}\left(\boldsymbol{A}_{0}^{\top} \boldsymbol{P}_{0}+\boldsymbol{P}_{0} \boldsymbol{A}_{0}\right) \boldsymbol{z}+\frac{1}{\left\|\boldsymbol{b}_{i}\right\|^{2}\left|k_{r i}^{*}\right|}\left(\sum_{j=1}^{n} \gamma_{i j}^{-1} \tilde{k}_{x i j}^{\top} f_{x i j}+\gamma_{i}^{-1} \tilde{k}_{r i} f_{r i}\right)
$$

Since $\tilde{k}_{x i j}(t) f_{x i j}(t) \leq 0$ and $\tilde{k}_{r i}(t) f_{r i}(t) \leq 0$, the condition (9.4.5) is satisfied, and conclusions of Theorem 5.3.5 are still valid. See Section 9.4 for details.

\subsubsection{Simulation Study}

Simulations are performed to demonstrate the system stability and tracking performance with the proposed adaptive control schemes applied to the piecewise linear system model of the longitudinal dynamics of the NASA GTM [82] at multiple operating points.

For simplicity of presentation, we choose $l=2$, and trim the GTM at steadystate, straight, wings-level flight conditions at 70 knots and 80 knots at $800 \mathrm{ft}$., respectively, for a piecewise linear longitudinal model in the form of (5.3.1), where $\boldsymbol{x}=[u, w, q, \theta]^{\top}$ with the elements being the perturbed aircraft velocity component along the $\mathrm{x}$ - and z-body-axis (fps), angular velocity along the y-body-axis ( $\mathrm{rad} / \mathrm{s})$, and pitch angle (rad), respectively, and $u(t)$ is the perturbed elevator deflection $\delta_{e}$. The reference model system (5.2.1) is chosen to be the nominal closed-loop system with poles placed at $-1,-2,-3,-4$, such that the plant-model matching condition (5.3.3) is satisfied, and $\boldsymbol{b}_{m i}=\boldsymbol{b}_{i}, i=1,2$. The matrices $\boldsymbol{P}_{m i}$ are then obtained by solving the Lyapunov equations (5.2.3) with $\boldsymbol{Q}_{m i}=\boldsymbol{I}_{4}, i=1,2$.

Simulations are performed for two cases, for the reference input signal $r(t)$ not sufficiently rich and sufficiently rich, respectively. In each case, the initial conditions are $\boldsymbol{x}_{m}(0)=[7,-4,0.1,0.5]^{\top}, \boldsymbol{x}(0)=\mathbf{0}$, and the initial parameter estimates are $90 \%$ of their nominal values. 

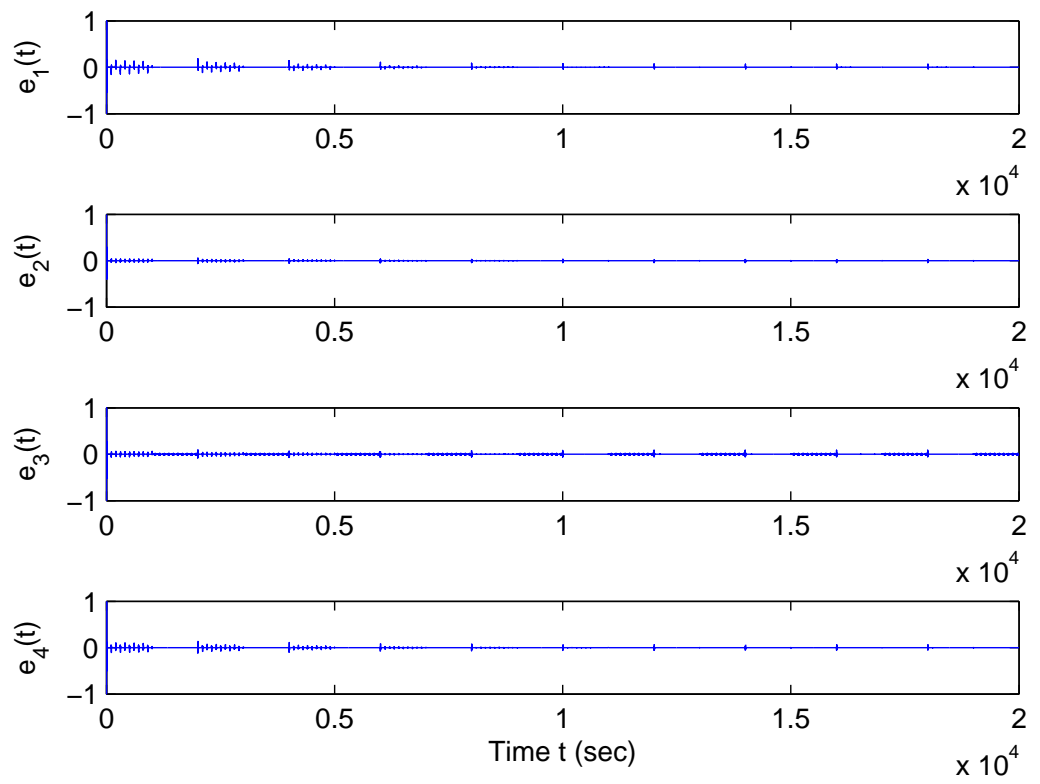

Figure 5.1: State tracking error $\boldsymbol{e}(t)$ for $r(t)=2 \sin (0.01 \pi t)$ and $T_{0}=1000 \mathrm{~s}$.

Case I: $r(t)=2 \sin (0.01 \pi t), T_{0}=1000 \mathrm{~s}$ (Fig. 5.1). It can be seen that the closedloop system is stable, but whenever a system model switch occurs, $\boldsymbol{e}(t)$ deviates from zero, and asymptotic tracking is not achieved.

Case II: $r(t)=2 \sin (0.01 \pi t)+2 \sin (0.02 \pi t)+2 \sin (0.005 \pi t), T_{0}=1000 s$ (Fig. 5.2). The state tracking error $\boldsymbol{e}(t)$ is plotted in Figure 5.2. With a sufficiently rich reference input signal and sufficiently large switching time interval, it can be seen that both closed-loop stability and asymptotic tracking performance are achieved.

\subsection{Design for Multiple-Input Systems}

In this section, we consider the multiple-input piecewise linear systems with dynamics offset (2.1.6), i.e.,

$$
\dot{\boldsymbol{x}}(t)=\boldsymbol{A}(t) \boldsymbol{x}(t)+\boldsymbol{B}(t) \boldsymbol{u}(t)+\boldsymbol{c}(t)
$$



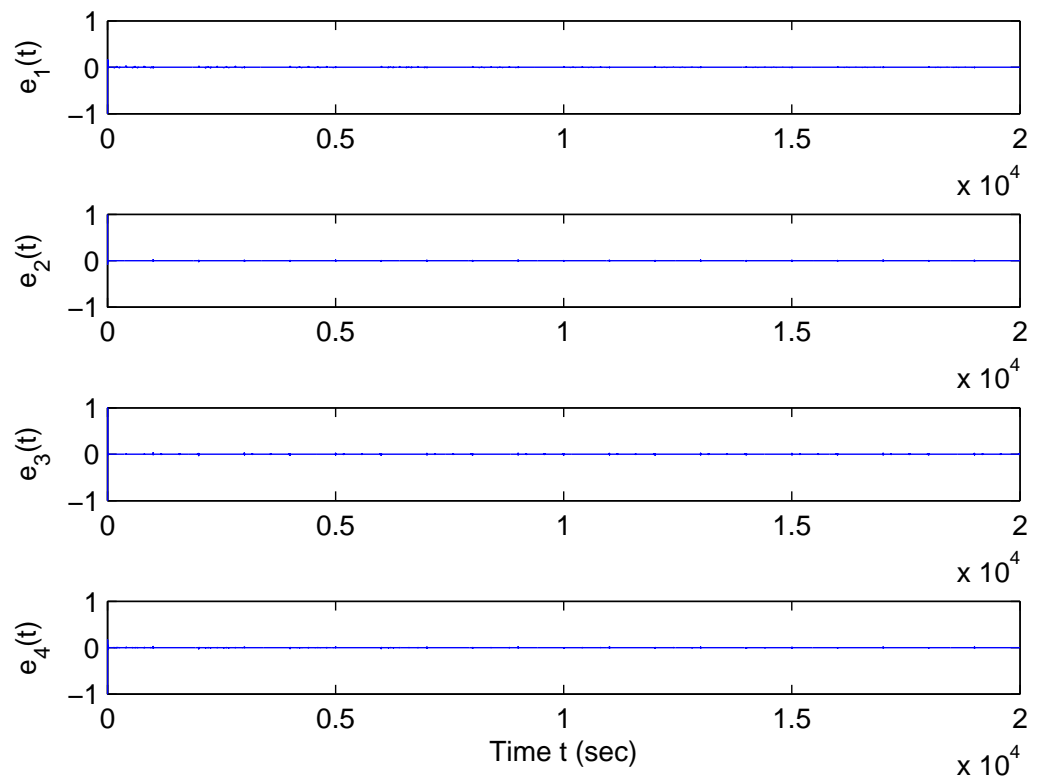

Figure 5.2: State tracking error $\boldsymbol{e}(t)$ for $r(t)=2 \sin (0.01 \pi t)+2 \sin (0.02 \pi t)+$ $2 \sin (0.005 \pi t)$ (sufficiently rich) and $T_{0}=1000 \mathrm{~s}$.

where $\boldsymbol{A}(t)=\sum_{i=1}^{l} \boldsymbol{A}_{i} \chi_{i}(t), \boldsymbol{B}(t)=\sum_{i=1}^{l} \boldsymbol{B}_{i} \chi_{i}(t), \boldsymbol{c}(t)=\sum_{i=1}^{l} \boldsymbol{c}_{i} \chi_{i}(t)$ with unknown $\boldsymbol{A}_{i}, \boldsymbol{B}_{i}, \boldsymbol{c}_{i}=-\boldsymbol{A}_{i} \boldsymbol{x}_{0 i}-\boldsymbol{B}_{i} \boldsymbol{u}_{0 i}$, and $\boldsymbol{x}(t), \boldsymbol{u}(t), \boldsymbol{x}_{0 i}, \boldsymbol{u}_{0 i}$ are defined as in Section 2.1.3. The triple $\left(\boldsymbol{A}_{i}, \boldsymbol{B}_{i}, \boldsymbol{c}_{i}\right)$ represents a subsystem of (5.4.1). For a basic study of the adaptive control problem for (5.4.1), it is assumed that there are no internally forced subsystem switches, i.e., $\chi_{i}(t)$ do not depend on $(\boldsymbol{x}(t), \boldsymbol{u}(t))$ :

$$
\chi_{i}(t)= \begin{cases}1, & \text { if }(\boldsymbol{A}(t), \boldsymbol{B}(t), \boldsymbol{c}(t))=\left(\boldsymbol{A}_{i}, \boldsymbol{B}_{i}, \boldsymbol{c}_{i}\right) \\ 0, & \text { otherwise }\end{cases}
$$

and satisfy (5.1.4).

The control objective is to develop a state feedback control law for the plant (5.4.1) such that all the signals in the closed-loop system are bounded, and $\boldsymbol{x}(t)$ asymptotically track a reference trajectory $\boldsymbol{x}_{m}(t)$, i.e., $\lim _{t \rightarrow \infty}\left(\boldsymbol{x}(t)-\boldsymbol{x}_{m}(t)\right)=\mathbf{0}$, where $\boldsymbol{x}_{m}(t)$ is generated from a reference model system. 


\subsubsection{Reference Model System}

A reference model for each subsystem is specified, resulting in a set of linear timeinvariant reference systems

$$
\dot{\boldsymbol{x}}_{m i}(t)=\boldsymbol{A}_{m i} \boldsymbol{x}_{m i}(t)+\boldsymbol{B}_{m i} \boldsymbol{r}(t)
$$

where $\boldsymbol{r}(t) \in \mathbb{R}^{m}$ is a bounded, piecewise continuous reference input signal, and the parameter matrices $\boldsymbol{A}_{m i} \in \mathbb{R}^{n \times n}, \boldsymbol{B}_{m i} \in \mathbb{R}^{n}, i \in \mathcal{I}$, are chosen with $\boldsymbol{A}_{m i}$ stable. When the $i$ th subsystem is active, the state trajectory $\boldsymbol{x}_{m i}(t)$ is desirable for the perturbed state $\boldsymbol{x}_{i}(t)$ to follow. To form a "global" reference trajectory for $\boldsymbol{x}(t)$ to track, with $\boldsymbol{x}_{m}(t) \triangleq \boldsymbol{x}_{m i}(t)+\boldsymbol{x}_{0 i}$ for $\chi_{i}(t)=1$, the piecewise linear reference model system for (5.4.1) is

$$
\dot{\boldsymbol{x}}_{m}(t)=\boldsymbol{A}_{m}(t) \boldsymbol{x}_{m}(t)+\boldsymbol{B}_{m}(t) \boldsymbol{r}(t)+\boldsymbol{c}_{m}(t)
$$

where $\boldsymbol{A}_{m}(t)=\sum_{i=1}^{l} \boldsymbol{A}_{m i} \chi_{i}(t), \boldsymbol{B}_{m}(t)=\sum_{i=1}^{l} \boldsymbol{B}_{m i} \chi_{i}(t)$, and $\boldsymbol{c}_{m}(t)=\sum_{i=1}^{l} \boldsymbol{c}_{m i} \chi_{i}(t)$ with $\boldsymbol{c}_{m i}=-\boldsymbol{A}_{m i} \boldsymbol{x}_{0 i}$. Note here $\boldsymbol{x}_{m}(t)$ is required to be continuous, which is a meaningful reference trajectory for the continuous state vector $\boldsymbol{x}(t)$ to follow. This implies a (perturbed) reference state resetting whenever a subsystem switch from the $i$ th to the $j$ th occurs at a time instant $t$ such that $\boldsymbol{x}_{m j}(t)+\boldsymbol{x}_{0 j}=\boldsymbol{x}_{m i}\left(t^{-}\right)+\boldsymbol{x}_{0 i}$.

Stability of the Reference Model System (5.4.2). The stability properties of the reference model system in (5.4.2) have been studied in $[1,19,39]$ without considering the dynamics offset term $\boldsymbol{c}_{m}(t)$. Following a similar line of arguments and derivations, it can be proved that the exponential stability of its homogeneous system implies stability of (5.4.2). In view of Lemma 5.2.1, this implies that the stability (signal boundedness) of (5.4.2) is ensured if the minimum switching time interval $T_{0}=\min _{k \in \mathbb{Z}^{+}}\left\{t_{k}-t_{k-1}\right\}$ between consecutive subsystem switches satisfies (5.2.4).

MRAC Assumptions. To meet the control objective, the following assumptions 
are made for $i \in \mathcal{I}$ :

(A5.4-1) There exist constant matrices $\boldsymbol{K}_{x i}^{*} \in \mathbb{R}^{n \times m}$ and $\boldsymbol{K}_{r i}^{*} \in \mathbb{R}^{m \times m}$ with $\boldsymbol{K}_{r i}^{*}$ nonsingular such that

$$
\boldsymbol{A}_{m i}=\boldsymbol{A}_{i}+\boldsymbol{B}_{i} \boldsymbol{K}_{x i}^{* \top}, \quad \boldsymbol{B}_{m i}=\boldsymbol{B}_{i} \boldsymbol{K}_{r i}^{*}
$$

(A5.4-2.a) There are known matrices $\boldsymbol{S}_{i} \in \mathbb{R}^{m \times m}$ such that $\boldsymbol{K}_{r i}^{*} \boldsymbol{S}_{i}$ are symmetric and positive definite.

\subsubsection{Controller Structure}

A new state feedback controller structure is proposed, and adaptive laws are developed for control of the piecewise linear plant (5.4.1) to achieve the control objective of closed-loop stability (signal boundedness) and state tracking.

If the plant parameter matrices $\boldsymbol{A}_{i}$ and $\boldsymbol{B}_{i}$ are known, the nominal control law

$$
\boldsymbol{u}(t)=\boldsymbol{u}_{0}(t)+\boldsymbol{K}_{x}^{* \boldsymbol{T}}(t) \Delta \boldsymbol{x}(t)+\boldsymbol{K}_{r}^{*}(t) \boldsymbol{r}(t)
$$

where $\boldsymbol{K}_{x}^{*}(t)=\sum_{i=1}^{l} \boldsymbol{K}_{x i}^{*} \chi_{i}(t), \boldsymbol{K}_{r}^{*}(t)=\sum_{i=1}^{l} \boldsymbol{K}_{r i}^{*} \chi_{i}(t), \Delta \boldsymbol{x}(t)=\boldsymbol{x}(t)-\boldsymbol{x}_{0}(t)$ with $\boldsymbol{x}_{0}(t)=\sum_{i=1}^{l} \boldsymbol{x}_{0 i} \chi_{i}(t)$ and $\boldsymbol{u}_{0}(t)=\sum_{i=1}^{l} \boldsymbol{u}_{0 i} \chi_{i}(t)$, leads to the tracking error dynamics $\dot{\boldsymbol{e}}(t)=\boldsymbol{A}_{m}(t) \boldsymbol{e}(t)$ with $\boldsymbol{e}(t)=\boldsymbol{x}(t)-\boldsymbol{x}_{m}(t)$ converging to zero exponentially if $T_{0}$ satisfies (5.2.4).

However, $\boldsymbol{A}_{i}$ and $\boldsymbol{B}_{i}$ are unknown in many practical applications, and (5.4.3) cannot be implemented. The following adaptive controller structure is applied

$$
\boldsymbol{u}(t)=\boldsymbol{u}_{0}(t)+\boldsymbol{K}_{x}^{\top}(t) \Delta \boldsymbol{x}(t)+\boldsymbol{K}_{r}(t) \boldsymbol{r}(t)
$$

where $\boldsymbol{K}_{x}(t)=\sum_{i=1}^{l} \boldsymbol{K}_{x i}(t) \chi_{i}(t), \boldsymbol{K}_{r}(t)=\sum_{i=1}^{l} \boldsymbol{K}_{r i}(t) \chi_{i}(t)$ are the time-varying estimates of the nominal controller parameters $\boldsymbol{K}_{x}^{*}(t)$ and $\boldsymbol{K}_{r}^{*}(t)$, respectively. This 
control law leads to the error model

$$
\dot{\boldsymbol{e}}=\sum_{i=1}^{l}\left(\boldsymbol{A}_{m i} \chi_{i} \boldsymbol{e}+\boldsymbol{B}_{m i} \boldsymbol{K}_{r i}^{*-1} \chi_{i}\left(\tilde{\boldsymbol{K}}_{x i}^{\top} \Delta \boldsymbol{x}+\tilde{\boldsymbol{K}}_{r i} \boldsymbol{r}\right)\right)
$$

with $\tilde{\boldsymbol{K}}_{x i}(t)=\boldsymbol{K}_{x i}(t)-\boldsymbol{K}_{x i}^{*}, \tilde{\boldsymbol{K}}_{r i}(t)=\boldsymbol{K}_{r i}(t)-\boldsymbol{K}_{r i}^{*}, i \in \mathcal{I}$

\subsubsection{Adaptive Laws}

Adaptive laws are developed based on the error model (5.4.5). The case when a common Lyapunov matrix exists for the constituent reference models $\boldsymbol{A}_{m i}$ is considered first.

\subsubsection{Adaptation when a common Lyapunov matrix $\boldsymbol{P}_{m}$ exists}

If for the stable reference model state matrices $\boldsymbol{A}_{m i}, i \in \mathcal{I}$, there exists a common Lyapunov matrix $\boldsymbol{P}_{m}=\boldsymbol{P}_{m}^{\top}>0$ such that

$$
\boldsymbol{A}_{m i}^{\top} \boldsymbol{P}_{m}+\boldsymbol{P}_{m} \boldsymbol{A}_{m i}<\mathbf{0}
$$

the following adaptive laws are proposed:

$$
\begin{aligned}
& \dot{\boldsymbol{K}}_{x i}^{\top}(t)=-\boldsymbol{S}_{i}^{\top} \boldsymbol{B}_{m i}^{\top} \chi_{i}(t) \boldsymbol{P}_{m} \boldsymbol{e}(t) \Delta \boldsymbol{x}^{\top}(t) \\
& \dot{\boldsymbol{K}}_{r i}(t)=-\boldsymbol{S}_{i}^{\top} \boldsymbol{B}_{m i}^{\top} \chi_{i}(t) \boldsymbol{P}_{m} \boldsymbol{e}(t) \boldsymbol{r}^{\top}(t)
\end{aligned}
$$

Such an adaptive control scheme has the following stability and tracking properties:

Theorem 5.4.1. If $\boldsymbol{A}_{m i}, i \in \mathcal{I}$, of the reference model system (5.4.2) satisfy (5.4.6) for some $\boldsymbol{P}_{m}=\boldsymbol{P}_{m}^{\top}>0$, then all signals in the closed-loop system are bounded, and the state tracking error $\boldsymbol{e}(t)=\boldsymbol{x}(t)-\boldsymbol{x}_{m}(t)$ converges to zero asymptotically, for arbitrary subsystem switches.

$\underline{\text { Proof: }}$ Let $\boldsymbol{A}_{m i}^{\top} \boldsymbol{P}_{m}+\boldsymbol{P}_{m} \boldsymbol{A}_{m i}=-\boldsymbol{Q}_{m i}$ for some $\boldsymbol{Q}_{m i}=\boldsymbol{Q}_{m i}^{\top}>\mathbf{0}, i \in \mathcal{I}$. Consider the 
Lyapunov function candidate

$$
\begin{aligned}
V & =\boldsymbol{e}^{\top} \boldsymbol{P} \boldsymbol{e}+\sum_{i=1}^{l}\left(\sum_{j=1}^{n} \tilde{\boldsymbol{k}}_{x i j}^{\top} \boldsymbol{M}_{s i}^{-1} \tilde{\boldsymbol{k}}_{x i j}+\sum_{j=1}^{m} \tilde{\boldsymbol{k}}_{r i j}^{\top} \boldsymbol{M}_{s i}^{-1} \tilde{\boldsymbol{k}}_{r i j}\right) \\
& =\boldsymbol{e}^{\top} \boldsymbol{P} \boldsymbol{e}+\sum_{i=1}^{l}\left(\operatorname{tr}\left[\tilde{\boldsymbol{K}}_{x i} \boldsymbol{M}_{s i}^{-1} \tilde{\boldsymbol{K}}_{x i}^{\top}\right]+\operatorname{tr}\left[\tilde{\boldsymbol{K}}_{r i}^{\top} \boldsymbol{M}_{s i}^{-1} \tilde{\boldsymbol{K}}_{r i}\right]\right)
\end{aligned}
$$

where $\boldsymbol{M}_{s i}=\boldsymbol{K}_{r i}^{*} \boldsymbol{S}_{i}, \tilde{\boldsymbol{K}}_{x i}^{\top}(t)=\left[\tilde{\boldsymbol{k}}_{x i 1}, \tilde{\boldsymbol{k}}_{x i 2} \ldots, \tilde{\boldsymbol{k}}_{x i n}\right], \tilde{\boldsymbol{K}}_{r i}(t)=\left[\tilde{\boldsymbol{k}}_{r i 1}, \tilde{\boldsymbol{k}}_{r i 2}, \ldots, \tilde{\boldsymbol{k}}_{r i m}\right]$, and $\operatorname{tr}[\cdot]$ denotes the trace of a square matrix. Under Assumption (A5.4-2.a) and with the facts that $\operatorname{tr}\left[\boldsymbol{M}_{1} \boldsymbol{M}_{2}\right]=\operatorname{tr}\left[\boldsymbol{M}_{2} \boldsymbol{M}_{1}\right], \operatorname{tr}\left[\boldsymbol{M}_{3}\right]=\operatorname{tr}\left[\boldsymbol{M}_{3}^{\top}\right]$ for any matrices $\boldsymbol{M}_{i}, i=1,2,3$, of compatible dimensions, its time derivative along (5.4.7) is

$$
\dot{V} \leq-\left(\min _{i \in \mathcal{I}} \lambda_{\min }\left[\boldsymbol{Q}_{m i}\right]\right)\|\boldsymbol{e}\|^{2}
$$

It follows that $\boldsymbol{e}(t) \in \mathcal{L}^{2} \cap \mathcal{L}^{\infty}, \boldsymbol{K}_{x i}(t), \boldsymbol{K}_{r i}(t) \in \mathcal{L}^{\infty}$, and with $\boldsymbol{x}_{m}(t) \in \mathcal{L}^{\infty}, \boldsymbol{u}(t), \dot{\boldsymbol{e}}(t) \in$ $\mathcal{L}^{\infty}$. Therefore, all signals in the closed-loop system are bounded, and according to Barbălat Lemma [15], $\lim _{t \rightarrow \infty} \boldsymbol{e}(t)=\mathbf{0}$.

Only a set of matrices in certain special structures is known to have a common $\boldsymbol{P}_{m}$ matrix, hence such a design cannot be extended to a general set of reference system matrices $\boldsymbol{A}_{m i}$. Next, adaptive laws are presented and studied for the case when no such common $\boldsymbol{P}_{m}$ exists.

\subsubsection{Adaptation when a common $\boldsymbol{P}_{m}$ does not exist}

When no common Lyapunov matrix $\boldsymbol{P}_{m}$ satisfying (5.4.6) exists for the set of stable matrices $\boldsymbol{A}_{m i}, i \in \mathcal{I}$, the parameter projection adaptive laws are applied, with the assumption of certain knowledge of lower and upper bounds on the controller parameters, as follows:

$$
\begin{aligned}
& \dot{\boldsymbol{K}}_{x i}^{\top}(t)=-\boldsymbol{S}_{i}^{\top} \boldsymbol{B}_{m i}^{\top} \chi_{i}(t) \boldsymbol{P}_{m i} \boldsymbol{e}(t) \Delta \boldsymbol{x}^{\top}(t)+\boldsymbol{F}_{x i}(t) \\
& \dot{\boldsymbol{K}}_{r i}(t)=-\boldsymbol{S}_{i}^{\top} \boldsymbol{B}_{m i}^{\top} \chi_{i}(t) \boldsymbol{P}_{m i} \boldsymbol{e}(t) \boldsymbol{r}^{\top}(t)+\boldsymbol{F}_{r i}(t)
\end{aligned}
$$


to update the controller parameters in (5.4.4), where $\boldsymbol{P}_{m i}=\boldsymbol{P}_{m i}^{\top}>\mathbf{0}, i \in \mathcal{I}$, satisfy the Lyapunov equations

$$
\boldsymbol{A}_{m i}^{\top} \boldsymbol{P}_{m i}+\boldsymbol{P}_{m i} \boldsymbol{A}_{m i}=-\boldsymbol{Q}_{m i}, \quad \boldsymbol{Q}_{m i}=\boldsymbol{Q}_{m i}^{\top}>\mathbf{0}
$$

For such an adaptive control design with parameter projection to be effective, a further assumption based on Assumption (A5.4-2.a) is made:

(A5.4-2.b) The known matrix $\boldsymbol{S}_{i}$ in Assumption (A5.4-2.a) is such that $\boldsymbol{K}_{r i}^{*} \boldsymbol{S}_{i}$ is diagonal and positive definite.

The initial estimate of each element in $\boldsymbol{K}_{x i}(t), \boldsymbol{K}_{r i}(t)$ is chosen to be within its known bounds. The projection terms $\boldsymbol{F}_{x i}(t), \boldsymbol{F}_{r i}(t)$ are so defined as to confine the parameter estimates inside these bounds for all time: parameter adaptation is active (i.e., $\boldsymbol{F}_{x i}(t)=\mathbf{0}, \boldsymbol{F}_{r i}(t)=\mathbf{0}$ ) when the estimates are within those bounds, while it is deactivated otherwise, and the estimates are left unchanged (i.e., $\dot{\boldsymbol{K}}_{x i}(t)=\mathbf{0}$, $\left.\dot{\boldsymbol{K}}_{r i}(t)=\mathbf{0}\right)$.

With the definitions of $a_{m}, \lambda_{m}, \alpha, \beta, \mu, \Delta_{\boldsymbol{A}_{m}}$ in Lemma 5.2.1, the following stability and tracking properties follow:

Theorem 5.4.2. Consider the closed-loop system with the plant (5.4.1), the reference model (5.4.2), and the controller (5.4.4) updated by the adaptive laws (5.4.9). If

$$
T_{0} \geq T_{d}=\alpha(1+\kappa) \ln \left(1+\mu \Delta_{A_{m}}\right), \quad \kappa>0
$$

then all closed-loop signals are bounded, and the tracking error $\boldsymbol{e}(t)$ is small in the sense that

$$
\int_{t}^{t+T} \boldsymbol{e}^{\top}(\tau) \boldsymbol{e}(\tau) d \tau \leq \mu \Delta_{\boldsymbol{A}_{m}} c_{0} \frac{T}{T_{0}}+c_{1}, \quad \forall t \geq t_{0}, \quad \forall T>0
$$

with $c_{1}=\left(1+\mu \Delta_{\boldsymbol{A}_{m}}\right) c_{0}$, for some $c_{0}>0$. 
Proof: The proof follows the same line as the proof of Theorem 5.3.1 by considering the piecewise continuous Lyapunov function

$$
V=\boldsymbol{e}^{\top} \sum_{i=1}^{l} \boldsymbol{P}_{m i} \chi_{i} \boldsymbol{e}+\sum_{i=1}^{l}\left(\sum_{j=1}^{n} \tilde{\boldsymbol{k}}_{x i j}^{\top} \boldsymbol{M}_{s i}^{-1} \tilde{\boldsymbol{k}}_{x i j}+\sum_{j=1}^{m} \tilde{\boldsymbol{k}}_{r i j}^{\top} \boldsymbol{M}_{s i}^{-1} \tilde{\boldsymbol{k}}_{r i j}\right)
$$

with $\boldsymbol{M}_{s i}=\boldsymbol{K}_{r i}^{*} \boldsymbol{S}_{i}=\operatorname{diag}\left[m_{s i 1}, \ldots, m_{s i m}\right]>\mathbf{0}, \tilde{\boldsymbol{K}}_{x i}^{\top}=\left[\tilde{\boldsymbol{k}}_{x i 1}, \ldots, \tilde{\boldsymbol{k}}_{x i n}\right], \tilde{\boldsymbol{K}}_{r i}=$ $\left[\tilde{\boldsymbol{k}}_{r i 1}, \ldots, \tilde{\boldsymbol{k}}_{r i m}\right]$, and the details are omitted.

\subsubsection{Adaptation with sufficiently rich reference input $r(t)$}

If some of the subsystems with indices $i \in \mathcal{I}^{*} \subset \mathcal{I}$ are no longer active after a finite time $T_{i} \geq t_{0}$, while other subsystems are active intermittently over infinitely many intervals, then under the persistency of excitation condition, the following stability and tracking properties for $t \geq T^{*}=\max _{i \in \mathcal{I}^{*}}\left\{T_{i}\right\}$ follow:

Theorem 5.4.3. Consider the closed-loop system with the plant (5.4.1), the reference model (5.4.2), and the controller (5.4.4) updated by the adaptive laws

$$
\begin{aligned}
& \dot{\boldsymbol{K}}_{x i}^{\top}(t)=-\boldsymbol{S}_{i}^{\top} \boldsymbol{B}_{m i}^{\top} \chi_{i}(t) \boldsymbol{P}_{m i} \boldsymbol{e}(t) \Delta \boldsymbol{x}^{\top}(t) \\
& \dot{\boldsymbol{K}}_{r i}(t)=-\boldsymbol{S}_{i}^{\top} \boldsymbol{B}_{m i}^{\top} \chi_{i}(t) \boldsymbol{P}_{m i} \boldsymbol{e}(t) \boldsymbol{r}^{\top}(t)
\end{aligned}
$$

Suppose each element $r_{i}(t), i=1,2, \ldots, m$, of the reference input $\boldsymbol{r}(t)$ is sufficiently rich of order $n+1$ and uncorrelated, and $\left(\boldsymbol{A}_{m i}, \boldsymbol{B}_{m i}\right), i \in \mathcal{I}$, are controllable. If the switching time intervals are sufficiently large, then all closed-loop signals are bounded for $t \geq t_{0} ; \boldsymbol{e}(t), \tilde{\boldsymbol{K}}_{x i}(t), \tilde{\boldsymbol{K}}_{r i}(t)$ converge to zero exponentially, $i \in \mathcal{I}-\mathcal{I}^{*}, t \geq T^{*}$; and $\boldsymbol{K}_{x i}(t)=\boldsymbol{K}_{x i}\left(T_{i}\right), \boldsymbol{K}_{r i}(t)=\boldsymbol{K}_{r i}\left(T_{i}\right), i \in \mathcal{I}^{*}, t \geq T_{i}$.

Proof: The proof follows the same line as the proof of Theorem 5.3.5, and is thus omitted. 


\subsubsection{Simulation on NASA GTM}

Simulations are performed to demonstrate the system stability and tracking performance with the proposed adaptive control schemes applied to the piecewise linear system model of the longitudinal dynamics of the NASA GTM at multiple operating points, as well as the nonlinear GTM [82].

\section{Linearized Aircraft Longitudinal Model and Reference Model System.}

An operating point for a nonlinear aircraft system is specified by $(V, h)$, with $V$ and $h$ denoting the vehicle speed and altitude, respectively. At a specific operating point $\left(V_{i}, h_{i}\right)$, a trim point (equilibrium) $\left(\boldsymbol{x}_{0 i}, \boldsymbol{u}_{0 i}\right)$ may be found, where $\boldsymbol{x}_{0 i}$ is the nominal state vector, and $\boldsymbol{u}_{0 i}$ is the nominal input vector to the system. In steady-state, straight, level flight, the longitudinal and lateral-directional dynamics of an aircraft are decoupled from each other, and the linearized longitudinal model of an aircraft around $\left(\boldsymbol{x}_{0 i}, \boldsymbol{u}_{0 i}\right)$ can be represented by (2.1.5), i.e.,

$$
\dot{\boldsymbol{x}}_{i}=\boldsymbol{A}_{i} \boldsymbol{x}_{i}+\boldsymbol{B}_{i} \boldsymbol{u}_{i}, \quad \boldsymbol{x}_{i}=[u, w, q, \theta]^{\top}, \quad \boldsymbol{u}_{i}=\left[\delta_{e}, \delta_{T}\right]^{\top}
$$

where $u, w, q, \theta$ are the perturbed aircraft velocity components along the $\mathrm{x}$ - and z-body-axis (fps), angular velocity along the y-body-axis (crad/s), and pitch angle $(\mathrm{crad})$, respectively; that is, $\boldsymbol{x}_{i}(t)=\boldsymbol{x}(t)-\boldsymbol{x}_{0 i}$ with $\boldsymbol{x}(t)$ being the aircraft longitudinal state vector. The control input vector $\boldsymbol{u}_{i}(t)$ consists of the perturbed elevator deflection $\delta_{e}$ and throttle input $\delta_{T}$, i.e., $\boldsymbol{u}_{i}(t)=\boldsymbol{u}(t)-\boldsymbol{u}_{0 i}$ with $\boldsymbol{u}(t)$ being the total control applied to the aircraft. In terms of the original state and control vector $\boldsymbol{x}(t)$ and $\boldsymbol{u}(t)$, the linearized longitudinal model is $\dot{\boldsymbol{x}}(t)=\boldsymbol{A}_{i} \boldsymbol{x}(t)+\boldsymbol{B}_{i} \boldsymbol{u}(t)+\boldsymbol{c}_{i}$ with $\boldsymbol{c}_{i}=-\boldsymbol{A}_{i} \boldsymbol{x}_{0 i}-\boldsymbol{B}_{i} \boldsymbol{u}_{0 i}$.

The desired longitudinal behavior of the aircraft within a neighborhood of $\left(\boldsymbol{x}_{0 i}, \boldsymbol{u}_{0 i}\right)$ is specified by a reference model in the form $\dot{\boldsymbol{x}}_{m i}=\boldsymbol{A}_{m i} \boldsymbol{x}_{m i}+\boldsymbol{B}_{m i} \boldsymbol{r}$, where $\boldsymbol{r}(t)$ is 
the reference input vector that can generate the desired (perturbed) state trajectory $\boldsymbol{x}_{m i}(t)$. Here $\boldsymbol{x}_{m i}(t)$ is defined with regard to $\boldsymbol{x}_{0 i}$. In terms of a "global" reference trajectory $\boldsymbol{x}_{m}(t)=\boldsymbol{x}_{m i}(t)+\boldsymbol{x}_{0 i}$, with $\boldsymbol{c}_{m i}=-\boldsymbol{A}_{m i} \boldsymbol{x}_{0 i}$, the reference model system is obtained as follows:

$$
\dot{\boldsymbol{x}}_{m}(t)=\boldsymbol{A}_{m}(t) \boldsymbol{x}_{m}(t)+\boldsymbol{B}_{m}(t) \boldsymbol{r}(t)+\boldsymbol{c}_{m}(t)
$$

In this simulation study, LQ controllers in the form $\boldsymbol{u}_{\mathrm{nom}}(t)=\boldsymbol{u}_{0 i}(t)+\boldsymbol{K}_{x i}^{* \boldsymbol{\top}} \Delta \boldsymbol{x}(t)+\boldsymbol{r}(t)$ are designed based on $\left(\boldsymbol{A}_{i}, \boldsymbol{B}_{i}\right)$ (not used in adaptive control design), and the reference model systems are chosen such that Assumption (A5.4-1) is satisfied with $\boldsymbol{K}_{r i}^{*}=\boldsymbol{I}_{n}$. In other words, the nominal linearized closed-loop system dynamics are chosen as the reference model system. Note that with such a choice, Assumptions (A5.4-2.a) and (A5.4-2.b) are satisfied as well. In particular, $\boldsymbol{S}_{i}$ can be any positive definite diagonal matrix.

Switches of Operating Points. Extensive simulations are performed to determine the valid linearization regions around the trim points and decent switching surfaces in between. In particular, for each trim point $\left(\boldsymbol{x}_{0 i}, \boldsymbol{u}_{0 i}\right)$, a reference input vector signal $\boldsymbol{r}(t)$ relatively small in magnitude is chosen such that the GTM longitudinal states $\boldsymbol{x}(t)$ stay within $\boldsymbol{\Omega}_{i}$. A $\boldsymbol{r}(t)$ with relatively large magnitude is also determined which can drive $\boldsymbol{x}(t)$ to cross the switching surface, corresponding a desired change of operating point.

Remark 5.4.4. In the piecewise linear system framework, the closed-loop stability and tracking performance are guaranteed for arbitrary $\boldsymbol{S}_{i}$ satisfying Assumption (A5.42.b). The diagonal, positive definite matrices $\boldsymbol{S}_{i}$ are in essence the adaptation gain matrices that can be adjusted for desirable adaptation rate. In general, large $\boldsymbol{S}_{i}$ leads to fast adaptation and better performance. However, this may not be true when such a 
linearization-based design is applied to the nonlinear GTM, in which fast adaptation may destabilize the system due to high frequency transients and unmodeled system dynamics. In this simulation study, $\boldsymbol{S}_{i}$ is chosen based on the system performance observed in the simulations.

Design and Simulation Parameters. For simplicity of presentation, the number of subsystems is chosen to be $l=2$, and the GTM is trimmed at steady-state, straight, wings-level flight condition at 75 knots and 85 knots at $800 \mathrm{ft}$., respectively, to obtain a piecewise linear longitudinal system model; to be specific,

$$
\begin{aligned}
& \boldsymbol{x}_{01}=[127.6597,10.3615,0,8.0987]^{\top}, \quad \boldsymbol{u}_{01}=[1.3109,14.1457]^{\top} \\
& \boldsymbol{x}_{02}=[144.9022,8.5931,0,5.9234]^{\top}, \quad \boldsymbol{u}_{02}=[2.3649,14.8592]^{\top} \\
& \begin{aligned}
\boldsymbol{A}_{1}= & {\left[\begin{array}{rrrr}
-0.0190 & 0.0825 & -0.1005 & -0.3206 \\
-0.2154 & -2.7859 & 1.2031 & -0.0271 \\
3.2527 & -30.7871 & -3.5418 & 0 \\
0 & 0 & 1.0000 & 0
\end{array}\right] } \\
\boldsymbol{B}_{1}= & {\left[\begin{array}{rr}
0.0065 & 0.0534 \\
-0.6103 & 0.0020 \\
-74.6355 & 0.5431 \\
0 & 0
\end{array}\right], \quad \boldsymbol{c}_{1}=\left[\begin{array}{r}
3.4011 \\
57.3601 \\
-6.0836 \\
0
\end{array}\right] }
\end{aligned} \\
& \boldsymbol{A}_{2}=\left[\begin{array}{rrrr}
-0.0312 & 0.1095 & -0.0938 & -0.3210 \\
-0.1057 & -3.2245 & 1.3765 & -0.0217 \\
3.9602 & -33.8308 & -4.0756 & 0 \\
0 & 0 & 1.0000 & 0
\end{array}\right] \\
& \boldsymbol{B}_{2}=\left[\begin{array}{rr}
0.0032 & 0.0534 \\
-0.7821 & 0.0020 \\
-96.0149 & 0.5431 \\
0 & 0
\end{array}\right], \quad \boldsymbol{c}_{2}=\left[\begin{array}{r}
4.6742 \\
44.9782 \\
-64.1394 \\
0
\end{array}\right]
\end{aligned}
$$

The reference model system is specified by LQ designs with $\boldsymbol{Q}=\boldsymbol{I}_{4}, \boldsymbol{R}=10 \boldsymbol{I}_{2}$; in 
particular, $\boldsymbol{B}_{m 1}=\boldsymbol{B}_{1}, \boldsymbol{B}_{m 2}=\boldsymbol{B}_{2}$,

$$
\begin{aligned}
\boldsymbol{A}_{m 1} & =\left[\begin{array}{rrrr}
-0.0215 & 0.0810 & -0.0988 & -0.3180 \\
-0.0706 & -2.6377 & 1.0345 & -0.2636 \\
20.9585 & -12.6579 & -24.1637 & -28.9269 \\
0 & 0 & 1.0000 & 0
\end{array}\right] \\
\boldsymbol{A}_{m 2} & =\left[\begin{array}{rrrr}
-0.0328 & 0.1088 & -0.0930 & -0.3196 \\
0.0753 & -3.0601 & 1.1577 & -0.3276 \\
26.1845 & -13.6389 & -30.9393 & -37.5452 \\
0 & 0 & 1.0000 & 0
\end{array}\right]
\end{aligned}
$$

thus $\boldsymbol{K}_{r 1}^{*}=\boldsymbol{K}_{r 2}^{*}=\boldsymbol{I}_{2}$, and

$$
\begin{aligned}
\boldsymbol{K}_{x 1}^{*} & =\left[\begin{array}{rrrr}
-0.2374 & -0.2429 & 0.2763 & 0.3876 \\
-0.0178 & 0.0009 & -0.0018 & 0.0011
\end{array}\right]^{\top} \\
\boldsymbol{K}_{x 2}^{*} & =\left[\begin{array}{rrrr}
-0.2316 & -0.2103 & 0.2798 & 0.3910 \\
-0.0173 & 0.0002 & -0.0015 & 0.0020
\end{array}\right]^{\top}
\end{aligned}
$$

It is found that a common Lyapunov matrix exists such that (5.4.6) is satisfied, thus the adaptive design in Section 5.4.3.1 may be applied. The matrices $\boldsymbol{S}_{i}$ are chosen based on the observed closed-loop GTM system performance. Here, $\boldsymbol{S}_{1}=0.05 \boldsymbol{I}_{4}$ and $\boldsymbol{S}_{2}=0.05 \boldsymbol{I}_{4}$. The reference input signal $\boldsymbol{r}(t)$ is selected as $\boldsymbol{r}(t)=[2 \sin (0.02 \pi t), 0]^{\top}$ to specify a longitudinal reference state trajectory for the GTM at each operating point; in the nonlinear simulations, it is set to be $\boldsymbol{r}(t)=\left[\begin{array}{ll}5, & 0\end{array}\right]^{\top}$ whenever there is a desired transition from the first operating point ( 75 knots, $800 \mathrm{ft}$.) to the second (85 knots, $800 \mathrm{ft}$. ), and $\boldsymbol{r}(t)=[-5,0]^{\top}$, otherwise. Since the parameter matrices of the linearized longitudinal model of the GTM are not sensitive to altitude variations within a relatively small range ( $\pm 100 \mathrm{ft}$.), as can be verified by linearizing the GTM at the same airspeed but different altitudes, the switching plane is specified by $V$ only. In this simulation study, $V$ is chosen to be $V=80$ knots.

For all the simulations, the GTM is initially trimmed at 75 knots, $800 \mathrm{ft}$., steadystate, straight, wings-level flight. A switch of operating point is commanded through the setting of $\boldsymbol{r}(t)$ every 100s. The initial tracking error is $\boldsymbol{e}(0)=\left[\begin{array}{ll}7,-4, & 0,50\end{array}\right]^{\top}$, and the initial parameter estimates are set as $60 \%$ of their nominal values. 
Simulation Results. Figure 5.3 shows the state tracking error $\boldsymbol{e}(t)$ for the adaptive control scheme in Section 5.4.3.1 applied to the piecewise linear model of the GTM. A convergence of the state tracking error $\boldsymbol{e}(t)$ to zero is observed. The tracking performance with the fixed control scheme in the form of (5.4.3) with controller parameters to be $60 \%$ of their nominal values is shown in 5.3 (bottom). It can be seen that whenever there is a subsystem switch, the tracking error deviates from zero.

To show the effectiveness of the proposed adaptive control scheme on system performance improvement, the nonlinear GTM system response is shown next, along with one obtained by applying the $60 \%$ fixed control scheme. Figure 5.4 shows the state tracking error $\boldsymbol{e}(t)$ when the adaptive control designs in Section 5.4.3.1 (above) and the $60 \%$ fixed control (bottom), respectively, are applied to the nonlinear GTM. All other system signals are bounded. The corresponding control input signals are shown in Fig. 5.5.

From the simulation results, it can be seen that the closed-loop stability is achieved for all the simulations. As for the state tracking, the proposed adaptive control schemes provide substantially improved performance over the fixed control law under the same flight conditions. The simulation results demonstrate the effectiveness of the proposed linearization-based adaptive control designs applied to the nonlinear GTM system.

\subsection{Extensions to State-Dependent Switches}

In this section, the adaptive state feedback control problem is considered for a singleinput, bimodel piecewise linear system with dynamic offsets and state-dependent subsystem switches. The desired state trajectory for the plant state to track is generated 

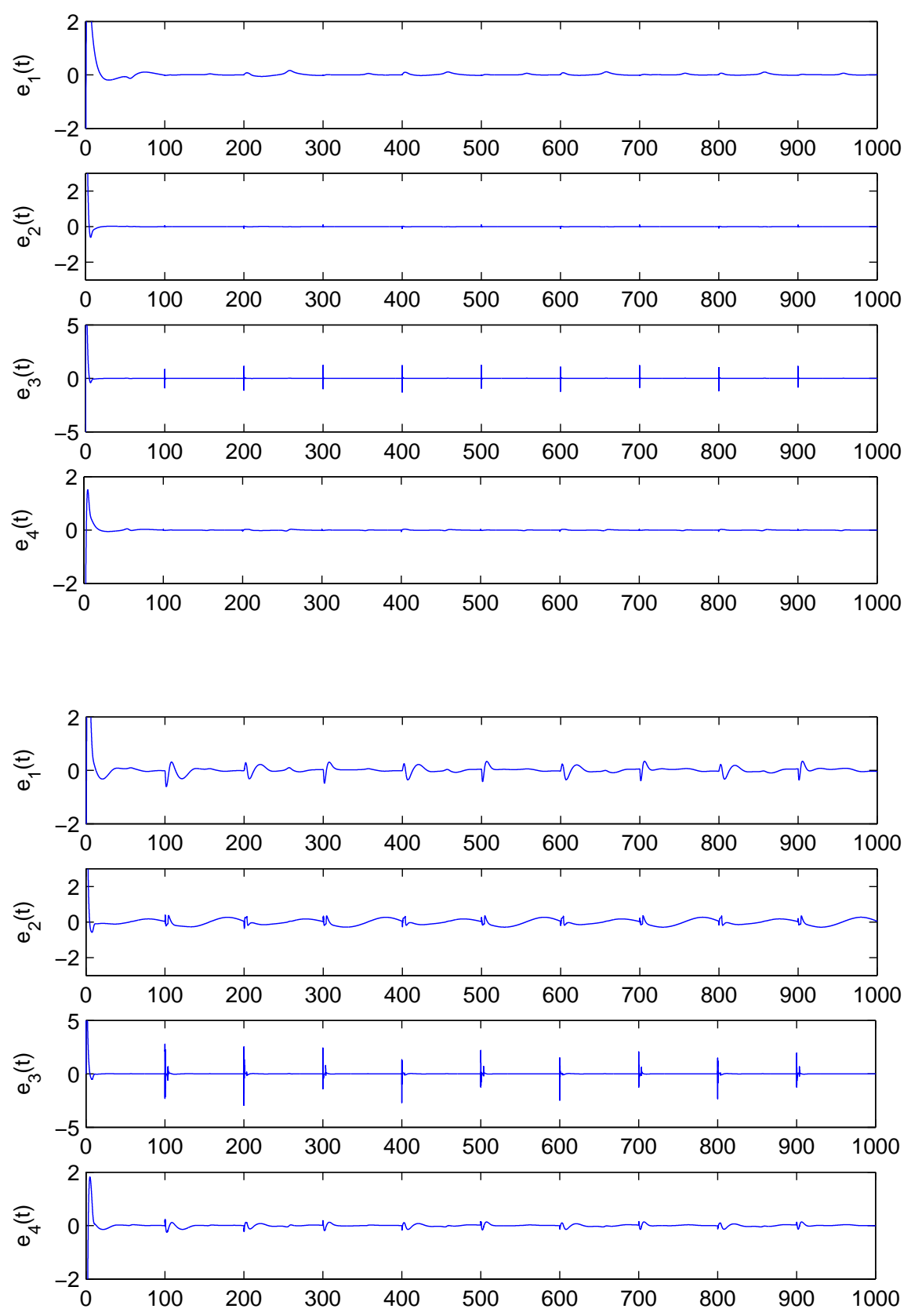

Figure 5.3: Simulations for linear system: state tracking error $\boldsymbol{e}(t)$ for the proposed control scheme (above) and for the 60\% fixed control law (below). 

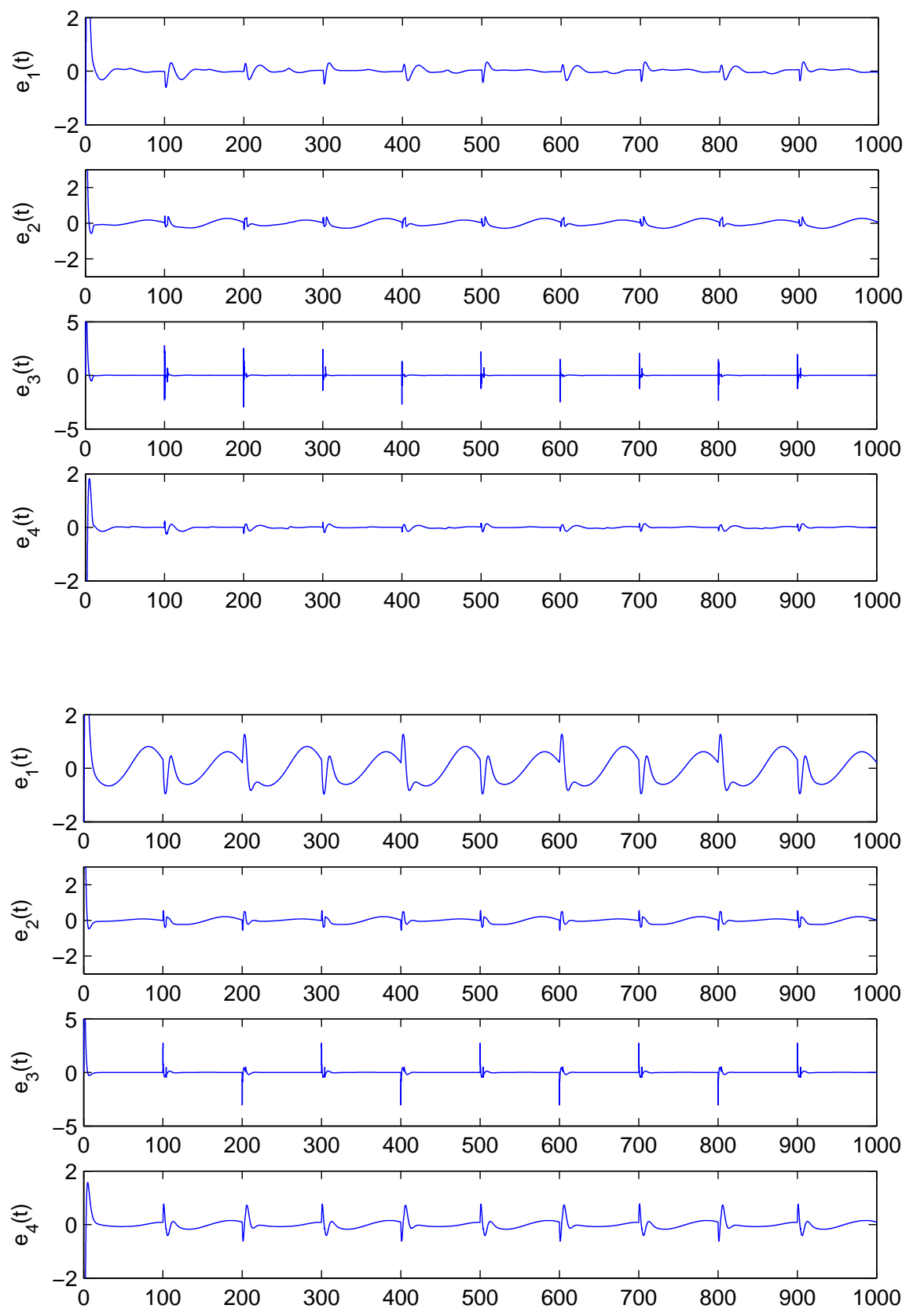

Figure 5.4: GTM simulation: $\boldsymbol{e}(t)$ for the proposed control scheme in Section 5.4.3.1 (above) and for the $60 \%$ fixed control law (below). 

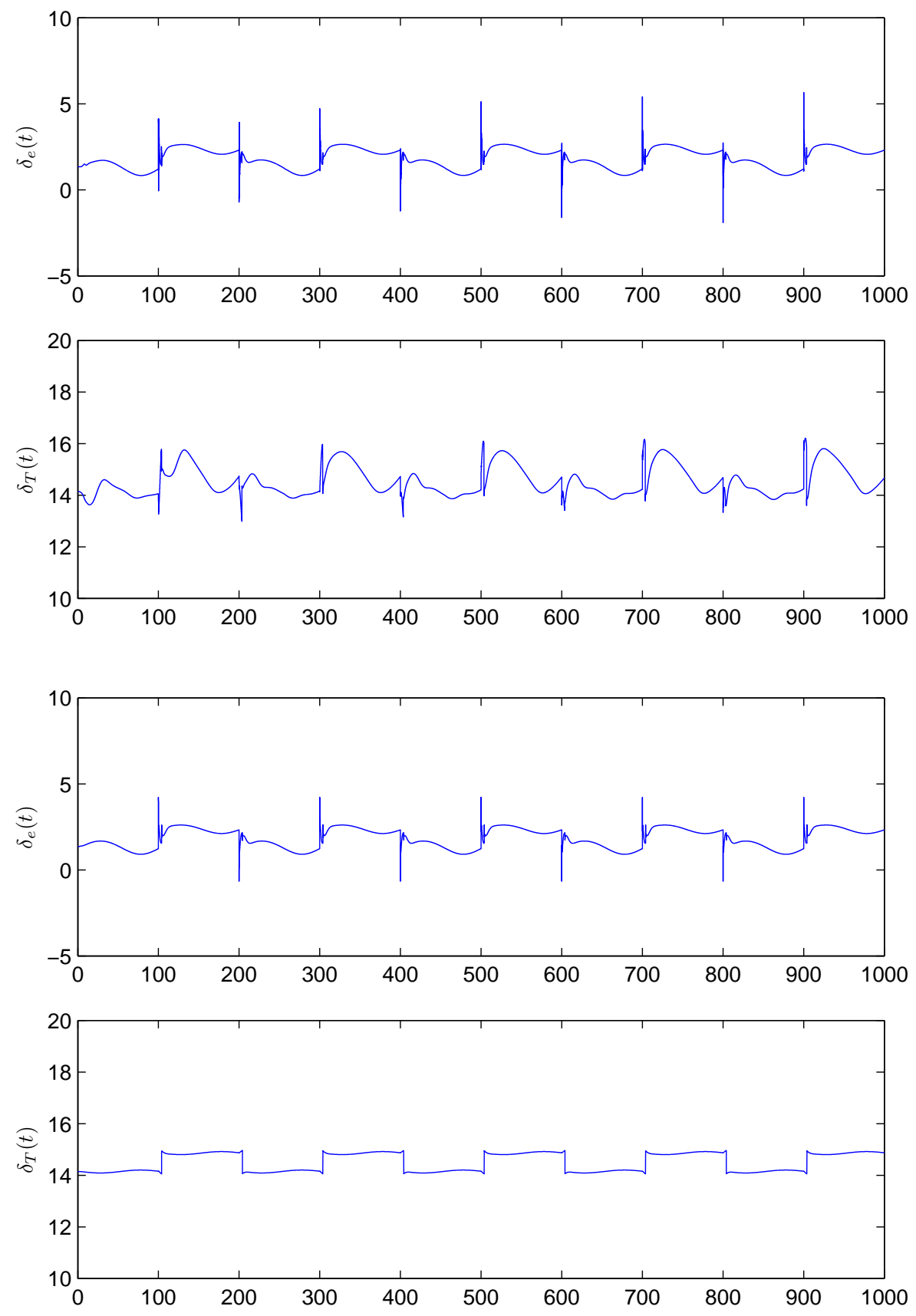

Figure 5.5: GTM simulation: control input $\boldsymbol{u}(t)=\left[\delta_{e}, \delta_{T}\right]^{\top}$ for the control scheme in Section 5.4.3.1 (above) and for the 60\% fixed control law (below). 
from a reference model system in a similar form. When the state matrices of each subsystem of the reference model system share a common Lyapunov matrix, closedloop signal boundedness and asymptotic state tracking performance can be achieved under certain plant-model matching conditions. Although only the bimodal case is considered here, straightforward modifications can be made such that the scheme becomes applicable to systems with more than two subsystems to achieve the desired stability properties and tracking performance.

\subsubsection{Problem Statement}

Consider a bimodel piecewise linear system described by:

$$
\dot{\boldsymbol{x}}=\boldsymbol{A}_{i} \boldsymbol{x}+\boldsymbol{b}_{i} u+\boldsymbol{b}_{i}^{0}, \quad \boldsymbol{x} \in \Omega_{i}, \quad i \in\{1,2\}
$$

where $\boldsymbol{x}(t) \in \mathbb{R}^{n}$ is the state vector, $u(t) \in \mathbb{R}$ is the control input, and $\boldsymbol{A}_{i}, \boldsymbol{b}_{i}, \boldsymbol{b}_{i}^{0}, i=$ 1,2 , are unknown constant parameter matrices (vectors) of appropriate dimensions. The system state space is partitioned into two polyhedral cells defined as follows:

$$
\begin{aligned}
& \Omega_{1}=\left\{\boldsymbol{x} \in \mathbb{R}^{n} \mid \boldsymbol{H}^{\top} \boldsymbol{x}+h \leq 0\right\} \\
& \Omega_{2}=\left\{\boldsymbol{x} \in \mathbb{R}^{n} \mid \boldsymbol{H}^{\top} \boldsymbol{x}+h>0\right\}
\end{aligned}
$$

It is assumed that the hyperplane, $\boldsymbol{H}^{\top} \boldsymbol{x}+h=0$, defining the polyhedral cells is known; that is, $\boldsymbol{H} \in \mathbb{R}^{n}, h \in \mathbb{R}$ are known.

The reference model system to generate the desired state trajectory $\boldsymbol{x}_{m}(t)$ for the system state $\boldsymbol{x}(t)$ to track is

$$
\dot{\boldsymbol{x}}_{m}=\boldsymbol{A}_{m i} \boldsymbol{x}_{m}+\boldsymbol{b}_{m i} r+\boldsymbol{b}_{m i}^{0}, \quad \boldsymbol{x}_{m} \in \Omega_{m i}, \quad i \in\{1,2\}
$$

where $\boldsymbol{A}_{m i}$ is stable, and $r(t)$ is a bounded, piecewise continuous reference input signal. The reference model state space is partitioned by a known hyperplane, $\boldsymbol{H}_{m}^{\top} \boldsymbol{x}+h_{m}=0$, 
into two polyhedral cells defined by

$$
\begin{aligned}
& \Omega_{m 1}=\left\{\boldsymbol{x}_{m} \in \mathbb{R}^{n} \mid \boldsymbol{H}_{m}^{\top} \boldsymbol{x}_{m}+h_{m} \leq 0\right\} \\
& \Omega_{m 2}=\left\{\boldsymbol{x}_{m} \in \mathbb{R}^{n} \mid \boldsymbol{H}_{m}^{\top} \boldsymbol{x}_{m}+h_{m}>0\right\}
\end{aligned}
$$

Note that $\boldsymbol{H}_{m}^{\top} \boldsymbol{x}+h_{m}=0$ may not be the same as $\boldsymbol{H}^{\top} \boldsymbol{x}+h=0$; that is, the subsystem and that of the reference model system may not switch at the same time.

Control objective. The control objective is to develop a state feedback MRAC scheme for the system (5.5.1) such that all signals in closed loop are bounded, and the plant state $\boldsymbol{x}(t)$ asymptotically tracks the reference trajectory $x_{m}(t)$ generated from (5.5.2), i.e.,

$$
\lim _{t \rightarrow \infty}\left(\boldsymbol{x}(t)-\boldsymbol{x}_{m}(t)\right)=\mathbf{0}
$$

\subsubsection{Adaptive Control Design}

A state feedback MRAC scheme is proposed in this section. Indicator functions are defined to characterize the switching behavior of the system (5.5.1) as well as that of the reference model (5.5.2). As a motivation to the adaptive control design, the model reference control problem is considered first.

\subsubsection{Indicator Functions}

We define the indicator functions $\chi_{\sigma}(t), \sigma \in\{1,2,3,4\}$, as follows:

$$
\begin{aligned}
& \chi_{\sigma}(t)=1, \text { if } \boldsymbol{x}(t) \in \Omega_{i} \text { and } \boldsymbol{x}_{m}(t) \in \Omega_{m j} \\
& \sigma= \begin{cases}1, & \text { iff } i=1, j=1 \\
2, & \text { iff } i=2, j=1 \\
3, & \text { iff } i=2, j=2 \\
4, & \text { iff } i=1, j=2\end{cases} \\
& \chi_{\sigma}(t)=0, \text { otherwise }
\end{aligned}
$$


Since the system or the reference model state at a specific time instant can only belong to one and only one polyhedral cell, it follows for $i, j \in\{1,2,3,4\}$ that

$$
\begin{aligned}
& \chi_{i}(t) \chi_{j}(t)=0, \quad i \neq j \\
& \chi_{i}(t) \chi_{i}(t)=1, \quad \text { if } \chi_{i}(t)=1 \\
& \sum_{i=1}^{4} \chi_{i}(t)=1
\end{aligned}
$$

With the definition of the indicator functions $\chi_{\sigma}(t)$, the system dynamics in $(5.5 .1)$ can be rewritten as

$$
\begin{gathered}
\dot{\boldsymbol{x}}=\boldsymbol{A}(t) \boldsymbol{x}+\boldsymbol{b}(t) u+\boldsymbol{b}^{0}(t) \\
\boldsymbol{A}(t)=\boldsymbol{A}_{1}\left(\chi_{1}+\chi_{4}\right)+\boldsymbol{A}_{2}\left(\chi_{2}+\chi_{3}\right) \\
\boldsymbol{b}(t)=\boldsymbol{b}_{1}\left(\chi_{1}+\chi_{4}\right)+\boldsymbol{b}_{2}\left(\chi_{2}+\chi_{3}\right) \\
\boldsymbol{b}^{0}(t)=\boldsymbol{b}_{1}^{0}\left(\chi_{1}+\chi_{4}\right)+\boldsymbol{b}_{2}^{0}\left(\chi_{2}+\chi_{3}\right)
\end{gathered}
$$

and similarly for the reference model system (5.5.2):

$$
\begin{gathered}
\dot{\boldsymbol{x}}_{m}=\boldsymbol{A}_{m}(t) \boldsymbol{x}_{m}+\boldsymbol{b}_{m}(t) r+\boldsymbol{b}_{m}^{0}(t) \\
\boldsymbol{A}_{m}(t)=\boldsymbol{A}_{m 1}\left(\chi_{1}+\chi_{2}\right)+\boldsymbol{A}_{m 2}\left(\chi_{3}+\chi_{4}\right) \\
\boldsymbol{b}_{m}(t)=\boldsymbol{b}_{m 1}\left(\chi_{1}+\chi_{2}\right)+\boldsymbol{b}_{m 2}\left(\chi_{3}+\chi_{4}\right) \\
\boldsymbol{b}_{m}^{0}(t)=\boldsymbol{b}_{m 1}^{0}\left(\chi_{1}+\chi_{2}\right)+\boldsymbol{b}_{m 2}^{0}\left(\chi_{3}+\chi_{4}\right)
\end{gathered}
$$

Assumptions. For adaptive control design, we make the following assumptions:

(A5.5-1) There exists symmetric, positive definite matrices $\boldsymbol{P}_{m} \in \mathbb{R}^{n \times n}$ such that

$$
\boldsymbol{A}_{m i}^{\top} \boldsymbol{P}_{m}+\boldsymbol{P}_{m} \boldsymbol{A}_{m i}=-\boldsymbol{Q}_{m i}, \quad i=1,2
$$

for some $\boldsymbol{Q}_{m i} \in \mathbb{R}^{n \times n}, \boldsymbol{Q}_{m i}=\boldsymbol{Q}_{m i}^{\top}>\mathbf{0}$;

(A5.5-2) There exist $\boldsymbol{k}_{x \sigma}^{*}, k_{r \sigma}^{*}, k_{\sigma}^{0 *}, \sigma=1,2,3,4$, such that

$$
\boldsymbol{A}_{i}+\boldsymbol{b}_{i} \boldsymbol{k}_{x \sigma}^{* \top}=\boldsymbol{A}_{m j}, \boldsymbol{b}_{i} k_{r \sigma}^{*}=\boldsymbol{b}_{m j}, \boldsymbol{b}_{i} k_{\sigma}^{0 *}+\boldsymbol{b}_{i}^{0}=\boldsymbol{b}_{m j}^{0}
$$

with $\sigma, i, j$ being related as in (5.5.5); and

(A5.5-3) $\operatorname{sign}\left[k_{r \sigma}^{*}\right]$, the signs of $k_{r \sigma}^{*}$, are known. 


\subsubsection{Model Reference Control}

When the system parameter matrices $\boldsymbol{A}_{i}, \boldsymbol{b}_{i}, \boldsymbol{b}_{i}^{0}, i=1,2$, are all known, the following model reference controller is applied to achieve the control objective:

$$
\begin{aligned}
u(t) & =\boldsymbol{k}_{x}^{* \boldsymbol{T}}(t) \boldsymbol{x}(t)+k_{r}^{*}(t) r(t)+k^{0 *}(t) \\
\boldsymbol{k}_{x}^{*}(t) & =\sum_{\sigma=1}^{4} \boldsymbol{k}_{x \sigma}^{*} \chi_{\sigma}(t), \quad k_{r}^{*}(t)=\sum_{\sigma=1}^{4} k_{r \sigma}^{*} \chi_{\sigma}(t), \quad k^{0 *}(t)=\sum_{\sigma=1}^{4} k_{\sigma}^{0 *} \chi_{\sigma}(t)
\end{aligned}
$$

where $\boldsymbol{k}_{x \sigma}^{*}, k_{r \sigma}^{*}, k_{\sigma}^{0 *}, \sigma=1,2,3,4$, are defined to satisfy Assumption (A5.5-2).

The equations in (5.5.10) are called the plant-model matching conditions, under which with (5.5.11) in (5.5.7) and the indicator function properties (5.5.6), the closedloop system matches the reference model system, leading to the closed-loop error dynamics as

$$
\dot{\boldsymbol{e}}(t)=\boldsymbol{A}_{m}(t) \boldsymbol{e}(t), \quad \boldsymbol{e}(t)=\boldsymbol{x}(t)-\boldsymbol{x}_{m}(t)
$$

With the Lyapunov function candidate $V=\boldsymbol{e}^{\top}(t) \boldsymbol{P}_{m} \boldsymbol{e}(t)$, it follows that $\dot{V} \leq-\lambda \boldsymbol{e}^{\top} \boldsymbol{e}$, where $\lambda$ is the minimum eigenvalue of the matrices $\boldsymbol{Q}_{m i}, i=1,2$, in (5.5.9). It can be concluded that $\boldsymbol{e}(t) \in \mathcal{L}^{2}$ and all closed-loop signals are bounded. In addition, $\dot{\boldsymbol{e}}(t) \in \mathcal{L}^{\infty}$ so that $\lim _{t \rightarrow \infty} \boldsymbol{e}(t)=\mathbf{0}$.

\subsubsection{Adaptive Control Design}

In general the system parameter matrices are unknown so that the model reference controller (5.5.11) cannot be implemented. To deal with system parameter uncertainties, an adaptive control approach is proposed.

Controller Structure. The following adaptive controller structure is applied:

$$
u(t)=\boldsymbol{k}_{x}^{\top}(t) \boldsymbol{x}(t)+k_{r}(t) r(t)+k^{0}(t)
$$

with $\boldsymbol{k}_{x}(t), k_{r}(t), k^{0}(t)$ being the time-varying estimates of $\boldsymbol{k}_{x}^{*}(t), k_{r}^{*}(t), k^{0 *}(t)$ and 
assuming the form:

$$
\boldsymbol{k}_{x}(t)=\sum_{\sigma=1}^{4} \boldsymbol{k}_{x \sigma}(t) \chi_{\sigma}(t), \quad k_{r}(t)=\sum_{\sigma=1}^{4} k_{r \sigma}(t) \chi_{\sigma}(t), \quad k^{0}(t)=\sum_{\sigma=1}^{4} k_{\sigma}^{0}(t) \chi_{\sigma}(t)
$$

Here $\boldsymbol{k}_{x \sigma}(t), k_{r \sigma}(t), k_{\sigma}^{0}(t)$ are respectively the estimates of $\boldsymbol{k}_{x \sigma}^{*}, k_{r \sigma}^{*}, k_{\sigma}^{0 *}$, defined in $(5.5 .10)$.

Error Model. With the control law (5.5.12) applied to the system (5.5.7), and from the reference model system (5.5.8), the closed-loop error dynamics is obtained as follows:

$$
\begin{aligned}
\dot{\boldsymbol{e}} & =\boldsymbol{A}_{m} \boldsymbol{e}+\boldsymbol{b} \tilde{\boldsymbol{k}}_{x}^{\top} \boldsymbol{x}+\boldsymbol{b} \tilde{k}_{r} \boldsymbol{r}+\boldsymbol{b} \tilde{k}^{0} \\
& =\boldsymbol{A}_{m} \boldsymbol{e}+\sum_{\sigma=1}^{4} \frac{\boldsymbol{b}_{m j}}{k_{r \sigma}^{*}}\left(\tilde{\boldsymbol{k}}_{x \sigma}^{\top} \chi_{\sigma} \boldsymbol{x}+\tilde{k}_{r \sigma} \chi_{\sigma} r+\tilde{k}_{\sigma}^{0} \chi_{\sigma}\right)
\end{aligned}
$$

where $j$ is defined through (5.5.5), and $\tilde{\boldsymbol{k}}_{x \sigma}(t)=\boldsymbol{k}_{x \sigma}(t)-\boldsymbol{k}_{x \sigma}^{*}, \tilde{k}_{r \sigma}(t)=k_{r \sigma}(t)-k_{r \sigma}^{*}$, $\tilde{k}_{\sigma}^{0}(t)=k_{\sigma}^{0}(t)-k_{\sigma}^{0 *}$.

Adaptive Laws. We propose to apply the following adaptive laws to update the controller parameters in (5.5.12):

$$
\begin{aligned}
\dot{\boldsymbol{k}}_{x \sigma}(t) & =-\operatorname{sign}\left[k_{r \sigma}^{*}\right] \boldsymbol{\Gamma}_{\sigma} \boldsymbol{x}(t) \boldsymbol{e}^{\top}(t) \boldsymbol{P}_{m} \boldsymbol{b}_{m j} \chi_{\sigma}(t) \\
\dot{k}_{r \sigma}(t) & =-\operatorname{sign}\left[k_{r \sigma}^{*}\right] \gamma_{\sigma} r(t) \boldsymbol{e}^{\top}(t) \boldsymbol{P}_{m} \boldsymbol{b}_{m j} \chi_{\sigma}(t) \\
\dot{k}_{\sigma}^{0}(t) & =-\operatorname{sign}\left[k_{r \sigma}^{*}\right] \gamma_{\sigma}^{0} \boldsymbol{e}^{\top}(t) \boldsymbol{P}_{m} \boldsymbol{b}_{m j} \chi_{\sigma}(t)
\end{aligned}
$$

where $\boldsymbol{\Gamma}_{\sigma}=\boldsymbol{\Gamma}_{\sigma}^{\boldsymbol{\top}}>\mathbf{0}, \gamma_{\sigma}>0, \gamma_{\sigma}^{0}>0$ are the adaptation gain matrices (gains), $j$ is related to $\sigma$ through $(5.5 .5)$, and the initial parameter estimates $\boldsymbol{k}_{x \sigma}(0), k_{r \sigma}(0), k_{\sigma}^{0}(0)$ may be chosen arbitrarily.

The following closed-loop stability properties and tracking performance follow:

Theorem 5.5.1. Consider the closed-loop system with the controlled plant (5.5.7), the reference model system (5.5.8), the adaptive control law (5.5.12) with parameters updated from the adaptive laws (5.5.14). All signals in the closed-loop system are 
bounded and asymptotic state tracking is achieved, i.e.,

$$
\lim _{t \rightarrow \infty}\left(\boldsymbol{x}(t)-\boldsymbol{x}_{m}(t)\right)=\mathbf{0}
$$

Proof: Consider the continuous Lyapunov function candidate

$$
V=\boldsymbol{e}^{\boldsymbol{\top}} \boldsymbol{P}_{m} \boldsymbol{e}+\sum_{\sigma=1}^{4} \frac{1}{\left|k_{r \sigma}^{*}\right|}\left(\tilde{\boldsymbol{k}}_{x \sigma}^{\top} \boldsymbol{\Gamma}_{\sigma}^{-1} \tilde{\boldsymbol{k}}_{x \sigma}+\frac{1}{\gamma_{\sigma}} \tilde{k}_{r \sigma}^{2}+\frac{1}{\gamma_{\sigma}^{0}} \tilde{k}_{\sigma}^{02}\right)
$$

Its time derivative along (5.5.13) and (5.5.14) is

$$
\begin{aligned}
\dot{V}= & \boldsymbol{e}^{\boldsymbol{\top}}\left(\boldsymbol{A}_{m}^{\top} \boldsymbol{P}_{m}+\boldsymbol{P}_{m} \boldsymbol{A}_{m}\right) \boldsymbol{e}+\sum_{\sigma=1}^{4} \frac{2}{k_{r \sigma}^{*}} \boldsymbol{e}^{\boldsymbol{\top}} \boldsymbol{P}_{m} \boldsymbol{b}_{m j}\left(\tilde{\boldsymbol{k}}_{x \sigma}^{\boldsymbol{\top}} \chi_{\sigma} \boldsymbol{x}+\tilde{k}_{r \sigma} \chi_{\sigma} r+\tilde{k}_{\sigma}^{0} \chi_{\sigma}\right) \\
& +\sum_{\sigma=1}^{4} \frac{2}{\left|k_{r \sigma}^{*}\right|}\left(\tilde{\boldsymbol{k}}_{x \sigma}^{\boldsymbol{\top}} \boldsymbol{\Gamma}_{\sigma}^{-1} \dot{\boldsymbol{k}}_{x \sigma}+\frac{1}{\gamma_{\sigma}} \tilde{k}_{r \sigma} \dot{k}_{r \sigma}+\frac{1}{\gamma_{\sigma}^{0}} \tilde{k}_{\sigma}^{0} \dot{k}_{\sigma}^{0}\right) \\
\leq & -\lambda \boldsymbol{e}^{\boldsymbol{\top}} \boldsymbol{e}
\end{aligned}
$$

where $\lambda$ is the minimum eigenvalue of the matrices $\boldsymbol{Q}_{m i}, i=1,2$, in (5.5.9). It can be concluded that $\boldsymbol{e}(t) \in \mathcal{L}^{2}$ and $\boldsymbol{e}(t), \boldsymbol{k}_{x \sigma}(t), k_{r \sigma}(t), k_{\sigma}^{0}(t) \in \mathcal{L}^{\infty}$. From $\boldsymbol{e}(t)=\boldsymbol{x}(t)-$ $\boldsymbol{x}_{m}(t)$ and (5.5.12), we have $\boldsymbol{x}(t), u(t) \in \mathcal{L}^{\infty}$. Furthermore, it follows from (5.5.13) that $\dot{\boldsymbol{e}}(t) \in \mathcal{L}^{\infty}$. Therefore, all closed-loop signals are bounded and asymptotic state tracking is achieved: $\lim _{t \rightarrow \infty} \boldsymbol{e}(t)=\mathbf{0}$.

\subsubsection{Simulation Study}

Simulations are performed to demonstrate the system stability and tracking performance with the proposed adaptive control schemes applied to a mass-spring-damper system as shown in Fig. 5.6.

Assume that all elements are linear, and there is no friction between the cart with mass $m$ and the ground. Choose a reference point as the right tip of the one-sided spring with spring constant $k_{2}$ when it is relaxed (as shown by the leftmost vertical dotted line in Fig. 5.6). Suppose that the relaxed position of the right tip of the spring with constant $k_{1}$ is $d$ units of length to the right of the reference point. Let 


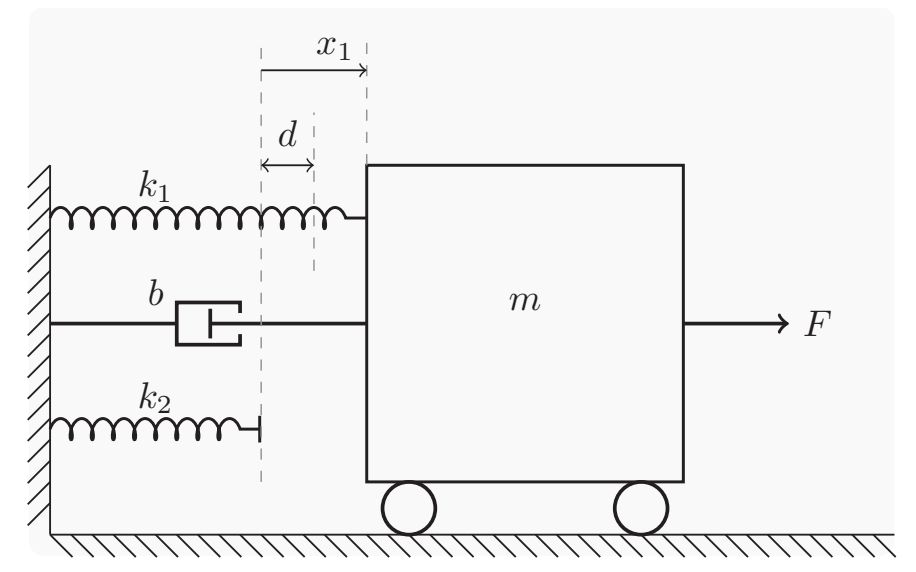

Figure 5.6: Mass-spring-damper system with a one-sided spring.

$x_{1}$ denote the displacement of the cart from this reference point, and $F$ an external force.

The system dynamics can be described by the following differential equation:

$$
F+k_{1}\left(-x_{1}+d\right)-b \dot{x}_{1}+k_{2} \max \left\{-x_{1}, 0\right\}=m \ddot{x}_{1}
$$

With $x_{2} \triangleq \dot{x}_{1}$ and $\boldsymbol{x}=\left[x_{1}, x_{2}\right]^{\top}$, the state space model of the system is in the form of (5.5.1); in particular,

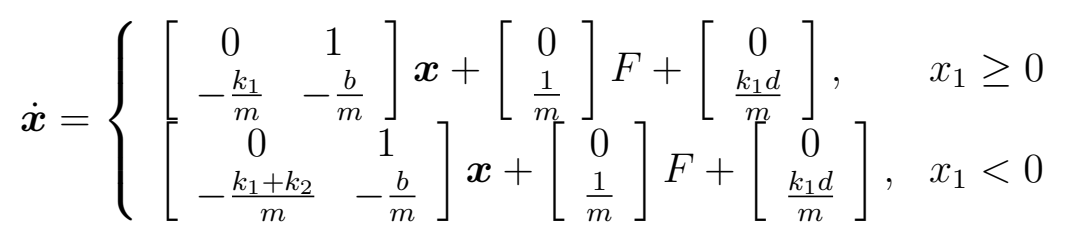

The switching hyperplane is thus defined with $\boldsymbol{H}=[1,0]^{\top}$ and $h=0$.

Due to the fact that $\boldsymbol{A}_{i}, \boldsymbol{b}_{i}, \boldsymbol{b}_{i}^{0}, i=1,2$, are all in controllable canonical form, it is straightforward to verify that nominal parameters $\boldsymbol{k}_{x \sigma}^{*}, k_{r \sigma}^{*}, k_{\sigma}^{0 *}, \sigma=1,2,3,4$, always exist such that the matching condition (5.5.10) is satisfied.

Two simulation studies are performed based on the choices of two different reference model systems and the plant parameters: $m=1, k_{1}=1, k_{2}=0.5, b=0.2$, $d=0.5$. The initial plant state is $\boldsymbol{x}(0)=[-2,2]^{\top}$. The adaptive control scheme 
is configured with the initial parameter estimates being $90 \%$ of their nominal values and $\boldsymbol{\Gamma}_{\sigma}=0.5 \boldsymbol{I}_{2}, \gamma_{\sigma}=\gamma_{\sigma}^{0}=0.5, \sigma=1,2,3,4$.

Simulation I. The reference model system is chose to be linear time-invariant as follows:

$$
\dot{\boldsymbol{x}}_{m}=\left[\begin{array}{cc}
0 & 1 \\
-0.5 & -1
\end{array}\right] \boldsymbol{x}_{m}+\left[\begin{array}{l}
0 \\
1
\end{array}\right] r, \quad \boldsymbol{x}_{m}(0)=\left[\begin{array}{l}
-3 \\
-3
\end{array}\right]
$$

where the reference input signal is $r(t)=2 \sin (2 \pi 0.1 t)$. The $\boldsymbol{P}_{m}$ matrix in the adaptive laws (5.5.14) is

$$
\boldsymbol{P}_{m}=\left[\begin{array}{ll}
47.1825 & 23.5913 \\
23.5913 & 47.1825
\end{array}\right]
$$

With such a reference model system, the objective is to make the closed-loop system behave as if the cart is attached to a damper with damping constant 1 and a single spring with constant 0.5 , whose relaxed position is the same as the chosen reference point in Fig. 5.6. The state tracking error $\boldsymbol{e}(t)$ is plotted in Fig. 5.7. It is clear that asymptotic state tracking is achieved. In addition, all closed-loop signals are bounded.

Simulation II. The reference model system is chose to be piecewise linear with dynamic offsets as in (5.5.2) with

$$
\begin{aligned}
& \boldsymbol{A}_{m 1}=\left[\begin{array}{cc}
0 & 1 \\
-0.5 & -1
\end{array}\right], \quad \boldsymbol{b}_{m 1}=\left[\begin{array}{l}
0 \\
1
\end{array}\right], \quad \boldsymbol{b}_{m 1}^{0}=\left[\begin{array}{c}
0 \\
-0.5
\end{array}\right] \\
& \boldsymbol{A}_{m 2}=\left[\begin{array}{cc}
0 & 1 \\
-1 & -5
\end{array}\right], \quad \boldsymbol{b}_{m 2}=\left[\begin{array}{l}
0 \\
1
\end{array}\right], \quad \boldsymbol{b}_{m 2}^{0}=\left[\begin{array}{l}
0 \\
2
\end{array}\right]
\end{aligned}
$$

The switching hyperplane is defined with $\boldsymbol{H}_{m}=[1,0]^{\top}, h_{m}=0$, and the $\boldsymbol{P}_{m}$ matrix used in the adaptive laws is

$$
\boldsymbol{P}_{m}=\left[\begin{array}{ll}
6.3849 & 1.9503 \\
1.9503 & 4.5967
\end{array}\right]
$$

which can be verified to satisfy Assumption (A5.5-1). As for Simulation I, closed-loop signal boundedness and asymptotic state tracking performance are achieved. 

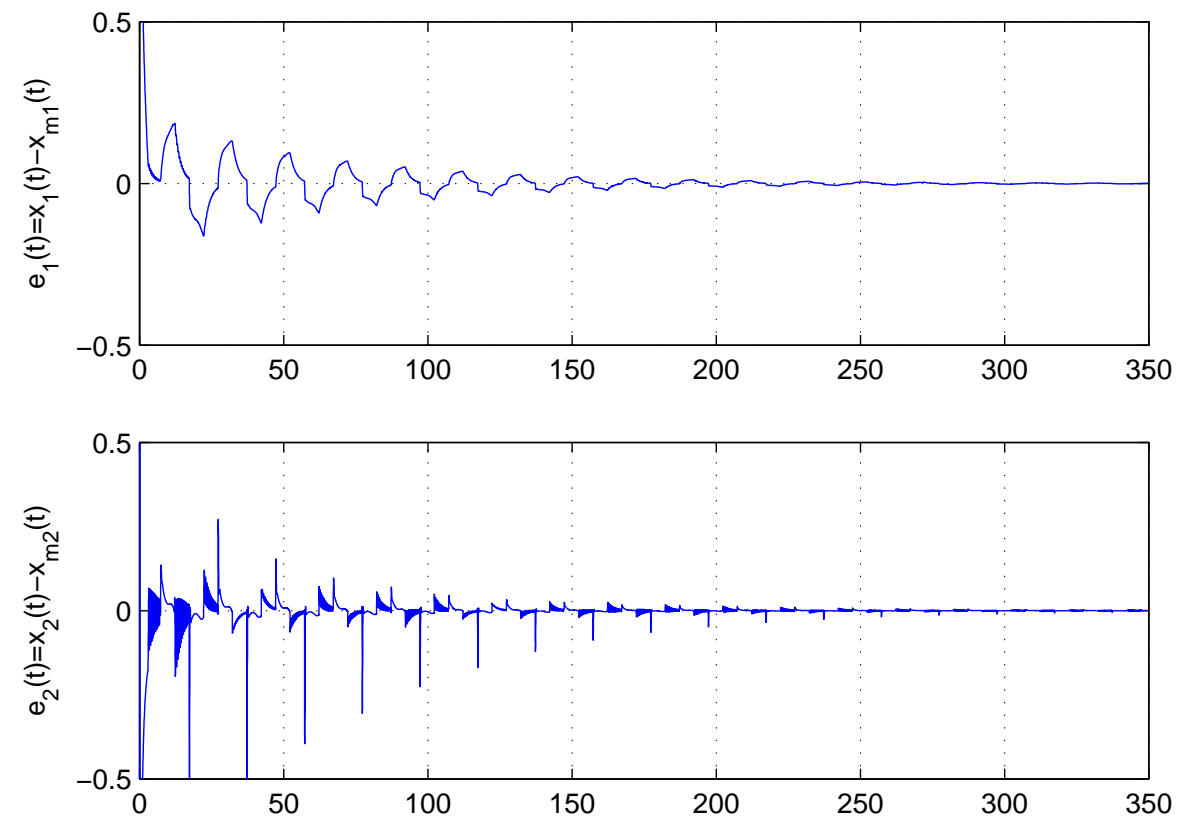

Figure 5.7: State tracking error $e(t)$ for Simulation I: the LTI reference model system in $(5.5 .15)$.
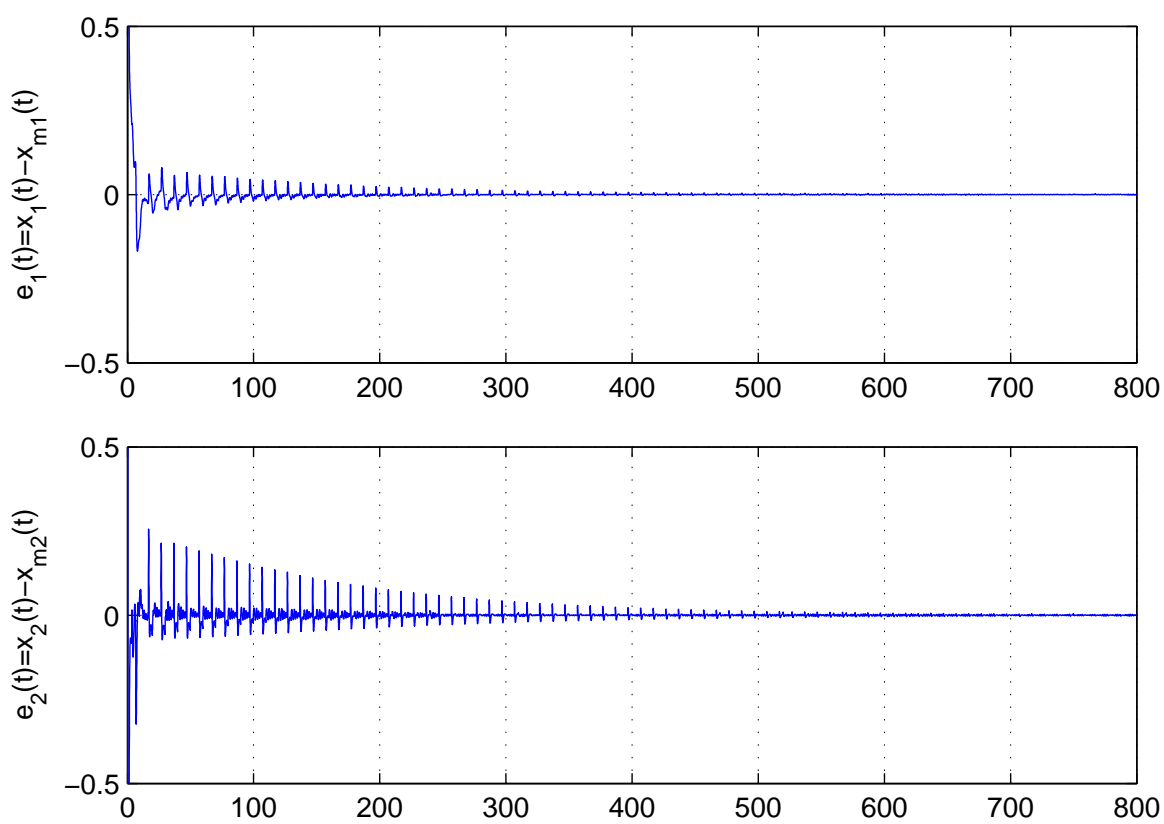

Figure 5.8: State tracking error $e(t)$ for Simulation II: the piecewise linear reference model system (5.5.2) with parameter matrices as in (5.5.16). 


\section{Chapter 6}

\section{Adaptive Control of Piecewise Linear Systems with State Feedback for Output Tracking}

In this chapter, we study the adaptive state feedback for output tracking control problem for piecewise linear systems. Direct model reference adaptive control (MRAC) schemes are developed for both single-input, single-output (SISO) and multiple-input, multiple-output (MIMO) piecewise linear systems. It is shown that with such MRAC schemes, closed-loop stability (signal boundedness) and small output tracking error are achieved, if the occurrence frequency of parameter discontinuities is sufficiently low. The desired asymptotic tracking performance is achieved for arbitrarily frequent parameter discontinuities under certain matching conditions, e.g., piecewise linear systems in controllable canonical form (CCF). As compared to the designs in Chapter 5 for state tracking, we will present state feedback for output tracking designs which has a less restrictive plant-model matching condition. Their stability and tracking performance are analyzed and evaluated by simulation examples.

This chapter is organized as follows. The adaptive SFOT control designs for SISO piecewise linear systems are proposed and analyzed in Section 6.1. Multivariable designs are presented in Section 6.2. Illustrative examples to demonstrate the 
effectiveness of the proposed adaptive control schemes are provided in Section 6.3.

\subsection{Design for SISO Piecewise Linear Systems}

The adaptive state feedback control problem is studied in this section for a SISO piecewise linear system to make its output track a desired trajectory generated from a linear time-invariant (LTI) reference model system.

\subsubsection{Problem Statement}

Consider a SISO piecewise linear system:

$$
\begin{gathered}
\dot{\boldsymbol{x}}(t)=\boldsymbol{A}(t) \boldsymbol{x}(t)+\boldsymbol{b}(t) u(t) \\
y(t)=\boldsymbol{c}^{\top} \boldsymbol{x}(t), \quad \boldsymbol{x}(0)=\boldsymbol{x}_{0}
\end{gathered}
$$

where $\boldsymbol{x}(t) \in \mathbb{R}^{n}$ is the state vector and is available for measurement, $u(t) \in \mathbb{R}$ is the control input, $y(t) \in \mathbb{R}$ is the controlled output, $\boldsymbol{A}(t) \in \mathbb{R}^{n \times n}$ and $\boldsymbol{b}(t) \in \mathbb{R}^{n}$ are unknown time-varying system parameter matrices, and $\boldsymbol{c} \in \mathbb{R}^{n}$ is an unknown constant parameter vector. The parameters matrices $\boldsymbol{A}(t)$ and $\boldsymbol{b}(t)$ vary in a piecewise linear pattern; that is, during different time periods, $(\boldsymbol{A}(t), \boldsymbol{b}(t))$ take on different values as specified by the parameter matrix sets $\left(\boldsymbol{A}_{i}, \boldsymbol{b}_{i}\right)$, called a subysystme of (6.1.1), $i \in \mathcal{I} \triangleq\{1,2, \ldots, l\}$, where $\boldsymbol{A}_{i}$ and $\boldsymbol{b}_{i}$ are unknown but constant parameter matrices, and $l$ is the total number of subsystems. To characterize such time-varying behaviors of the system, the indicator functions are introduced.

Indicator Functions. The knowledge of the durations of time of the $i$ th subsystem that the system assumes and the time instants at which it switches to the $j$ th, $i, j \in \mathcal{I}$, is crucial for adaptive control design. The indicator functions $\chi_{i}(t)$, which contain such knowledge of the system parameter discontinuities, are assumed to be 
known and defined as

$$
\chi_{i}(t)= \begin{cases}1, & \text { if }(\boldsymbol{A}(t), \boldsymbol{b}(t))=\left(\boldsymbol{A}_{i}, \boldsymbol{b}_{i}\right) \\ 0, & \text { otherwise }\end{cases}
$$

It follows that

$$
\sum_{i=1}^{l} \chi_{i}(t)=1 \quad \chi_{j}(t) \chi_{k}(t)=0, \quad j \neq k
$$

With the indicator functions $\chi_{i}(t)$, the time-varying plant parameter matrices $\boldsymbol{A}(t)$ and $\boldsymbol{b}(t)$ can be expressed as

$$
\boldsymbol{A}(t)=\sum_{i=1}^{l} \boldsymbol{A}_{i} \chi_{i}(t), \quad \boldsymbol{b}(t)=\sum_{i=1}^{l} \boldsymbol{b}_{i} \chi_{i}(t)
$$

Control Objective. The control objective is to develop a feedback control law $u(t)$ for the system (6.1.1) with parameter variations characterized as in (6.1.3) such that all the signals in the closed-loop system are bounded, and the plant output $y(t)$ tracks a reference signal $y_{m}(t)$ as closely as possible, with $y_{m}(t)$ generated from a reference model system

$$
y_{m}(t)=W_{m}(s)[r](t), \quad W_{m}(s)=\frac{1}{P_{m}(s)}
$$

where $P_{m}(s)$, independent of the system parameters, is a desired stable closed-loop characteristic polynomial of degree $n^{*}$, and $r(t)$ is an external reference input signal which is bounded and piecewise continuous.

MRAC Assumptions. Suppose the transfer function for the $i$ th subsystem is

$$
G_{i}(s)=c^{\top}\left(s \boldsymbol{I}-\boldsymbol{A}_{i}\right)^{-1} \boldsymbol{b}_{i}=\frac{k_{p i} Z_{i}(s)}{P_{i}(s)}
$$

with $k_{p i} \neq 0$ a constant and $P_{i}(s)=\operatorname{det}\left(s \boldsymbol{I}-\boldsymbol{A}_{i}\right), Z_{i}(s)$ being monic polynomials with unknown constant coefficients and of degrees $n$ and $m$, respectively. To design an adaptive state feedback control law for output tracking, the following assumptions are made for $i \in \mathcal{I}$ : 
(A6.1-1) $\left(\boldsymbol{c}^{\top}, \boldsymbol{A}_{i}, \boldsymbol{b}_{i}\right)$ is stabilizable and detectible;

(A6.1-2) The zeros of $Z_{i}(s)$ are stable with their real parts less than $-\delta$ for some known $\delta>0$;

(A6.1-3) The degree $m$ of $Z_{i}(s)$ is known;

(A6.1-4) The degree of $P_{m}(s)$ is $n^{*}=n-m$; and

(A6.1-5) The sign of $k_{p i}, \operatorname{sign}\left[k_{p i}\right]$, is known.

Remark 6.1.1. The knowledge of $\delta$ in Assumption (A6.1-2) is required such that a suitable dynamic normalizing signal can be designed, and the boundedness property of the normalized system state vector can be used in concluding the closed-loop stability properties [11].

Next, the non-adaptive nominal model reference control problem is considered first, which motivates the adaptive control scheme presented in Section 6.1.3.

\subsubsection{Nominal Control Scheme}

Controller Structure. With complete knowledge of plant parameter matrices $\boldsymbol{A}_{i}$ and $\boldsymbol{b}_{i}, i \in \mathcal{I}$, the following state feedback controller structure is applied

$$
u(t)=\boldsymbol{k}_{x}^{* \top}(t) \boldsymbol{x}(t)+k_{r}^{*}(t) r(t)
$$

with the time-varying nominal controller parameters defined as

$$
\begin{aligned}
\boldsymbol{k}_{x}^{*}(t) & =\boldsymbol{k}_{x 1}^{*} \chi_{1}(t)+\boldsymbol{k}_{x 2}^{*} \chi_{2}(t)+\cdots+\boldsymbol{k}_{x l}^{*} \chi_{l}(t) \\
k_{r}^{*}(t) & =k_{r 1}^{*} \chi_{1}(t)+k_{r 2}^{*} \chi_{2}(t)+\cdots+k_{r l}^{*} \chi_{l}(t)
\end{aligned}
$$

and constant vectors $\boldsymbol{k}_{x i}^{*} \in \mathbb{R}^{n}$ and $k_{r i}^{*} \in \mathbb{R}$ computed from the piecewise plant-model matching condition:

$$
\operatorname{det}\left(s \boldsymbol{I}-\boldsymbol{A}_{i}-\boldsymbol{b}_{i} \boldsymbol{k}_{x i}^{* \boldsymbol{T}}\right)=P_{m}(s) Z_{i}(s), \quad k_{r i}^{*}=\frac{1}{k_{p i}}
$$


It is "piecewise" in the sense that when the $i$ th subsystem is active (as indicated by $\left.\chi_{i}(t)=1\right)$, the controller (6.1.6) leads to an input/output transfer function matching of the closed-loop system $\left(\boldsymbol{c}^{\top}, \boldsymbol{A}_{m i}, \boldsymbol{b}_{m i}\right)$ with

$$
\boldsymbol{A}_{m i} \triangleq \boldsymbol{A}_{i}+\boldsymbol{b}_{i} \boldsymbol{k}_{x i}^{* \top}, \quad \boldsymbol{b}_{m i} \triangleq \boldsymbol{b}_{i} k_{r i}^{*}
$$

to the reference model system, i.e.,

$$
\boldsymbol{c}^{\boldsymbol{\top}}\left(s \boldsymbol{I}-\boldsymbol{A}_{m i}\right)^{-1} \boldsymbol{b}_{m i}=\frac{k_{p i} Z_{i}(s) k_{r i}^{*}}{\operatorname{det}\left(s \boldsymbol{I}-\boldsymbol{A}_{m i}\right)}=W_{m}(s)
$$

The existence of such $\boldsymbol{k}_{x i}^{*}$ and $k_{r i}^{*}$ is guaranteed by Assumptions (A6.1-1) and (A6.12). Furthermore, the matrices $\boldsymbol{A}_{m i}, i \in \mathcal{I}$, are all stable, since both $P_{m}(s)$ and $Z_{i}(s)$ are stable polynomials by assumption.

Let the increasing sequence $\left\{t_{k}\right\}_{k=1}^{\infty}$ denote the time instants at which subsystem switches occur. With (6.1.6) in (6.1.1), the closed-loop system becomes

$$
\dot{\boldsymbol{x}}(t)=\sum_{i=1}^{l}\left[\boldsymbol{A}_{m i} \chi_{i}(t) \boldsymbol{x}(t)+\boldsymbol{b}_{m i} \chi_{i}(t) r(t)\right], \quad y(t)=\boldsymbol{c}^{\top} \boldsymbol{x}(t)
$$

It is worth noting that unlike conventional state feedback MRC developed for LTI systems for output tracking [15], the nominal control law (6.1.6) designed based on the piecewise matching equations (6.1.7) may not ensure stability without imposing additional conditions on subsystem switches (e.g., the minimum switching time interval requirement on $T_{0}$ in (5.2.4) of Lemma 5.2.1. Even if the closed-loop system is stable, we cannot expect asymptotic tracking performance. This is because the piecewise matching equation (6.1.7) does not incorporate the boundary conditions at each subsystem switching time instant, determined by the internal state of the closed-loop system. The closed-loop stability properties and the output tracking performance of the nominal closed-loop system are summarized next. 
Stability Properties. Let $\left(\boldsymbol{c}^{\top}, \boldsymbol{A}_{m(k-1)}, \boldsymbol{b}_{m(k-1)}\right) \in\left\{\left(\boldsymbol{c}^{\top}, \boldsymbol{A}_{m i}, \boldsymbol{b}_{m i}\right)\right\}_{i=1}^{l}$ denote the active subsystem over $\left[t_{k-1}, t_{k}\right), k \in \mathbb{Z}^{+}, \mathbf{\Phi}(t, \tau)$ the state transition matrix associated with the homogeneous system

$$
\dot{\boldsymbol{z}}(t)=\boldsymbol{A}_{m}(t) \boldsymbol{z}(t), \quad \boldsymbol{A}_{m}(t)=\sum_{i=1}^{l} \boldsymbol{A}_{m i} \chi_{i}(t)
$$

and $\left(\boldsymbol{c}_{m}^{\top}, \boldsymbol{A}_{m}, \boldsymbol{b}_{m}\right)$ a state space realization of the reference model system (6.1.4) with the associated state transition matrix $\boldsymbol{\Phi}_{m}(t, \tau)$. For the nominal closed-loop system, consisting of the plant (6.1.1), the reference model (6.1.4), and the controller (6.1.6), we have the following results:

Theorem 6.1.2. All signals in the nominal closed-loop system are bounded if the minimum switching time interval $T_{0}$ satisfies (5.2.4). The output tracking error $e(t)=$ $y(t)-y_{m}(t)$ is

$$
e(t)=\eta_{0}(t)+\varepsilon_{0}(t)
$$

with $\varepsilon_{0}(t)$ an initial condition related term

$$
\varepsilon_{0}(t)=\boldsymbol{c}^{\top} \boldsymbol{\Phi}\left(t, t_{0}\right) \boldsymbol{x}\left(t_{0}\right)-\boldsymbol{c}_{m}^{\top} \boldsymbol{\Phi}_{m}\left(t, t_{0}\right) \boldsymbol{x}_{m}\left(t_{0}\right)
$$

which is exponentially decaying, and for $t \in\left[t_{k-1}, t_{k}\right)$,

$$
\eta_{0}(t)=\sum_{j=2}^{k} \int_{t_{j-2}}^{t_{j-1}}\left[\boldsymbol{c}^{\top} \boldsymbol{\Phi}(t, \tau) \boldsymbol{b}_{m(j-2)}-\boldsymbol{c}_{m}^{\top} \boldsymbol{\Phi}_{m}(t, \tau) \boldsymbol{b}_{m}\right] r(\tau) d \tau
$$

which is small in the mean square sense, under the slow switching condition (5.2.4). Furthermore, there exist positive constants $C_{1}$ and $K_{1}$, such that $e(t)$ satisfies

$$
\int_{t}^{t+T} e^{2}(\tau) d \tau \leq C_{1}+K_{1} n_{T}, \quad \forall t \geq t_{0}, \quad \forall T \geq 0
$$

where $n_{T}$ is the total number of subsystem switches over $[t, t+T]$. 
Proof: The signal boundedness properties of the closed-loop system can be proved following Lemma 5.2.1. Consider the state evolution inside a switching time interval $\left[t_{k-1}, t_{k}\right), k \in \mathbb{Z}^{+}$, over which we have

$$
\begin{gathered}
y(t)=\boldsymbol{c}^{\top} \boldsymbol{\Phi}\left(t, t_{k-1}\right) \boldsymbol{x}\left(t_{k-1}\right)+\boldsymbol{c}^{\top} \int_{t_{k-1}}^{t} \boldsymbol{\Phi}(t, \tau) \boldsymbol{b}_{m(k-1)} r(\tau) d \tau \\
y_{m}(t)=\boldsymbol{c}_{m}^{\top} \boldsymbol{\Phi}_{m}\left(t, t_{k-1}\right) \boldsymbol{x}_{m}\left(t_{k-1}\right)+\boldsymbol{c}_{m}^{\top} \int_{t_{k-1}}^{t} \boldsymbol{\Phi}_{m}(t, \tau) \boldsymbol{b}_{m} r(\tau) d \tau
\end{gathered}
$$

From (6.1.9) we can see that $\left(\boldsymbol{c}^{\top}, \boldsymbol{A}_{m(k-1)}, \boldsymbol{b}_{m(k-1)}\right)$ is a non-minimal realization of $W_{m}(s)=1 / P_{m}(s)$, so that

$$
\boldsymbol{c}^{\boldsymbol{\top}} \int_{t_{k-1}}^{t} \boldsymbol{\Phi}(t, \tau) \boldsymbol{b}_{m(k-1)} r(\tau) d \tau=\boldsymbol{c}_{m}^{\top} \int_{t_{k-1}}^{t} \boldsymbol{\Phi}_{m}(t, \tau) \boldsymbol{b}_{m} r(\tau) d \tau
$$

It then follows that

$$
\begin{aligned}
e(t)= & \boldsymbol{c}^{\top} \boldsymbol{\Phi}\left(t, t_{k-1}\right) \boldsymbol{x}\left(t_{k-1}\right)-\boldsymbol{c}_{m}^{\top} \boldsymbol{\Phi}_{m}\left(t, t_{k-1}\right) \boldsymbol{x}_{m}\left(t_{k-1}\right) \\
= & \boldsymbol{c}^{\boldsymbol{\top}}\left[\boldsymbol{\Phi}\left(t, t_{k-2}\right) \boldsymbol{x}\left(t_{k-2}\right)+\int_{t_{k-2}}^{t_{k-1}} \boldsymbol{\Phi}(t, \tau) \boldsymbol{b}_{m(k-2)} r(\tau) d \tau\right] \\
& -\boldsymbol{c}_{m}^{\boldsymbol{\top}}\left[\boldsymbol{\Phi}_{m}\left(t, t_{k-2}\right) \boldsymbol{x}_{m}\left(t_{k-2}\right)+\int_{t_{k-2}}^{t_{k-1}} \boldsymbol{\Phi}_{m}(t, \tau) \boldsymbol{b}_{m} r(\tau) d \tau\right] \\
& \vdots \\
= & \eta_{0}(t)+\varepsilon_{0}(t)
\end{aligned}
$$

with $\varepsilon_{0}(t)$ and $\eta_{0}(t)$ in (6.1.11) and (6.1.12), respectively.

From Lemma 5.2.1, the state transition matrix $\boldsymbol{\Phi}(t, \tau)$ associated with the homogeneous system is exponentially stable under (5.2.4). We can conclude that $\varepsilon_{0}(t)$ decays exponentially. The smallness of $\eta_{0}(t)$ in the mean square sense can be established by dividing the integration interval into corresponding switching time intervals, and noting the exponential stability of $\boldsymbol{\Phi}(t, \tau), \boldsymbol{\Phi}_{m}(t, \tau)$ and the boundedness of system parameters and $r(t)$. The mean square tracking performance (6.1.13) can be established in a similar way. 
Remark 6.1.3. Due to the exponential stability of $\boldsymbol{\Phi}(t, \tau)$ and $\boldsymbol{\Phi}_{m}(t, \tau)$, each integral under the summation in $\eta_{0}(t)$ decays exponentially. Therefore, if the system ceases to switch after some time, the output tracking error decays to zero exponentially fast, and $e(t) \in \mathcal{L}^{2}$ (let $t=0$ and $T=\infty$ in (6.1.13), and the number of subsystem switches over $[0, \infty), n_{\infty}$, is finite).

Remark 6.1.4. As a part of the tracking error $e(t), \eta_{0}(t)$ in (6.1.12) captures the deviations of $y(t)$ from $y_{m}(t)$ caused by subsystem switches. In particular, for each integral under the summation, the first term can be rewritten as $\boldsymbol{c}^{\top} \boldsymbol{\Phi}\left(t, t_{j-1}\right) \overline{\boldsymbol{x}}\left(t_{j-1}\right)$, with $\overline{\boldsymbol{x}}\left(t_{j-1}\right)=\int_{t_{j-2}}^{t_{j-1}} \mathbf{\Phi}\left(t_{j-1}, \tau\right) \boldsymbol{b}_{m(j-2)} r(\tau) d \tau$ denoting the part of the closed-loop system state at the switching time instant $t_{j-1}$, generated by the reference input $r(t)$ over $\left[t_{j-2}, t_{j-1}\right)$. This term represents the contribution of $\overline{\boldsymbol{x}}\left(t_{j-1}\right)$ to the system output $y(t)$ at time $t$. Due to a subsystem switch at $t_{j-1}$, it is not matched by a counterpart in $y_{m}(t)$, i.e., the second term, $\boldsymbol{c}_{m}^{\top} \boldsymbol{\Phi}_{m}\left(t, t_{j-1}\right) \overline{\boldsymbol{x}}_{m}\left(t_{j-1}\right)$ with $\overline{\boldsymbol{x}}_{m}\left(t_{j-1}\right)=$ $\int_{t_{j-2}}^{t_{j-1}} \boldsymbol{\Phi}_{m}\left(t_{j-1}, \tau\right) \boldsymbol{b}_{m} r(\tau) d \tau$. The difference is the deviation in output tracking error due to the switch at $t_{j-1}$.

\subsubsection{Adaptive Control Design}

Since the plant parameter matrices $\boldsymbol{A}_{i}$ and $\boldsymbol{b}_{i}, i \in \mathcal{I}$, are unknown, the nominal controller (6.1.6) cannot be implemented. An adaptive controller structure with its parameters updated by some adaptive laws is proposed in this section.

Controller Structure. The adaptive controller structure is proposed as follows:

$$
u(t)=\boldsymbol{k}_{x}^{\top}(t) \boldsymbol{x}(t)+k_{r}(t) r(t)
$$

where $\boldsymbol{k}_{x}(t) \in \mathbb{R}^{n}$ and $k_{r}(t) \in \mathbb{R}$ are defined as

$$
\boldsymbol{k}_{x}(t)=\sum_{i=1}^{l} \boldsymbol{k}_{x i}(t) \chi_{i}(t), \quad k_{r}(t)=\sum_{i=1}^{l} k_{r i}(t) \chi_{i}(t)
$$


The parameter vectors $\boldsymbol{k}_{x i}(t) \in \mathbb{R}^{n}$ and $k_{r i}(t) \in \mathbb{R}$ are the estimates of $\boldsymbol{k}_{x i}^{*}$ and $k_{r i}^{*}$, respectively, defined to satisfy (6.1.7), and they are updated by some adaptive laws to be developed next.

By applying the control law (6.1.15) to the plant (6.1.1) and denoting the controller parameter error vectors as $\tilde{\boldsymbol{k}}_{x}(t)=\boldsymbol{k}_{x}(t)-\boldsymbol{k}_{x}^{*}(t), \tilde{k}_{r}(t)=k_{r}(t)-k_{r}^{*}(t), \tilde{\boldsymbol{k}}_{x i}(t)=$ $\boldsymbol{k}_{x i}(t)-\boldsymbol{k}_{x i}^{*}$, and $\tilde{k}_{r i}(t)=k_{r i}(t)-k_{r i}^{*}$, we can obtain the closed-loop system

$$
\begin{aligned}
& \dot{\boldsymbol{x}}=\sum_{i=1}^{l} \boldsymbol{A}_{m i} \chi_{i} \boldsymbol{x}+\sum_{i=1}^{l} \boldsymbol{b}_{m i} \chi_{i} r+\sum_{i=1}^{l} \boldsymbol{b}_{m i} \chi_{i} \sum_{i=1}^{l} \frac{1}{k_{r i}^{*}}\left(\tilde{\boldsymbol{k}}_{x i}^{\boldsymbol{\top}} \chi_{i} \boldsymbol{x}+\tilde{k}_{r i} \chi_{i} r\right) \\
& y=\boldsymbol{c}^{\boldsymbol{\top}} \boldsymbol{x}
\end{aligned}
$$

In view of (6.1.4) and (6.1.9), the tracking error equation follows:

$$
e(t)=W_{m}(s)\left[\sum_{i=1}^{l} \frac{1}{k_{r i}^{*}}\left(\tilde{\boldsymbol{k}}_{x i}^{\top} \chi_{i} \boldsymbol{x}+\tilde{k}_{r i} \chi_{i} r\right)\right](t)+\eta(t)+\eta_{0}(t)+\varepsilon_{0}(t)
$$

where $\eta_{0}(t)$ and $\varepsilon_{0}(t)$ are as in (6.1.12) and (6.1.11), respectively, and $\eta(t)$ is in the same form of $\eta_{0}(t)$ :

$$
\begin{aligned}
\eta(t) & =\sum_{j=2}^{k} \int_{t_{j-2}}^{t_{j-1}}\left[\boldsymbol{c}^{\top} \boldsymbol{\Phi}(t, \tau) \boldsymbol{b}_{m(j-2)}-\boldsymbol{c}_{m}^{\top} \boldsymbol{\Phi}_{m}(t, \tau) \boldsymbol{b}_{m}\right] \Delta(\tau) d \tau \\
\Delta(\tau) & =\frac{1}{k_{r(j-2)}^{*}}\left(\tilde{\boldsymbol{k}}_{x(j-2)}^{\top}(\tau) \boldsymbol{x}(\tau)+\tilde{k}_{r(j-2)}(\tau) r(\tau)\right)
\end{aligned}
$$

with $(j-2)$ in the subscript denoting the active subsystem over $\left[t_{j-2}, t_{j-1}\right)$.

Remark 6.1.5. Similar to $\eta_{0}(t), \eta(t)$ captures the unparameterized part of the output tracking error $e(t)$ due to controller parameter errors, in the presence of subsystem switches. They are treated as perturbations to the parameterizable part of e $(t)$ in subsequent analysis. A property of $\eta(t)$ is that with the controller parameter estimates generated by parameter projection adaptive laws (developed next), a normalized version of $\eta(t)$ is small in the mean square sense, which is critical to closed-loop system stability analysis. 
Error Model. To derive an estimation error model for adaptive law design, we denote $\boldsymbol{\theta}_{i}(t)=\left[\boldsymbol{k}_{x i}^{\top}(t), k_{r i}(t)\right]^{\top}, \boldsymbol{\theta}_{i}^{*}=\left[\boldsymbol{k}_{x i}^{* \boldsymbol{\top}}, k_{r i}^{*}\right]^{\top}$, and define the auxiliary signals $\boldsymbol{\omega}(t)=\left[\boldsymbol{x}^{\top}(t), r(t)\right]^{\boldsymbol{\top}}, \boldsymbol{\zeta}_{i}(t)=W_{m}(s)\left[\boldsymbol{\omega} \chi_{i}\right](t), \xi_{i}(t)=\boldsymbol{\theta}_{i}^{\top}(t) \boldsymbol{\zeta}_{i}(t)-W_{m}(s)\left[\boldsymbol{\theta}_{i}^{\top} \boldsymbol{\omega} \chi_{i}\right](t)$ and the estimation error signal

$$
\epsilon(t)=e(t)+\sum_{i=1}^{l} \rho_{i}(t) \xi_{i}(t)
$$

where $\rho_{i}(t)$ is an estimate of $\rho_{i}^{*}=1 / k_{r i}^{*}$. With (6.1.17) in (6.1.20) we have the estimation error model

$$
\epsilon(t)=\sum_{i=1}^{l}\left(\rho_{i}^{*} \tilde{\boldsymbol{\theta}}_{i}^{\top}(t) \boldsymbol{\zeta}_{i}(t)+\tilde{\rho}_{i}(t) \xi_{i}(t)\right)+\eta(t)+\eta_{0}(t)+\varepsilon_{0}(t)
$$

where the parameterized part of $\epsilon(t)$ is linear in parameter errors $\tilde{\boldsymbol{\theta}}_{i}(t)=\boldsymbol{\theta}_{i}(t)-\boldsymbol{\theta}_{i}^{*}$, $\tilde{\rho}_{i}(t)=\rho_{i}(t)-\rho_{i}^{*}$. For the rest part of $\varepsilon(t), \eta(t)+\eta_{0}(t)$ is due to the presence of subsystem switches, while $\epsilon_{0}(t)$ is related to system initial conditions. They are not parameterizable and are thus treated as perturbations. Parameter projection adaptive laws are proposed next, which are robust to such perturbations under additional slow switching conditions.

Adaptive Laws. Based on the error model (6.1.21), we propose the following gradient adaptive laws with parameter projections to update $\boldsymbol{\theta}_{i}(t)$ and $\rho_{i}(t), i \in \mathcal{I}$ :

$$
\begin{aligned}
& \dot{\boldsymbol{\theta}}_{i}(t)=-\frac{\operatorname{sign}\left[k_{p i}\right] \boldsymbol{\Gamma}_{i} \boldsymbol{\zeta}_{i}(t) \epsilon(t)}{m^{2}(t)}+\boldsymbol{f}_{i}(t), \quad \boldsymbol{\Gamma}_{i}=\boldsymbol{\Gamma}_{i}^{\boldsymbol{\top}}>\mathbf{0} \\
& \dot{\rho}_{i}(t)=-\frac{\gamma_{i} \xi_{i}(t) \epsilon(t)}{m^{2}(t)}+g_{i}(t), \quad \gamma_{i}>0, \quad t \geq 0
\end{aligned}
$$

assuming certain knowledge of the bounds on the nominal controller parameters $\boldsymbol{\theta}_{i}^{*}$ and $\rho_{i}^{*}$, where $\boldsymbol{f}_{i}(t)$ and $g_{i}(t)$ are the projection terms so designed as to confine the parameter estimates within their respective known bounds at all time. The initial estimates $\boldsymbol{\theta}_{i}\left(t_{0}\right)=\boldsymbol{\theta}_{i 0}$ and $\rho_{i}\left(t_{0}\right)=\rho_{i 0}$ are chosen to be within these bounds. The 
adaptation gains are $\boldsymbol{\Gamma}_{i} \in \mathbb{R}^{(n+1) \times(n+1)}, \gamma_{i} \in \mathbb{R}$, and the normalizing signal is $m^{2}(t)=$ $1+m_{s}(t)$ with $m_{s}(t)$ generated from

$$
\dot{m}_{s}(t)=-2 \delta_{0} m_{s}(t)+u^{2}(t)+y^{2}(t), \quad m_{s}(0)=0
$$

where $\delta_{0}<\delta$ for $\delta$ in Assumption (A6.1-2).

Stability Properties. The closed-loop stability and tracking performance of the the proposed adaptive control scheme are summarized in the following theorem:

Theorem 6.1.6. Consider the closed-loop system consisting of the plant (6.1.1), the reference model (6.1.4), and the adaptive controller (6.1.15) updated by the adaptive laws (6.1.22). There exist $T_{0}^{*}>0$ such that for $T_{0}=\min _{k \in \mathbb{Z}^{+}}\left\{t_{k}-t_{k-1}\right\} \geq T_{0}^{*}$, all signals in the closed-loop system are bounded, and for some positive constants $C_{2}, K_{2}$, the output tracking error $e(t)=y(t)-y_{m}(t)$ satisfies

$$
\int_{t}^{t+T} e^{2}(\tau) d \tau \leq C_{2}+K_{2} n_{T}, \quad \forall t \geq t_{0}, \quad \forall T \geq 0
$$

where $n_{T}$ is the total number of subsystem switches over $[t, t+T]$.

For closed-loop stability, we first note that the parameter estimates from the adaptive laws (6.1.22) have the desired properties that they are bounded and within their respective parameter bounds, and the normalized estimation error $\epsilon(t) / m(t)$, $\dot{\boldsymbol{\theta}}(t)$, and $\dot{\rho}(t)$ are small in the mean square sense, as long as the truncated system input and output signals are bounded. In particular, consider the positive definite function

$$
V\left(\tilde{\boldsymbol{\theta}}_{i}, \tilde{\rho}_{i}\right)=\sum_{i=1}^{l}\left(\left|\rho_{i}^{*}\right| \tilde{\boldsymbol{\theta}}_{i}^{\top} \boldsymbol{\Gamma}_{i}^{-1} \tilde{\boldsymbol{\theta}}_{i}+\gamma_{i}^{-1} \tilde{\rho}_{i}^{2}\right)
$$

whose time derivative along the trajectories of (6.1.22) is

$$
\begin{aligned}
\dot{V} & =-\frac{\epsilon^{2}(t)}{m^{2}(t)}+2\left(\frac{\eta(t)+\eta_{0}(t)}{m(t)}\right)^{2}+2 \frac{\varepsilon_{0}^{2}(t)}{m^{2}(t)}+2 \sum_{i=1}^{l}\left(\left|\rho_{i}^{*}\right| \tilde{\boldsymbol{\theta}}_{i}^{\top} \boldsymbol{\Gamma}_{i}^{-1} \boldsymbol{f}_{i}(t)+\gamma_{i}^{-1} \tilde{\rho}_{i} g_{i}(t)\right) \\
& \leq-\frac{\epsilon^{2}(t)}{m^{2}(t)}+2\left(\frac{\eta(t)+\eta_{0}(t)}{m(t)}\right)^{2}+2 \frac{\varepsilon_{0}^{2}(t)}{m^{2}(t)}
\end{aligned}
$$


The last inequality is due to the fact that $\tilde{\boldsymbol{\theta}}_{i}^{\top} \boldsymbol{\Gamma}_{i}^{-1} \boldsymbol{f}_{i}(t)<0$ and $\tilde{\rho}_{i} g_{i}(t)<0$. It then follows that

$$
\int_{t}^{t+T} \frac{\epsilon^{2}(\tau)}{m^{2}(\tau)} d \tau \leq c_{1}+2 \int_{t}^{t+T}\left[\left(\frac{\eta(\tau)+\eta_{0}(\tau)}{m(\tau)}\right)^{2}+\frac{\varepsilon_{0}^{2}(\tau)}{m^{2}(\tau)}\right] d \tau
$$

for some $c_{1}>0$, and the same properties apply to $\dot{\boldsymbol{\theta}}(t)$ and $\dot{\rho}(t)$ as well.

Closed-loop signal boundedness can be proved by first using a reduced-order state observer design of the piecewise linear system (6.1.1) to parameterize the state feedback controller structure in (6.1.6) into an output feedback form. A fictitious signal is then defined in a similar way as $m(t)$, such that the various signals in the closed-loop systems when normalized by this fictitious signal are either bounded or small in the mean square sense. A filtered version of the system output $y(t)$ is then expressed in a feedback framework that is suitable for the application of the small gain theorem. Under sufficiently slow subsystem switches $\left(T_{0} \geq T_{0}^{*}\right.$ for some $\left.T_{0}^{*}>0\right)$ and with the signal properties as stated above, the boundedness of the fictitious signal can be concluded, and the closed-loop signal boundedness is established.

The mean square tracking performance can be obtained by first dividing the integration time interval $[t, t+T]$ into corresponding switching time intervals. The integral of $e^{2}(t) / m^{2}(t)$ over each interval is upper bounded by a sum of those of $\epsilon^{2}(t) / m^{2}(t)$, $\|\dot{\boldsymbol{\theta}}(t)\|$, and $\left|\dot{\rho}^{2}(t)\right|$. With their respective mean square properties, it can be shown for some $c_{2}, c_{3}>0$ that

$$
\int_{t}^{t+T} e^{2}(\tau) d \tau \leq c_{2}+c_{3} \int_{t}^{t+T}\left(\eta(\tau)+\eta_{0}(\tau)\right)^{2} d \tau
$$

Note that with sufficiently slow switches, $\varepsilon_{0}(t)$ is exponentially stable and thus belongs to $\mathcal{L}^{2}$. The tracking performance $(6.1 .24)$ can then be established from (6.1.26) as in Theorem 6.1.2, with the fact that $\eta(t)$ and $\eta_{0}(t)$ are small in the mean square sense. 
Remark 6.1.7. If the system ceases to switch after some time, $\eta(t)$ and $\eta_{0}(t)$ will decay to zero exponentially. We can conclude from (6.1.24) that $\lim _{t \rightarrow \infty} e(t)=0$ and $e(t) \in \mathcal{L}^{2}$.

\subsubsection{Recovery of Asymptotic Tracking Performance}

Conditions under which the asymptotic tracking performance is achieved with the proposed adaptive control scheme are of interest. The following theorem gives such a condition:

Theorem 6.1.8. Suppose there exist nominal controller parameters $\boldsymbol{k}_{x i}^{*} \in \mathbb{R}^{n}$ and $k_{r i}^{*} \in \mathbb{R}$, such that in addition to (6.1.8) and (6.1.9),

$$
\boldsymbol{A}_{m i}=\boldsymbol{A}_{m}^{*}, \quad \boldsymbol{b}_{m i}=\boldsymbol{b}_{m}^{*}, \quad i \in \mathcal{I}
$$

for some constant matrices $\boldsymbol{A}_{m}^{*} \in \mathbb{R}^{n \times n}$ and $\boldsymbol{b}_{m}^{*} \in \mathbb{R}^{n}$. Then all signals in the closed-loop system, consisting of the plant (6.1.1), the reference model (6.1.4), and the adaptive controller (6.1.15) updated by

$$
\begin{aligned}
& \dot{\boldsymbol{\theta}}_{i}(t)=-\frac{\operatorname{sign}\left[k_{p i}\right] \boldsymbol{\Gamma}_{i} \boldsymbol{\zeta}_{i}(t) \epsilon(t)}{m^{2}(t)}, \quad \boldsymbol{\Gamma}_{i}=\boldsymbol{\Gamma}_{i}^{\boldsymbol{\top}}>\mathbf{0} \\
& \dot{\rho}_{i}(t)=-\frac{\gamma_{i} \xi_{i}(t) \epsilon(t)}{m^{2}(t)} \gamma_{i}>0, \quad t \geq 0
\end{aligned}
$$

are bounded, and the output tracking error $e(t)=y(t)-y_{m}(t)$ satisfies $e(t) \in \mathcal{L}^{2}$ and converges to zero asymptotically, i.e., $\lim _{t \rightarrow \infty} e(t)=0$, for arbitrary subsystem switches.

Proof: Under (6.1.27), the matching condition (6.1.7) reduces to $\boldsymbol{c}^{\boldsymbol{\top}}\left(s \boldsymbol{I}-\boldsymbol{A}_{m}^{*}\right)^{-1} \boldsymbol{b}_{m}^{*}=$ $W_{m}(s)$, hence $\eta(t)=\eta_{0}(t)=0$ in (6.1.17), and $\varepsilon_{0}(t)$ is an initial condition related term that decays to zero exponentially. The estimation error $\epsilon(t)$ defined by $(6.1 .20)$ can thus be fully parameterized as in (6.1.21) with $\eta(t)=\eta_{0}(t)=0$, and no parameter projections are needed in the adaptive laws. 
Consider the positive definite function in (6.1.25). Its time derivative along the trajectories of $(6.1 .28)$ is $\dot{V}\left(\tilde{\boldsymbol{\theta}}_{i}, \tilde{\rho}_{i}\right) \leq-\epsilon^{2}(t) / m^{2}(t)$. Hence $\boldsymbol{\theta}_{i}(t), \rho_{i}(t) \in \mathcal{L}^{\infty}, \frac{\epsilon(t)}{m(t)} \in$ $\mathcal{L}^{2} \cap \mathcal{L}^{\infty}$. From (6.1.28) and $\frac{\zeta_{i}(t)}{m(t)}, \frac{\xi_{i}(t)}{m(t)} \in \mathcal{L}^{\infty}$, we have $\dot{\boldsymbol{\theta}}_{i}(t), \dot{\rho}_{i}(t) \in \mathcal{L}^{2} \cap \mathcal{L}^{\infty}$. With these desired signal properties, closed-loop stability and asymptotic tracking performance can be established by the standard stability analysis procedure, after using a reducedorder state observer design of (6.1.1) to parameterize the state feedback controller structure in (6.1.6) into an output feedback form [15].

Remark 6.1.9. Compared with (6.1.8) and (6.1.9), the condition of (6.1.27) is restrictive: each subsystem is required to be matched via the model reference controller (6.1.6) to the same linear time-invariant closed-loop system $\left(\boldsymbol{c}^{\top}, \boldsymbol{A}_{m}^{*}, \boldsymbol{b}_{m}^{*}\right)$. In practice, this may not be satisfied. However, systems in certain canonical forms fit in this context. A simulation example is shown in Section 6.3 for systems in controllable canonical forms.

\subsubsection{Advantages over Conventional MRAC}

If conventional SISO state feedback for output tracking MRAC design were to be applied to the piecewise linear system (6.1.1), the controller structure will be in the same form as (6.1.15), i.e.,

$$
u(t)=\boldsymbol{k}_{x}^{\top}(t) \boldsymbol{x}(t)+k_{r}(t) r(t)
$$

However, as estimates of the nominal controller parameters $\boldsymbol{k}_{x}^{*}(t)$ and $k_{r}^{*}(t)$ defined as in (6.1.6), $\boldsymbol{k}_{x}(t)$ and $k_{r}(t)$ are directly updated by some adaptive laws.

Since the nominal parameters $\boldsymbol{\theta}^{*}(t) \triangleq\left[\boldsymbol{k}_{x}^{\top *}(t), k_{r}^{*}(t)\right]^{\top}$ and $\rho^{*}(t) \triangleq 1 / k_{r}^{*}(t)$ are timevarying (piecewise constant), and only one set of parameters $\boldsymbol{\theta}(t) \triangleq\left[\boldsymbol{k}_{x}^{\top}(t), k_{r}(t)\right]^{\top}$ is updated for control implementation, additional modeling error will be introduced in 
the error model, compared with the proposed adaptive scheme. To be specific, the tracking error with the application of such a control law is

$$
e(t)=W_{m}(s)\left[\rho^{*} \tilde{\boldsymbol{\theta}}^{\boldsymbol{\top}} \boldsymbol{\omega}\right](t)+\eta(t)+\eta_{0}(t)+\varepsilon_{0}(t)
$$

where $\tilde{\boldsymbol{\theta}}(t)=\boldsymbol{\theta}(t)-\boldsymbol{\theta}^{*}(t), \eta_{0}(t)$ and $\varepsilon_{0}(t)$ are as in (6.1.12) and (6.1.11), and $\eta(t)$ is as in (6.1.18) with $\Delta(\tau)=\rho^{*}(\tau) \tilde{\boldsymbol{\theta}}^{\top}(\tau) \boldsymbol{\omega}(\tau)$. By defining the estimation error signal $\epsilon(t)=e(t)+\rho(t) \xi(t)$ with $\xi(t)=\boldsymbol{\theta}^{\top}(t) \boldsymbol{\zeta}(t)-W_{m}(s)\left[\boldsymbol{\theta}^{\top} \boldsymbol{\omega}\right](t)$ and $\boldsymbol{\zeta}(t)=W_{m}(s)[\boldsymbol{\omega}](t)$, it follows that

$$
\begin{aligned}
\epsilon(t)= & \rho^{*}(t) \tilde{\boldsymbol{\theta}}^{\top}(t) \boldsymbol{\zeta}(t)+\tilde{\rho}(t) \xi(t)+\varepsilon_{a}(t)+\eta(t)+\eta_{0}(t)+\varepsilon_{0}(t) \\
\varepsilon_{a}(t)= & W_{m}(s)\left[\rho^{*} \tilde{\boldsymbol{\theta}}^{\boldsymbol{\top}} \boldsymbol{\omega}\right](t)-\rho^{*}(t) W_{m}(s)\left[\tilde{\boldsymbol{\theta}}^{\top} \boldsymbol{\omega}\right](t) \\
& +\rho^{*}(t)\left(\boldsymbol{\theta}^{* \boldsymbol{\top}}(t) \boldsymbol{\zeta}(t)-W_{m}(s)\left[\boldsymbol{\theta}^{* \boldsymbol{\top}} \boldsymbol{\omega}\right](t)\right)
\end{aligned}
$$

With the knowledge of lower and upper bounds on system parameters, the following parameter projection adaptive laws can be applied to update $\boldsymbol{\theta}(t)$ and $\rho(t)$ :

$$
\begin{aligned}
& \dot{\boldsymbol{\theta}}(t)=-\frac{\operatorname{sign}\left[\rho^{*}(t)\right] \boldsymbol{\Gamma} \boldsymbol{\zeta}(t) \epsilon(t)}{m^{2}(t)}+\boldsymbol{f}(t), \quad \boldsymbol{\Gamma}=\boldsymbol{\Gamma}^{\boldsymbol{\top}}>\mathbf{0} \\
& \dot{\rho}(t)=-\frac{\gamma \xi(t) \epsilon(t)}{m^{2}(t)}+g(t), \quad \gamma>0, \quad t \geq 0
\end{aligned}
$$

where the projection terms $\boldsymbol{f}(t)$ and $g(t)$ are designed to confine the parameter estimates to be within their respective bounds at all time.

To implement this design, we need to assume that $\rho^{*}(t)$ has a known and constant $\operatorname{sign}$; that is, $\operatorname{sign}\left[k_{p i}\right]$ is known and constant. Then with sufficient slow subsystem switches, closed-loop stability can be established.

The advantages of the proposed adaptive control scheme in Section 6.1.3 over this conventional MRAC design are: i) it has reduced modeling errors in the error model (there is an extra $\varepsilon_{a}(t)$ term in (6.1.30), as compared with (6.1.21)); ii) asymptotic tracking performance is recovered, when all subsystems can be matched to an LTI 
system with the same transfer function as the reference model system (see Theorem 6.1.8), while this cannot be archived by the conventional MRAC design; and iii) $\operatorname{sign}\left[k_{p i}\right]$ of each system model is not required to be the same. The price paid, on the other hand, is the knowledge of the indicator functions $\chi_{i}(t), i \in \mathcal{I}$.

\subsection{Design for MIMO Piecewise Linear Systems}

In this section, the adaptive control scheme proposed in Section 6.2 is extended to multivariable MRAC schemes applicable to MIMO piecewise linear system. A direct state feedback MRAC scheme is developed based on the LDS decomposition of high frequency gain matrices of such systems.

\subsubsection{Problem Statement}

Consider an $M$-input, $M$-output piecewise linear system:

$$
\begin{gathered}
\dot{\boldsymbol{x}}(t)=\boldsymbol{A}(t) \boldsymbol{x}(t)+\boldsymbol{B}(t) \boldsymbol{u}(t) \\
\boldsymbol{y}(t)=\boldsymbol{C}^{\top} \boldsymbol{x}(t), \quad \boldsymbol{x}(0)=\boldsymbol{x}_{0}
\end{gathered}
$$

where $\boldsymbol{x}(t) \in \mathbb{R}^{n}$ is the state vector and is available for measurement, $\boldsymbol{u}(t) \in \mathbb{R}^{M}$ is the control input, $\boldsymbol{y}(t) \in \mathbb{R}^{M}$ is the controlled output, $\boldsymbol{A}(t) \in \mathbb{R}^{n \times n}$ and $\boldsymbol{B}(t) \in \mathbb{R}^{n \times M}$ are unknown time-varying system parameter matrices, and $C \in \mathbb{R}^{n \times M}$ is an unknown constant parameter matrix. Similar to the SISO case, the parameter matrices $\boldsymbol{A}(t), \boldsymbol{B}(t)$ vary in a piecewise linear pattern; that is, during different time periods, $(\boldsymbol{A}(t), \boldsymbol{B}(t))$ take on different values as specified by the parameter matrix sets $\left(\boldsymbol{A}_{i}, \boldsymbol{B}_{i}\right)$, where $\boldsymbol{A}_{i}, \boldsymbol{B}_{i}$ are unknown but constant parameter matrices, and $l$ is the total number of subsystems. The indicator functions are introduced to characterize such a time-varying behavior of the system.

Indicator Functions. The indicator functions $\chi_{i}(t)$ are assumed to be known 
and defined as

$$
\chi_{i}(t)= \begin{cases}1, & \text { if }(\boldsymbol{A}(t), \boldsymbol{B}(t))=\left(\boldsymbol{A}_{i}, \boldsymbol{B}_{i}\right) \\ 0, & \text { otherwise }\end{cases}
$$

The have the following properties

$$
\sum_{i=1}^{l} \chi_{i}(t)=1, \quad \chi_{j}(t) \chi_{k}(t)=0, \quad j \neq k
$$

The time-varying parameter matrices $\boldsymbol{A}(t), \boldsymbol{B}(t)$ can then be expressed as

$$
\boldsymbol{A}(t)=\sum_{i=1}^{l} \boldsymbol{A}_{i} \chi_{i}(t), \quad \boldsymbol{B}(t)=\sum_{i=1}^{l} \boldsymbol{B}_{i} \chi_{i}(t)
$$

Control Objective. The control objective is to develop a state feedback control law $\boldsymbol{u}(t)$ for the piecewise linear system (6.2.1) with parameter variations characterized as in (6.2.2) such that all the signals in the closed-loop system are bounded, and $\boldsymbol{y}(t)$ tracks a reference signal $\boldsymbol{y}_{m}(t)$ as close as possible. The reference trajectory $\boldsymbol{y}_{m}(t)$ is generated from a reference model system

$$
\boldsymbol{y}_{m}(t)=\boldsymbol{W}_{m}(s)[\boldsymbol{r}](t), \quad \boldsymbol{W}_{m}(s)=\boldsymbol{\xi}_{m}^{-1}(s)
$$

where $\boldsymbol{\xi}_{m}(s)$ is a common modified left interactor matrix for the transfer matrix of each subsystem, i.e.,

$$
\boldsymbol{G}_{i}(s)=\boldsymbol{C}^{\boldsymbol{\top}}\left(s \boldsymbol{I}-\boldsymbol{A}_{i}\right)^{-1} \boldsymbol{B}_{i}
$$

of the piecewise linear system (6.2.1), and $\boldsymbol{r}(t) \in \mathbb{R}^{M}$ is a bounded, piecewise continuous external reference input signal.

MRAC Assumptions. Suppose for the $i$ th subsystem the transfer matrix of the system is (6.2.4). To design an adaptive state feedback control law for output tracking, the following assumptions are made for $i \in \mathcal{I}$ :

(A6.2-1) $\left(\boldsymbol{C}^{\top}, \boldsymbol{A}_{i}, \boldsymbol{B}_{i}\right)$ is stabilizable and detectible; 
(A6.2-2) All zeros of $\boldsymbol{G}_{i}(s)$ are stable with their real parts less than $-\delta$ for some known $\delta>0$;

(A6.2-3) $\boldsymbol{G}_{i}(s)$ are strictly proper, have full rank and their modified left interactor matrix $\boldsymbol{\xi}_{m}(s)$ is known;

(A6.2-4) All leading principle minors $\Delta_{i j}, j=1,2, \ldots, M$, of the high frequency gain matrices, $\boldsymbol{K}_{p i}=\lim _{s \rightarrow \infty} \boldsymbol{\xi}_{m}(s) \boldsymbol{G}_{i}(s)$, are nonzero with known signs; and

(A6.2-5) The LDS decompositions of $\boldsymbol{K}_{p i}[73]$ are such that

$$
\boldsymbol{K}_{p i}=\boldsymbol{L}_{s} \boldsymbol{D}_{s i} \boldsymbol{S}_{i}
$$

where $\boldsymbol{L}_{s} \in \mathbb{R}^{M \times M}$ is unity lower triangular, $\boldsymbol{S}_{i} \in \mathbb{R}^{M \times M}$ are symmetric, positive definite, and

$$
\boldsymbol{D}_{s i}=\operatorname{diag}\left\{\operatorname{sign}\left[\Delta_{i 1}\right] \gamma_{i 1}, \ldots, \operatorname{sign}\left[\frac{\Delta_{i M}}{\Delta_{i M-1}}\right] \gamma_{i M}\right\}
$$

with $\gamma_{i j}>0, j=1,2, \ldots, M$.

Remark 6.2.1. The assumed knowledge of $\delta$ in Assumption (A6.2-2) is for the design of a suitable dynamic normalizing signal, such that the boundedness property of the normalized system state vector can be used in concluding the closed-loop stability properties [11].

Under Assumption (A6.2-4), a gain matrix $\boldsymbol{K}_{p} \in \mathbb{R}^{M \times M}$ with nonzero leading principle minors $\Delta_{j}, j=1,2, \ldots, M$, has a unique LDU decomposition [15,73] (see Section 2.2), i.e., $\boldsymbol{K}_{p}=\boldsymbol{L} \boldsymbol{D}^{*} \boldsymbol{U}$, where $\boldsymbol{L}$ is unity lower triangular, $\boldsymbol{U}$ is unity upper triangular, and $\boldsymbol{D}^{*}=\operatorname{diag}\left\{\Delta_{1}, \frac{\Delta_{2}}{\Delta_{1}}, \ldots, \frac{\Delta_{M}}{\Delta_{M-1}}\right\}$. Note that its LDS decomposition, $\boldsymbol{K}_{p}=\boldsymbol{L}_{s} \boldsymbol{D}_{s} \boldsymbol{S}$, is not unique in that it follows from the unique LDU decomposition with $\boldsymbol{L}_{s}=$ $\boldsymbol{L} \boldsymbol{D}_{s}\left(\boldsymbol{U}^{-1}\right)^{\top} \boldsymbol{D}_{s}^{-1}, \boldsymbol{S}=\boldsymbol{U}^{\top} \boldsymbol{D}_{s}^{-1} \boldsymbol{D}^{*} \boldsymbol{U}$, and $\boldsymbol{D}_{s}=\operatorname{diag}\left\{\operatorname{sign}\left[\Delta_{1}\right] \gamma_{1}, \ldots, \operatorname{sign}\left[\frac{\Delta_{M}}{\Delta_{M-1}}\right] \gamma_{M}\right\}$ whose diagonal elements $\gamma_{j}>0$ may be chosen arbitrarily. 
Remark 6.2.2. Assumption (A6.2-5), that is, the high frequency gain matrix $\boldsymbol{K}_{p i}$ of each subsystem is assumed to have a common $\boldsymbol{L}_{s}$ matrix in their LDS decompositions (6.2.5), is essential in the derivation of the error model for adaptive control design to avoid differentiation operations on the output tracking error signal $\boldsymbol{e}(t)$, which is undesirable in practical applications with the presence of noises in signals. This assumption is trivial and automatically satisfied for SISO case (see Section 6.1). For a set of high frequency gain matrices $\boldsymbol{K}_{p i} \in \mathbb{R}^{M \times M}$ with $M>1$, such $\boldsymbol{L}_{s}$ may exist for a specific choice of $\boldsymbol{D}_{s}$. This is shown by an illustrative example in Section 6.3.

\subsubsection{Nominal Control Scheme}

Controller Structure. If the system parameter matrices $\boldsymbol{A}_{i}$ and $\boldsymbol{B}_{i}, i \in \mathcal{I}$, are known, the following state feedback control law is applied

$$
\boldsymbol{u}(t)=\boldsymbol{K}_{x}^{* \top}(t) \boldsymbol{x}(t)+\boldsymbol{K}_{r}^{*}(t) \boldsymbol{r}(t)
$$

with the controller parameter matrices

$$
\begin{aligned}
& \boldsymbol{K}_{x}^{*}(t)=\boldsymbol{K}_{x 1}^{*} \chi_{1}(t)+\boldsymbol{K}_{x 2}^{*} \chi_{2}(t)+\cdots+\boldsymbol{K}_{x l}^{*} \chi_{l}(t) \\
& \boldsymbol{K}_{r}^{*}(t)=\boldsymbol{K}_{r 1}^{*} \chi_{1}(t)+\boldsymbol{K}_{r 2}^{*} \chi_{2}(t)+\cdots+\boldsymbol{K}_{r l}^{*} \chi_{l}(t)
\end{aligned}
$$

where $\boldsymbol{K}_{x i}^{*} \in \mathbb{R}^{n \times M}$ and $\boldsymbol{K}_{r i}^{*} \in \mathbb{R}^{M}$ are computed from the piecewise plant-model matching condition:

$$
\boldsymbol{C}^{\boldsymbol{\top}}\left(s \boldsymbol{I}-\boldsymbol{A}_{i}-\boldsymbol{B}_{i} \boldsymbol{K}_{x i}^{* \top}\right) \boldsymbol{B}_{i} \boldsymbol{K}_{r i}^{*}=\boldsymbol{W}_{m}(s), \quad \boldsymbol{K}_{r i}^{*-1}=\boldsymbol{K}_{p i}
$$

When the $i$ th subsystem is active (as indicated by $\chi_{i}(t)=1$ ), the controller (6.2.6) leads to an input/output transfer matrix matching of the closed-loop system $\left(\boldsymbol{C}^{\top}, \boldsymbol{A}_{m i}, \boldsymbol{B}_{m i}\right)$ with

$$
\boldsymbol{A}_{m i} \triangleq \boldsymbol{A}_{i}+\boldsymbol{B}_{i} \boldsymbol{K}_{x i}^{* \top}, \quad \boldsymbol{B}_{m i} \triangleq \boldsymbol{B}_{i} \boldsymbol{K}_{r i}^{*}
$$


to the reference model system, i.e.,

$$
\boldsymbol{C}^{\top}\left(s \boldsymbol{I}-\boldsymbol{A}_{m i}\right)^{-1} \boldsymbol{B}_{m i}=\boldsymbol{W}_{m}(s)
$$

The existence of $\boldsymbol{K}_{x i}^{*}, \boldsymbol{K}_{r i}^{*}$ is guaranteed by Assumption (Achap:sfot.2-1) and (Achap:sfot.22) [75]. Furthermore, the matrices $\boldsymbol{A}_{m i}$ are all stable.

With (6.2.6) in (6.2.1), the closed-loop system becomes

$$
\dot{\boldsymbol{x}}(t)=\sum_{i=1}^{l}\left(\boldsymbol{A}_{m i} \chi_{i} \boldsymbol{x}(t)+\boldsymbol{B}_{m i} \chi_{i} \boldsymbol{r}(t)\right), \quad \boldsymbol{y}(t)=\boldsymbol{C}^{\top} \boldsymbol{x}(t)
$$

Similar to the SISO case, with the results of Lemma 5.2.1, we can establish the stability properties of the nominal closed-loop system 6.2.10 as follows.

Stability Properties. Let $\left(\boldsymbol{C}^{\boldsymbol{\top}}, \boldsymbol{A}_{m(k-1)}, \boldsymbol{B}_{m(k-1)}\right) \in\left\{\left(\boldsymbol{C}^{\boldsymbol{\top}}, \boldsymbol{A}_{m i}, \boldsymbol{B}_{m i}\right)\right\}_{i=1}^{l}$ denote the active subsystem over $\left[t_{k-1}, t_{k}\right), k \in \mathbb{Z}^{+}, \boldsymbol{\Phi}(t, \tau)$ the state transition matrix associated with the homogeneous system of (6.2.10), and $\left(\boldsymbol{C}_{m}^{\top}, \boldsymbol{A}_{m}, \boldsymbol{B}_{m}\right)$ a state space realization of the reference model system (6.2.3) with the associated state transition matrix $\boldsymbol{\Phi}_{m}(t, \tau)$. For the nominal closed-loop system, consisting of the plant (6.2.1), the reference model (6.2.3), and the controller (6.2.6), we have the following results:

Theorem 6.2.3. All signals in the nominal closed-loop system are bounded if the minimum switching time interval $T_{0}$ satisfies (5.2.4). The output tracking error $e(t)=$ $y(t)-y_{m}(t)$ is

$$
\boldsymbol{e}(t)=\boldsymbol{\eta}_{0}(t)+\varepsilon_{0}(t)
$$

with $\varepsilon_{0}(t)$ an initial condition related term

$$
\boldsymbol{\varepsilon}_{0}(t)=\boldsymbol{C}^{\boldsymbol{\top}} \boldsymbol{\Phi}\left(t, t_{0}\right) \boldsymbol{x}\left(t_{0}\right)-\boldsymbol{C}_{m}^{\boldsymbol{\top}} \boldsymbol{\Phi}_{m}\left(t, t_{0}\right) \boldsymbol{x}_{m}\left(t_{0}\right)
$$

which is exponentially decaying, and for $t \in\left[t_{k-1}, t_{k}\right)$,

$$
\boldsymbol{\eta}_{0}(t)=\sum_{j=2}^{k} \int_{t_{j-2}}^{t_{j-1}}\left[\boldsymbol{C}^{\top} \boldsymbol{\Phi}(t, \tau) \boldsymbol{B}_{m(j-2)}-\boldsymbol{C}_{m}^{\top} \boldsymbol{\Phi}_{m}(t, \tau) \boldsymbol{B}_{m}\right] \boldsymbol{r}(\tau) d \tau
$$


which is small in the mean square sense, under the slow switching condition (5.2.4). Furthermore, there exist positive constants $C_{1}$ and $K_{1}$, such that $\boldsymbol{e}(t)$ satisfies

$$
\int_{t}^{t+T}\|e(\tau)\|^{2} d \tau \leq C_{1}+K_{1} n_{T}, \quad \forall t \geq t_{0}, \quad \forall T \geq 0
$$

where $n_{T}$ is the total number of subsystem switches over $[t, t+T]$.

Proof: The proof follows the same line as in the SISO case (see Section 6.1.2), thus the details are omitted.

Similar to the SISO case, the comments made in Remarks 6.1.3 and 6.1.4 also apply here. In particular, if the system stops switching after some time, the output tracking error decays to zero exponentially. Furthermore, $\boldsymbol{\eta}_{0}(t)$ in (6.2.12) captures the deviations of $\boldsymbol{y}(t)$ from $\boldsymbol{y}_{m}(t)$ caused by subsystem switches at $t_{k}, k \in \mathbb{Z}^{+}$.

\subsubsection{Adaptive Control Scheme}

Since the plant parameter matrices $\boldsymbol{A}_{i}$ and $\boldsymbol{B}_{i}, i \in \mathcal{I}$, are unknown, an adaptive control law with its parameters updated by some adaptive laws is needed.

Controller Structure. The adaptive version of (6.2.6) is proposed as follows:

$$
\boldsymbol{u}(t)=\boldsymbol{K}_{x}^{\top}(t) \boldsymbol{x}(t)+\boldsymbol{K}_{r}(t) \boldsymbol{r}(t)
$$

where $\boldsymbol{K}_{x}(t) \in \mathbb{R}^{n \times M}$ and $\boldsymbol{K}_{r}(t) \in \mathbb{R}^{M \times M}$ are defined as

$$
\boldsymbol{K}_{x}(t)=\sum_{i=1}^{l} \boldsymbol{K}_{x i}(t) \chi_{i}(t), \quad \boldsymbol{K}_{r}(t)=\sum_{i=1}^{l} \boldsymbol{K}_{r i}(t) \chi_{i}(t)
$$

The parameter matrices $\boldsymbol{K}_{x i}(t) \in \mathbb{R}^{n \times M}$ and $\boldsymbol{K}_{r i}(t) \in \mathbb{R}^{M \times M}$ are the adaptive estimates of $\boldsymbol{K}_{x i}^{*}$ and $\boldsymbol{K}_{r i}^{*}$, defined to satisfy the matching condition (6.2.7), and are updated from some adaptive laws to be developed as follows.

By applying the adaptive control law (6.2.14) to the plant (6.2.1), and defining

$$
\begin{gathered}
\tilde{\boldsymbol{K}}_{x}(t)=\boldsymbol{K}_{x}(t)-\boldsymbol{K}_{x}^{*}(t), \quad \tilde{\boldsymbol{K}}_{r}(t)=\boldsymbol{K}_{r}(t)-\boldsymbol{K}_{r}^{*}(t) \\
\tilde{\boldsymbol{K}}_{x i}(t)=\boldsymbol{K}_{x i}(t)-\boldsymbol{K}_{x i}^{*}, \quad \tilde{\boldsymbol{K}}_{r i}(t)=\boldsymbol{K}_{r i}(t)-\boldsymbol{K}_{r i}^{*}
\end{gathered}
$$


we obtain the closed-loop system

$$
\begin{aligned}
\dot{\boldsymbol{x}}(t) & =\sum_{i=1}^{l}\left(\boldsymbol{A}_{m i} \chi_{i}(t) \boldsymbol{x}(t)+\boldsymbol{B}_{m i} \chi_{i}(t) \boldsymbol{r}(t)\right) \\
& +\sum_{i=1}^{l} \boldsymbol{B}_{m i} \boldsymbol{K}_{r i}^{*-1}\left(\tilde{\boldsymbol{K}}_{x i}^{\top}(t) \chi_{i}(t) \boldsymbol{x}(t)+\tilde{\boldsymbol{K}}_{r i}(t) \chi_{i}(t) \boldsymbol{r}(t)\right) \\
\boldsymbol{y}(t) & =\boldsymbol{C}^{\top} \boldsymbol{x}(t)
\end{aligned}
$$

In view of (6.2.3) and (6.2.7), the tracking error equation is

$$
\begin{aligned}
\boldsymbol{e}(t) & =\boldsymbol{W}_{m}(s)\left[\sum_{i=1}^{l} \boldsymbol{K}_{p i} \tilde{\boldsymbol{\Theta}}_{i}^{\top} \chi_{i} \boldsymbol{\omega}\right](t)+\boldsymbol{\eta}(t)+\boldsymbol{\eta}_{0}(t)+\boldsymbol{\epsilon}_{0}(t) \\
\tilde{\boldsymbol{\Theta}}_{i}(t) & =\boldsymbol{\Theta}_{i}(t)-\boldsymbol{\Theta}_{i}^{*}(t), \quad \boldsymbol{\Theta}_{i}(t)=\left[\boldsymbol{K}_{x i}^{\top}(t), \boldsymbol{K}_{r i}(t)\right]^{\top} \\
\boldsymbol{\Theta}_{i}^{*} & =\left[\boldsymbol{K}_{x i}^{* \top}, \boldsymbol{K}_{r i}^{*}\right]^{\top}, \quad \boldsymbol{\omega}(t)=\left[\boldsymbol{x}^{\top}(t), \boldsymbol{r}(t)\right]^{\top}
\end{aligned}
$$

where $\boldsymbol{\eta}_{0}(t)$ and $\varepsilon_{0}(t)$ are as in (6.2.12) and (6.2.11), respectively, and $\boldsymbol{\eta}(t)$ is in the same form of $\boldsymbol{\eta}_{0}(t)$ :

$$
\begin{aligned}
\boldsymbol{\eta}(t) & =\sum_{j=2}^{k} \int_{t_{j-2}}^{t_{j-1}}\left[\boldsymbol{C}^{\boldsymbol{\top}} \boldsymbol{\Phi}(t, \tau) \boldsymbol{B}_{m(j-2)}-\boldsymbol{C}_{m}^{\boldsymbol{\top}} \boldsymbol{\Phi}_{m}(t, \tau) \boldsymbol{B}_{m}\right] \Delta(\tau) d \tau \\
\Delta(\tau) & =\boldsymbol{K}_{p(j-2)} \tilde{\boldsymbol{\Theta}}_{(j-2)}(\tau) \boldsymbol{\omega}(\tau)
\end{aligned}
$$

with $(j-2)$ in the subscript denoting the active subsystem over $\left[t_{j-2}, t_{j-1}\right)$.

Remark 6.2.4. Similar to $\boldsymbol{\eta}_{0}(t), \boldsymbol{\eta}(t)$ captures the unparameterized part of the output tracking error $\boldsymbol{e}(t)$ due to controller parameter errors, in the presence of subsystem switches. They are treated as perturbations to the parameterizable part of $\boldsymbol{e}(t)$ in subsequent analysis. A property of $\boldsymbol{\eta}(t)$ is that with the controller parameter estimates generated by parameter projection adaptive laws (developed next), a normalized version of $\boldsymbol{\eta}(t)$ is small in the mean square sense, which is critical to closed-loop system stability analysis.

Error Model. With (6.2.3) and (6.2.16), it follows that

$$
\boldsymbol{\xi}_{m}(s)[\boldsymbol{e}](t)=\sum_{i=1}^{l} \boldsymbol{K}_{p i} \tilde{\boldsymbol{\Theta}}_{i}^{\top}(t) \chi_{i}(t) \boldsymbol{\omega}(t)+\boldsymbol{\xi}_{m}(s)\left[\boldsymbol{\eta}+\boldsymbol{\eta}_{0}+\boldsymbol{\epsilon}_{0}\right](t)
$$


To deal with the parametric uncertainties in $\boldsymbol{K}_{p i}$, their LDS decompositions are used under Assumption (A6.2-5). With (6.2.5) substituted into (6.2.18), we have

$$
\boldsymbol{L}_{s}^{-1} \boldsymbol{\xi}_{m}(s)[\boldsymbol{e}](t)=\sum_{i=1}^{l} \boldsymbol{D}_{s i} \boldsymbol{S}_{i} \tilde{\boldsymbol{\Theta}}_{i}^{\top}(t) \chi_{i}(t) \boldsymbol{\omega}(t)+\boldsymbol{L}_{s}^{-1} \boldsymbol{\xi}_{m}(s)\left[\boldsymbol{\eta}+\boldsymbol{\eta}_{0}+\boldsymbol{\epsilon}_{0}\right](t)
$$

By introducing $\Theta_{0}^{*}=\boldsymbol{L}_{s}^{-1}-\boldsymbol{I}$, which is lower triangular with zero diagonal elements, we obtain

$$
\boldsymbol{\Theta}_{0}^{*} \boldsymbol{\xi}_{m}(s)[\boldsymbol{e}](t)+\boldsymbol{\xi}_{m}(s)[\boldsymbol{e}](t)=\sum_{i=1}^{l} \boldsymbol{D}_{s i} \boldsymbol{S}_{i} \tilde{\boldsymbol{\Theta}}_{i}^{\top}(t) \chi_{i}(t) \boldsymbol{\omega}(t)+\boldsymbol{L}_{s}^{-1} \boldsymbol{\xi}_{m}(s)\left[\boldsymbol{\eta}+\boldsymbol{\eta}_{0}+\boldsymbol{\epsilon}_{0}\right](t)
$$

Operating both sides of (6.2.20) by $h(s) \boldsymbol{I}$, where $h(s)=1 / f(s)$ with $f(s)$ a monic stable polynomial of degree equal to the maximum degree of $\boldsymbol{\xi}_{m}(s)$, leads to

$$
\left[0, \boldsymbol{\theta}_{2}^{* \top} \boldsymbol{\eta}_{2}(t), \boldsymbol{\theta}_{3}^{* \top} \boldsymbol{\eta}_{3}(t), \ldots, \boldsymbol{\theta}_{M}^{* \top} \boldsymbol{\eta}_{M}(t)\right]^{\top}+\overline{\boldsymbol{e}}(t)=\sum_{i=1}^{l} \boldsymbol{D}_{s i} \boldsymbol{S}_{i} h(s)\left[\tilde{\boldsymbol{\Theta}}_{i}^{\top} \chi_{i} \boldsymbol{\omega}\right](t)+\boldsymbol{d}(t)
$$

where $\boldsymbol{\theta}_{j}^{*} \in \mathbb{R}^{j-1}, j=2, \ldots, M$, denotes the $j$ th row elements (non-zero part) of $\boldsymbol{\Theta}_{0}^{*}$,

$$
\begin{aligned}
\overline{\boldsymbol{e}}(t) & =\boldsymbol{\xi}_{m}(s) h(s)[\boldsymbol{e}](t)=\left[\bar{e}_{1}, \bar{e}_{2} \ldots, \bar{e}_{M}\right]^{\top}, \quad \boldsymbol{\eta}_{j}(t)=\left[\bar{e}_{1}, \bar{e}_{2} \ldots, \bar{e}_{j-1}\right]^{\top} \in \mathbb{R}^{j-1} \\
\boldsymbol{d}(t) & =\boldsymbol{L}_{s}^{-1} \boldsymbol{\xi}_{m}(s) h(s)\left[\boldsymbol{\eta}+\boldsymbol{\eta}_{0}+\boldsymbol{\epsilon}_{0}\right](t)
\end{aligned}
$$

Define the estimation error signal

$$
\boldsymbol{\epsilon}(t)=\left[0, \boldsymbol{\theta}_{2}^{\top}(t) \boldsymbol{\eta}_{2}(t), \boldsymbol{\theta}_{3}^{\top}(t) \boldsymbol{\eta}_{3}(t), \ldots, \boldsymbol{\theta}_{M}^{\top}(t) \boldsymbol{\eta}_{M}(t)\right]^{\top}+\overline{\boldsymbol{e}}(t)+\sum_{i=1}^{l} \boldsymbol{\Psi}_{i}(t) \boldsymbol{\xi}_{i}(t)
$$

where $\boldsymbol{\theta}_{j}(t), j=2,3, \ldots, M$, are the estimates of $\boldsymbol{\theta}_{j}^{*}$, and $\boldsymbol{\Psi}_{i}(t)$ are the estimates of $\boldsymbol{\Psi}_{i}^{*}=\boldsymbol{D}_{s i} \boldsymbol{S}_{i}$, and

$$
\boldsymbol{\xi}_{i}(t)=\boldsymbol{\Theta}_{i}^{\top}(t) \boldsymbol{\zeta}_{i}(t)-h(s)\left[\boldsymbol{\Theta}_{i}^{\top} \chi_{i} \boldsymbol{\omega}\right](t), \quad \boldsymbol{\zeta}_{i}(t)=h(s)\left[\chi_{i} \boldsymbol{\omega}\right](t)
$$

Based on (6.2.21), we can obtain the following error model:

$$
\begin{aligned}
\boldsymbol{\epsilon}(t)= & {\left[0, \tilde{\boldsymbol{\theta}}_{2}^{\top}(t) \boldsymbol{\eta}_{2}(t), \tilde{\boldsymbol{\theta}}_{3}^{\top}(t) \boldsymbol{\eta}_{3}(t), \ldots, \tilde{\boldsymbol{\theta}}_{M}^{\top}(t) \boldsymbol{\eta}_{M}(t)\right]^{\top} } \\
& +\sum_{i=1}^{l}\left(\tilde{\boldsymbol{\Psi}}_{i}(t) \boldsymbol{\xi}_{i}(t)+\boldsymbol{D}_{s i} \boldsymbol{S}_{i} \tilde{\boldsymbol{\Theta}}_{i}^{\top}(t) \boldsymbol{\zeta}_{i}(t)\right)+\boldsymbol{d}(t)
\end{aligned}
$$


where $\tilde{\boldsymbol{\theta}}_{j}(t)=\boldsymbol{\theta}_{j}(t)-\boldsymbol{\theta}_{j}^{*}, \tilde{\boldsymbol{\Psi}}_{i}(t)=\boldsymbol{\Psi}_{i}(t)-\boldsymbol{\Psi}_{i}^{*}(t)$ are the parameter errors. The unparameterized part of $\boldsymbol{\epsilon}(t), \boldsymbol{d}(t)$, is due to the presence of subsystem switches and initial conditions, and is treated as a perturbation. Parameter projection adaptive laws are proposed next, which are robust to such a perturbation under additional slow switching conditions.

Adaptive Laws. Based on the error model (6.2.23), we propose the following gradient adaptive laws to update $\boldsymbol{\theta}_{j}(t), \boldsymbol{\Theta}_{i}(t)$, and $\boldsymbol{\Psi}_{i}(t), i \in \mathcal{I}, j=2,3, \ldots, M$ :

$$
\begin{aligned}
\dot{\boldsymbol{\theta}}_{j}(t) & =-\frac{\boldsymbol{\Gamma}_{\boldsymbol{\theta}_{j}} \boldsymbol{\eta}_{j}(t) \epsilon_{j}(t)}{m^{2}(t)}+\boldsymbol{f}_{j}(t) \\
\dot{\boldsymbol{\Theta}}_{i}^{\top}(t) & =-\frac{\boldsymbol{D}_{s i} \boldsymbol{\epsilon}(t) \boldsymbol{\zeta}_{i}^{\top}(t)}{m^{2}(t)}+\boldsymbol{F}_{i}(t) \\
\dot{\boldsymbol{\Psi}}_{i}(t) & =-\frac{\boldsymbol{\Gamma}_{i} \boldsymbol{\epsilon}(t) \boldsymbol{\xi}_{i}^{\top}(t)}{m^{2}(t)}+\boldsymbol{H}_{i}(t)
\end{aligned}
$$

assuming certain knowledge of the bounds on the nominal controller parameters, where $\boldsymbol{\epsilon}(t)=\left[\epsilon_{1}(t), \epsilon_{2}(t), \ldots, \epsilon_{M}(t)\right]^{\top}, \boldsymbol{f}_{j}(t), \boldsymbol{F}_{i}(t)$, and $\boldsymbol{H}_{i}(t)$ are the projection terms so designed as to confine the parameter estimates within their respective known bounds at all time. The initial parameter estimates are chosen to be within these bounds. The adaptation gain matrices $\boldsymbol{\Gamma}_{\theta j}$ and $\boldsymbol{\Gamma}_{i}$ are positive definite and diagonal, and the normalizing signal is $m^{2}(t)=1+m_{s}(t)$ with $m_{s}(t)$ generated from

$$
\dot{m}_{s}(t)=-2 \delta_{0} m_{s}(t)+\|\boldsymbol{u}(t)\|^{2}+\|\boldsymbol{y}(t)\|^{2}, m_{s}(0)=0, \delta_{0}<\delta
$$

where $\delta_{0}<\delta$ for $\delta$ in Assumption (A6.2-2).

Along the line of derivations in this section, LDU and SDU decomposition based designs can be developed for adaptive state feedback control of piecewise linear systems for output tracking. Similar to Assumption (A6.2-5), the controlled subsystems in these designs need to share certain common structural characteristics (a common $\boldsymbol{L}$ matrix in the LDU based design, or a common $\boldsymbol{S}$ matrix in the SDU based design). 
Stability Properties. With the adaptive controller (6.2.14), updated by (6.2.24), applied to the plant (6.2.1), we have the closed-loop stability properties and tracking performance as stated in the following theorem:

Theorem 6.2.5. Consider the closed-loop system consisting of the plant (6.2.1), the reference model (6.2.3), and the adaptive controller (6.2.14) updated by the adaptive laws (6.2.24). There exist $T_{0}^{*}>0$ such that for $T_{0}=\min _{k \in \mathbb{Z}^{+}}\left\{t_{k}-t_{k-1}\right\} \geq T_{0}^{*}$, all closed-loop signals are bounded, and for some positive constants $C_{2}$ and $K_{2}$, the output tracking error $\boldsymbol{e}(t)=\boldsymbol{y}(t)-\boldsymbol{y}_{m}(t)$ satisfies

$$
\int_{t}^{t+T}\|\boldsymbol{e}(\tau)\|^{2} d \tau \leq C_{2}+K_{2} n_{T}, \quad \forall t \geq t_{0}, \quad \forall T \geq 0
$$

where $n_{T}$ is the total number of subsystem switches over $[t, t+T]$.

For closed-loop stability, we first note that the parameter estimates from the adaptive laws of (6.2.24) have the desired properties that they are bounded and within their respective parameter bounds. By considering the positive definite function

$$
V=\frac{1}{2} \sum_{j=2}^{M} \tilde{\boldsymbol{\theta}}_{j}^{\top} \boldsymbol{\Gamma}_{\theta_{j}}^{-1} \tilde{\boldsymbol{\theta}}_{j}+\sum_{i=1}^{l}\left(\operatorname{tr}\left[\tilde{\mathbf{\Psi}}_{i}^{\top} \boldsymbol{\Gamma}_{i}^{-1} \tilde{\boldsymbol{\Psi}}_{i}\right]+\operatorname{tr}\left[\tilde{\boldsymbol{\Theta}}_{i} \boldsymbol{S}_{i} \tilde{\boldsymbol{\Theta}}_{i}^{\top}\right]\right)
$$

and its time derivative along (6.2.24), together with the fact that $\tilde{\boldsymbol{\theta}}_{j}^{\top} \boldsymbol{\Gamma}_{\theta_{j}}^{-1} \boldsymbol{f}_{j} \leq 0$, $\operatorname{tr}\left[\tilde{\boldsymbol{\Psi}}_{i}^{\top} \boldsymbol{\Gamma}_{i}^{-1} \boldsymbol{H}_{i}\right] \leq 0$, and $\operatorname{tr}\left[\tilde{\boldsymbol{\Theta}}_{i} \boldsymbol{S}_{i} \boldsymbol{F}_{i}\right] \leq 0$, we can obtain that for some $c, k>0$ and $\boldsymbol{z}(t)=\frac{\boldsymbol{\epsilon}(t)}{m(t)}, \dot{\boldsymbol{\theta}}_{j}(t), \dot{\boldsymbol{\Theta}}_{i}(t), \dot{\boldsymbol{\Psi}}_{i}(t)$

$$
\int_{t}^{t+T}\|\boldsymbol{z}(\tau)\|^{2} d \tau \leq c+k \int_{t}^{t+T} \frac{\|\boldsymbol{d}(\tau)\|^{2}}{m^{2}(\tau)} d \tau, \quad \forall t \geq t_{0}, \quad \forall T \geq 0
$$

Closed-loop signal boundedness can then be established following the same line of arguments as in Section 6.1.3 for SISO piecewise linear systems.

The mean square tracking performance can be obtained by first dividing the integration time interval $[t, t+T]$ into corresponding switching time intervals. The 
integral of $\|\boldsymbol{e}(t)\|^{2} / m^{2}(t)$ over each interval is upper bounded by a sum of those of $\|\boldsymbol{\epsilon}(t)\|^{2} / m^{2}(t),\left\|\dot{\boldsymbol{\theta}}_{j}(t)\right\|,\left\|\dot{\boldsymbol{\Theta}}_{i}(t)\right\|$, and $\left\|\dot{\boldsymbol{\Psi}}_{i}(t)\right\|$. With their respective mean square properties, it can be shown that

$$
\int_{t}^{t+T}\|\boldsymbol{e}(\tau)\|^{2} d \tau \leq c_{2}+c_{3} \int_{t}^{t+T}\|\boldsymbol{d}(\tau)\|^{2} d \tau
$$

for some $c_{2}, c_{3}>0$. Note that with sufficiently slow subsystem switches, the tracking performance (6.2.26) can be established from (6.2.28) as in Theorem 6.1.6.

Remark 6.2.6. In the state feedback state tracking design for piecewise linear systems (Sections 5.3-5.4), a plant-model matching condition in the form of

$$
\boldsymbol{A}_{i}+\boldsymbol{B}_{i} \boldsymbol{K}_{x i}^{* \top}=\boldsymbol{A}_{m i}, \quad \boldsymbol{B}_{i} \boldsymbol{K}_{r i}^{*}=\boldsymbol{B}_{m i}
$$

is crucial and certain structural information about the plant parameter matrices $\boldsymbol{A}_{i}$, $\boldsymbol{B}_{i}$ are needed for the specification of $\boldsymbol{A}_{m i}, \boldsymbol{B}_{m i}$ in the piecewise linear reference model system. In the output tracking case, such restrictive matching conditions are relaxed; in particular, the triple $\left(\boldsymbol{A}_{i}+\boldsymbol{B}_{i} \boldsymbol{K}_{x i}^{* \top}, \boldsymbol{B}_{i} \boldsymbol{K}_{r i}^{*}, \boldsymbol{C}^{\boldsymbol{\top}}\right)$ here is only a state space realization of the transfer matrix $\boldsymbol{W}_{m}(s)$, to ensure input-output, piecewise plantmodel matching, which can always be satisfied under the stated assumptions. In other words, the existence of the parameter matrices $\boldsymbol{K}_{x i}^{*}, \boldsymbol{K}_{r i}^{*}$ is guaranteed.

Remark 6.2.7. If the system ceases to switch after some time, $\boldsymbol{\eta}(t)$ and $\boldsymbol{\eta}_{0}(t)$ will decay to zero exponentially. We then conclude from (6.2.26) that $\lim _{t \rightarrow \infty} \boldsymbol{e}(t)=0$ and $\boldsymbol{e}(t) \in \mathcal{L}^{2}$.

\subsubsection{Recovery of Asymptotic Tracking Performance}

Similar to the results stated in Theorem 6.1.8, asymptotic output tracking performance is achieved with the proposed adaptive control design under the following condition: 
Theorem 6.2.8. Suppose there exist nominal controller parameters $\boldsymbol{K}_{x i}^{*} \in \mathbb{R}^{n \times M}$ and $\boldsymbol{K}_{r i}^{*} \in \mathbb{R}^{M \times M}$, such that in addition to (6.2.8) and (6.2.9),

$$
\boldsymbol{A}_{m i}=\boldsymbol{A}_{m}^{*}, \quad \boldsymbol{B}_{m i}=\boldsymbol{B}_{m}^{*}, \quad i \in \mathcal{I}
$$

for some constant matrices $\boldsymbol{A}_{m}^{*} \in \mathbb{R}^{n \times n}$ and $\boldsymbol{B}_{m}^{*} \in \mathbb{R}^{n \times M}$. Then all signals in the closed-loop system, consisting of the plant (6.2.1), the reference model (6.2.3), and the adaptive controller (6.2.14) updated by

$$
\begin{aligned}
\dot{\boldsymbol{\theta}}_{j}(t) & =-\frac{\boldsymbol{\Gamma}_{\boldsymbol{\theta}_{j}} \boldsymbol{\eta}_{j}(t) \epsilon_{j}(t)}{m^{2}(t)} \\
\dot{\boldsymbol{\Theta}}_{i}^{\top}(t) & =-\frac{\boldsymbol{D}_{s i} \boldsymbol{\epsilon}(t) \boldsymbol{\zeta}_{i}^{\top}(t)}{m^{2}(t)} \\
\dot{\boldsymbol{\Psi}}_{i}(t) & =-\frac{\boldsymbol{\Gamma}_{i} \boldsymbol{\epsilon}(t) \boldsymbol{\xi}_{i}^{\top}(t)}{m^{2}(t)}
\end{aligned}
$$

are bounded, and the output tracking error $\boldsymbol{e}(t)=\boldsymbol{y}(t)-\boldsymbol{y}_{m}(t)$ satisfies $\boldsymbol{e}(t) \in \mathcal{L}^{2}$ and converges to zero asymptotically, i.e., $\lim _{t \rightarrow \infty} \boldsymbol{e}(t)=\mathbf{0}$, for arbitrary subsystem switches.

Proof: Under (6.2.29), the matching condition (6.2.7) reduces to $\boldsymbol{C}^{\boldsymbol{\top}}\left(s \boldsymbol{I}-\boldsymbol{A}_{m}^{*}\right)^{-1} \boldsymbol{B}_{m}^{*}=$ $\boldsymbol{W}_{m}(s)$, hence $\boldsymbol{\eta}(t)=\boldsymbol{\eta}_{0}(t)=0$ in (6.2.16), and $\boldsymbol{\varepsilon}_{0}(t)$ is an initial condition related term that decays to zero exponentially. The estimation error $\boldsymbol{\epsilon}(t)$ defined by (6.2.22) can thus be completely parameterized as in (6.2.23) with $\boldsymbol{\eta}(t)=\boldsymbol{\eta}_{0}(t)=\mathbf{0}$, and no parameter projections are needed in the adaptive laws.

Consider the positive definite function in (6.2.27). Its time derivative along the 
trajectories of $(6.2 .30)$ is

$$
\begin{aligned}
\dot{V} & =\sum_{j=2}^{M} \tilde{\boldsymbol{\theta}}_{j}^{\top} \boldsymbol{\Gamma}_{\theta_{j}}^{-1} \dot{\boldsymbol{\theta}}_{j}+\sum_{i=1}^{l}\left(\operatorname{tr}\left[\tilde{\boldsymbol{\Psi}}_{i}^{\top} \boldsymbol{\Gamma}_{i}^{-1} \dot{\boldsymbol{\Psi}}_{i}\right]+\operatorname{tr}\left[\tilde{\boldsymbol{\Theta}}_{i} \boldsymbol{S}_{i} \dot{\boldsymbol{\Theta}}_{i}^{\top}\right]\right) \\
& =-\sum_{j=2}^{M} \frac{\tilde{\boldsymbol{\theta}}_{j}^{\top} \boldsymbol{\eta}_{j}(t) \epsilon_{j}(t)}{m^{2}(t)}-\sum_{i=1}^{l} \operatorname{tr}\left[\frac{\tilde{\boldsymbol{\Psi}}_{i}^{\top} \boldsymbol{\epsilon}(t) \boldsymbol{\xi}_{i}^{\top}(t)}{m^{2}(t)}\right]-\sum_{i=1}^{l} \operatorname{tr}\left[\frac{\tilde{\boldsymbol{\Theta}}_{i} \boldsymbol{S}_{i} \boldsymbol{D}_{s i} \boldsymbol{\epsilon}(t) \boldsymbol{\zeta}_{i}^{\top}(t)}{m^{2}(t)}\right] \\
& =-\sum_{j=2}^{M} \frac{\tilde{\boldsymbol{\theta}}_{j}^{\top} \boldsymbol{\eta}_{j}(t) \epsilon_{j}(t)}{m^{2}(t)}-\sum_{i=1}^{l} \frac{\boldsymbol{\xi}_{i}^{\top}(t) \tilde{\boldsymbol{\Psi}}_{i}^{\top} \boldsymbol{\epsilon}(t)}{m^{2}(t)}-\sum_{i=1}^{l} \frac{\boldsymbol{\zeta}_{i}^{\top}(t) \tilde{\boldsymbol{\Theta}}_{i} \boldsymbol{S}_{i} \boldsymbol{D}_{s i} \boldsymbol{\epsilon}(t)}{m^{2}(t)} \\
& \leq-\frac{\boldsymbol{\epsilon}^{\top}(t) \boldsymbol{\epsilon}(t)}{m^{2}(t)} \leq \mathbf{0}
\end{aligned}
$$

This implies that $\boldsymbol{\theta}_{j}(t), \boldsymbol{\Theta}_{i}(t), \boldsymbol{\Psi}_{i}(t) \in \mathcal{L}^{\infty}, \frac{\boldsymbol{\epsilon}(t)}{m(t)} \in \mathcal{L}^{2} \cap \mathcal{L}^{\infty}$, and from (6.2.24) and the boundedness of $\frac{\boldsymbol{\eta}_{j}(t)}{m(t)}, \frac{\boldsymbol{\zeta}_{i}(t)}{m(t)}, \frac{\boldsymbol{\xi}_{i}(t)}{m(t)}$, we also have $\dot{\boldsymbol{\theta}}_{j}(t), \dot{\boldsymbol{\Theta}}_{i}(t), \dot{\boldsymbol{\Psi}}_{i}(t) \in \mathcal{L}^{2} \cap \mathcal{L}^{\infty}$. With these desired signal properties, closed-loop stability and asymptotic tracking performance can be established by the standard stability analysis procedure, after using a reducedorder state observer design of (6.2.1) to parameterize the state feedback controller structure in (6.2.6) into an output feedback form [15].

\subsubsection{Advantages over Conventional MRAC}

If conventional MIMO state feedback for output tracking MRAC design were to be applied to the piecewise linear system (6.2.1), the following controller structure (in the same form as (6.2.14)) is used

$$
\boldsymbol{u}(t)=\boldsymbol{K}_{x}^{\top}(t) \boldsymbol{x}(t)+\boldsymbol{K}_{r}(t) \boldsymbol{r}(t)
$$

where $\boldsymbol{K}_{x}(t)$ is an estimate of $\boldsymbol{K}_{x}^{*}(t)=\sum_{i=1}^{l} \boldsymbol{K}_{x i}^{*} \chi_{i}(t)$, and $\boldsymbol{K}_{r}(t)$ is an estimate of $\boldsymbol{K}_{r}^{*}(t)=\sum_{i=1}^{l} K_{r i}^{*} \chi_{i}(t)$. They are directly updated by some adaptive laws.

The tracking error with the application of such a control law is

$$
\boldsymbol{e}(t)=\boldsymbol{W}_{m}(s)\left[\boldsymbol{K}_{p} \tilde{\boldsymbol{\Theta}}^{\top} \boldsymbol{\omega}\right](t)+\boldsymbol{\eta}(t)+\boldsymbol{\eta}_{0}(t)+\boldsymbol{\varepsilon}_{0}(t)
$$


where $\boldsymbol{K}_{p}(t)=\sum_{i=1}^{l} \boldsymbol{K}_{r i}^{*-1} \chi_{i}(t), \boldsymbol{\omega}(t)=\left[\boldsymbol{x}^{\top}(t), \boldsymbol{r}(t)\right]^{\top}, \tilde{\boldsymbol{\Theta}}(t)=\boldsymbol{\Theta}(t)-\boldsymbol{\Theta}^{*}(t), \boldsymbol{\Theta}(t)=$ $\left[\boldsymbol{K}_{x}^{\top}(t), \boldsymbol{K}_{r}(t)\right]^{\top}, \boldsymbol{\Theta}^{*}(t)=\left[\boldsymbol{K}_{x}^{* \top}(t), \boldsymbol{K}_{r}^{*}(t)\right]^{\top}, \boldsymbol{\eta}_{0}(t)$ and $\boldsymbol{\varepsilon}_{0}(t)$ are as in (6.2.12) and (6.2.11), and $\boldsymbol{\eta}(t)$ is as in (6.2.17) with $\Delta(\tau)=\boldsymbol{K}_{p}(\tau) \tilde{\boldsymbol{\Theta}}(\tau) \boldsymbol{\omega}(\tau)$.

By defining the estimation error signal

$$
\boldsymbol{\epsilon}(t)=\left[0, \boldsymbol{\theta}_{2}^{\top}(t) \boldsymbol{\eta}_{2}(t), \ldots, \boldsymbol{\theta}_{M}^{\top}(t) \boldsymbol{\eta}_{M}(t)\right]^{\top}+\overline{\boldsymbol{e}}(t)+\boldsymbol{\Psi}(t) \boldsymbol{\xi}(t)
$$

where $\boldsymbol{\theta}_{j}(t), j=2,3, \ldots, M$, are the estimates of $\boldsymbol{\theta}_{j}^{*}$, and $\boldsymbol{\Psi}(t)$ is the estimate of $\boldsymbol{\Psi}^{*}(t)=\boldsymbol{L}_{s}^{-1} \boldsymbol{K}_{p}(t)$, and

$$
\begin{aligned}
& \boldsymbol{\xi}(t)=\boldsymbol{\Theta}^{\top}(t) \boldsymbol{\zeta}(t)-h(s)\left[\boldsymbol{\Theta}^{\top} \boldsymbol{\omega}\right](t), \quad \boldsymbol{\zeta}(t)=h(s)[\boldsymbol{\omega}](t) \\
& \overline{\boldsymbol{e}}(t)=\boldsymbol{\xi}_{m}(s) h(s)[\boldsymbol{e}](t)=\left[\bar{e}_{1}, \bar{e}_{2} \ldots, \bar{e}_{M}\right]^{\boldsymbol{\top}}, \quad \boldsymbol{\eta}_{j}(t)=\left[\bar{e}_{1}, \bar{e}_{2} \ldots, \bar{e}_{j-1}\right]^{\boldsymbol{\top}}
\end{aligned}
$$

An estimation error model can be obtained as

$$
\boldsymbol{\epsilon}(t)=\left[0, \tilde{\boldsymbol{\theta}}_{2}^{\top}(t) \boldsymbol{\eta}_{2}(t), \ldots, \tilde{\boldsymbol{\theta}}_{M}^{\top}(t) \boldsymbol{\eta}_{M}(t)\right]^{\top}+\tilde{\boldsymbol{\Psi}}(t) \boldsymbol{\xi}(t)+\boldsymbol{\Psi}^{*}(t) \tilde{\boldsymbol{\Theta}}^{\top}(t) \boldsymbol{\zeta}(t)+\boldsymbol{\varepsilon}_{s}(t)+\boldsymbol{d}(t)
$$

where $\varepsilon_{s}(t)$ is a swapping term due to parameter discontinuities:

$$
\boldsymbol{\varepsilon}_{s}(t)=h(s)\left[\boldsymbol{\Psi}^{*} \tilde{\boldsymbol{\Theta}} \boldsymbol{\omega}\right](t)-\boldsymbol{\Psi}^{*}(t) h(s)[\tilde{\boldsymbol{\Theta}} \boldsymbol{\omega}](t)+\boldsymbol{\Psi}^{*}(t)\left(\boldsymbol{\Theta}^{*}(t) \boldsymbol{\zeta}(t)-h(s)\left[\boldsymbol{\Theta}^{*} \boldsymbol{\omega}\right](t)\right)
$$

With the knowledge of lower and upper bounds on system parameters, the following parameter projection adaptive laws can be applied to update the controller parameters:

$$
\begin{aligned}
\dot{\boldsymbol{\theta}}_{j}(t) & =-\frac{\boldsymbol{\Gamma}_{\theta_{j}} \boldsymbol{\eta}_{j}(t) \epsilon_{j}(t)}{m^{2}(t)}+\boldsymbol{f}_{j}(t) \\
\dot{\boldsymbol{\Theta}}^{\top}(t) & =-\frac{\boldsymbol{D}_{s} \boldsymbol{\epsilon}(t) \boldsymbol{\zeta}^{\top}(t)}{m^{2}(t)}+\boldsymbol{F}(t) \\
\dot{\boldsymbol{\Psi}}(t) & =-\frac{\boldsymbol{\Gamma} \boldsymbol{\epsilon}(t) \boldsymbol{\xi}^{\top}(t)}{m^{2}(t)}+\boldsymbol{H}(t)
\end{aligned}
$$

where $\boldsymbol{\epsilon}(t)=\left[\epsilon_{1}(t), \ldots, \epsilon_{M}(t)\right]^{\top}, \boldsymbol{\Gamma}_{\theta_{j}}$ and $\boldsymbol{\Gamma}$ are diagonal, positive definite adaptation gain matrices, and $\boldsymbol{f}_{j}(t), \boldsymbol{F}(t), \boldsymbol{H}(t)$ are the projection terms designed to confine the parameter estimates to be within their respective bounds at all time. 
To implement this design, we need to assume for $\Delta_{i j}$, the leading principle minors of $\boldsymbol{K}_{p i}$, that

(A6.2-4.a) $\operatorname{sign}\left[\Delta_{i j}\right]=\operatorname{sign}\left[\Delta_{k j}\right], i, k \in \mathcal{I}, j=1,2, \ldots, M$

in addition to Assumption (A6.2-4). Then with sufficient slow subsystem switches, closed-loop stability can be established.

The advantages of the proposed adaptive control scheme over this conventional MRAC design are: i) it has reduced modeling errors in the error model (there is an extra term $\varepsilon_{s}(t)$ in (6.2.32), as compared with (6.2.23)); ii) asymptotic tracking performance is recovered, when all subsystem can be matched to an LTI system with the same transfer function as the reference model system (see Theorem 6.2.8), while this cannot be archived by the conventional MRAC design; and iii) Assumption (A6.2-4a) is not necessary.

In general, we can expect an improved tracking performance by the proposed adaptive control scheme over this conventional MRAC design, as shown by the illustrative examples given next.

\subsection{Simulation Study}

In this section a simulation study is first performed for the output tracking problem of a SISO piecewise linear system in controllable canonical form (CCF), then the proposed multivariable MRAC design in Section 6.2 is applied to the linearized lateraldirectional model of the NASA GTM to demonstrate its effectiveness in performance improvement, as compared with the conventional MRAC design. 


\subsubsection{SISO Piecewise Linear Systems in CCF}

Consider a piecewise linear system (6.1.1) of third order with three subsystems; that is, $n=3$ and $l=3$ in (6.1.1)-(6.1.3). In particular,

$$
\begin{aligned}
& \boldsymbol{A}_{1}=\left[\begin{array}{ccc}
0 & 1 & 0 \\
0 & 0 & 1 \\
2 & -3 & -5
\end{array}\right], \quad \boldsymbol{A}_{2}=\left[\begin{array}{ccc}
0 & 1 & 0 \\
0 & 0 & 1 \\
-2 & 0 & 1
\end{array}\right], \quad \boldsymbol{A}_{3}=\left[\begin{array}{ccc}
0 & 1 & 0 \\
0 & 0 & 1 \\
0 & 1 & -2
\end{array}\right] \\
& \boldsymbol{b}_{1}=\left[\begin{array}{l}
0 \\
0 \\
1
\end{array}\right], \quad \boldsymbol{b}_{2}=\left[\begin{array}{l}
0 \\
0 \\
2
\end{array}\right], \quad \boldsymbol{b}_{3}=\left[\begin{array}{c}
0 \\
0 \\
0.5
\end{array}\right], \quad \boldsymbol{c}^{\top}=\left[\begin{array}{lll}
1 & 1 & 0
\end{array}\right]
\end{aligned}
$$

It can be verified that all three subsystems are open loop unstable and minimum phase with a single zero at $s=-1$. Their respective high frequency gains are all positive. With $m=1$, the relative degree of the system is $n^{*}=n-m=2$, thus we choose the reference model system transfer function as

$$
W_{m}(s)=\frac{1}{s^{2}+2 s+1}
$$

Matching Condition and Nominal Parameters. The plant-model matching condition (6.1.7) can be satisfied with the nominal controller parameters

$$
\begin{aligned}
& \boldsymbol{k}_{x 1}^{*}=[-3,0,2]^{\top}, \quad \boldsymbol{k}_{x 2}^{*}=[0.5,-1.5,-2]^{\top}, \quad \boldsymbol{k}_{x 3}^{*}=[-2,-8,-2]^{\top} \\
& k_{r 1}^{*}=1, \quad k_{r 2}^{*}=0.5, \quad k_{r 3}^{*}=2
\end{aligned}
$$

In fact, for $i=1,2,3$,

$$
\boldsymbol{A}_{m i}=\boldsymbol{A}_{m}^{*}=\left[\begin{array}{ccc}
0 & 1 & 0 \\
0 & 0 & 1 \\
-1 & -3 & -3
\end{array}\right], \quad \boldsymbol{b}_{m i}=\boldsymbol{b}_{m}^{*}=\left[\begin{array}{l}
0 \\
0 \\
1
\end{array}\right]
$$

and $\boldsymbol{c}^{\top}\left(s \boldsymbol{I}-\boldsymbol{A}_{m}^{*}\right)^{-1} \boldsymbol{b}_{m}^{*}=W_{m}(s)$, so that the condition of Theorem 6.1 .8 is satisfied.

Simulation Results. In this simulation, a change of subsystem occurs in sequence among the three subsystems every 10 seconds, i.e., $T=10$. The initial parameter estimates $\boldsymbol{\theta}_{i}(0), \rho_{i}(0)$ in the adaptive laws (6.1.22) are chosen as $80 \%$ of 
their nominal values; that is, $\boldsymbol{\theta}_{i}(0)=0.8 \boldsymbol{\theta}_{i}^{*}, \rho_{i}(0)=0.8 \rho_{i}^{*}$. The adaptation gains are $\boldsymbol{\Gamma}_{i}=3 \boldsymbol{I}, \gamma_{i}=3$ for $i=1,2,3$. The initial state vector of the system is $\boldsymbol{x}(0)=[4,-5,3]^{\top}$, and that for the reference model is zero. To generate the normalizing signal $m(t)$ from $m_{s}(t)$ in $(6.1 .23), \delta_{0}$ is chosen to be -0.5 .

Simulations are performed for the reference input signal $r(t)$ being constant and sinusoidal, i.e., $r(t)=2$ and $r(t)=2 \sin (0.5 t)$, respectively, and the time history of the tracking error $e(t)$ is plotted in Figures 6.1-6.2 (top). As a comparison, the tracking performance with the conventional SISO state feedback output tracking MRAC scheme under the same conditions is also shown in Figures 6.1-6.2 (bottom).
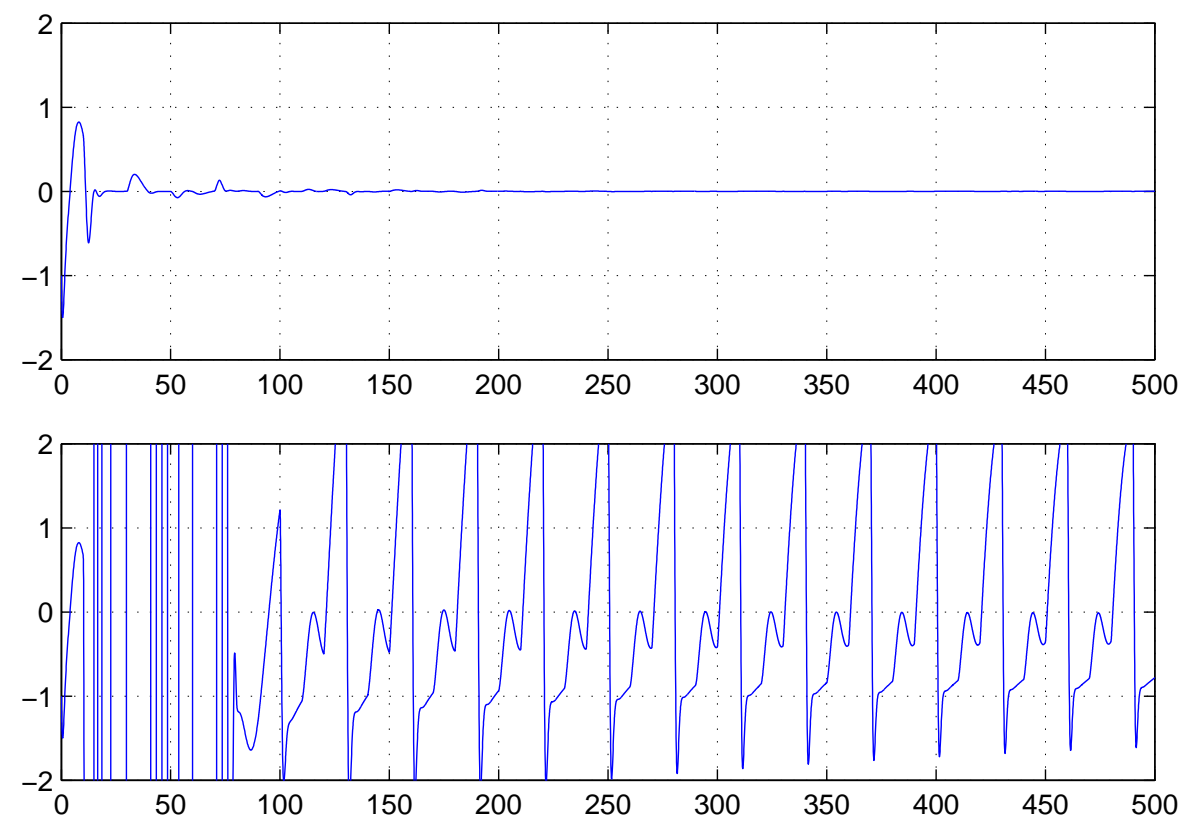

Figure 6.1: Tracking error $e(t)$ with $r(t)=2$ : the proposed scheme (top) vs. the conventional MRAC scheme (bottom).

As can be seen from the simulation results, with the proposed adaptive control scheme, both closed-loop signal boundedness and asymptotic tracking are achieved, which is a significant performance improvement compared with the conventional adap- 

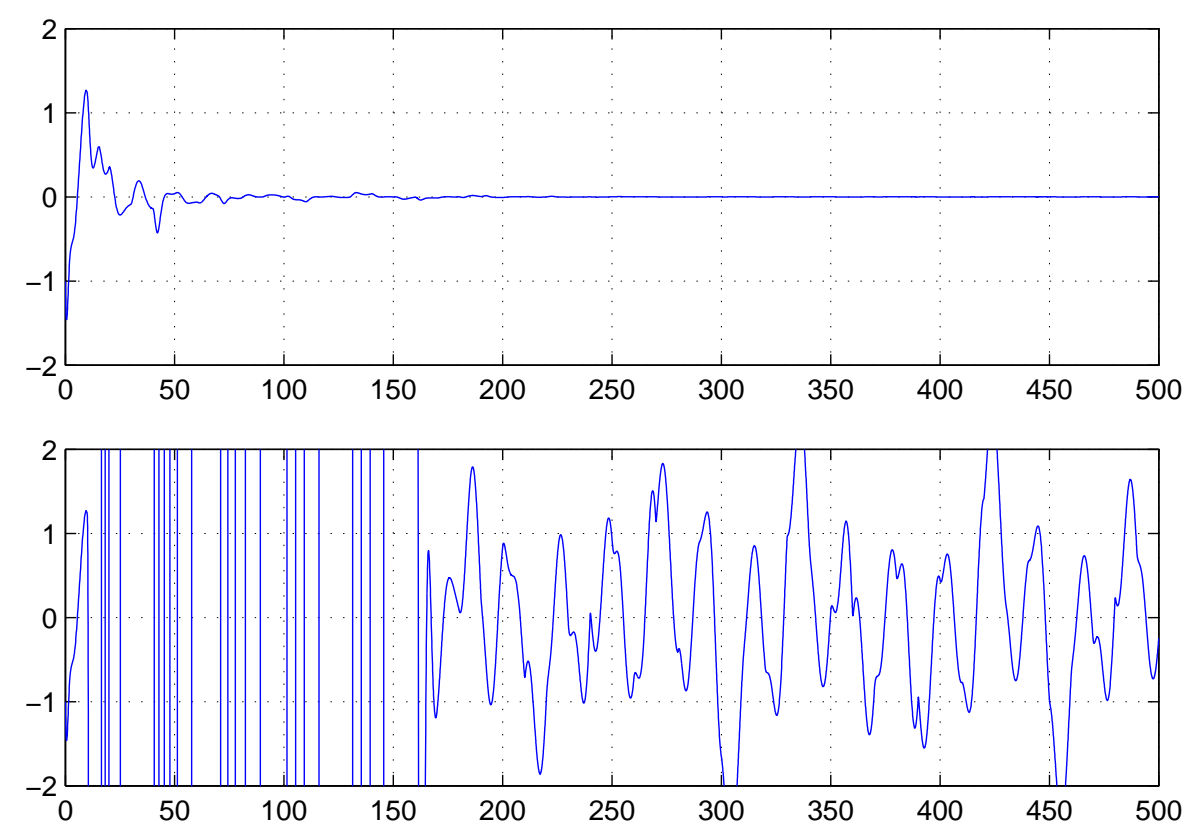

Figure 6.2: Tracking error $e(t)$ with $r(t)=2 \sin (0.5 t)$ : the proposed scheme (top) vs. the conventional MRAC scheme (bottom).

tive control scheme that does not take into account the parameter discontinuities.

\subsubsection{Linearized Lateral-Directional NASA GTM Model}

Simulations are performed to demonstrate the system stability and tracking performance with the proposed multivariable adaptive control scheme in Section 6.2 applied to a piecewise linear system model of the NASA GTM [82] at multiple operating points. It is to be noted that in the simulations, switches between the chosen linearized GTM models are time-dependent for illustration purposes, while transitions of operating points of the nonlinear GTM system are state-dependent.

Linearized Model and Reference Model System. For simplicity of presentation, we choose $l=2$, and trim the GTM at steady-state, straight, wings-level flight conditions at 80 knots and 90 knots at $800 \mathrm{ft}$., respectively, to obtain a piecewise 
linear lateral system model in the form of (6.2.1), where $\boldsymbol{x}=[v, p, r, \phi, \psi]^{\top}$ with the elements being the perturbed aircraft velocity component along the y-body-axis (fps), angular velocity along the x- and z-body-axis (crad/s), roll angle (crad), and yaw angle (crad), respectively. The outputs are chosen as $\boldsymbol{y}=[v, \psi]^{\top}$, while the control inputs are chosen as the perturbed aileron deflection $\delta_{a}$ and rudder deflection $\delta_{r}$, i.e., $\boldsymbol{u}=\left[\delta_{a}, \delta_{r}\right]^{\top}$, and the nominal parameter matrices are

$$
\begin{aligned}
& \boldsymbol{A}_{1}=\left[\begin{array}{rrrrr}
-0.6137 & 0.0959 & -1.3454 & 0.3210 & 0 \\
-66.3000 & -6.7565 & 1.8813 & 0 & 0 \\
24.1200 & -0.3162 & -1.4992 & 0 & 0 \\
0 & 1.0000 & 0.0691 & 0.0002 & 0 \\
0 & 0 & 1.0000 & 0 & 0
\end{array}\right] \\
& \boldsymbol{A}_{2}=\left[\begin{array}{rrrrr}
-0.6870 & 0.0801 & -1.5153 & 0.3213 & 0 \\
-72.9200 & -7.5625 & 1.8623 & 0 & 0 \\
27.3600 & -0.3078 & -1.6865 & 0 & 0 \\
0 & 1.0000 & 0.0513 & 0.0001 & 0 \\
0 & 0 & 1.0000 & 0 & 0
\end{array}\right] \\
& \boldsymbol{B}_{1}=\left[\begin{array}{rr}
-0.0274 & 0.4892 \\
-90.0900 & 29.6300 \\
-2.5200 & -46.8300 \\
0 & 0 \\
0 & 0
\end{array}\right], \boldsymbol{B}_{2}=\left[\begin{array}{rr}
-0.0396 & 0.6160 \\
-116.5300 & 38.2700 \\
-3.1300 & -59.5300 \\
0 & 0 \\
0 & 0
\end{array}\right], \boldsymbol{C}=\left[\begin{array}{ll}
1 & 0 \\
0 & 0 \\
0 & 0 \\
0 & 0 \\
0 & 1
\end{array}\right]
\end{aligned}
$$

It is clear that $M=2, n=5$, and with the choice of the modified left interactor matrix as

$$
\boldsymbol{\xi}_{m}(s)=\left[\begin{array}{rr}
s+1 & 0 \\
0 & (s+1)^{2}
\end{array}\right]
$$

we obtain the high frequency gain matrices

$$
\boldsymbol{K}_{p 1}=\left[\begin{array}{rr}
-0.0274 & 0.4892 \\
-2.5200 & -46.8300
\end{array}\right], \quad \boldsymbol{K}_{p 2}=\left[\begin{array}{rr}
-0.0396 & 0.6160 \\
-3.1300 & -59.5300
\end{array}\right]
$$

It then follows that $\operatorname{sign}\left[\Delta_{11}\right]=-1, \operatorname{sign}\left[\Delta_{12}\right]=1, \operatorname{sign}\left[\Delta_{21}\right]=-1, \operatorname{sign}\left[\Delta_{22}\right]=1$, and with the choice of $\boldsymbol{D}_{s 1}=-100 \boldsymbol{I}, \boldsymbol{D}_{s 2}=\operatorname{diag}\{-100,-197.8998\}$, we have

$$
\begin{aligned}
& \boldsymbol{L}_{s 1}=\left[\begin{array}{rl}
1 & 0 \\
109.8248 & 1
\end{array}\right], \boldsymbol{S}_{1}=\left[\begin{array}{rr}
0.0003 & -0.0049 \\
-0.0049 & 1.0056
\end{array}\right] \\
& \boldsymbol{L}_{s 2}=\left[\begin{array}{rr}
1 & 0 \\
109.8248 & 1
\end{array}\right], \boldsymbol{S}_{2}=\left[\begin{array}{rr}
0.0004 & -0.0062 \\
-0.0062 & 0.6427
\end{array}\right]
\end{aligned}
$$


With $\boldsymbol{\xi}_{m}(s)$ as above, the reference model system is specified by

$$
\boldsymbol{y}_{m}(t)=\left[\begin{array}{rr}
\frac{1}{s+1} & 0 \\
0 & \frac{1}{(s+1)^{2}}
\end{array}\right][\boldsymbol{r}](t)
$$

where $\boldsymbol{r}(t)$ is a bounded, piecewise continuous reference input vector signal.

Matching Condition and Nominal Parameters. It can be verified that the plant model matching condition (6.2.7) for $i=1,2$, is satisfied with

$$
\begin{aligned}
& \boldsymbol{K}_{x 1}^{*}=\left[\begin{array}{rr}
11.8803 & -0.1242 \\
1.7235 & -0.0995 \\
-24.9447 & 1.3530 \\
5.9744 & -0.3215 \\
0.1944 & 0.0109
\end{array}\right], \quad \boldsymbol{K}_{x 2}^{*}=\left[\begin{array}{rr}
8.2807 & 0.0242 \\
1.0678 & -0.0613 \\
-21.0040 & 1.1096 \\
4.4635 & -0.2347 \\
0.1437 & 0.0092
\end{array}\right] \\
& \boldsymbol{K}_{r 1}^{*}=\left[\begin{array}{rr}
-18.6134 & -0.1944 \\
1.0016 & -0.0109
\end{array}\right], \quad \boldsymbol{K}_{r 2}^{*}=\left[\begin{array}{rr}
-13.8911 & -0.1437 \\
0.7304 & -0.0092
\end{array}\right]
\end{aligned}
$$

and we have $\theta_{2}^{*}=-109.8248, \boldsymbol{\Psi}_{i}^{*}=\boldsymbol{D}_{s i} \boldsymbol{S}_{i}, i=1,2$.

Simulation Parameters. In this simulation, we choose a switching time interval $T=200 \mathrm{~s}$. The initial plant state is $\boldsymbol{x}_{0}=[2,0,0,0,-10]^{\top}$ with zero reference model initial condition, and the initial parameter estimates are chosen to be $90 \%$ of their nominal values; that is, for $i=1,2$ :

$$
\theta_{2}(0)=90 \% \theta_{2}^{*}, \quad \boldsymbol{\Theta}_{i}(0)=90 \% \boldsymbol{\Theta}_{i}^{*}, \quad \boldsymbol{\Psi}_{i}(0)=90 \% \boldsymbol{\Psi}_{i}^{*}
$$

The adaptation gains are chosen as $\Gamma_{\theta_{2}}=10^{3}, \boldsymbol{\Gamma}_{1}=\boldsymbol{\Gamma}_{2}=10^{5} \boldsymbol{I}$. The parameters of the signal filter $h(s)$ are chosen such that $f(s)=(s+6)^{2}$.

Simulation I: $\boldsymbol{r}(t)=[1,10]^{\top}$. Figure 6.3 shows the output tracking error $\boldsymbol{e}(t)$ with the constant reference input $\boldsymbol{r}(t)=[1,10]^{\top}$, corresponding to a desired steadystate lateral velocity of 1 fps and a yaw angle of $5.73^{\circ}$ (dashed), despite the switches of operating points every 200s. The aileron and rudder deflections are plotted in Figure 6.4. They are within their respective limits $\left( \pm 20^{\circ}\right.$ and $\left.\pm 30^{\circ}\right)$. As a comparison, 
simulations are run for the conventional MRAC scheme applied to the same system under the same conditions. The output tracking performance and control inputs are plotted in Figures 6.5-6.6.
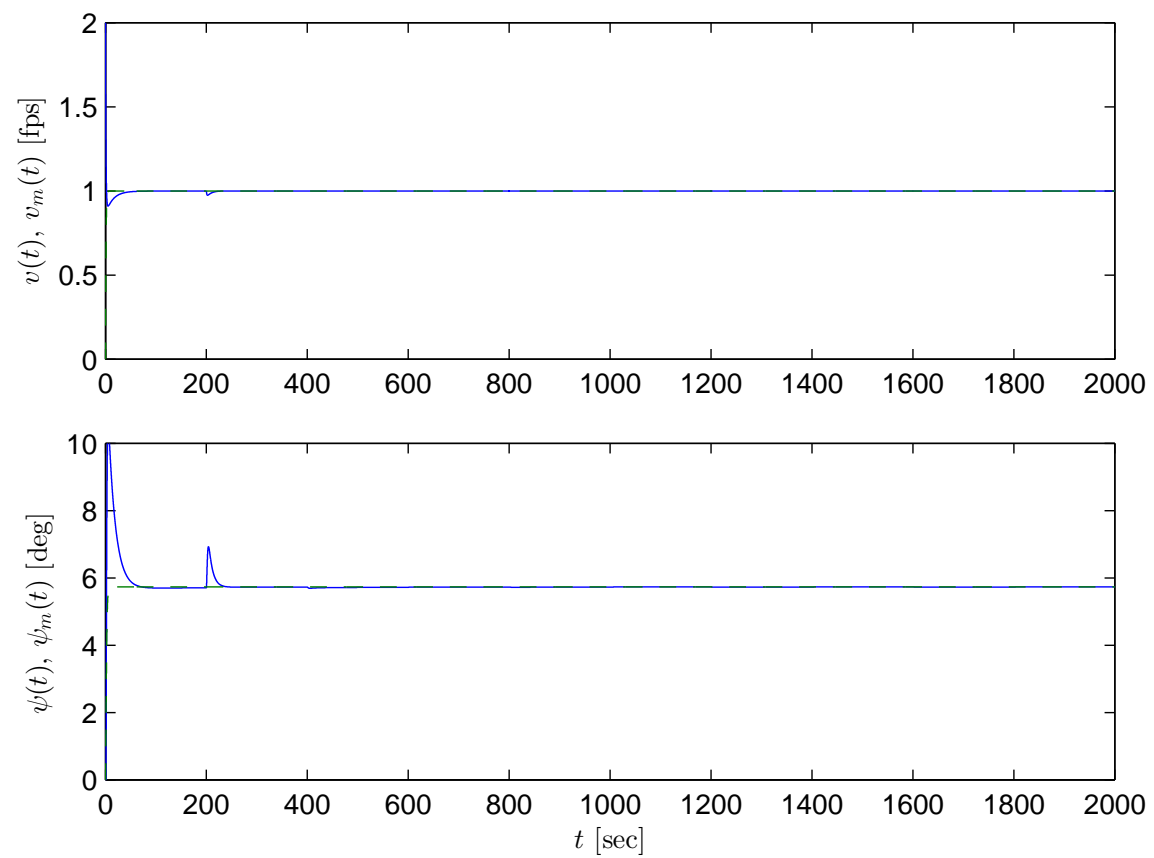

Figure 6.3: Simulation I: tracking performance of the proposed scheme.

Simulation II: $\boldsymbol{r}(t)=\sin (0.014 t)[1,10]^{\top}$. Figure 6.7 shows the output tracking error $\boldsymbol{e}(t)$ with the sinusoidal reference input $\boldsymbol{r}(t)=\sin (0.014 t)[1,10]^{\top}$, corresponding to desired fluctuations of the lateral velocity in between $\pm 1 \mathrm{fps}$ and of the yaw angle in between $\pm 5.73^{\circ}$ (dashed), despite the switches of operating points every 200s. The aileron and rudder deflections are plotted in Figure 6.8. They are within their respective limits. The simulation results for the conventional MRAC scheme under the same conditions are plotted in Figures 6.9-6.10.

It can be seen from the simulation results that although closed-loop stability is achieved by both adaptive control schemes and the control inputs are within the 

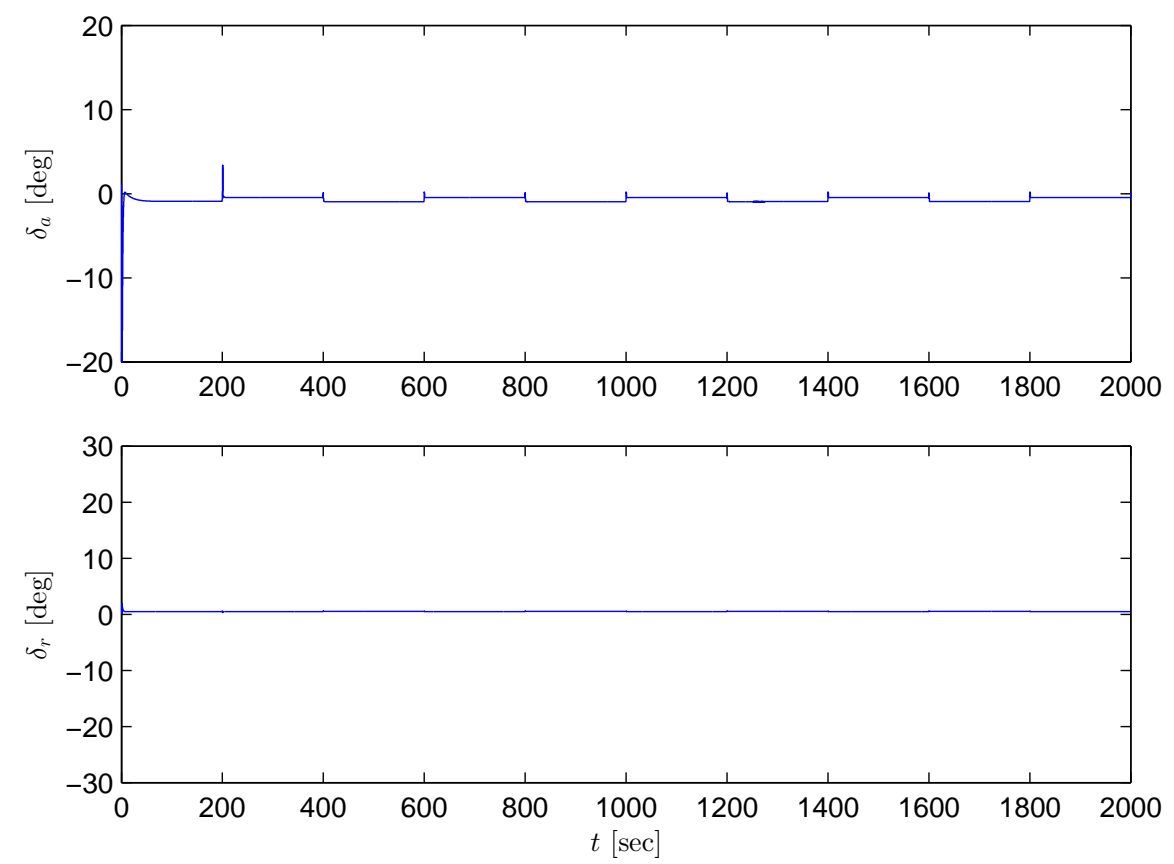

Figure 6.4: Simulation I: control input $\boldsymbol{u}(t)$ of the proposed scheme.
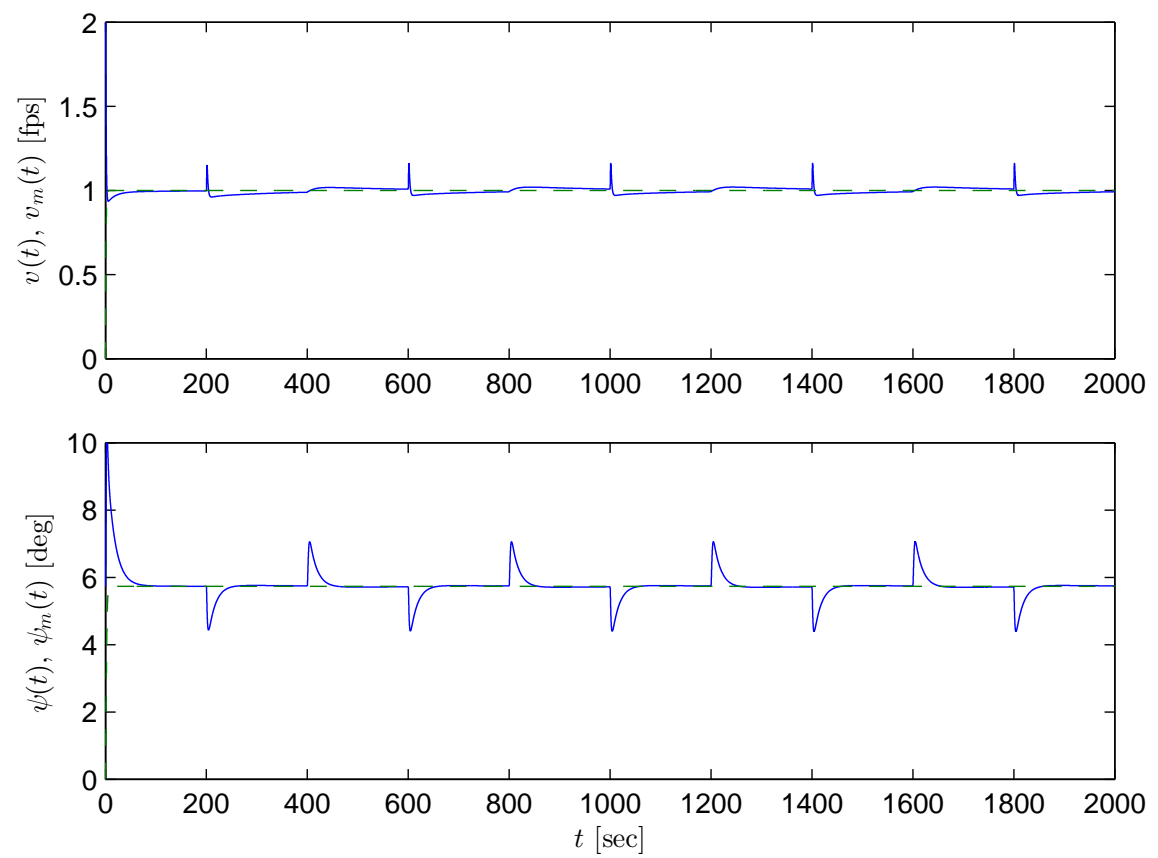

Figure 6.5: Simulation I: tracking performance of the conventional MRAC scheme. 

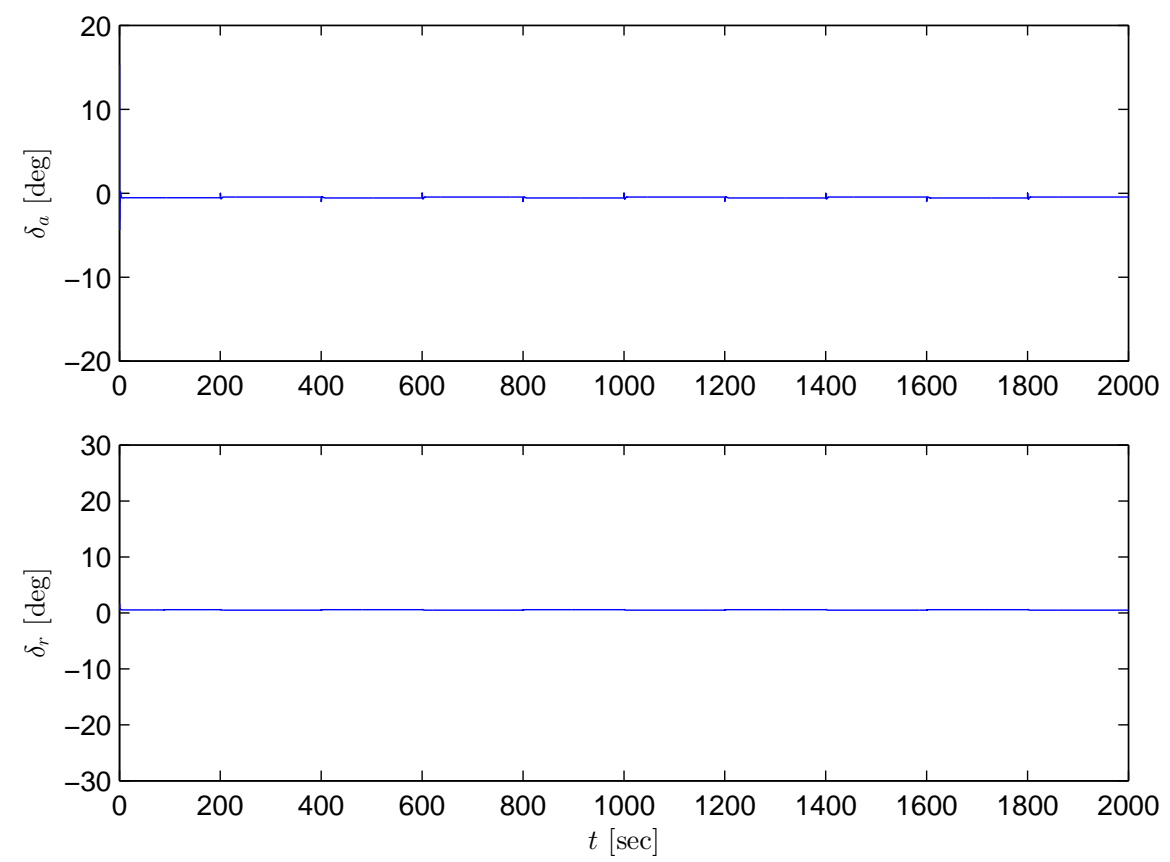

Figure 6.6: Simulation I: control input $\boldsymbol{u}(t)$ of the conventional MRAC scheme.
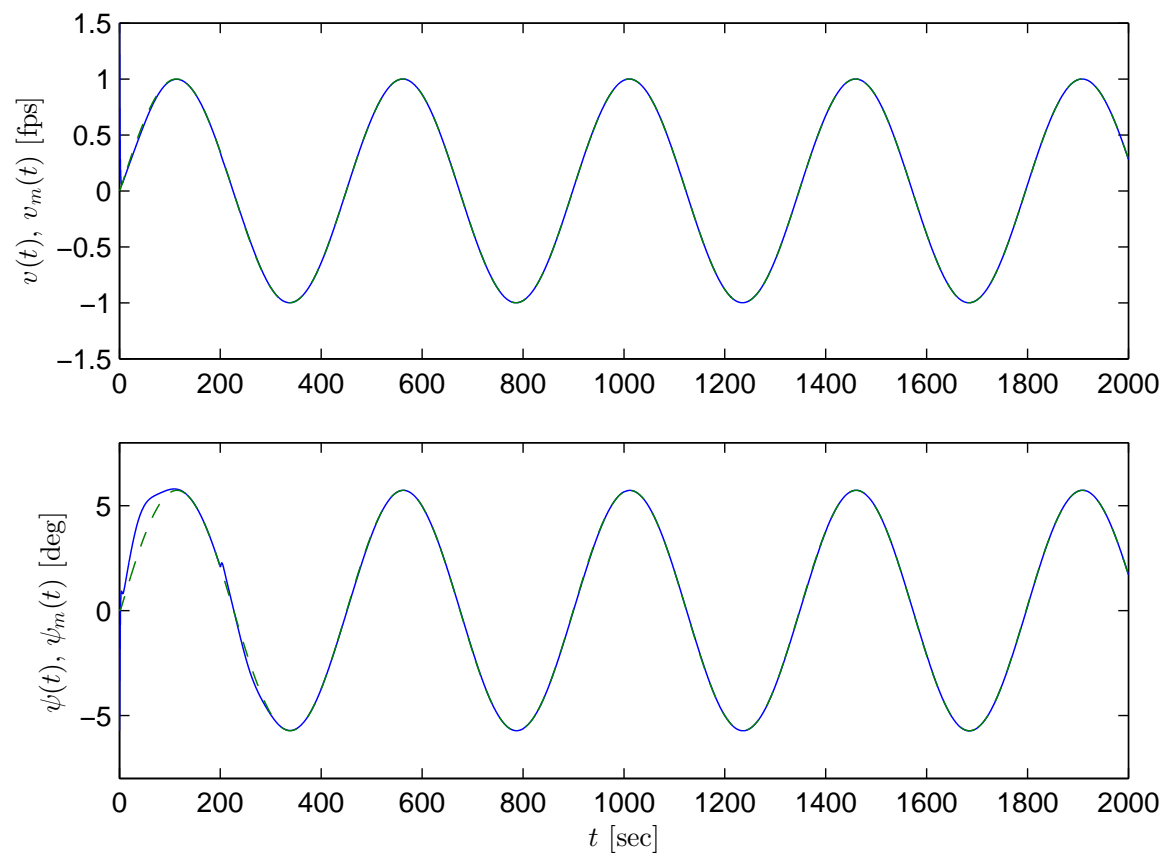

Figure 6.7: Simulation II: tracking performance of the proposed scheme. 

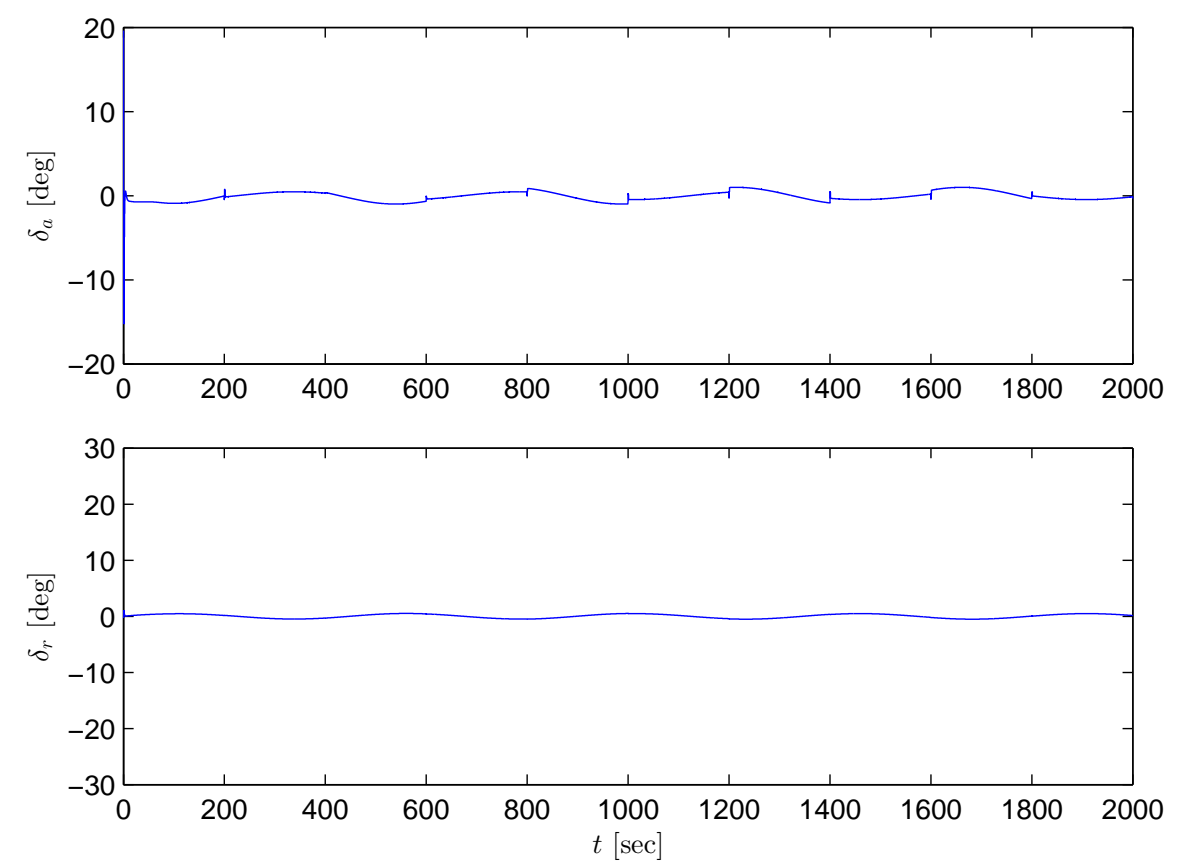

Figure 6.8: Simulation II: control input $\boldsymbol{u}(t)$ of the proposed scheme.
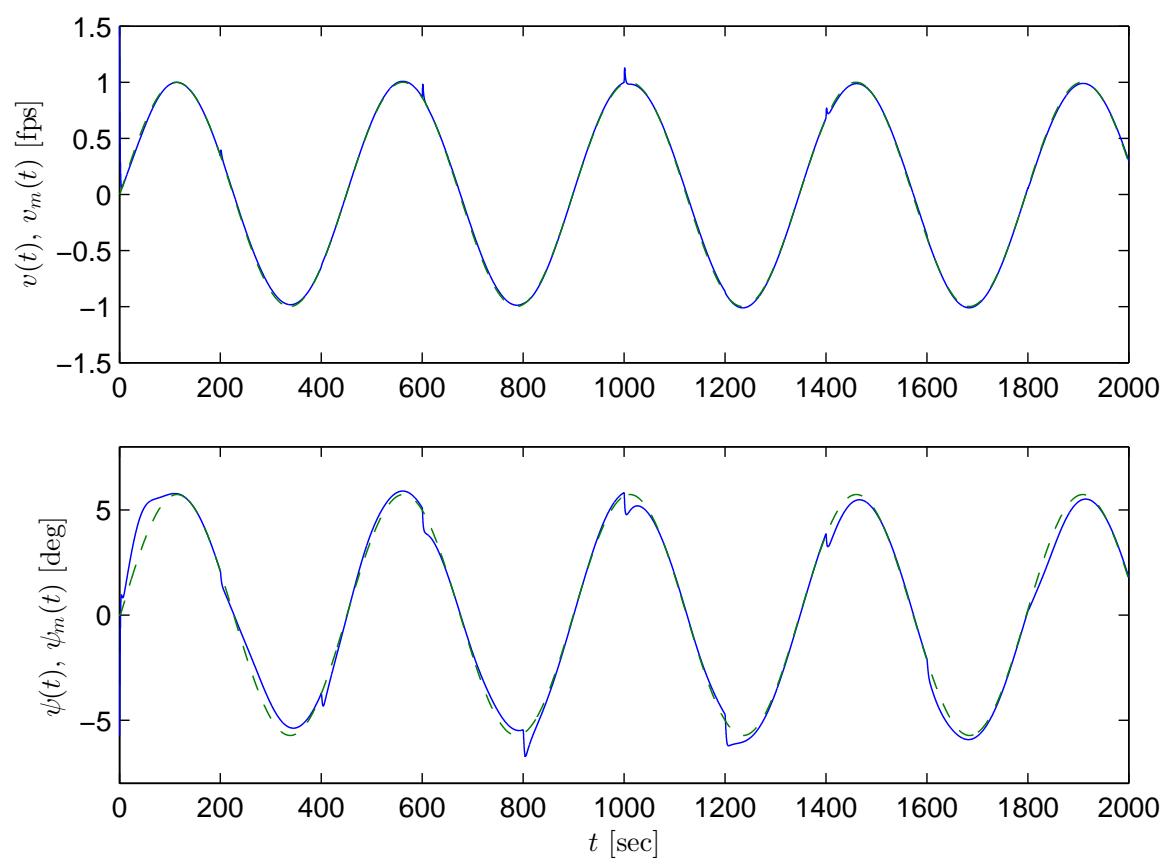

Figure 6.9: Simulation II: tracking performance of the conventional MRAC scheme. 

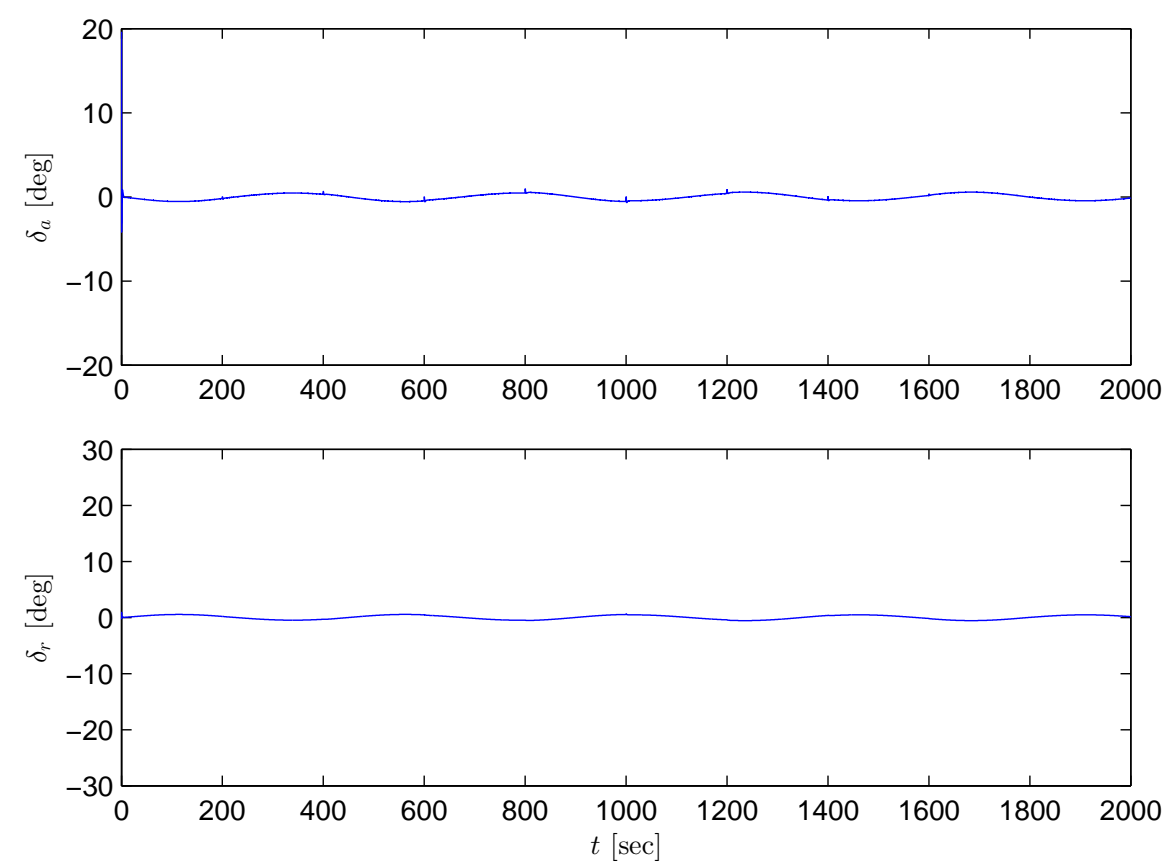

Figure 6.10: Simulation II: control input $\boldsymbol{u}(t)$ of the conventional MRAC scheme.

required limits, the proposed design outperforms the conventional MRAC design in tracking performance.

In this chapter, direct model reference adaptive control (MRAC) schemes are developed for both SISO and MIMO piecewise linear systems. Closed-loop signal boundedness and small output tracking error in the mean square sense are achieved via state feedback for sufficiently slow subsystem switches. Simulation results demonstrate the effectiveness of the proposed adaptive control scheme in performance improvement, as compared with conventional MRAC schemes. 


\section{Chapter 7}

\section{Adaptive Control of Piecewise Linear Systems with Output Feedback for Output Tracking}

In this chapter, the adaptive output feedback control problem is studied for singleinput, single-output (SISO) piecewise linear systems with switched parameters. By employing the knowledge of the time instant indicator functions of system parameter switching, a new controller structure parametrization is proposed for the development of a stable adaptive control scheme which is capable of reducing the modeling error in the estimation error signal used for parameter adaptive laws. This key feature is achieved by the new controller scheme's ability to avoid a major parameter swapping term in the error model, with the help of indicator functions whose knowledge is available in many applications. It is shown that under the usual slow system parameter switching condition, the closed-loop stability (signal boundedness) and small output tracking error in the mean square sense are achieved. Simulation results on linearized NASA GTM models are presented, demonstrating the effectiveness and performance improvement of the proposed adaptive control scheme. 


\subsection{Problem Statement}

Consider a single-input, single-output (SISO) piecewise linear system

$$
\begin{aligned}
\dot{\boldsymbol{x}}(t) & =\boldsymbol{A}(t) \boldsymbol{x}(t)+\boldsymbol{b}(t) u(t), \quad \boldsymbol{x}\left(t_{0}\right)=\boldsymbol{x}_{0} \\
y(t) & =\boldsymbol{c}^{\top}(t) \boldsymbol{x}(t)
\end{aligned}
$$

where $\boldsymbol{x}(t) \in \mathbb{R}^{n}$ is the state vector, and $u(t) \in \mathbb{R}$ and $y(t) \in \mathbb{R}$ are the control input and the controlled output, respectively, which are the only signals available for measurement. The system parameter matrices (vectors) $\boldsymbol{A}(t) \in \mathbb{R}^{n \times n}, \boldsymbol{b}(t) \in \mathbb{R}^{n}$ and $\boldsymbol{c}(t) \in \mathbb{R}^{n}$ are unknown, and vary in a piecewise constant pattern; that is, during different time periods, $\left(\boldsymbol{c}^{\boldsymbol{\top}}(t), \boldsymbol{A}(t), \boldsymbol{b}(t)\right)$ take on different values as specified by the parameter matrix sets $\left(\boldsymbol{c}_{i}^{\top}, \boldsymbol{A}_{i}, \boldsymbol{b}_{i}\right), i \in \mathcal{I} \triangleq\{1,2, \ldots, l\}$, where $\boldsymbol{A}_{i}, \boldsymbol{b}_{i}$, and $\boldsymbol{c}_{i}$ are unknown but constant parameter matrices, and $l$ is the total number of subsystems. To characterize such time-varying behaviors of the system, we introduce the indicator functions as follows.

Indicator Functions. The indicator functions $\chi_{i}(t)$ are assumed to be known and defined as

$$
\chi_{i}(t)= \begin{cases}1, & \text { if }\left(\boldsymbol{c}^{\boldsymbol{\top}}(t), \boldsymbol{A}(t), \boldsymbol{b}(t)\right)=\left(\boldsymbol{c}_{i}^{\top}, \boldsymbol{A}_{i}, \boldsymbol{b}_{i}\right) \\ 0, & \text { otherwise }\end{cases}
$$

It follows that $\sum_{i=1}^{l} \chi_{i}(t)=1, \chi_{j}(t) \chi_{k}(t)=0, j \neq k$. With the indicator functions $\chi_{i}(t)$, the time-varying plant parameter matrices $\boldsymbol{A}(t), \boldsymbol{b}(t), \boldsymbol{c}(t)$ can be expressed as

$$
\boldsymbol{A}(t)=\sum_{i=1}^{l} \boldsymbol{A}_{i} \chi_{i}(t), \boldsymbol{b}(t)=\sum_{i=1}^{l} \boldsymbol{b}_{i} \chi_{i}(t), \boldsymbol{c}(t)=\sum_{i=1}^{l} \boldsymbol{c}_{i} \chi_{i}(t)
$$

Control Objective. The control objective is to develop an output feedback control law $u(t)$ for the piecewise linear system (7.1.1) with parameter variations characterized as in (7.1.3) such that all the signals in the closed-loop system are 
bounded, and the system output $y(t)$ tracks as close as possible a reference trajectory $y_{m}(t)$, which is generated from a reference model system

$$
y_{m}(t)=W_{m}(s)[r](t), \quad W_{m}(s)=\frac{1}{P_{m}(s)}
$$

where $P_{m}(s)$, independent of the system parameters, is a desired closed-loop characteristic polynomial of degree $n^{*}$, and $r(t)$ is an external reference input signal which is bounded and piecewise continuous.

MRAC Assumptions. Let the strictly increasing sequence $\left\{t_{k}\right\}_{k=1}^{\infty}$ denote the time instants at which subsystem switches occur. Within each switching time interval $\left[t_{k-1}, t_{k}\right)$, the system input/output (I/O) mapping, $u(t) \mapsto y(t)$, can be described by the following transfer function (supposing the $i$ th subsystem is active over this time interval)

$$
G_{i}(s)=\frac{k_{p i} Z_{i}(s)}{P_{i}(s)}
$$

where $P_{i}(s)=s^{n}+p_{n-1 i} s^{n-1}+\cdots+p_{1 i} s+p_{0 i}$ and $Z_{i}(s)=s^{m}+z_{m-1 i} s^{m-1}+\cdots+z_{1 i} s+z_{0 i}$ are monic polynomials with unknown constant coefficients, and $k_{p i} \neq 0$.

To design an adaptive output feedback control law for output tracking, the following assumptions are made for $i \in \mathcal{I}$ :

(A7.1-1) $\left(\boldsymbol{A}_{i}, \boldsymbol{b}_{i}\right)$ is controllable and $\left(\boldsymbol{c}_{i}^{\top}, \boldsymbol{A}_{i}\right)$ is observable;

(A7.1-2) The zeros of $G_{i}(s)$ are stable with their real parts less than $-\alpha$ for some known $\alpha>0$;

(A7.1-3) The degree of $P_{i}(s), \operatorname{deg}\left[P_{i}(s)\right]=n$, is known;

(A7.1-4) The degree of $P_{m}(s), \operatorname{deg}\left[P_{m}(s)\right]=n^{*}=n-m$, is known;

(A7.1-5) The sign of $k_{p i}, \operatorname{sign}\left[k_{p i}\right]$, is known; and 
(A7.1-6) There exists positive constants $C$ and $\mu$ such that the number $N(T)$ of subsystem switches within any time interval $(t, t+T)$ satisfies $N(T) \leq C+\mu T$, $\forall t \geq t_{0}, T \geq 0$.

Remark 7.1.1. The I/O description (7.1.5) is not a complete characterization of the plant behavior, because it does not include the boundary conditions at the switching time instants; that is, the output $y(t)$ also depends on the internal system states at $\left\{t_{k}\right\}_{k=1}^{\infty}$, due to the presence of system parameter discontinuities. Nevertheless, such a piecewise I/O description is useful when studying the piecewise system matching condition for the adaptive control design and stability analysis.

Remark 7.1.2. The requirement of each subsystem being controllable and observable in Assumption (A7.1-1) is to ensure that the state-space description of the system is minimal and any transients appearing in the closed-loop system dynamics due to subsystem switches can be fully characterized by the zeros of $Z_{i}(s), P_{m}(s)$, and $\Lambda(s)$ (characterizing filter dynamics used for control implementation).

Remark 7.1.3. As will be shown later, subsystem switches lead to the appearance of the internal system state at switching time instants in the output signal $y(t)$. The knowledge of $\alpha$ in Assumption (A7.1-2) is required such that a suitable dynamic normalizing signal can be designed, and the boundedness property of the normalized state vector can be used in concluding the closed-loop stability properties.

Remark 7.1.4. Inherent in Assumptions (A7.1-3) and (A7.1-4) is that the system order $n$ in each subsystem is a known constant, so is the relative degree $n-m$, which is the same as that $\left(n^{*}\right)$ of the reference model system.

Remark 7.1.5. The constant $\mu$ in Assumption (A7.1-6) is to characterize the average frequency of subsystem switches over a time interval of length $T$. It is used mainly for 
two purposes: for the nominal part of the closed-loop system, there exists an upper bound of $\mu$ which ensures that piecewise exponential stability (over each switching time interval) implies exponential stability. Further, the effects of subsystem switches can be characterized by $\mu$ in a mean square sense, and sufficiently small $\mu$ ensures the smallness of the loop gain of the closed-loop system, thus leading to closed-loop stability.

\subsection{Nominal Control Design}

The model reference control problem is considered in this section for the case when the plant parameter matrices are known exactly.

\subsubsection{Controller Structure}

If the plant parameter matrices were known, the following nominal control law was proposed in [83]:

$$
u(t)=\frac{\boldsymbol{a}^{\boldsymbol{\top}}(s)}{\Lambda(s)}\left[\boldsymbol{\theta}_{1}^{*} u\right](t)+\frac{\boldsymbol{a}^{\boldsymbol{\top}}(s)}{\Lambda(s)}\left[\boldsymbol{\theta}_{2}^{*} y\right](t)+\theta_{20}^{*}(t) y(t)+\theta_{3}^{*}(t) r(t)
$$

where the controller parameter vectors $\boldsymbol{\theta}_{1}^{*}(t), \boldsymbol{\theta}_{2}^{*}(t) \in \mathbb{R}^{n-1}, \theta_{20}^{*}(t), \theta_{3}^{*}(t) \in \mathbb{R}$ are piecewise continuous, and are computed from some design equations, $\Lambda(s)$ is a chosen monic stable polynomial of degree $n-1$, e.g., $\Lambda(s)=s^{n-1}+\lambda_{n-2} s^{n-2}+\cdots+\lambda_{1} s+\lambda_{0}$, and $\boldsymbol{a}(s)=\left[1, s, \ldots, s^{n-2}\right]^{\top}$. The nominal control law (7.2.1) may be implemented as

$$
u(t)=\boldsymbol{b}_{\lambda}^{\top} \boldsymbol{\omega}_{1}(t)+\boldsymbol{b}_{\lambda}^{\top} \boldsymbol{\omega}_{2}(t)+\theta_{20}^{*}(t) y(t)+\theta_{3}^{*}(t) r(t)
$$

with $\boldsymbol{\omega}_{1}(t)$ and $\boldsymbol{\omega}_{2}(t)$ generated from

$$
\begin{array}{ll}
\dot{\boldsymbol{\omega}}_{1}(t)=\boldsymbol{A}_{\lambda}^{\top} \boldsymbol{\omega}_{1}(t)+\boldsymbol{\theta}_{1}^{*}(t) u(t), & \boldsymbol{\omega}_{1}\left(t_{0}\right)=\mathbf{0} \\
\dot{\boldsymbol{\omega}}_{2}(t)=\boldsymbol{A}_{\lambda}^{\top} \boldsymbol{\omega}_{2}(t)+\boldsymbol{\theta}_{2}^{*}(t) y(t), & \boldsymbol{\omega}_{2}\left(t_{0}\right)=\mathbf{0}
\end{array}
$$


and $\left(\boldsymbol{A}_{\lambda}, \boldsymbol{b}_{\lambda}\right)$ is in controllable canonical form, i.e.,

$$
\boldsymbol{A}_{\lambda}=\left[\begin{array}{ccccc}
0 & 1 & 0 & \cdots & 0 \\
0 & 0 & 1 & \cdots & 0 \\
\vdots & \vdots & \vdots & \ddots & \vdots \\
-\lambda_{0} & -\lambda_{1} & -\lambda_{2} & \cdots & -\lambda_{n-2}
\end{array}\right], \quad \boldsymbol{b}_{\lambda}=\left[\begin{array}{c}
0 \\
0 \\
\vdots \\
1
\end{array}\right]
$$

such that $\boldsymbol{b}_{\lambda}^{\top}\left(s \boldsymbol{I}_{n}-\boldsymbol{A}_{\lambda}^{\top}\right)^{-1}=\boldsymbol{a}^{\top}(s) / \Lambda(s)$.

This control law was originally proposed in [83] in the context of linear timevarying systems with smooth parameter variations. It is motivated by and reduces in the time-invariant case to the standard output feedback control law $[9,15]$ :

$$
u(t)=\boldsymbol{\theta}_{1}^{* \mathrm{~T}} \frac{\boldsymbol{a}(s)}{\Lambda(s)}[u](t)+\boldsymbol{\theta}_{2}^{* \mathrm{~T}} \frac{\boldsymbol{a}(s)}{\Lambda(s)}[y](t)+\theta_{20}^{*} y(t)+\theta_{3}^{*} r(t)
$$

with constant parameter vectors $\boldsymbol{\theta}_{i}^{*}, i=1,2,20,3$. Its structural advantage, however, is the placement of parameter vectors, $\boldsymbol{\theta}_{i}^{*}, i=1,2$, with $u(t)$ and $y(t)$, before going through the filtering process $\boldsymbol{a}^{\top}(s) / \Lambda(s)$, which is key to a perfect closed-loop plantmodel matching in the time-varying case. The model reference controller (7.2.1) was later applied to plants with non-smooth and "jump" parameter variation cases [57], for which the nominal controller parameters are computed in a piecewise sense, that is, from a matching condition over each time interval without subsystem switch. In particular, for the piecewise linear system (7.1.1) with the I/O description of each subsystem in (7.1.5), the controller parameter vectors of (7.2.1) are expressed as

$$
\begin{aligned}
& \boldsymbol{\theta}_{1}^{*}(t)=\sum_{i=1}^{l} \boldsymbol{\theta}_{1 i}^{*} \chi_{i}(t), \quad \boldsymbol{\theta}_{2}^{*}(t)=\sum_{i=1}^{l} \boldsymbol{\theta}_{2 i}^{*} \chi_{i}(t) \\
& \theta_{20}^{*}(t)=\sum_{i=1}^{l} \theta_{20 i}^{*} \chi_{i}(t), \quad \theta_{3}^{*}(t)=\sum_{i=1}^{l} \theta_{3 i}^{*} \chi_{i}(t)
\end{aligned}
$$

where the indicator functions $\chi_{i}(t)$ are defined in (7.1.2), and the constant parameter vectors $\boldsymbol{\theta}_{1 i}^{*}, \boldsymbol{\theta}_{2 i}^{*} \in \mathbb{R}^{n-1}, \theta_{20 i}^{*}, \theta_{3 i}^{*} \in \mathbb{R}$ are computed from the plant-model matching 
equations:

$$
\boldsymbol{\theta}_{1 i}^{* \top} \boldsymbol{a}(s) P_{i}(s)+\left(\boldsymbol{\theta}_{2 i}^{* \top} \boldsymbol{a}(s)+\theta_{20 i}^{*} \Lambda(s)\right) k_{p i} Z_{i}(s)=\Lambda(s)\left(P_{i}(s)-k_{p i} \theta_{3 i}^{*} Z_{i}(s) P_{m}(s)\right)
$$

\subsubsection{Stability Properties}

It is worth noting that unlike conventional MRAC developed for LTI systems, the control law (7.2.1) designed based on the piecewise matching equations may not ensure stability without imposing additional conditions on the frequency of subsystem switches (e.g., $\mu$ in Assumption (A7.1-6) is sufficiently small). Even if the closed-loop system is stable, we cannot expect asymptotic tracking performance. This is because the piecewise matching equations (7.2.5) do not incorporate the boundary conditions at each subsystem switching time instant $t_{k}$, determined by the internal state of the closed-loop system:

$$
\dot{\boldsymbol{x}}_{c}(t)=\boldsymbol{A}_{c}(t) \boldsymbol{x}_{c}(t)+\boldsymbol{b}_{c}(t) r(t), y(t)=\boldsymbol{c}_{c}^{\top}(t) \boldsymbol{x}_{c}(t)
$$

where $\boldsymbol{A}_{c}(t), \boldsymbol{b}_{c}(t)$, and $\boldsymbol{c}_{c}(t)$ vary in a piecewise constant pattern among the $l$ closeloop subsystems, denoted by $\left(\boldsymbol{c}_{c i}^{\top}, \boldsymbol{A}_{c i}, \boldsymbol{b}_{c i}\right)$, according to the indicator functions $\chi_{i}(t)$, and $\boldsymbol{x}_{c}(t) \triangleq\left[\boldsymbol{x}(t), \boldsymbol{\omega}_{1}(t), \boldsymbol{\omega}_{2}(t)\right]^{\top} \in \mathbb{R}^{3 n-2}$.

Under restrictions on the frequency of subsystem switches, however, we can expect closed-loop system stability (signal boundedness) and the output tracking performance as summarized in the following theorem:

Theorem 7.2.1. Consider the closed-loop system consisting of (7.1.1) and (7.2.2) under Assumptions (A7.1-1)-(A7.1-6) with controller parameters computed from (7.2.5) in a piecewise sense. Then there exists $\mu_{0}>0$ such that $\forall \mu \in\left[0, \mu_{0}\right)$, all signals in the closed-loop system are bounded, and

$$
\int_{t}^{t+T}\left(y(\tau)-y_{m}(\tau)\right)^{2} d \tau \leq C_{1}+K_{1} \mu T, \quad \forall t \geq t_{0}, \quad \forall T>0
$$


for some $C_{1}, K_{1}>0$.

Proof: Let $\left(\boldsymbol{c}_{c(k-1)}^{\top}, \boldsymbol{A}_{c(k-1)}, \boldsymbol{b}_{c(k-1)}\right) \in\left\{\left(\boldsymbol{c}_{c i}^{\top}, \boldsymbol{A}_{c i}, \boldsymbol{b}_{c i}\right)\right\}_{i=1}^{l}$ denote the subsystem over $\left[t_{k-1}, t_{k}\right), k \in \mathbb{Z}^{+}$, and $\boldsymbol{\Phi}_{c}(t, \tau)$ be the state transition matrix associated with (7.2.6). Based on the matching equation (7.2.5), it follows that $\boldsymbol{A}_{c(k-1)}$ is exponentially stable over $\left[t_{k-1}, t_{k}\right)$, with the decay rate depending on $P_{m}(s), Z(s)$ and $\Lambda(s)$, and $\left(\boldsymbol{c}_{c(k-1)}^{\top}, \boldsymbol{A}_{c(k-1)}, \boldsymbol{b}_{c(k-1)}\right)$ is a non-minimal realization of $W_{m}(s)=1 / P_{m}(s)$. Therefore, we have

$$
\boldsymbol{c}_{c(k-1)}^{\boldsymbol{\top}} \int_{t_{k-1}}^{t} \boldsymbol{\Phi}_{c}(t, \tau) \boldsymbol{b}_{c(k-1)} r(\tau) d \tau=\boldsymbol{c}_{m}^{\top} \int_{t_{k-1}}^{t} \boldsymbol{\Phi}_{m}(t, \tau) \boldsymbol{b}_{m} r(\tau) d \tau
$$

where $\left(\boldsymbol{c}_{m}^{\top}, \boldsymbol{A}_{m}, \boldsymbol{b}_{m}\right)$ is a state space realization of the reference model system (7.1.4) with obvious notational meaning, and $\boldsymbol{\Phi}_{m}(t, \tau)$ is the associated state transition matrix: $\boldsymbol{\Phi}_{m}(t, \tau)=e^{\boldsymbol{A}_{m}(t-\tau)}$.

The output tracking error $e(t)=y(t)-y_{m}(t)$ is

$$
\begin{aligned}
e(t) & =\boldsymbol{c}_{c(k-1)}^{\top} \boldsymbol{\Phi}_{c}\left(t, t_{k-1}\right) \boldsymbol{x}_{c}\left(t_{k-1}\right)-\boldsymbol{c}_{m}^{\top} \boldsymbol{\Phi}_{m}\left(t, t_{k-1}\right) \boldsymbol{x}_{m}\left(t_{k-1}\right) \\
& =\eta_{0}(t)+\varepsilon_{0}(t)
\end{aligned}
$$

where $\varepsilon_{0}(t)$ is an initial condition related term, i.e.,

$$
\varepsilon_{0}(t)=\boldsymbol{c}_{c(k-1)}^{\top} \boldsymbol{\Phi}_{c}\left(t, t_{0}\right) \boldsymbol{x}_{c}\left(t_{0}\right)-\boldsymbol{c}_{m}^{\top} \boldsymbol{\Phi}_{m}\left(t, t_{0}\right) \boldsymbol{x}_{m}\left(t_{0}\right)
$$

and $\eta_{0}(t)$ is a sum of $k-1$ terms:

$$
\eta_{0}(t)=\sum_{t_{1}<t_{k-1} \leq t}^{t_{k-1}} \int_{t_{k-2}}^{t_{k-1}}\left[\boldsymbol{c}_{c(k-1)}^{\top} \boldsymbol{\Phi}_{c}(t, \tau) \boldsymbol{b}_{c(k-1)}-\boldsymbol{c}_{m}^{\top} \boldsymbol{\Phi}_{m}(t, \tau) \boldsymbol{b}_{m}\right] r(\tau) d \tau
$$

each corresponding to a subsystem switch. Clearly, whenever there is a subsystem switch, a deviation of $y(t)$ from the reference output trajectory $y_{m}(t)$ appears due to the $\eta_{0}(t)$ component in $e(t)$.

There exist $\mu_{0}>0$ such that $\forall \mu \in\left[0, \mu_{0}\right)$ (see Assumption (A7.1-6)), $\boldsymbol{\Phi}_{c}(t, \tau)$ is exponentially stable, thus all signals in the system are bounded $[11,39]$. The result of 
the small-in-the-mean-square output tracking performance follows by dividing $[t, t+T]$ into a sum of switching time intervals within $[t, t+T]$, and conducting and combining the integrations inside each of them.

The piecewise matching equation (7.2.5) does not imply perfect plant-model matching, and subsystem switches lead to the appearance of $\eta_{0}(t)$ in $e(t)$. For adaptive control design, such a term is treated as a perturbation to the parameterized part of an appropriately defined estimation error signal, and its impact on the closed-loop system is addressed by robust adaptive laws, e.g., those with parameter projection and $\sigma$-modification [11], to ensure bounded controller parameter estimates.

\subsection{Adaptive Control Design}

In this section, an output feedback controller structure with adaptive laws to update its parameters are presented for the piecewise linear system (7.1.1) to achieve the control objective.

\subsubsection{Controller Structure}

In practice the system parameters are rarely known exactly, therefore the nominal control law (7.2.2) cannot be implemented, as its parameter vectors $\boldsymbol{\theta}_{i}^{*}(t), i=1,2,20,3$, cannot be computed from the matching equations (7.2.5). In an adaptive control design, their estimates, generated from an adaptive law, are used for control implementation. Although at this point, an attractive adaptive controller structure appears to be (7.2.2) with the (unknown) nominal parameters replaced by their estimates, it will inevitably introduce "swapping" terms when we want to obtain an estimation error model for adaptive law design, by expressing an appropriately defined estimation error signal into the desired inner product of the parameter error vector and a 
regressor vector. The adaptive controller structure used in [11] cannot circumvent this issue, either. With the definition of the indicator functions (7.1.2) and to avoid introducing extra uncertainties in the estimation error model used for adaptive law design, the nominal control law is reparameterized, and a new adaptive controller structure is proposed as follows.

Reparameterization of the Nominal Control Law. By employing the nominal controller parameter vectors in terms of indicator functions as in (7.2.4), we can rewrite the nominal control law (7.2.1) as follows:

$$
\begin{aligned}
u(t) & =\sum_{i=1}^{l} \boldsymbol{\phi}_{i}^{* \top} \boldsymbol{\omega}_{i}(t) \\
\boldsymbol{\phi}_{i}^{*} & =\left[\boldsymbol{\theta}_{1 i}^{* \top}, \boldsymbol{\theta}_{2 i}^{* \top}, \theta_{20 i}^{*}, \theta_{3 i}^{*}\right]^{\top}, \quad \boldsymbol{\omega}_{i}(t)=\left[\boldsymbol{\omega}_{1 i}^{\top}(t), \boldsymbol{\omega}_{2 i}^{\top}(t), \chi_{i}(t) y(t), \chi_{i}(t) r(t)\right]^{\top}
\end{aligned}
$$

with a little abuse of notation for $\boldsymbol{\omega}_{i}(t), i \in \mathcal{I}\left(\right.$ cf. $\boldsymbol{\omega}_{j}(t), j=1,2$, in (7.2.2)), where $\boldsymbol{\omega}_{1 i}(t)=\frac{\boldsymbol{a}(s)}{\Lambda(s)}\left[\chi_{i} u\right](t)$ and $\boldsymbol{\omega}_{2 i}(t)=\frac{\boldsymbol{a}(s)}{\Lambda(s)}\left[\chi_{i} y\right](t)$ can be generated in a similar way as (7.2.2), e.g.,

$$
\begin{array}{ll}
\dot{\boldsymbol{\omega}}_{1 i}(t)=\boldsymbol{A}_{\lambda} \boldsymbol{\omega}_{1 i}(t)+\boldsymbol{b}_{\lambda} \chi_{i}(t) u(t), & \boldsymbol{\omega}_{1 i}\left(t_{0}\right)=\mathbf{0} \\
\dot{\boldsymbol{\omega}}_{2 i}(t)=\boldsymbol{A}_{\lambda} \boldsymbol{\omega}_{2 i}(t)+\boldsymbol{b}_{\lambda} \chi_{i}(t) y(t), \quad \boldsymbol{\omega}_{2 i}\left(t_{0}\right)=\mathbf{0}
\end{array}
$$

where $\left(s \boldsymbol{I}_{n}-\boldsymbol{A}_{\lambda}\right)^{-1} \boldsymbol{b}_{\lambda}=\boldsymbol{a}(s) / \Lambda(s)$ with $\boldsymbol{A}_{\lambda}$ and $\boldsymbol{b}_{\lambda}$ defined as in (7.2.3).

Adaptive Controller Structure. We replace the nominal controller parameter vectors $\phi_{i}^{*}$ of (7.3.1) by their estimates $\phi_{i}(t)$ and propose the adaptive controller structure as follows:

$$
u(t)=\sum_{i=1}^{l} \boldsymbol{\phi}_{i}^{\top}(t) \boldsymbol{\omega}_{i}(t), \quad \boldsymbol{\phi}_{i}(t)=\left[\boldsymbol{\theta}_{1 i}^{\top}(t), \boldsymbol{\theta}_{2 i}^{\top}(t), \theta_{20 i}(t), \theta_{3 i}(t)\right]^{\top}
$$

where for $i \in \mathcal{I}, \boldsymbol{\theta}_{1 i}(t), \boldsymbol{\theta}_{2 i}(t), \theta_{20 i}(t), \theta_{3 i}(t)$ are the estimates of $\boldsymbol{\theta}_{1 i}^{*}, \boldsymbol{\theta}_{2 i}^{*}, \theta_{20 i}^{*}, \theta_{3 i}^{*}$, respectively, to be updated by some adaptive laws. 


\subsubsection{Adaptive Law Design}

For the design of adaptive laws to update the controller parameters, an error model is needed. We first note that with (7.3.2) in (7.1.1) and noting the piecewise matching equation (7.2.5), the output tracking error $e(t)$ is

$$
e(t)=W_{m}(s)\left[\frac{1}{\theta_{3}^{*}} \sum_{i=1}^{l} \tilde{\boldsymbol{\phi}}_{i}^{\top} \boldsymbol{\omega}_{i}\right](t)+\eta(t)+\varepsilon_{0}(t)
$$

where $\tilde{\boldsymbol{\phi}}_{i}(t)=\phi_{i}(t)-\boldsymbol{\phi}_{i}^{*}, \varepsilon_{0}(t)$ is an initial condition related term, and $\eta(t)$ is a sum of $k-1$ terms (for $t \in\left[t_{k-1}, t_{k}\right)$ ), each determined by the internal closed-loop system state at $t_{j}, j=1,2, \ldots, k-1$.

The presence of $\eta(t)$ is due to (7.2.5), which only ensures input/output matching in a piecewise sense. Whenever a subsystem switch occurs at $t_{j}$, the part of internal system state, contributed by input over the past switching time interval $\left[t_{j-1}, t_{j}\right)$, is not matched by $y_{m}(t)$ at the system output $y(t)$. This mismatch appears as a new term in $\eta(t)$ for $t \geq t_{j}$. Neither $\eta(t)$ nor $\varepsilon_{0}(t)$ is parameterizable, and both are treated as perturbations to the parameterized part of $e(t): W_{m}(s)\left[\frac{1}{\theta_{3}^{*}} \sum_{i=1}^{l} \tilde{\boldsymbol{\phi}}_{i}^{\top} \boldsymbol{\omega}_{i}\right](t)$.

Error Model. For the the derivation of an error model suitable for adaptive law design, and considering the fact that $\theta_{3}^{*}(t)=\sum_{i=1}^{l} \theta_{3 i}^{*} \chi_{i}(t)$ and $\theta_{3 i}^{*}=1 / k_{p i}$, the output tracking error $e(t)$ in (7.3.3) can be further expressed as

$$
e(t)=\frac{1}{\theta_{3}^{*}(t)} W_{m}(s)\left[\sum_{i=1}^{l} \tilde{\boldsymbol{\phi}}_{i}^{\top} \boldsymbol{\omega}_{i}\right](t)+\eta_{1}(t)+\eta(t)+\varepsilon_{0}(t)
$$

where $\eta_{1}(t)$ is a swapping term, i.e.,

$$
\begin{aligned}
\eta_{1}(t) & =W_{m}(s)\left[\frac{1}{\theta_{3}^{*}} \sum_{i=1}^{l} \tilde{\boldsymbol{\phi}}_{i}^{\top} \boldsymbol{\omega}_{i}\right](t)-\frac{1}{\theta_{3}^{*}(t)} W_{m}(s)\left[\sum_{i=1}^{l} \tilde{\boldsymbol{\phi}}_{i}^{\top} \boldsymbol{\omega}_{i}\right](t) \\
& =\sum_{t_{j} \leq t} \boldsymbol{c}_{m}^{\top} \int_{t_{0}}^{t_{j}} e^{\boldsymbol{A}_{m}(t-\tau)} \boldsymbol{b}_{m} \sum_{i=1}^{l} \tilde{\boldsymbol{\phi}}_{i}^{\top}(\tau) \boldsymbol{\omega}_{i}(\tau) d \tau\left(\frac{1}{\theta_{3\left(t_{j-1}\right)}^{*}}-\frac{1}{\theta_{3\left(t_{j}\right)}^{*}}\right)
\end{aligned}
$$


Here, $\left(\boldsymbol{c}_{m}^{\top}, \boldsymbol{A}_{m}, \boldsymbol{b}_{m}\right)$ is a minimal state space realization of $W_{m}(s)$, i.e.,

$$
\boldsymbol{c}_{m}^{\top}\left(s \boldsymbol{I}-\boldsymbol{A}_{m}\right)^{-1} \boldsymbol{b}_{m}=W_{m}(s)
$$

and $\theta_{3\left(t_{j}\right)}^{*} \in\left\{\theta_{3 i}^{*}\right\}_{i=1}^{l}$ denotes the constant value $\theta_{3}^{*}(t)$ assumes over the switching time interval $\left[t_{j}, t_{j+1}\right)$. A key property of $\eta_{1}(t)$ is that with the controller parameter estimates generated by parameter projection adaptive laws (developed next), a normalized version of $\eta_{1}(t)$ is small in the mean square sense, which is critical to closed-loop system stability analysis.

It follows that

$$
\begin{gathered}
\theta_{3}^{*}(t) e(t)=W_{m}(s)\left[\sum_{i=1}^{l} \tilde{\boldsymbol{\phi}}_{i}^{\top} \boldsymbol{\omega}_{i}\right](t)+\theta_{3}^{*}(t)\left(\eta(t)+\varepsilon_{0}(t)+\eta_{1}(t)\right) \\
\sum_{i=1}^{l} \theta_{3 i}^{*} \chi_{i}(t) e(t)=W_{m}(s)\left[\sum_{i=1}^{l} \tilde{\boldsymbol{\phi}}_{i}^{\top} \boldsymbol{\omega}_{i}\right](t)+\theta_{3}^{*}(t)\left(\eta(t)+\varepsilon_{0}(t)+\eta_{1}(t)\right)
\end{gathered}
$$

By treating $d(t) \triangleq \theta_{3}^{*}(t)\left(\eta(t)+\varepsilon_{0}(t)+\eta_{1}(t)\right)$ as unmodeled dynamics, and defining an estimation error signal

$$
\begin{aligned}
\epsilon(t) & =\sum_{i=1}^{l}\left(\theta_{3 i}(t) \chi_{i}(t) e(t)+\xi_{i}(t)\right) \\
\xi_{i}(t) & =\boldsymbol{\phi}_{i}^{\top}(t) \boldsymbol{\zeta}_{i}(t)-W_{m}(s)\left[\boldsymbol{\phi}_{i}^{\top} \boldsymbol{\omega}_{i}\right](t), \quad \boldsymbol{\zeta}_{i}(t)=W_{m}(s)\left[\boldsymbol{\omega}_{i}\right](t)
\end{aligned}
$$

we have

$$
\begin{aligned}
\epsilon(t) & =\sum_{i=1}^{l}\left(\tilde{\theta}_{3 i}(t) \chi_{i}(t) e(t)+\tilde{\boldsymbol{\phi}}_{i}^{\top}(t) \boldsymbol{\zeta}_{i}(t)\right)+d(t) \\
\tilde{\theta}_{3 i}(t) & =\theta_{3 i}(t)-\theta_{3 i}^{*}, \quad \tilde{\boldsymbol{\phi}}_{i}(t)=\boldsymbol{\phi}_{i}(t)-\boldsymbol{\phi}_{i}^{*}
\end{aligned}
$$

The estimation error model (7.3.4) is in a form suitable for adaptive law design to update the controller parameters.

Adaptive Laws. In the presence of the unmodeled dynamics $d(t)$, we propose the following parameter projection adaptive laws to update $\phi_{i}(t), \theta_{3 i}(t), i \in \mathcal{I}$, for 
the implementation of the adaptive controller (7.3.2):

$$
\begin{aligned}
& \dot{\phi}_{i}(t)=-\frac{\boldsymbol{\Gamma}_{i} \boldsymbol{\zeta}_{i}(t) \epsilon(t)}{m^{2}(t)}+\boldsymbol{f}_{i}(t) \\
& \dot{\theta}_{3 i}(t)=-\frac{\gamma_{i} \chi_{i}(t) e(t) \epsilon(t)}{m^{2}(t)}+g_{i}(t)
\end{aligned}
$$

where $\boldsymbol{\Gamma}_{\boldsymbol{i}}>\mathbf{0}$ is diagonal, $\gamma_{i}>0$, and $\boldsymbol{f}_{i}(t)$ and $g_{i}(t)$ are parameter projection terms so designed as to confine the parameter estimates within some bounds assumed to be known. The initial estimates $\phi_{i}\left(t_{0}\right)=\phi_{i 0}$ and $\theta_{3 i}\left(t_{0}\right)=\theta_{3 i 0}$ are chosen to be within these bounds. The normalizing signal is $m^{2}(t)=1+m_{s}(t)$ with $m_{s}(t)$ generated from

$$
\dot{m}_{s}(t)=-2 \delta_{0} m_{s}(t)+u^{2}(t)+y^{2}(t), \quad m_{s}(0)=0
$$

where $\delta_{0}<\alpha$ for $\alpha$ in Assumption (A7.1-2).

\subsubsection{Stability Properties}

The proposed adaptive control scheme has the following stability properties:

Theorem 7.3.1. Consider the closed-loop system consisting of the piecewise linear system (7.1.1), the reference model system (7.1.4), and the adaptive control law (7.3.2) with parameters updated from the adaptive laws (7.3.5). There exists $\mu_{0}>0$ such that $\forall \mu \in\left[0, \mu_{0}\right)$, all signals in the closed-loop system are bounded, and

$$
\int_{t}^{t+T} e^{2}(\tau) d \tau \leq C_{2}+K_{2} \mu T, \quad \forall t \geq t_{0}, \quad \forall T>0
$$

for some $C_{2}, K_{2}>0$.

The proof of Theorem 7.3.1 can be conducted in two main steps. In the first step, some desired properties of the parameter estimates from the adaptive laws (7.3.5) are established; that is, $\phi_{i}(t), \theta_{3 i}(t), \dot{\phi}_{i}(t)$, and $\dot{\theta}_{3 i}(t)$ are bounded and within their respective bounds, and the normalized estimation error $\epsilon(t) / m(t)$ is $\mu$-small in the 
mean square sense, for as long as the truncated plant input and output signals $u(t)$ and $y(t)$ are bounded under sufficiently slow subsystem switches (characterized by all $\mu$ such that $\mu \in\left[0, \mu_{0}\right)$ for some $\left.\mu_{0}>0\right)$. This can be proved by considering the closedloop system inside each switching time interval $\left[t_{k-1}, t_{k}\right)$ as in the state space form $\left(\boldsymbol{c}_{c i}^{\top}, \boldsymbol{A}_{c i}, \boldsymbol{b}_{c i}\right)$ (see the proof of Theorem 7.2.1) with reference input $r(t)$ and an input perturbation $\frac{1}{\theta_{3 i}^{*}} \tilde{\boldsymbol{\phi}}_{i}(t) \boldsymbol{\omega}_{i}(t)$ due to controller parameter uncertainties. It can be shown that for sufficiently slow subsystem switches and provided that the truncated $u(t)$ and $y(t)$ are bounded, the plant state $\boldsymbol{x}(t)$ is bounded by the normalizing signal $m(t)$, so are the regressor vector signals $\boldsymbol{\omega}_{i}(t)$, since they are obtained by a stable filtering of $u(t)$ and $y(t)$. For the small-in-the-mean-square property of $\epsilon(t) / m(t)$, we consider $V=\tilde{\boldsymbol{\phi}}_{i}^{\top}(t) \boldsymbol{\Gamma}_{i}^{-1} \tilde{\boldsymbol{\phi}}_{i}(t)+\gamma_{i}^{-1} \tilde{\theta}_{3 i}^{2}(t)$ and its derivative over $\left[t_{k-1}, t_{k}\right)$, and integrating over the same interval. Then by combining the integrals for these switching time intervals over an interval of interest within which $u(t)$ and $y(t)$ are bounded, such a property of $\epsilon(t) / m(t)$ can be established (the effects of the discontinuities in $V$ and $d(t)$ in $\epsilon(t)$ due to subsystem switches are both characterized by $\mu$ and the length of the integration interval).

The second step is to establish closed-loop signal boundedness, $\forall t \geq t_{0}$, and to evaluate the tracking performance, based on the signal properties derived in the first step. A fictitious signal $m_{f}(t)$ is first defined in a similar way as $m_{2}(t)$ with $m_{f}(t) \geq$ $m_{2}(t)$ over the interval $\left[t_{k-1}, t_{k}\right)$, and the various signals in the closed-loop system, when normalized by $m_{f}(t)$, are either bounded or small in the mean square sense. The key part is then to establish the boundedness of $m_{f}(t)$. For such a purpose, the system I/O vector $[u(t), y(t)]^{\top}$ is expressed naturally as the output of an $\mathcal{L}^{2, \delta}$-stable operator on $r(t)+\tilde{r}(t)$, perturbed by a term dependent on the closed-loop system state at $t_{k-1}$. Such an expression can then be used, with operator inversion lemma and the 
definitions of the estimation error $\epsilon(t)$, to write the closed-loop system in a feedback framework suitable for the application of small gain arguments. In particular, the closed-loop system is expressed, over each switching time interval, as an exponentially

stable system perturbed by outputs of operators of small $\mathcal{L}^{2, \delta}$ gain, signals that have small normalized mean square values, and a signal that appears due to the subsystem switch at $t_{k-1}$ and whose normalized version is bounded by an exponentially decaying signal. Under sufficiently slow subsystem switches $\left(\mu \in\left[0, \mu_{0}\right)\right)$, the last term is small in the mean square sense, and the system loop gain is small, such that $m_{f}(t)$ is bounded, and closed-loop signal boundedness can be concluded.

\subsection{Advantages over Conventional MRAC}

If a conventional SISO output feedback for output tracking MRAC design were to be applied to the piecewise linear system (7.1.1), the controller structure will be in the following form

$$
\begin{aligned}
u(t) & =\boldsymbol{\theta}^{\boldsymbol{\top}}(t) \boldsymbol{\omega}(t) \\
\boldsymbol{\theta}(t) & =\left[\boldsymbol{\theta}_{1}^{\boldsymbol{\top}}(t), \boldsymbol{\theta}_{2}^{\boldsymbol{\top}}(t), \theta_{20}(t), \theta_{3}(t)\right]^{\boldsymbol{\top}}, \quad \boldsymbol{\omega}(t)=\left[\frac{\boldsymbol{a}^{\boldsymbol{\top}}(s)}{\Lambda(s)}[u](t) \frac{\boldsymbol{a}^{\boldsymbol{\top}}(s)}{\Lambda(s)}[y](t), y(t), r(t)\right]^{\boldsymbol{\top}}
\end{aligned}
$$

where $\boldsymbol{a}(s)$ and $\Lambda(s)$ are defined as in (7.2.1). Here, as estimates of the nominal controller parameters $\boldsymbol{\theta}_{1}^{*}(t), \boldsymbol{\theta}_{2}^{*}(t), \theta_{20}^{*}(t), \theta_{3}^{*}(t)$ defined in $(7.2 .4), \boldsymbol{\theta}_{1}(t), \boldsymbol{\theta}_{2}(t), \theta_{20}(t)$, $\theta_{3}(t)$ are directly updated by some adaptive laws.

Since the nominal controller parameter vector $\boldsymbol{\theta}^{*}(t) \triangleq\left[\boldsymbol{\theta}_{1}^{* \top}(t), \boldsymbol{\theta}_{2}^{* \top}(t), \theta_{20}^{*}(t), \theta_{3}^{*}(t)\right]^{\top}$ is time-varying (piecewise constant), and only one set of controller parameter estimates $\boldsymbol{\theta}(t) \triangleq\left[\boldsymbol{\theta}_{1}^{\boldsymbol{\top}}(t), \boldsymbol{\theta}_{2}^{\boldsymbol{\top}}(t), \theta_{20}(t), \theta_{3}(t)\right]^{\boldsymbol{\top}}$ is updated for control implementation, additional modeling error will be introduced in the error model, compared with the proposed scheme in Section 7.2. To be specific, the tracking error with the applica- 
tion of such a control law is

$$
e(t)=W_{m}(s)\left[\frac{1}{\theta_{3}^{*}} \tilde{\boldsymbol{\theta}}^{\top} \boldsymbol{\omega}\right](t)+e_{s}(t)+\eta_{s}(t)+\eta(t)+\eta_{0}(t)+\varepsilon_{0}(t)
$$

where $\tilde{\boldsymbol{\theta}}(t)=\boldsymbol{\theta}(t)-\boldsymbol{\theta}^{*}(t), \eta_{0}(t)$ and $\varepsilon_{0}(t)$ are as in (7.2.9) and (7.2.8), $e_{s}(t)$ and $\eta_{s}(t)$ are contributed by a swapping term $\Delta_{s}(t)$, to parameterize the control input error signal in terms of parameter error vector, i.e.,

$$
\begin{aligned}
\Delta_{s}(t) & =\boldsymbol{\theta}_{1}^{* \mathrm{~T}}(t) \frac{\boldsymbol{a}(s)}{\Lambda(s)}[u](t)+\boldsymbol{\theta}_{2}^{* \mathrm{~T}}(t) \frac{\boldsymbol{a}(s)}{\Lambda(s)}[y](t)-\frac{\boldsymbol{a}^{\mathrm{\top}}(s)}{\Lambda(s)}\left[\boldsymbol{\theta}_{1}^{* \mathrm{~T}} u\right](t)-\frac{\boldsymbol{a}^{\mathrm{\top}}(s)}{\Lambda(s)}\left[\boldsymbol{\theta}_{2}^{* \mathrm{~T}} y\right](t) \\
e_{s}(t) & =W_{m}(s)\left[\frac{1}{\theta_{3}^{*}} \Delta_{s}\right](t)
\end{aligned}
$$

and $\eta_{s}(t), \eta(t)$ are in the form of $\eta_{0}(t)$ in (7.2.9) with $r(\tau)$ replaced by $\frac{1}{\theta_{3}^{*}(\tau)} \Delta_{s}(\tau)$, $\frac{1}{\theta_{3}^{*}(\tau)} \tilde{\boldsymbol{\theta}}^{\top}(\tau) \boldsymbol{\omega}(\tau)$, respectively.

Define the estimation error signal $\epsilon(t)=e(t)+\rho(t) \xi(t)$, where $\xi(t)=\boldsymbol{\theta}^{\top}(t) \boldsymbol{\zeta}(t)-$ $W_{m}(s)\left[\boldsymbol{\theta}^{\top} \boldsymbol{\omega}\right](t), \boldsymbol{\zeta}(t)=W_{m}(s)[\boldsymbol{\omega}](t)$, and $\rho(t)$ is an estimate of $\rho^{*}(t)=1 / \theta_{3}^{*}(t)$. It follows that

$$
\begin{aligned}
\epsilon(t)= & \rho^{*}(t) \tilde{\boldsymbol{\theta}}^{\top}(t) \boldsymbol{\zeta}(t)+\tilde{\rho}(t) \xi(t)+\varepsilon_{a}(t)+e_{s}(t)+\eta_{s}(t)+\eta(t)+\eta_{0}(t)+\varepsilon_{0}(t) \\
\varepsilon_{a}(t)= & W_{m}(s)\left[\rho^{*} \tilde{\boldsymbol{\theta}}^{\top} \boldsymbol{\omega}\right](t)-\rho^{*}(t) W_{m}(s)\left[\tilde{\boldsymbol{\theta}}^{\top} \boldsymbol{\omega}\right](t) \\
& +\rho^{*}(t)\left(\boldsymbol{\theta}^{* \boldsymbol{\top}}(t) \boldsymbol{\zeta}(t)-W_{m}(s)\left[\boldsymbol{\theta}^{* \boldsymbol{\top}} \boldsymbol{\omega}\right](t)\right)
\end{aligned}
$$

With the knowledge of lower and upper bounds on system parameters, the following parameter projection adaptive laws can be applied to update $\boldsymbol{\theta}(t)$ and $\rho(t)$ :

$$
\begin{aligned}
& \dot{\boldsymbol{\theta}}(t)=-\frac{\operatorname{sign}\left[\rho^{*}(t)\right] \boldsymbol{\Gamma} \boldsymbol{\zeta}(t) \epsilon(t)}{m^{2}(t)}+\boldsymbol{f}(t), \quad \boldsymbol{\Gamma}=\boldsymbol{\Gamma}^{\boldsymbol{\top}}>\mathbf{0} \\
& \dot{\rho}(t)=-\frac{\gamma \xi(t) \epsilon(t)}{m^{2}(t)}+g(t), \quad \gamma>0, \quad t \geq 0
\end{aligned}
$$

where the projection terms $\boldsymbol{f}(t)$ and $g(t)$ are designed to confine the parameter estimates to be within their respective bounds at all time, and $m(t)$ is the normalizing signal generated in the same way as in (7.3.6). 
To implement this design, we need to assume that $\rho^{*}(t)$ has a known and constant sign; that is, $\operatorname{sign}\left[k_{p i}\right]$ is known and constant. Then with sufficient slow subsystem switches, closed-loop stability can be established.

The major advantage of the proposed adaptive control scheme over this conventional MRAC design is the effective use of the knowledge of the indicator functions to improve the parameterization of the estimation error signal for reduced unmodeled dynamics. It is obvious that extra unmoldeled dynamics terms $\varepsilon_{a}(t), e_{s}(s)$, and $\eta_{s}(t)$, due to swapping, exist in (7.4.2), as compared with (7.3.4). Furthermore, for control implementation, $\operatorname{sign}\left[k_{p i}\right]$ of each system model is not required to be the same.

\subsection{Simulation Study}

In this section a simulation study is presented to demonstrate the system stability and tracking performance with the proposed adaptive control scheme applied to the piecewise linear system model of the longitudinal dynamics of the NASA GTM [82] at multiple operating points.

\section{Linearized Aircraft Longitudinal Model and Reference Model System.}

For simplicity of presentation, we choose $l=2$, and trim the GTM at steady-state, straight, wings-level flight condition at 80 knots and 90 knots at $800 \mathrm{ft}$., respectively, to obtain a piecewise linear longitudinal system model in the form of (7.1.1), where $\boldsymbol{x}=[u, w, q, \theta]^{\top}$ with the elements being the perturbed aircraft velocity components along the $\mathrm{x}$ - and z-body-axis (fps), angular velocity along the y-body-axis ( $\mathrm{rad} / \mathrm{s})$, and pitch angle (rad), respectively. The control input is the perturbed elevator deflection 
$\delta_{e}$, and the parameter matrices are

$$
\begin{aligned}
\boldsymbol{A}_{1}= & {\left[\begin{array}{rrrr}
-0.0293 & 0.2460 & -8.9936 & -32.0974 \\
-0.2611 & -3.0403 & 129.7255 & -2.2189 \\
0.0175 & -0.3202 & -3.8364 & 0 \\
0 & 0 & 1.0000 & 0
\end{array}\right] } \\
\boldsymbol{A}_{2}= & {\left[\begin{array}{rrrr}
-0.0380 & 0.2786 & -7.5029 & -32.1318 \\
-0.2440 & -3.4119 & 146.2253 & -1.6482 \\
0.0136 & -0.3581 & -4.4019 & 0 \\
0 & 0 & 1.0000 & 0
\end{array}\right] } \\
\boldsymbol{b}_{1}= & {\left[\begin{array}{r}
-0.0010 \\
-0.0031 \\
-0.6953 \\
-0.8526 \\
0
\end{array}\right], \boldsymbol{b}_{2}=\left[\begin{array}{l}
0 \\
-1.0866 \\
0
\end{array}\right], \boldsymbol{c}=\left[\begin{array}{l}
0 \\
1
\end{array}\right] }
\end{aligned}
$$

with the corresponding transfer functions:

$$
\begin{aligned}
& G_{1}(s)=\frac{-85.26 s^{2}-239.4 s-12.66}{s^{4}+6.906 s^{3}+53.62 s^{2}+2.324 s+4.375} \\
& G_{2}(s)=\frac{-108.7 s^{2}-343.7 s-20.63}{s^{4}+7.852 s^{3}+67.85 s^{2}+3.157 s+4.286}
\end{aligned}
$$

where we have chosen the pitch angle $\theta$ as the output, i.e., $y=\theta$. It can be verified that all design assumptions are satisfied: i) system zeros for $G_{1}(s)$ are $z_{11,12}=$ $-2.7544,-0.0539$, and those for $G_{2}(s)$ are $z_{21,22}=-3.1019,-0.0612$, so that $\delta_{0}$ in (7.3.6) can be chosen as $\delta_{0}=0.05$; ii) for $i=1,2,\left(\boldsymbol{c}^{\top}, \boldsymbol{A}_{i}\right)$ is observable and $\left(\boldsymbol{A}_{i}, \boldsymbol{b}_{i}\right)$ is controllable; iii) $n=2$ and $m=2$, thus $n^{*}=n-m=2$; and iv) $\operatorname{sign}\left[k_{p 1}\right]=\operatorname{sign}\left[k_{p 2}\right]=-1$. The reference model system transfer function is thus chosen as

$$
W_{m}(s)=\frac{1}{(s+1)(s+2)}
$$

Matching Condition and Nominal Parameters. It can be verified that the piecewise plant-model matching equation (7.2.5) is satisfied with the following nomi- 
nal controller parameter vectors:

$$
\begin{aligned}
& \boldsymbol{\theta}_{11}^{*}=[0.5936,2.1631,0.4073]^{\top}, \quad \theta_{201}=5.2687 \\
& \boldsymbol{\theta}_{21}^{*}=[-2.3997,1.0295,0.6537]^{\top}, \quad \theta_{31}=-1.1729 \\
& \boldsymbol{\theta}_{12}^{*}=[0.3042,1.9939,-0.1729]^{\top}, \quad \theta_{202}=4.3689 \\
& \boldsymbol{\theta}_{22}^{*}=[-1.5750,0.6965,0.4984]^{\top}, \quad \theta_{32}=-0.9200
\end{aligned}
$$

Simulation Results. In this simulation, we choose a switching time interval $T=400 \mathrm{~s}$. The initial plant state is $[5,2,0,0.1]^{\top}$ with zero reference model initial condition, and the initial parameter estimates are chosen to be $90 \%$ of their nominal values. The adaptation gains are $\boldsymbol{\Gamma}_{i}=150$, and $\gamma_{i}=100, i=1,2$.

Figure 7.1 shows the output tracking performance with a sinusoidal reference input $r(t)=0.2 \sin (\pi t / 200)$, corresponding to a desired fluctuation of the pitch angle in between $\pm 5.73^{\circ}$. The elevator deflection $\delta_{e}(t)$ is shown at the bottom of Figure 7.1. As a comparison, the tracking performance with the conventional SISO output feedback for output tracking MRAC scheme under the same conditions is plotted in Fig. 7.2, together with $\delta_{e}(t)$.

Simulations are performed under the same conditions for a constant reference input $r(t)=0.2$, corresponding to a desired constant pitch angle of $5.73^{\circ}$. The tracking performance is plotted in Figures 7.3 and 7.4, respectively, for the proposed scheme and the conventional MRAC scheme.

From the simulation results, we can see that the elevator deflections are within reasonable limits $\left( \pm 20^{\circ}\right)$, and closed-loop stability is achieved for both cases. However, there is an obvious improvement in output tracking performance by the proposed adaptive control scheme, as compared with the conventional MRAC scheme under the same conditions.

In this chapter, an output feedback MRAC scheme is presented for SISO piecewise 

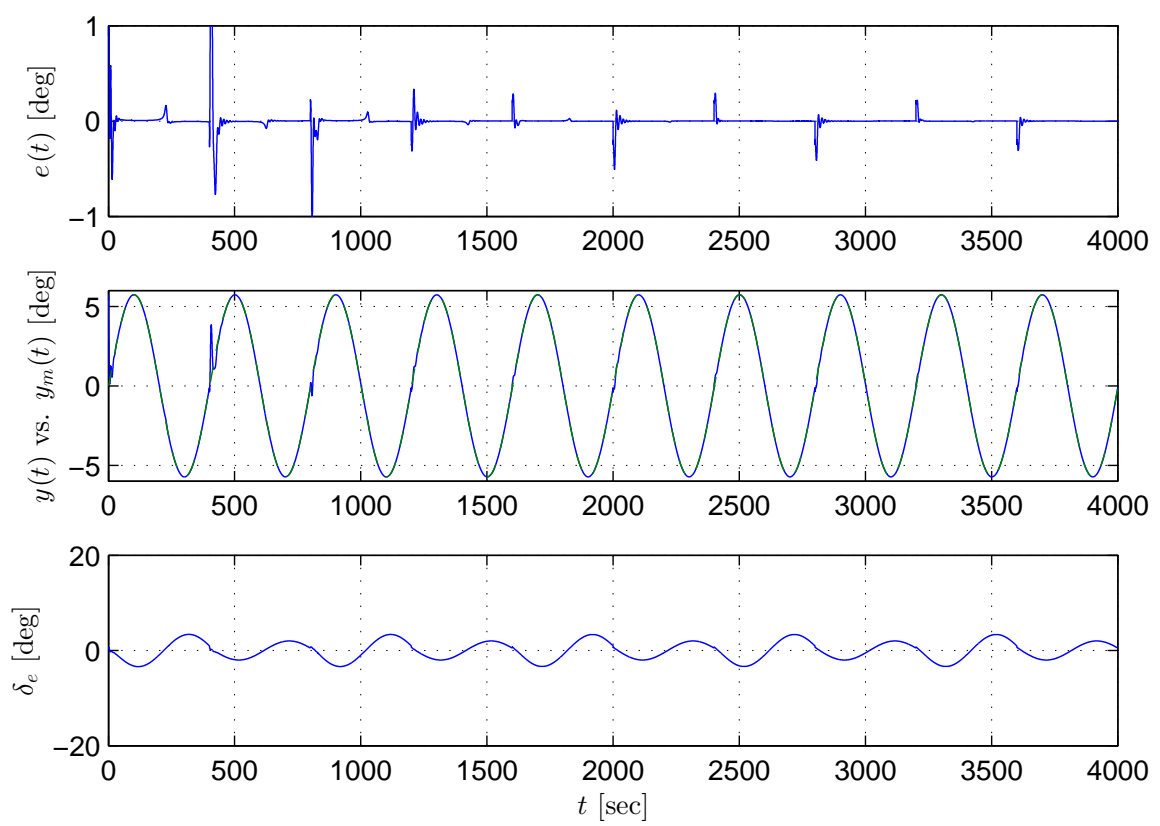

Figure 7.1: Output tracking performance and the control input $\delta_{e}$ with $r(t)=$ $0.2 \sin (\pi t / 200)$ : the proposed scheme.
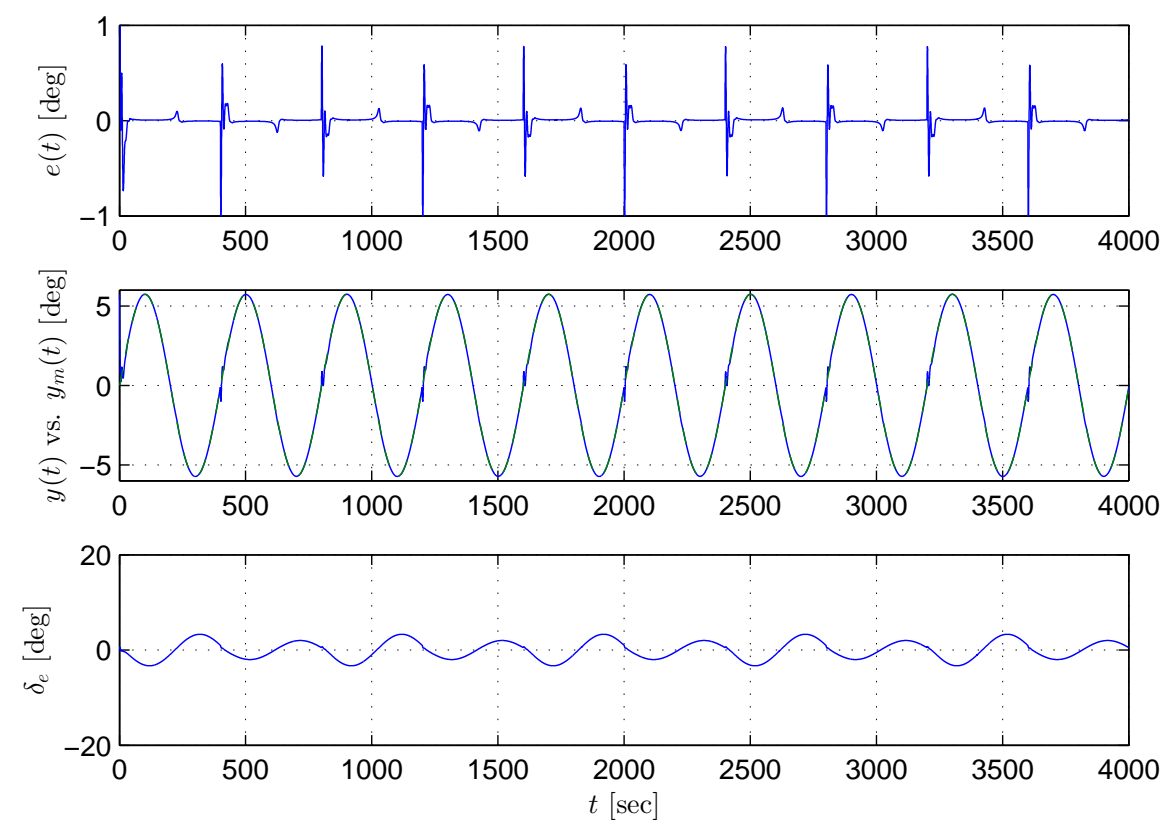

Figure 7.2: Output tracking performance and the control input $\delta_{e}$ with $r(t)=$ $0.2 \sin (\pi t / 200)$ : the conventional MRAC scheme. 

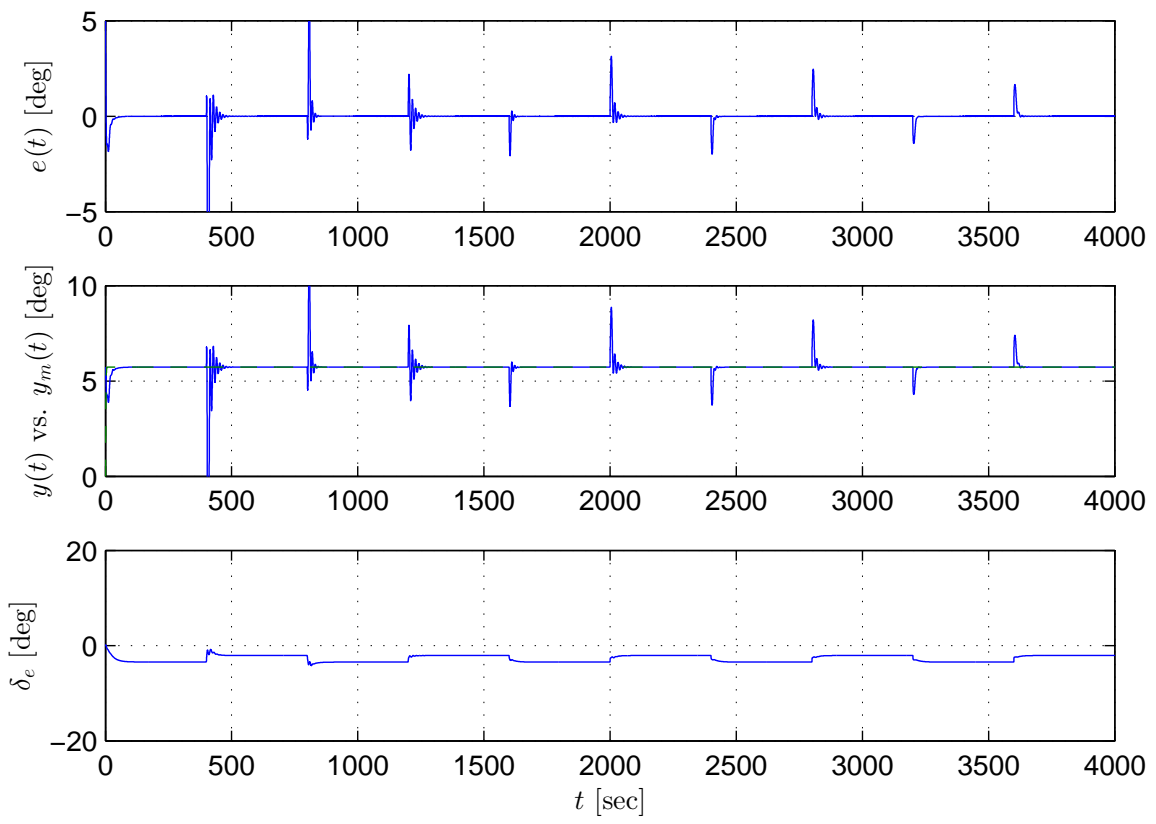

Figure 7.3: Output tracking performance and the control input $\delta_{e}$ with $r(t)=0.2$ : the proposed scheme.
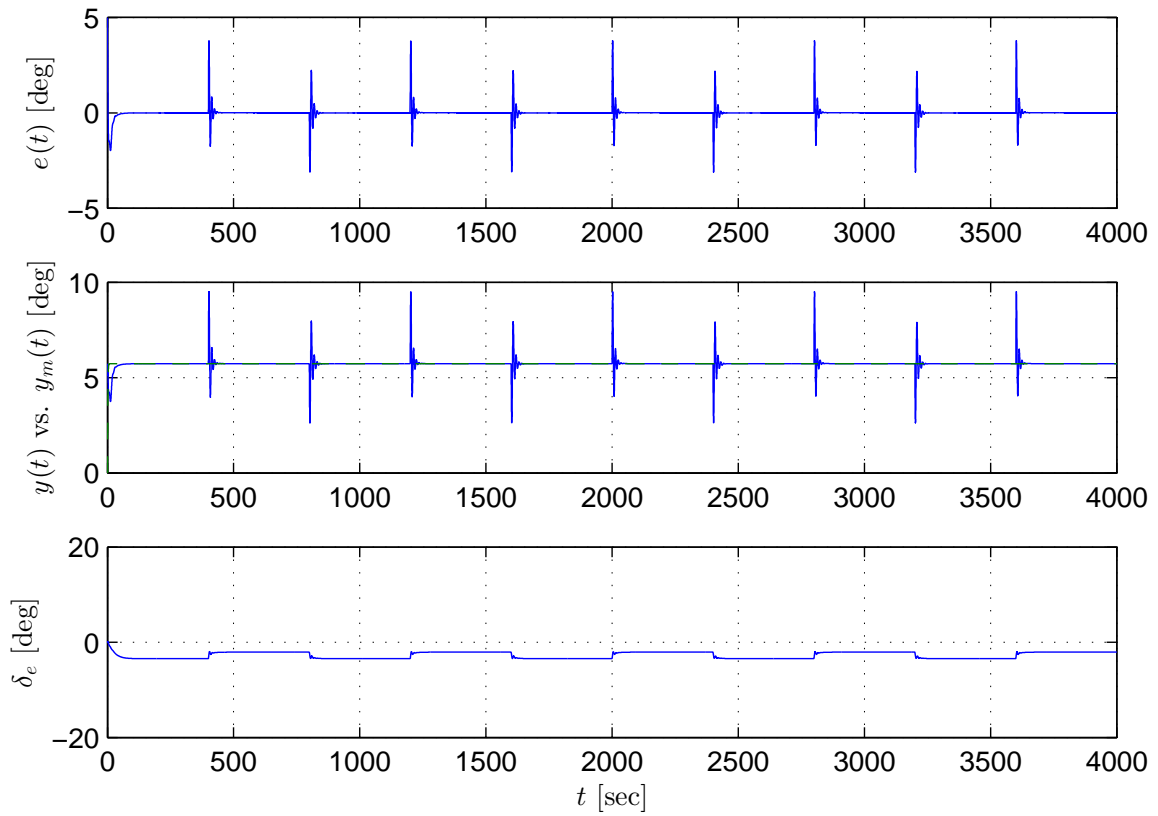

Figure 7.4: Output tracking performance and the control input $\delta_{e}$ with $r(t)=0.2$ : the conventional MRAC scheme. 
linear systems. The results presented in this chapter rely on the analysis method used in $[11,57]$, where the authors generalized their previous results in [83] to include parameter discontinuities and time variations inside intervals of continuity (the latter not considered in the context of piecewise linear systems) through robustness analysis. It is shown that with the proposed MRAC scheme, closed-loop stability and smallin-the-mean-square output tracking performance are achieved, if the frequency of parameter discontinuities is sufficiently low on average. The contribution of this chapter is a reparametrization of the model reference controller structure [83] and the proposal of a new adaptive control design. A feature of our design is the avoidance of additional modeling errors in the closed-loop system due to controller parameter swapping. The price paid is the assumption of the knowledge of the time instants at which plant parameters switch. This is not a restrictive assumption in many practical applications. For example, for an aircraft in a typical straight, wings-level, steadystate flight condition, its velocity and altitude can be used as an indicator of operating points. 


\section{Chapter 8}

\section{Conclusions}

Adaptive control, which automatically updates controller parameters by adaptive laws to accommodate system uncertainties, is an attractive and promising approach to the design of control systems for performance-critical systems, such as aircraft flight

control systems. For adaptive control systems, a suitable stability and performance metric such as gain margin is desirable in evaluating and comparing adaptive controllers and maturing adaptive control designs for implementation on performancecritical systems. On the other hand, adaptive control designs for piecewise linear systems are expected to be capable of expanding nonlinear system operating range in the presence of uncertainties. This dissertation focuses on the gain margin (GM) of adaptive control systems and the development of novel adaptive control schemes for piecewise linear systems. The contributions of this dissertation are a systematic gain margin analysis for commonly used adaptive control designs, and a framework of solutions to the open problems in adaptive control of uncertain piecewise linear systems.

The work presented in this dissertation is summarized as follows:

- The basic problems considered in this dissertation are formulated in Chapter 2, including the gain margins of adaptive control systems, the performance robust- 
ness issue of model reference adaptive control (MRAC) systems with respect to reduced actuator effectiveness, and adaptive control of piecewise linear systems.

- In Chapter 3, the gain margin problem has been studied for various MRAC systems: those with direct or indirect, continuous-time or discrete-time designs. The analytical gain margin results are summarized in Table 3.1. These GM results are also applicable to other adaptive control systems such as adaptive state feedback control systems and adaptive nonlinear control systems.

- Based on the derived analytical gain margin results in Chapter 3, MRAC schemes are studied for their inherent performance robustness properties with regard to reduced actuator effectiveness in Chapter 4.

- The theoretical gain margin results and performance robustness properties of MRAC have been demonstrated by simulation studies.

- The adaptive control problem is studied for piecewise linear systems.

- In Chapter 5, adaptive state feedback schemes are designed for piecewise linear systems to achieve state tracking performance. The reference trajectory is specified by a piecewise linear reference model system, and its stability properties are investigated.

- Adaptive state feedback schemes are designed for piecewise linear systems to achieve output tracking in Chapter 6 .

- Adaptive output feedback schemes are designed for piecewise linear systems to achieve output tracking in Chapter 7.

- Key issues to adaptive control of piecewise linear systems, such as controller 
structure, matching conditions, error models, adaptive laws, closed-loop stability and tracking performance evaluation, have been investigated.

- The proposed adaptive control designs have been applied to some aircraft system models with simulation results to demonstrate their effectiveness in tracking performance improvement.

As future research topics in this area, the results reported in this dissertation can be extended in a few directions.

- In the multivariable state feedback for output tracking design for piecewise linear systems (Section 6.2), Assumption (A6.2-5) is made; that is, the high frequency gain matrix $\boldsymbol{K}_{p i}$ of each subsystem is assumed to have a common $\boldsymbol{L}_{s}$ matrix in their LDS decompositions. Such an assumption facilitates the derivation of an error model to avoid differentiation operations on the tracking error signal. The relaxation of this assumption is desirable for practical implementation of the proposed design. The task is to study the properties of a swapping term introduced due to the difference in $\boldsymbol{L}_{s}$ matrices for each subsystem and its impact on closed-loop stability and tracking performance.

- The successful relaxation of Assumption (A6.2-5) is also critical for extending the output feedback for output tracking design presented in Chapter 7 to handle multi-input, multi-output (MIMO) controlled systems, based on high frequency gain matrix decompositions.

- In the proposed adaptive control designs, indicator functions are assumed to be known; that is, subsystem switches are not known a priori, but assumed to be detected instantaneously. It is interesting to investigate the system performance 
robustness with regard to delay in subsystem switch detection. In particular, supposing that subsystem switches can be detected $\tau$ units of time after the switching time instant $t_{k}$, whether or not and to what extent closed-loop stability and tracking performance are still achievable are of interest.

- In this dissertation, the attention is focused on piecewise linear systems. Feedback linearization and backstepping have been powerful tools in adaptive nonlinear control. How to effectively employ these tools in the development of adaptive control designs for piecewise nonlinear systems is of major interest. 


\section{Chapter 9}

\section{Appendix}

\subsection{Derivations of (3.4.15)-(3.4.18)}

This section contains a guideline for the choice of $\gamma_{1}$ and $\gamma_{2}$ in the discrete-time MIMO direct MRAC design for $M=2$, based on which an explicit gain margin result is derived in Section 3.4.3.

When the constant gain matrix $\boldsymbol{K}=\boldsymbol{I}_{2}$, we have

$$
\boldsymbol{D}^{*}=\operatorname{diag}\left\{d_{1}^{*}, d_{2}^{*}\right\}=\operatorname{diag}\left\{\Delta_{1}, \frac{\Delta_{2}}{\Delta_{1}}\right\}
$$

from (2.2.10), and $\boldsymbol{D}_{s}=\operatorname{diag}\left\{\operatorname{sign}\left[d_{1}^{*}\right] \gamma_{1}, \operatorname{sign}\left[d_{2}^{*}\right] \gamma_{2}\right\}$ from (2.2.11). Substituting $\boldsymbol{D}^{*}$, $\boldsymbol{D}_{s}$, and $\boldsymbol{U}=\left[\begin{array}{ll}1 & a \\ 0 & 1\end{array}\right]$ into (3.4.8) yields

$$
\mathbf{0}<\left[\begin{array}{cc}
\left|d_{1}^{*}\right| \gamma_{1} & a d_{1}^{*} \operatorname{sign}\left[d_{2}^{*}\right] \gamma_{2} \\
a d_{1}^{*} \operatorname{sign}\left[d_{2}^{*}\right] \gamma_{2} & a^{2}\left|d_{1}^{*}\right| \frac{\gamma_{2}^{2}}{\gamma_{1}}+\left|d_{2}^{*}\right| \gamma_{2}
\end{array}\right]<2 \boldsymbol{I}_{2}
$$

The right inequality is equivalent to

$$
\left[\begin{array}{cc}
\left|d_{1}^{*}\right| \gamma_{1}-2 & a d_{1}^{*} \operatorname{sign}\left[d_{2}^{*}\right] \gamma_{2} \\
a d_{1}^{*} \operatorname{sign}\left[d_{2}^{*}\right] \gamma_{2} & a^{2}\left|d_{1}^{*}\right| \frac{\gamma_{2}^{2}}{\gamma_{1}}+\left|d_{2}^{*}\right| \gamma_{2}-2
\end{array}\right]<\mathbf{0}
$$

which requires $\gamma_{1}$ and $\gamma_{2}$ to satisfy:

$$
\left|d_{1}^{*}\right| \gamma_{1}-2<0
$$




$$
\operatorname{det}\left[\begin{array}{cc}
\left|d_{1}^{*}\right| \gamma_{1}-2 & a d_{1}^{*} \operatorname{sign}\left[d_{2}^{*}\right] \gamma_{2} \\
a d_{1}^{*} \operatorname{sign}\left[d_{2}^{*}\right] \gamma_{2} & a^{2}\left|d_{1}^{*}\right| \frac{\gamma_{2}^{2}}{\gamma_{1}}+\left|d_{2}^{*}\right| \gamma_{2}-2
\end{array}\right]>\mathbf{0}
$$

The inequality (9.1.2) is satisfied if the range for $\gamma_{1}$ as in (3.4.15) is satisfied. The inequality (9.1.3) yields

$$
\left(\left|d_{1}^{*}\right| \gamma_{1}-2\right)\left(\left|d_{2}^{*}\right| \gamma_{2}-2\right)>2 a^{2}\left|d_{1}^{*}\right| \frac{\gamma_{2}^{2}}{\gamma_{1}}
$$

which can be satisfied if

$$
\left(d_{1}^{0} \gamma_{1}-2\right)\left(d_{2}^{0} \gamma_{2}-2\right)>2 a^{2} d_{1}^{0} \frac{\gamma_{2}^{2}}{\gamma_{1}}
$$

where $d_{2}^{0}$ is the upper bound of $\left|d_{2}^{*}\right|$ assumed to be known.

After choosing a $\gamma_{1}$ satisfying (3.4.15), we have to choose a $\gamma_{2}$ satisfying (9.1.5). Note that in (9.1.5), $d_{1}^{0} \gamma_{1}-2<0$ and the right-hand-side term is nonnegative, thus $d_{2}^{0} \gamma_{2}-2$ must be negative, which requires $0<\gamma_{2}<2 / d_{2}^{0}$. Besides, (9.1.5) can be rewritten as

$$
2 a^{2} d_{1}^{0} \gamma_{2}^{2}-\gamma_{1}\left(d_{1}^{0} \gamma_{1}-2\right) d_{2}^{0} \gamma_{2}+2 \gamma_{1}\left(d_{1}^{0} \gamma_{1}-2\right)<0
$$

from which, if we let $\alpha\left(\gamma_{1}\right)=\gamma_{1}\left(d_{1}^{0} \gamma_{1}-2\right), \beta\left(\gamma_{1}\right)=\alpha\left(\gamma_{1}\right)\left(d_{2}^{0}\right)^{2}-16 a^{2} d_{1}^{0}$, and assume $a \neq 0$, we can obtain

$$
\frac{\alpha\left(\gamma_{1}\right) d_{2}^{0}-\sqrt{\alpha\left(\gamma_{1}\right) \beta\left(\gamma_{1}\right)}}{4 a^{2} d_{1}^{0}}<\gamma_{2}<\frac{\alpha\left(\gamma_{1}\right) d_{2}^{0}+\sqrt{\alpha\left(\gamma_{1}\right) \beta\left(\gamma_{1}\right)}}{4 a^{2} d_{1}^{0}}
$$

Therefore, a $\gamma_{2}$ has to be chosen to satisfy

$$
\max \left\{0, \frac{\alpha\left(\gamma_{1}\right) d_{2}^{0}-\sqrt{\alpha\left(\gamma_{1}\right) \beta\left(\gamma_{1}\right)}}{4 a^{2} d_{1}^{0}}\right\}<\gamma_{2}<\min \left\{\frac{2}{d_{2}^{0}}, \frac{\alpha\left(\gamma_{1}\right) d_{2}^{0}+\sqrt{\alpha\left(\gamma_{1}\right) \beta\left(\gamma_{1}\right)}}{4 a^{2} d_{1}^{0}}\right\}
$$

Note that $\alpha\left(\gamma_{1}\right)<0, \beta\left(\gamma_{1}\right)<0$, and $\sqrt{\alpha\left(\gamma_{1}\right) \beta\left(\gamma_{1}\right)}>-\alpha\left(\gamma_{1}\right) d_{2}^{0}$, we have

$$
\max \left\{0, \frac{\alpha\left(\gamma_{1}\right) d_{2}^{0}-\sqrt{\alpha\left(\gamma_{1}\right) \beta\left(\gamma_{1}\right)}}{4 a^{2} d_{1}^{0}}\right\}=0
$$


Furthermore, from the expressions of $\alpha\left(\gamma_{1}\right)$ and $\beta\left(\gamma_{1}\right)$, we can derive

$$
\frac{2}{d_{2}^{0}}-\frac{\alpha\left(\gamma_{1}\right) d_{2}^{0}+\sqrt{\alpha\left(\gamma_{1}\right) \beta\left(\gamma_{1}\right)}}{4 a^{2} d_{1}^{0}} \geq 0
$$

From (9.1.8), (9.1.9) and (9.1.10), we have the range of $\gamma_{2}$ for $a \neq 0$ in (3.4.16). The equal sign in (9.1.10) is satisfied if and only if $\sqrt{-\alpha\left(\gamma_{1}\right)} d_{2}^{0}=\sqrt{-\beta\left(\gamma_{1}\right)}$, which requires $a=0$, or $\boldsymbol{U}=\boldsymbol{I}_{2}$. This results in $\gamma_{1}$ and $\gamma_{2}$ satisfying (3.4.15).

When the gain matrix $\boldsymbol{K}=\operatorname{diag}\left\{k_{1}, k_{2}\right\}$ is not equal to the identity matrix $\boldsymbol{I}_{2}$, by following a similar procedure as in (9.1.1)-(9.1.4), (3.4.11) yields

$$
\begin{gathered}
\left|d_{1}^{*}\right| k_{1} \gamma_{1}-2<0 \\
\left(\left|d_{1}^{*}\right| k_{1} \gamma_{1}-2\right)\left(\left|d_{2}^{*}\right| k_{2} \gamma_{2}-2\right)>2 a^{2}\left|d_{1}^{*}\right| \frac{k_{2}^{2} \gamma_{2}^{2}}{k_{1} \gamma_{1}}
\end{gathered}
$$

where $\gamma_{1}$ satisfies (3.4.15), and $\gamma_{2}$ satisfies (3.4.15) for $\boldsymbol{U}=\boldsymbol{I}_{2}$ and (3.4.16) for $\boldsymbol{U} \neq \boldsymbol{I}_{2}$. From (3.4.15), (9.1.11) is satisfied if $0<k_{1} \leq \frac{d_{1}^{0}}{\left|d_{1}^{*}\right|}$.

Similar to the above analysis, assuming $a \neq 0$, we obtain

$$
0<k_{2} \gamma_{2}<\frac{\bar{\alpha}\left(k_{1} \gamma_{1}\right)\left|d_{2}^{*}\right|+\sqrt{\bar{\alpha}\left(k_{1} \gamma_{1}\right) \bar{\beta}\left(k_{1} \gamma_{1}\right)}}{4 a^{2}\left|d_{1}^{*}\right|}
$$

From (3.4.16), the above inequality is satisfied if

$$
0<k_{2} \frac{\alpha\left(\gamma_{1}\right) d_{2}^{0}+\sqrt{\alpha\left(\gamma_{1}\right) \beta\left(\gamma_{1}\right)}}{4 a^{2} d_{1}^{0}} \leq \frac{\bar{\alpha}\left(k_{1} \gamma_{1}\right)\left|d_{2}^{*}\right|+\sqrt{\bar{\alpha}\left(k_{1} \gamma_{1}\right) \bar{\beta}\left(k_{1} \gamma_{1}\right)}}{4 a^{2}\left|d_{1}^{*}\right|}
$$

Therefore, we have $k_{2}$ satisfying (3.4.18) for $a \neq 0$. For the case $a=0$, (9.1.11) and (9.1.12) lead to $\left|d_{2}^{*}\right| k_{2} \gamma_{2}-2<0$, which, from (3.4.15), is satisfied if $k_{2}$ satisfies $(3.4 .17)$.

\subsection{Proof of Lemma 5.2.1}

Let $\boldsymbol{A}_{m(k-1)} \in\left\{\boldsymbol{A}_{m 1}, \ldots, \boldsymbol{A}_{m l}\right\}$ denote the subsystem of $\boldsymbol{A}_{m}(t)$ over $\left[t_{k-1}, t_{k}\right), k \in \mathbb{Z}^{+}$. Consider a subsystem switch at $t=t_{k}$. Due to the stability of $\boldsymbol{A}_{m(k-1)}, \boldsymbol{A}_{m(k)}$ and 
without loss of generality, there exist symmetric, positive definite $\boldsymbol{P}_{m(k-1)}, \boldsymbol{P}_{m(k)} \in$ $\mathbb{R}^{n \times n}$ such that

$$
\begin{array}{r}
\boldsymbol{A}_{m(k-1)}^{\top} \boldsymbol{P}_{m(k-1)}+\boldsymbol{P}_{m(k-1)} \boldsymbol{A}_{m(k-1)}=-\boldsymbol{I}_{n} \\
\boldsymbol{A}_{m(k)}^{\top} \boldsymbol{P}_{m(k)}+\boldsymbol{P}_{m(k)} \boldsymbol{A}_{m(k)}=-\boldsymbol{I}_{n}
\end{array}
$$

where $\boldsymbol{I}_{n} \in \mathbb{R}^{n \times n}$ is the identity matrix. With $\Delta \boldsymbol{A}_{m(k)}=\boldsymbol{A}_{m(k)}-\boldsymbol{A}_{m(k-1)}, \Delta \boldsymbol{P}_{m(k)}=$ $\boldsymbol{P}_{m(k)}-\boldsymbol{P}_{m(k-1)}$, we have

$$
\begin{array}{r}
\boldsymbol{A}_{m(k)}^{\top} \Delta \boldsymbol{P}_{m(k)}+\Delta \boldsymbol{P}_{m(k)} \boldsymbol{A}_{m(k)}=-\boldsymbol{S}_{(k)} \\
\boldsymbol{S}_{(k)}=\Delta \boldsymbol{A}_{m(k)}^{\top} \boldsymbol{P}_{m(k-1)}+\boldsymbol{P}_{m(k-1)} \Delta \boldsymbol{A}_{m(k)}
\end{array}
$$

thus $\left\|\boldsymbol{S}_{(k)}\right\| \leq 2\left\|\boldsymbol{P}_{m(k-1)}\right\|\left\|\Delta \boldsymbol{A}_{m(k)}\right\|$. Since $\boldsymbol{A}_{m(k)}$ is stable, the solution of (9.2.1) is $\Delta \boldsymbol{P}_{m(k)}=\int_{0}^{\infty} e^{\boldsymbol{A}_{m(k)}^{\top} t} \boldsymbol{S}_{(k)} e^{\boldsymbol{A}_{m(k)} t} d t$. With $\left\|e^{\boldsymbol{A}_{m(k)} t}\right\| \leq a_{m} e^{-\lambda_{m} t}$, we have

$$
\begin{aligned}
\left\|\Delta \boldsymbol{P}_{m(k)}\right\| & \leq\left\|\boldsymbol{S}_{(k)}\right\| \int_{0}^{\infty}\left\|e^{\boldsymbol{A}_{m(k)}^{\top}}\right\|\left\|e^{\boldsymbol{A}_{m}^{k} t}\right\| d t \\
& \leq \frac{a_{m}^{2}}{\lambda_{m}}\left\|\boldsymbol{P}_{m(k-1)}\right\|\left\|\Delta \boldsymbol{A}_{m(k)}\right\|
\end{aligned}
$$

Consider the piecewise continuous Lyapunov function

$$
V=\boldsymbol{z}^{\top}(t) \sum_{i=1}^{l} \boldsymbol{P}_{m i} \chi_{i}(t) \boldsymbol{z}(t)
$$

with $\boldsymbol{P}_{m i}$ satisfying (5.2.3) for $\boldsymbol{Q}_{m i}=\boldsymbol{I}_{n}$ so that $\boldsymbol{P}_{m(k-1)}$ and $\boldsymbol{P}_{m(k)} \in\left\{\boldsymbol{P}_{m 1}, \ldots, \boldsymbol{P}_{m l}\right\}$. At $t=t_{k},(9.2 .3)$ and the fact that $\beta\|\boldsymbol{z}(t)\|^{2} \leq V$ lead to

$$
V\left(t_{k}\right)-V\left(t_{k}^{-}\right)=\boldsymbol{z}^{\top}\left(t_{k}\right) \Delta \boldsymbol{P}_{m(k)} \boldsymbol{z}\left(t_{k}\right) \leq \frac{a_{m}^{2}}{\lambda_{m} \beta}\left\|\boldsymbol{P}_{m(k-1)}\right\|\left\|\Delta \boldsymbol{A}_{m(k)}\right\| V\left(t_{k}^{-}\right)
$$

With $\mu=\frac{a_{m}^{2}}{\lambda_{m} \beta} \max _{i \in \mathcal{I}}\left\|\boldsymbol{P}_{m i}\right\|$, we have

$$
V\left(t_{k}\right) \leq\left(1+\mu \Delta_{\boldsymbol{A}_{m}}\right) V\left(t_{k}^{-}\right)
$$

In addition, with $V \leq \alpha\|\boldsymbol{z}(t)\|^{2}$, the time derivative of $V$ over $\left[t_{k-1}, t_{k}\right)$ satisfies $\dot{V} \leq-V / \alpha$, and

$$
V(t) \leq e^{-\frac{1}{\alpha}\left(t-t_{0}\right)}\left(1+\mu \Delta_{\boldsymbol{A}_{m}}\right)^{k-1} V\left(t_{0}\right), \quad t \in\left[t_{k-1}, t_{k}\right)
$$


which leads to

$$
\|\boldsymbol{z}(t)\| \leq\left(\frac{\alpha}{\beta}\right)^{\frac{1}{2}} e^{-\frac{1}{2 \alpha}\left(t-t_{0}\right)+\frac{k-1}{2} \ln \left(1+\mu \Delta_{\boldsymbol{A}_{m}}\right)}\left\|\boldsymbol{z}\left(t_{0}\right)\right\|
$$

Furthermore, with $T_{0}$ being the minimum switching time interval, we have $t-t_{0} \geq$ $(k-1) T_{0}$ for $t \in\left[t_{k-1}, t_{k}\right)$, which together with the condition (5.2.4), leads to

$$
-\frac{1}{2 \alpha}\left(t-t_{0}\right)+\frac{k-1}{2} \ln \left(1+\mu \Delta_{\boldsymbol{A}_{m}}\right) \leq-\sigma\left(t-t_{0}\right)
$$

and exponential stability can thus be concluded.

\subsection{Proof of Theorem 5.3.1}

Due to the fact $T_{d}>\alpha \ln \left(1+\mu \Delta_{\boldsymbol{A}_{m}}\right)$, it follows from Lemma 5.2.1 that $T_{0} \geq T_{d}$ ensures stability of (5.2.1), i.e., $\boldsymbol{x}_{m}(t) \in \mathcal{L}^{\infty}$. Consider the piecewise continuous Lyapunov function

$$
V=\boldsymbol{e}^{\top} \sum_{i=1}^{l} \boldsymbol{P}_{m i} \chi_{i} \boldsymbol{e}+\sum_{i=1}^{l} \frac{1}{\left|k_{r i}^{*}\right|}\left(\tilde{\boldsymbol{k}}_{x i}^{\top} \boldsymbol{\Gamma}_{i}^{-1} \tilde{\boldsymbol{k}}_{x i}+\gamma_{i}^{-1} \tilde{k}_{r i}^{2}\right)
$$

Suppose that $\chi_{i}(t)=1$ for $t \in\left[t_{k-1}, t_{k}\right)$, then over this time interval the derivative of $V$ along (5.3.5)-(5.3.6) is

$$
\dot{V}=-\boldsymbol{e}^{\top} \boldsymbol{e}+\frac{1}{\left|k_{r i}^{*}\right|}\left(\tilde{\boldsymbol{k}}_{x i}^{\top} \boldsymbol{\Gamma}_{i}^{-1} \boldsymbol{f}_{x i}+\gamma_{i}^{-1} \tilde{k}_{r i} f_{r i}\right)
$$

Here, without loss of generality, we consider the case $\boldsymbol{Q}_{m i}=\boldsymbol{I}_{n}, i \in \mathcal{I}$. It can be verified that $\left(k_{x i j}(t)-k_{x i j}^{*}\right) f_{x i j}(t) \leq 0,\left(k_{r i}(t)-k_{r i}^{*}\right) f_{r i}(t) \leq 0$. Furthermore, with the parameter projection adaptive laws, the parameter estimates $\boldsymbol{k}_{x i}(t), k_{r i}(t)$ are bounded, thus there exists a $c_{p}>0$, defined as

$$
c_{p}=\max _{i \in \mathcal{I}}\left(\frac{1}{\left|k_{r i}^{*}\right|} \tilde{\boldsymbol{k}}_{x i}^{\top} \boldsymbol{\Gamma}_{i}^{-1} \tilde{\boldsymbol{k}}_{x i}+\frac{1}{\left|k_{r i}^{*}\right|} \gamma_{i}^{-1} \tilde{k}_{r i}^{2}\right)
$$

such that

$$
\dot{V} \leq-\frac{V}{(1+\kappa) \alpha}-\frac{\kappa V-(1+\kappa) l c_{p}}{(1+\kappa) \alpha}, \quad \kappa>0
$$


that is, for $V>l c_{p}(1+\kappa) / \kappa, V$ decays faster than exponentially at the rate $-1 /(1+$ $\kappa) \alpha$, and is non-increasing otherwise.

When a subsystem switch occurs at $t=t_{k}$, following the proof of Lemma 5.2.1, we have

$$
V\left(t_{k}\right) \leq\left(1+\mu \Delta_{\boldsymbol{A}_{m}}\right) V\left(t_{k}^{-}\right)
$$

and the slow switching condition $T_{0} \geq T_{d}$ ensures that

$$
V\left(t_{k}\right) \leq\left\{\begin{array}{cl}
l c_{p}\left(1+\mu \Delta_{\boldsymbol{A}_{m}}\right) \frac{1+\kappa}{\kappa}, & V\left(t_{k}^{-}\right) \leq l c_{p} \frac{1+\kappa}{\kappa} \\
V\left(t_{k-1}\right), & V\left(t_{k}^{-}\right)>l c_{p} \frac{1+\kappa}{\kappa}
\end{array}\right.
$$

Therefore, $V(t) \leq c_{0} \triangleq \max \left\{l c_{p}\left(1+\mu \Delta_{\boldsymbol{A}_{m}}\right)(1+\kappa) / \kappa, V\left(t_{0}\right)\right\}$, and closed-loop stability can be concluded.

For evaluating the tracking performance, there are four possible cases depending on the integration interval $[t, t+T]$.

(i) $T \leq T_{0}, t_{k-1} \leq t \leq t+T<t_{k}$. There is no subsystem switch over $[t, t+T]$, and we have

$$
\int_{t}^{t+T} \boldsymbol{e}^{\top}(\tau) \boldsymbol{e}(\tau) d \tau \leq c_{0}
$$

(ii) $T \leq T_{0}, t<t_{k} \leq t+T$. There is one and only one switch at $t=t_{k}$. We have

$$
\dot{V} \leq-\boldsymbol{e}^{\top}(t) \boldsymbol{e}(t)+\boldsymbol{e}^{\top}(t) \Delta \boldsymbol{P}_{m(k)} \delta\left(t-t_{k}\right) \boldsymbol{e}(t)
$$

with $\delta(t)$ being the unit impulse function, and

$$
\begin{aligned}
\int_{t}^{t+T} \boldsymbol{e}^{\top}(\tau) \boldsymbol{e}(\tau) d \tau & \leq V(t)-V(t+T)+\boldsymbol{e}^{\top}\left(t_{k}\right) \Delta \boldsymbol{P}_{m(k)} \boldsymbol{e}\left(t_{k}\right) \\
& \leq\left(1+\mu \Delta_{\boldsymbol{A}_{m}}\right) c_{0}
\end{aligned}
$$

(iii) $T>T_{0}, t<t_{k}, t+T<t_{k+N}$, where $N$ is the largest integer less than or equal to $T / T_{0}$. There are at most $N$ subsystem switches at $t=t_{k}, t_{k+1}, \ldots, t_{k+N-1}$, 
respectively, and

$$
\begin{aligned}
\int_{t}^{t+T} \boldsymbol{e}^{\top}(\tau) \boldsymbol{e}(\tau) d \tau & \leq V(t)-V(t+T)+\sum_{j=0}^{N-1} \boldsymbol{e}^{\top}\left(t_{k+j}\right) \Delta \boldsymbol{P}_{m}^{k+j} \boldsymbol{e}\left(t_{k+j}\right) \\
& \leq \mu \Delta_{\boldsymbol{A}_{m}} c_{0} \frac{T}{T_{0}}+c_{0}
\end{aligned}
$$

(iv) $T>T_{0}, t<t_{k}, t+T \geq t_{k+N}$. There are at most $N+1$ subsystem switches at $t=t_{k}, t_{k+1}, \ldots, t_{k+N}$, respectively, so that

$$
\int_{t}^{t+T} \boldsymbol{e}^{\top}(\tau) \boldsymbol{e}(\tau) d \tau \leq \mu \Delta_{\boldsymbol{A}_{m}} c_{0} \frac{T}{T_{0}}+\left(1+\mu \Delta_{\boldsymbol{A}_{m}}\right) c_{0}
$$

It can be concluded from (i)-(iv) that (5.3.7) is satisfied.

\subsection{Proof of Theorem 5.3.5}

Suppose that $\chi_{i}(t)=1$ for $t \in\left[t_{k-1}, t_{k}\right), i \in \mathcal{I}, k \in \mathbb{Z}^{+}$. Let $\tilde{\boldsymbol{k}}_{x i}(t) \triangleq \boldsymbol{k}_{x i}(t)-\boldsymbol{k}_{x i}^{*}=$ $\left[\tilde{k}_{x i 1}(t), \ldots, \tilde{k}_{x i n}(t)\right]^{\top}, \tilde{k}_{r i}(t) \triangleq k_{r i}(t)-k_{r i}^{*}$, and denote $\boldsymbol{x}(t)=\left[x_{1}(t), \ldots, x_{n}(t)\right]^{\top}$. Then over $\left[t_{k-1}, t_{k}\right)$, the error equation (5.3.5) can be rewritten as

$$
\dot{\boldsymbol{e}}(t)=\boldsymbol{A}_{m i} \boldsymbol{e}(t)-\boldsymbol{F}^{\boldsymbol{\top}}(t) \tilde{\boldsymbol{\theta}}_{i}^{b}(t)
$$

with $\tilde{\boldsymbol{\theta}}_{i}^{b}=-\left[\boldsymbol{b}_{i}^{\boldsymbol{\top}} \tilde{k}_{x i 1}, \boldsymbol{b}_{i}^{\boldsymbol{\top}} \tilde{k}_{x i 2} \ldots, \boldsymbol{b}_{i}^{\boldsymbol{\top}} \tilde{k}_{x i n}, \boldsymbol{b}_{i}^{\boldsymbol{\top}} \tilde{k}_{r i}\right]^{\boldsymbol{\top}}$ and $\boldsymbol{F}^{\boldsymbol{\top}}(t)=\left[x_{1} \boldsymbol{I}_{n}, x_{2} \boldsymbol{I}_{n}, \ldots, x_{n} \boldsymbol{I}_{n}, r \boldsymbol{I}_{n}\right]$. For simplicity of presentation, let $\boldsymbol{\Gamma}_{i}=\operatorname{diag}\left\{\gamma_{i 1}, \ldots, \gamma_{i n}\right\}$, then the adaptive laws in (5.3.8) lead to

$$
\dot{\tilde{\boldsymbol{\theta}}}_{i}^{b}(t)=\overline{\boldsymbol{P}}_{i 1} \boldsymbol{F}(t) \overline{\boldsymbol{P}}_{i 2} \boldsymbol{e}(t)
$$

with $\overline{\boldsymbol{P}}_{i 1}=\operatorname{sign}\left[k_{r i}^{*}\right] \operatorname{diag}\left\{\gamma_{i 1} \boldsymbol{I}_{n}, \gamma_{i 2} \boldsymbol{I}_{n}, \ldots, \gamma_{i n} \boldsymbol{I}_{n}, \gamma_{i} \boldsymbol{I}_{n}\right\}$ and $\overline{\boldsymbol{P}}_{i 2}=\boldsymbol{b}_{i} \boldsymbol{b}_{m i}^{\top} \boldsymbol{P}_{m i}$. The closed-loop system error dynamics over $\left[t_{k-1}, t_{k}\right)$ can be expressed as

$$
\left[\begin{array}{c}
\dot{\boldsymbol{e}} \\
\dot{\tilde{\boldsymbol{\theta}}_{i}^{b}}
\end{array}\right]=\boldsymbol{A}_{0 i}\left[\begin{array}{c}
\boldsymbol{e} \\
\tilde{\boldsymbol{\theta}}_{i}^{b}
\end{array}\right], \quad \boldsymbol{A}_{0 i}=\left[\begin{array}{cc}
\boldsymbol{A}_{m i} & -\boldsymbol{F}^{\boldsymbol{\top}}(t) \\
\overline{\boldsymbol{P}}_{i 1} \boldsymbol{F}(t) \overline{\boldsymbol{P}}_{i 2} & \mathbf{0}
\end{array}\right]
$$


Consider the Lyapunov-like function

$$
\begin{aligned}
V & =\boldsymbol{z}_{0 i}^{\top} \boldsymbol{P}_{0 i} \boldsymbol{z}_{0 i}, \quad \boldsymbol{z}_{0 i}=\left[\boldsymbol{e}^{\top}, \tilde{\boldsymbol{\theta}}_{i}^{b \top}\right]^{\top} \\
\boldsymbol{P}_{0 i} & =\operatorname{diag}\left\{\boldsymbol{P}_{m i}, \frac{1}{\left\|\boldsymbol{b}_{i}\right\|^{2}\left|k_{r i}^{*}\right|} \operatorname{diag}\left\{\frac{1}{\gamma_{i 1}} \boldsymbol{I}_{n}, \ldots, \frac{1}{\gamma_{i n}} \boldsymbol{I}_{n}, \frac{1}{\gamma_{i}} \boldsymbol{I}_{n}\right\}\right\}
\end{aligned}
$$

Along (5.3.8) and (9.4.3), its time derivative is

$$
\dot{V}=\boldsymbol{z}_{0 i}^{\top}\left(\boldsymbol{A}_{0 i}^{\top} \boldsymbol{P}_{0 i}+\boldsymbol{P}_{0 i} \boldsymbol{A}_{0 i}\right) \boldsymbol{z}_{0 i} \leq-\nu_{i} \boldsymbol{z}_{0 i}^{\top} \boldsymbol{C}_{0} \boldsymbol{C}_{0}^{\top} \boldsymbol{z}_{0 i}
$$

with $\nu_{i}=\lambda_{\min }\left[\boldsymbol{Q}_{m i}\right]$ and $\boldsymbol{C}_{0}^{\boldsymbol{\top}}=\left[\boldsymbol{I}_{n}, \mathbf{0}\right]$; that is,

$$
\boldsymbol{A}_{0 i}^{\top} \boldsymbol{P}_{0 i}+\boldsymbol{P}_{0 i} \boldsymbol{A}_{0 i}+\nu_{i} \boldsymbol{C}_{0} \boldsymbol{C}_{0}^{\top} \leq \mathbf{0}
$$

Since $r(t)$ is sufficiently rich of order $n+1$ and $\left(\boldsymbol{A}_{m i}, \boldsymbol{b}_{m i}\right)$ is controllable, it follows that $\boldsymbol{\phi}_{m}(t) \triangleq\left[\boldsymbol{x}_{m}^{\top}(t), r(t)\right]^{\top}$ is P.E. [14], hence $\boldsymbol{\phi}(t) \triangleq\left[\boldsymbol{x}^{\top}(t), r(t)\right]^{\top}$ is P.E. [84], which together with (9.4.5) results in the exponential stability of the closed-loop system [14, Lemma 5.6.3]; that is, for $\boldsymbol{z}_{i}(t)=\left[\boldsymbol{e}^{\boldsymbol{\top}}(t), \tilde{\boldsymbol{\theta}}_{i}^{\top}(t)\right]^{\boldsymbol{\top}}=\left[\boldsymbol{e}^{\boldsymbol{\top}}(t), \tilde{\boldsymbol{k}}_{x i}^{\boldsymbol{\top}}(t), \tilde{k}_{r i}(t)\right]^{\boldsymbol{\top}}$ and $\chi_{i}(t)=1$ over $\left[t_{k-1}, t_{k}\right)$, there exist positive constants $a_{i}$ and $\lambda_{i}$ such that

$$
\left\|\boldsymbol{z}_{i}(t)\right\| \leq a_{i} e^{-\lambda_{i}\left(t-t_{k-1}\right)}\left\|\boldsymbol{z}_{i}\left(t_{k-1}\right)\right\|, \quad t \in\left[t_{k-1}, t_{k}\right)
$$

Consider the Lyapunov-like function in (9.3.1). It follows with

$$
\alpha_{i}=\lambda_{\max }\left[\boldsymbol{P}_{m i}, \Gamma_{i}^{-1}, \gamma_{i}^{-1}\right], \quad \beta_{i}=\lambda_{\min }\left[\boldsymbol{P}_{m i}, \Gamma_{i}^{-1}, \gamma_{i}^{-1}\right]
$$

and for $\chi_{i}(t)=1$ over $\left[t_{k-1}, t_{k}\right)$ that

$$
\begin{aligned}
V\left(t_{k}^{-}\right)-B_{(k-1)} & \leq \alpha_{i}\left\|\boldsymbol{z}_{i 0}\left(t_{k}^{-}\right)\right\|^{2} \\
& \leq \alpha_{i} a_{i}^{2} e^{-2 \lambda_{i}\left(t-t_{k-1}\right)}\left\|\boldsymbol{z}_{i 0}\left(t_{k-1}\right)\right\|^{2} \\
& \leq \frac{\alpha_{i}}{\beta_{i}} a_{i}^{2} e^{-2 \lambda_{i}\left(t-t_{k-1}\right)}\left(V\left(t_{k-1}\right)-B_{(k-1)}\right)
\end{aligned}
$$

where $B_{(k-1)}=\sum_{j \in \mathcal{I}, j \neq i} \frac{1}{\left|k_{r j}^{*}\right|}\left(\tilde{\boldsymbol{k}}_{x j}^{\top} \boldsymbol{\Gamma}_{j}^{-1} \tilde{\boldsymbol{k}}_{x j}+\gamma_{j}^{-1} \tilde{k}_{r j}^{2}\right)$ is constant over $\left[t_{k-1}, t_{k}\right)$, since $\chi_{j}(t)=0$. Following the proof in Lemma 5.2.1, when a subsystem switch occurs at 
$t=t_{k}$, we have $V\left(t_{k}\right)-V\left(t_{k}^{-}\right) \leq \mu \Delta_{\boldsymbol{A}_{m}}\left(V\left(t_{k}^{-}\right)-B_{(k-1)}\right) ;$ that is,

$$
V\left(t_{k}\right)-B_{(k-1)} \leq\left(1+\mu \Delta_{\boldsymbol{A}_{m}}\right)\left(V\left(t_{k}^{-}\right)-B_{(k-1)}\right)
$$

If $t_{k}-t_{k-1}>\ln \left[\left(\alpha_{i} / \beta_{i}\right) a_{i}^{2}\left(1+\mu \Delta_{\boldsymbol{A}_{m}}\right)\right] / 2 \lambda_{i}$, then $V\left(t_{k}\right)<V\left(t_{k-1}\right)$. Therefore, for $T_{0}=\min _{k \in \mathbb{Z}^{+}}\left\{t_{k}-t_{k-1}\right\}>T_{d}=\max \left\{\alpha \ln \left(1+\mu \Delta_{\boldsymbol{A}_{m}}\right), \ln \left[(\bar{\alpha} / \bar{\beta}) a^{2}\left(1+\mu \Delta_{\boldsymbol{A}_{m}}\right)\right] / 2 \lambda_{i}\right\}$ with $\bar{\alpha}=\max _{i \in \mathcal{I}} \alpha_{i}, \bar{\beta}=\min _{i \in \mathcal{I}} \beta_{i}$, and $a_{s}=\max _{i \in \mathcal{I}} a_{i}$, we have stability of the reference model system. Furthermore, the values of the Lyapunov function at the switching time instants form a strictly decreasing sequence.

If some of the subsystems with index $i \in \mathcal{I}^{*} \subset \mathcal{I}$ are no longer active after a finite time $T_{i} \geq t_{0}$, then

$$
V(\infty)=\sum_{i \in \mathcal{I}^{*}} \frac{1}{\left|k_{r i}^{*}\right|}\left(\tilde{\boldsymbol{k}}_{x i}^{\top}\left(T_{i}\right) \boldsymbol{\Gamma}_{i}^{-1} \tilde{\boldsymbol{k}}_{x i}\left(T_{i}\right)+\gamma_{i}^{-1} \tilde{k}_{r i}^{2}\left(T_{i}\right)\right)
$$

that is, $\boldsymbol{e}(t), \tilde{\boldsymbol{k}}_{x i}(t)$, and $\tilde{k}_{r i}(t)$ converge to zero asymptotically for $i \notin \mathcal{I}^{*}$. On the other hand, $\boldsymbol{k}_{x i}(t)=\boldsymbol{k}_{x i}\left(T_{i}\right), k_{r i}(t)=k_{r i}\left(T_{i}\right), i \in \mathcal{I}^{*}, t \geq T_{i}$, due to the fact that $\chi_{i}(t)=0$.

In addition, with $T_{d}^{\prime} \triangleq \sup _{i \in \mathcal{I}}\left\{\ln a_{i} / \lambda_{i}\right\}, \lambda=\inf _{i \in \mathcal{I}}\left\{\lambda_{i}-\ln a_{i} / T_{d}^{\prime}\right\}$, it follows that $\lambda \tau \leq \lambda_{i} \tau-\ln a_{i}$, i.e., $a_{i} e^{-\lambda_{i} \tau} \leq e^{-\lambda \tau}, \tau \geq T_{d}^{\prime}$; on the other hand, we have $a_{i} e^{-\lambda_{i} \tau} \leq a_{s} e^{-\lambda \tau}, \tau<T_{d}^{\prime}$. Suppose $T^{*}=t_{j-1}$ for some $j \in \mathbb{Z}^{+}$, then with the fact that $\|\boldsymbol{e}(t)\|,\left\|\tilde{\boldsymbol{\theta}}_{i}(t)\right\| \leq\left\|\boldsymbol{z}_{i}(t)\right\| \leq\|\boldsymbol{e}(t)\|+\left\|\tilde{\boldsymbol{\theta}}_{i}(t)\right\|, i \in \mathcal{I}-\mathcal{I}^{*}$, we have for $t \in\left[t_{k-1}, t_{k}\right)$ and $T_{0} \geq T_{d}$ that

$$
\begin{aligned}
\left\|\boldsymbol{z}_{i_{(k)}}(t)\right\| \leq & a_{s} e^{-\lambda\left(t-t_{k-1}\right)}\left\|\boldsymbol{z}_{i_{(k)}}\left(t_{k-1}\right)\right\| \\
\leq & a_{s} e^{-\lambda\left(t-t_{k-2}\right)}\left\|\boldsymbol{z}_{i_{(k-1)}}\left(t_{k-2}\right)\right\|+a_{s} e^{-\lambda\left(t-t_{k-1}\right)}\left\|\boldsymbol{z}_{i_{(k)}}\left(t_{k-2}\right)\right\| \\
& \vdots \\
\leq & a_{s} F(k-j+1) e^{-\frac{\lambda}{N}\left(t-t_{j-1}\right)}\left\|\boldsymbol{z}_{i_{(j)}}\left(t_{j-1}\right)\right\| \\
& +\sum_{i} a_{s} F(k-j) e^{-\frac{\lambda}{N}\left(t-t_{j-1}\right)} e^{\lambda\left(t_{j}-t_{j-1}\right)}\left\|\boldsymbol{z}_{i}\left(t_{j-1}\right)\right\|
\end{aligned}
$$


where $i_{(\cdot)} \in \mathcal{I}-\mathcal{I}^{*}, i \in \mathcal{I}-\mathcal{I}^{*}$ but $i \neq i_{(j)}$ in the summation, $N$ is the number of different subsystems in $\mathcal{I}-\mathcal{I}^{*}$, and $F(m)$ denotes the sum of the first $m$ Fibonacci numbers, i.e.,

$$
F(m)=\frac{1}{\sqrt{5}}\left[\psi^{m}-(1-\psi)^{m}\right], \quad \psi=\frac{1+\sqrt{5}}{2}
$$

Furthermore, we have

$$
\begin{aligned}
\psi^{k-j+1} e^{-\frac{\lambda}{N}\left(t-t_{j-1}\right)} & \leq e^{\frac{\lambda}{4 N} T_{0}} e^{-\frac{\lambda}{2 N}\left(t-t_{j-1}\right)} \\
\psi^{k-j} e^{-\frac{\lambda}{N}\left(t-t_{j-1}\right)} & \leq e^{-\frac{\lambda}{2 N}\left(t-t_{j-1}\right)}
\end{aligned}
$$

if $T_{0}>2 N \ln \psi / \lambda$, and

$$
-(1-\psi)^{k-j+1} e^{-\frac{\lambda}{N}\left(t-t_{j-1}\right)} \leq e^{-\frac{\lambda}{2 N}\left(t-t_{j-1}\right)}
$$

(similarly for $\left.-(1-\psi)^{k-j}\right)$. Therefore, with the minimum switching time interval $T_{0}>\max \left\{T_{d}, T_{d}^{\prime}, \frac{2 N \ln \psi}{\lambda}\right\}$, we have

$$
\left\|\boldsymbol{z}_{i(k)}(t)\right\| \leq \sum_{i \in \mathcal{I}-\mathcal{I}^{*}} c_{i} e^{-\frac{\lambda}{2 N}\left(t-T^{*}\right)}\left\|\boldsymbol{z}_{i}\left(T^{*}\right)\right\|, \quad t \geq T^{*}
$$

for $c_{i(k)}=\frac{a_{s}}{\sqrt{5}}\left(e^{\frac{\lambda}{4 N} T_{0}}-1+\psi\right), c_{i}=\frac{a_{s}}{\sqrt{5}} e^{\lambda\left(t_{j}-t_{j-1}\right)} \psi, i \in \mathcal{I}-\mathcal{I}^{*}, i \neq i_{(k)}$, and $\boldsymbol{e}(t), \tilde{\boldsymbol{k}}_{x i}(t)$, and $\tilde{k}_{r i}(t)$ converge to zero exponentially, $i \in \mathcal{I}-\mathcal{I}^{*}, t \geq T^{*}$. 


\section{Bibliography}

[1] D. Liberzon, Switching in Systems and Control. Boston, MA: Birkhäuser, 2003.

[2] Z. Sun and S. S. Ge, Switched Linear Systems: Control and Design. London: Springer-Verlag, 2005.

[3] W. P. Dayawansa and C. F. Martin, "A converse Lyapunov theorem for a class of dynamical systems which undergo switching," IEEE Transactions on Automatic Control, vol. 44, pp. 751-760, 1999.

[4] R. W. Brockett, "Hybrid models for motion control systems," in Essays on Control: Perspectives in the Theory and Its Applications, H. Trentelman and J. C. Willems, Eds. Boston: Birkhäuser, 1993, pp. 29-53.

[5] H. D. Jong, C. H. J. Gouzé, M. Page, T. Sari, and J. Geiselmann, "Qualitative simulation of genetic regulatory networks using piecewise-linear models," Bulletin of Mathematical Biology, vol. 66, pp. 301-340, 2004.

[6] F. Tahami, M. Mobed, and B. Molayee, "On piecewise affine large-signal modeling of PWM converters," in Proceedings of the 2006 IEEE International Conference on Industrial Technology, Bombay, India, Dec. 2006, pp. 1419-1423.

[7] G. F. Franklin, J. D. Powell, and A. Emami-Naeini, Automatic Control Systems, 5th ed. Upper Saddle River, NJ: Pearson Prentice Hall, 2006.

[8] B. C. Kuo and F. Golnaraghi, Automatic Control Systems, 8th ed. New York: John Wiley and Sons, 2003. 
[9] K. S. Narendra and A. M. Annaswamy, Stable Adaptive Systems. Englewood Cliffs, NJ: Prentice-Hall, 1989.

[10] S. Sastry and M. Bosdon, Adaptive Control: Stability, Convergence and Robustness. $\quad$ Englewood Cliffs, NJ: Prentice-Hall, 1989.

[11] K. S. Tsakalis and P. A. Ioannou, Linear Time-Varying Systems: Control and Adaptation. Englewood Cliffs, NJ: Prentice-Hall, 1993.

[12] K. J. Åström and B. Wittenmark, Adaptive Control, 2nd ed. Boston, MA, USA: Addison-Wesley Longman Publishing Co., Inc., 1994.

[13] M. Krstic, P. V. Kokotovic, and I. Kanellakopoulos, Nonlinear and Adaptive Control Design, 1st ed. New York, NY, USA: John Wiley \& Sons, Inc., 1995.

[14] P. Ioannou and J. Sun, Robust Adaptive Control. Prentice Hall, 1996.

[15] G. Tao, Adaptive Control Design and Analysis. New York: Wiley, 2003.

[16] A. Annaswamy, J. Jang, and E. Lavretsky, "Stability margins for adaptive controllers in the presence of time-delay," in Proceedings of 2008 AIAA Guidance, Navigation and Control Conference, no. AIAA-2008-6659, Honolulu, Hawaii, Aug. 2008.

[17] N. T. Nguyen, "Verifiable adaptive control with analytical stability margins by optimal control modification," in Proceedings of 2010 AIAA Guidance, Navigation and Control Conference, Toronto, Ontario, Canada, Aug. 2010.

[18] C. Cao and N. Hovakimyan, "Stability margins of $l_{1}$ adaptive control architecture," IEEE Transactions on Automatic Control, vol. 55, no. 2, pp. 480-487, Feb. 2010.

[19] D. Liberzon and A. S. Morse, "Basic problems in stability and design of switched systems," IEEE Control Systems Magazine, vol. 19, pp. 59-70, Oct. 1999. 
[20] A. N. Michel, "Recent trends in the stability analysis of hybrid dynamical systems," IEEE Transactions on Circuits and Systems I: Fundamental Theory and Applications, vol. 46, no. 1, pp. 120-134, Jan. 1999.

[21] R. A. Decarlo, M. S. Branicky, S. Pettersson, and B. Lennartson, "Perspectives and results on the stability and stabilizability of hybrid systems," Proceedings of the IEEE, vol. 88, no. 7, pp. 1069-1082, Jul 2000.

[22] Z. Sun and S. S. Ge, "Analysis and synthesis of switched linear control systems," Automatica, vol. 41, no. 2, pp. 181-195, 2005.

[23] H. Lin and P. J. Antsaklis, "Stability and stabilizability of switched linear systems: a short survey of recent results," in Proceedings of the 2005 IEEE International Symposium on Intelligent Control and Mediterrean Conference on Control and Automation, Jun. 2005, pp. 24-29.

[24] — - "Stability and stabilizability of switched linear systems: a survey of recent results," IEEE Transactions on Automatic Control, vol. 54, no. 2, pp. 308-322, Feb. 2009.

[25] P. J. Antsaklis and A. Nerode, "Hybrid control systems: An introductory discussion to the special issue," IEEE Transactions on Automatic Control, vol. 43, no. 4, pp. 457-460, Apr 1998.

[26] A. S. Morse, C. Pantelides, S. Sastry, and J. M. Schumacher, Eds., Automatica, Mar. 1999, vol. 35, no. 3, Special Issue on Hybrid Systems.

[27] P. J. Antsaklis, Ed., Proceedings of the IEEE, Jul. 2000, vol. 88, no. 7, Special Issue on Hybrid Systems: Theory and Applications.

[28] M. di. Bernardo, H. S. Chung, and C. K. Tse, Eds., IEEE Transactions on Circuits and Systems, I: Fundamental Theory and Applications, Aug. 2003, vol. 50, no. 8, Special Issue on Switching and Systems. 
[29] P. Peleties and R. DeCarlo, "Asymptotic stability of $m$-switched systems using Lyapunov-like functions," in Proceedings of the 1991 American Control Conference, Boston, MA, Jun. 1991, pp. 3438-3439.

[30] K. S. Narendra and J. Balakrishnan, "A common Lyapunov function for stable LTI systems with commuting A-matrices," IEEE Transactions on Automatic Control, vol. 39, pp. 2469-2471, Dec. 1994.

[31] Y. Mori, T. Mori, and Y. Kuroe, "A solution to the common Lyapunov function problem for continuous-time systems," in Proceedings of the 36th Conference on Decision and Control, San Diego, California, Dec. 1997, pp. 3530-3531.

[32] T. Ooba and Y. Funahashi, "On a common quadratic Lyapunov function for widely distant systems," IEEE Transactions on Automatic Control, vol. 42, pp. 1697-1699, Dec. 1997.

[33] R. Shorten and K. S. Narendra, "On the stability and existence of common Lyapunov functions for stable linear switching systems," in Proceedings of the 37th IEEE Conference on Decision and Control, Tampa, FL, Dec. 1998, pp. 3723-3724.

[34] H. Shim, D. J. Noh, and J. H. Seo, "Common Lyapunov function for exponentially stable nonlinear systems," in Proceedings of the 4 th SIAM Conference on Control and Its Applications, Jaxsonville, FL, May. 1998, pp. 1410-1414.

[35] R. Shorten and K. S. Narendra, "Necessary and sufficient conditions for the existence of a common quadratic Lyapunov function for two stable second-order linear time-invariant systems," in Proceedings of the 1999 American Control Conference, San Diego, CA, Jun. 1999, pp. 1410-1414.

[36] R. Shorten, K. S. Narendra, and O. Mason, "A result on common quadratic Lyapunov functions," IEEE Transactions on Automatic Control, vol. 48, no. 1, pp. 110-113, Jan. 2003. 
[37] L. Vu and D. Liberzon, "Common Lyapunov functions for families of commuting nonlinear systems," Systems \& Control Letters, vol. 54, no. 5, pp. 405-416, 2005.

[38] A. S. Morse, "Supervisory control of families of linear set-point controllers-part 1: exact matching," IEEE Transactions on Automatic Control, vol. 41, pp. 14131431, Oct. 1996.

[39] J. P. Hespanha and A. S. Morse, "Stability of switched systems with average dwell-time," EE-Systems, University of Southern California/Lab. for Control Science \& Eng., Yale University, Santa Barbara, Tech. Rep., Jan. 1999.

[40] — - "Stability of switched systems with average dwell-time," in Proceedings of the 38th IEEE Conference on Decision and Control, Dec. 1999, pp. 2655-2660.

[41] G. Zhai, B. Hu, K. Yasuda, and A. N. Michel, "Stability analysis of switched systems with stable and unstable subsystems: an average dwell time approach," International Journal of Systems Science, vol. 32, pp. 1055-1061, 2001.

[42] M. A. Müller and D. Liberzon, "Input/outpu-to-state stability of switched nonlinear systems," in Proceedings of the 2010 American Control Conference, Baltimore, MD, Jul. 2010, pp. 1708-1712.

[43] M. A. Müller and F. Allgöwer, "Model predictive control of switched nonlinear systems under average dwell-time," in Proceedings of the 2011 American Control Conference, San Francisco, CA, Jul. 2011, pp. 5169-5174.

[44] — _ "Improving performance in model predictive control: switching cost functionals under average dwell-time," Automatica, vol. 48, pp. 402-409, 2012.

[45] M. A. Wicks, P. Peleties, and R. A. DeCarlo, "Construction of piecewise Lyapunov functions for stabilizing switched systems," in Proceedings of the 33th IEEE Conference on Decision and Control, Dec. 1994, pp. 3492-3497.

[46] S. Pettersson and B. Lennartson, "LMI for stability and robustness of hybrid systems," in Proceedings of the 1997 American Control Conference, Jun. 1997, pp. $1714-1718$. 
[47] M. S. Branicky, "Multiple Lyapunov functions and other analysis tools for switched and hybrid systems," IEEE Transactions on Automatic Control, vol. 43, no. 4, pp. 475-482, Apr. 1998.

[48] A. N. Michel and B. Hu, "Towards a stability theory of general hybrid dynamical systems," Automatica, vol. 35, pp. 371-384, 1998.

[49] M. A. Wicks, P. Peleties, and R. A. Decarlo, "Switched controller synthesis for the quadratic stabilization of a pair of unstable linear systems," European Journal of Control, vol. 4, pp. 140-147, 1998.

[50] H. Ye, A. N. Michel, and L. Hou, "Stability theory for hybrid dynamical systems," IEEE Transactions on Automatic Control, vol. 43, no. 4, pp. 461-474, Apr. 1998.

[51] B. Hu, X. Xu, A. N. Michel, and P. J. Antsaklis, "Stability analysis for a class of nonlinear switched systems," in Proceedings of the 38th IEEE Conference on Decision and Control, Dec. 1999, pp. 4374-4379.

[52] S. Pettersson and B. Lennartson, "Exponential stability of hybrid systems using piecewise quadratic Lyapunov functions resulting in LMIs," in Proceedings of the 14th Triennial World Congress of the IFAC, 1999.

[53] A. Agrachev and D. Liberzon, "Lie-algebraic stability criteria for switched systems," SIAM Journal of Control and Optimization, vol. 40, pp. 253-269, Jan. 2001.

[54] D. Cheng, L. Guo, and J. Huang, "On quadratic Lyapunov functions," IEEE Transactions on Automatic Control, vol. 48, no. 5, pp. 885-890, May. 2003.

[55] S. Ching and E. J. Davison, "A switching approach to the control of jump parameter systems," in Proceedings of the 2006 American Control Conference, Minneapolis, Minnesota, Jun. 2006, pp. 5419-5424.

[56] K. S. Narendra and J. Balakrishnan, "Adaptive control using multiple models," IEEE Transactions on Automatic Control, vol. 42, pp. 171-187, 1997. 
[57] K. S. Tsakalis, "Model reference adaptive control of linear time-varying plants: the case of "jump' parameter variations," International Journal of Control, vol. 56, pp. 1299-1345, 1992.

[58] M. di Bernardo, U. Montanaro, and S. Santini, "Novel switched model reference adaptive control for continuous piecewise affine systems," in the 4 "th IEEE Conference on Decision and Control, Dec. 2008, pp. 1925-1930.

[59] — - "Minimal control synthesis asdaptive control of continuous bimodal piecewise affine systems," SIAM Journal on Control and Optimization, vol. 48, no. 7, pp. 4242-4261, 2010.

[60] W. O. Dennis and G. Tao, "Adaptive control of plants with jumping parameters," in Proceedings of the Third SIAM Conference on Control and Its Applications, St. Louis, MO, Apr. 1995.

[61] Q. Sang and G. Tao, "Gain margins of model reference adaptive control systems," in Proceedings of the 7th World Congress on Intelligent Control and Automation, Chongqing, China, Jun. 2008, pp. 2946-2951.

[62] — - "Gain margins of multivariable MRAC systems," in Proceedings of the

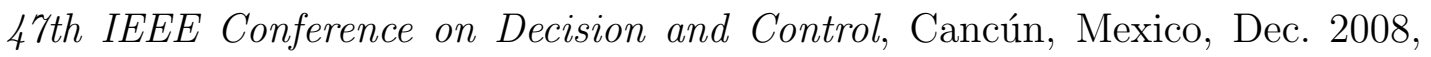
pp. $702-707$.

[63] — _ "Gain margins of adaptive control systems," IEEE Transactions on Automatic Control, vol. 55, no. 1, pp. 104-115, Jan. 2010.

[64] — - "Performance robustness of MRAC under reduction in actuator effectiveness," in Proceedings of the 2009 American Control Conference, St. Louis, MO, Jun. 2009, pp. 4506-4511.

[65] — - "Adaptive control of piecewise linear systems: the state tracking case," in Proceedings of the 2010 American Control Conference, Baltimore, MD, Jun. 2010, pp. 4040-4045. 
[66] — - "Adaptive control of piecewise linear systems with applications to NASA GTM," in Proceedings of the 2011 American Control Conference, San Francisco, CA, Jun. 2011, pp. 1157-1162.

[67] _ - "Adaptive control of piecewise linear systems: the state tracking case," IEEE Transactions on Automatic Control, vol. 57, no. 2, pp. 522-528, Feb. 2012.

[68] — _ "Multivariable adaptive control of piecewise linear systems with applications to NASA Generic Transport Model," accepted for publication in AIAA Journal of Guidance, Control, and Dynamics.

[69] — - "Adaptive control of piecewise linear systems: the output tracking case," in Proceedings of the 2011 American Control Conference, San Francisco, CA, Jun. 2011, pp. 2000-2005.

[70] — - "Multivariable state feedback for output tracking MRAC for piecewise linear systems," in Proceedings of the 50th IEEE Conference on Decision and Control and European Control Conference, Orlando, FL, Dec. 2011, pp. 17841789.

[71] — , "Adaptive control of piecewise linear systems with state feedback for output tracking," submitted to Asian Journal of Control.

[72] — - "Adaptive control of piecewise linear systems with output feedback for output tracking," submitted to the 51st IEEE Conference on Decision and Control.

[73] A. K. Imai, R. R. Costa, L. Hsu, G. Tao, and P. V. Kokotovic, "Multivariable adaptive control using high-frequency gain matrix factorization," IEEE Transactions on Automatic Control, vol. 49, no. 7, pp. 1152-1156, Jul. 2004.

[74] A. Isidori, Nonlinear Control Systems, 3rd ed. Berlin: Springer-Verlag, 1995.

[75] J. X. Guo, Y. Liu, and G. Tao, "Multivariable MRAC with state feedback for output tracking," in Proceedings of the 2009 American Control Conference, St. Louis, MO, Jun. 2009, pp. 592-597. 
[76] M. D. Mathelin and M. Bodson, "Multivariable model reference adaptive control with unknown high frequency gain," in Proceedings of the 32nd IEEE Conference on Decision and Control, San Antonio, TX, Dec. 1993, pp. 3078-3083.

[77] G. C. Goodwin and K. S. Sin, Adaptive Filtering Prediction and Control, 3rd ed. Englewood Cliffs, NJ,: Prentice-Hall, 1984.

[78] H. Elliott and W. A. Wolovich, "Parametrization issues in multivariable adaptive control," Automatica, vol. AC-20, pp. 533-545, 1984.

[79] K. J. Åström, P. H. Hagander, and J. Sternby, "Zeros of sampled sysems," $A u$ tomatica, vol. 20, pp. 31-38, 1983.

[80] S. Hara, H. Katori, and R. Kondo, "The relationship between real poles and real zeros in SISO sampled data systems," IEEE Transactions on Automatic Control, vol. 34, pp. 632-635, 1989.

[81] M. Ishitobi, "A stability condition of zeros of sampled multivariable systems," IEEE Transactions on Automatic Control, vol. 45, pp. 295-299, 2000.

[82] A. M. Murch, "A flight control system architecture for the NASA AirSTAR flight test infrastructure," in Proceedings of 2008 AIAA Guidance, Navigation and Control Conference, no. AIAA-2008-7285, Honolulu, Hawaii, Aug. 2008.

[83] K. S. Tsakalis and P. A. Ioannou, "Adaptive control of linear time-varying plants: a new model reference controller structure," IEEE Transactions on Automatic Control, vol. 34, pp. 1038-1046, 1989.

[84] S. Boyd and S. Sastry, "On parameter convergence in adaptive control," Systems 6 Control Letters, vol. 3, pp. 311-319, 1983. 